Supporting Information

for

\title{
A General Descriptor $\triangle E$ Enables the Quantitative Development of Luminescent Materials based on Photoinduced Electron Transfer
}

\begin{abstract}
Weijie Chi, ${ }^{+, \neq}$Jie Chen, ${ }^{\neq, \neq}$Wenjuan Liu, ${ }^{\ddagger, \neq}$ Chao Wang, ${ }^{+, \neq}$Qingkai Qi, ${ }^{\ddagger}$ Qinglong Qiao, ${ }^{\ddagger}$ Tee Meng Tan, ${ }^{+}$ Kangming Xiong, ${ }^{\ddagger}$ Xiao Liu, ${ }^{\S}$ Keegan Kang, ${ }^{+}$Young-Tae Chang, ${ }^{,{ }^{*}}$ Zhaochao Xu, ${ }^{,,}$Xiaogang Liu ${ }^{+, *}$
\end{abstract}

${ }^{\dagger}$ Fluorescence Research Group, Singapore University of Technology and Design, 8 Somapah Road 487372, Singapore

${ }^{\ddagger}$ CAS Key Laboratory of Separation Science for Analytical Chemistry, Dalian Institute of Chemical Physics, Chinese Academy of Sciences 457 Zhongshan Road, Dalian 116023, China

${ }^{\S}$ Department of Chemistry, POSTECH \& Center for Self-assembly and Complexity, IBS, Pohang 37673, Republic of Korea 


\section{Table of Contents}

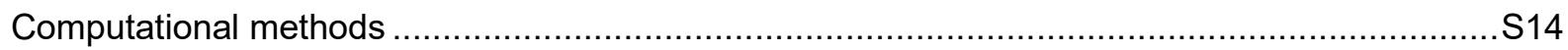

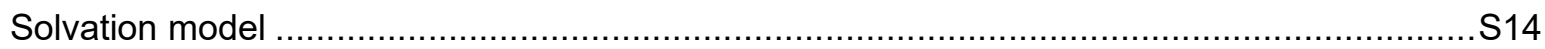

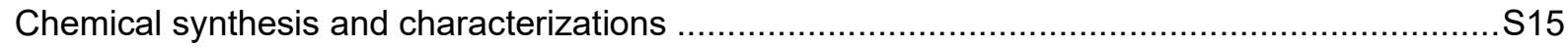

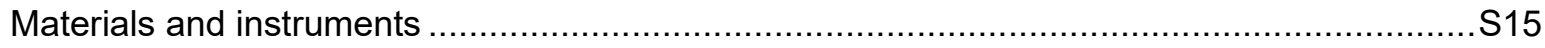

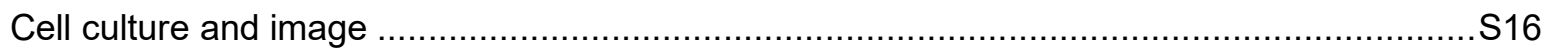

Chemical synthesis of BODIPY derivatives .................................................................. 16

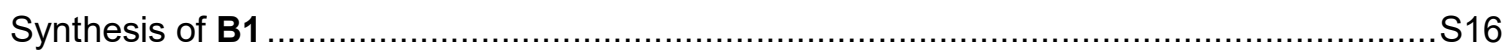

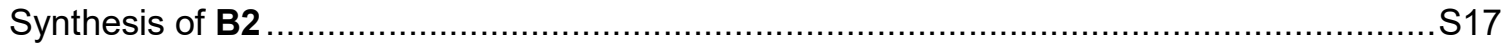

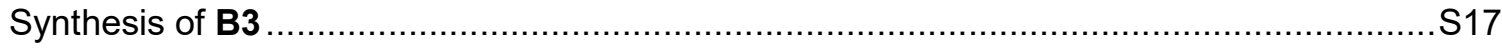

Synthesis of B4

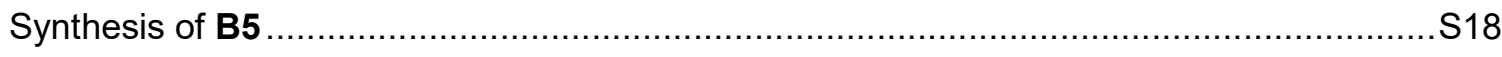

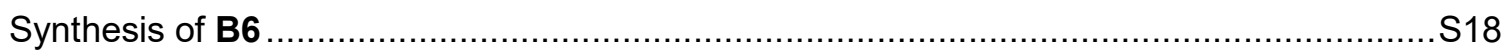

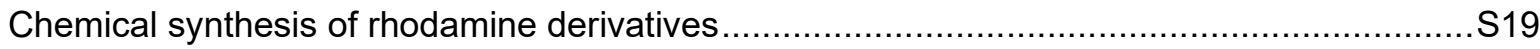

Synthesis of M1

Synthesis of M3

Synthesis of M2

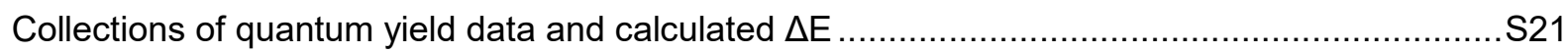

Calculated frontier molecular orbitals and corresponding $\triangle \mathrm{E}$ of meso-phenyl substituted BODIPY-

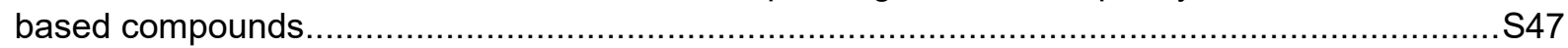

${ }^{1} \mathrm{H}-\mathrm{NMR}$ spectra of B1-B6 and ${ }^{1} \mathrm{H}-\mathrm{NMR},{ }^{13} \mathrm{C}-\mathrm{NMR}$, and HRMS of M1-M3............................S90

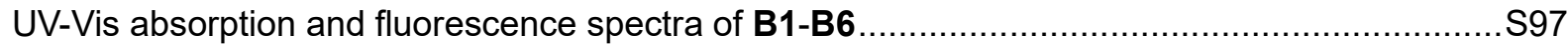

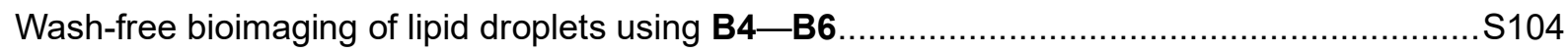

Viscosity dependence of the UV-Vis absorption and emission spectra of B4-B6 ..................S105

Calculated frontier molecular orbitals and corresponding $\Delta E$ of meso-phenyl substituted fluorescein

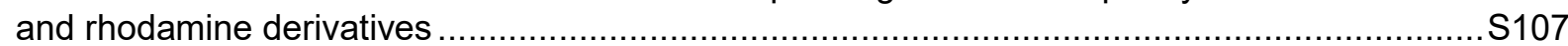

UV-Vis absorption and fluorescence spectra of M1-M3 .................................................. 138

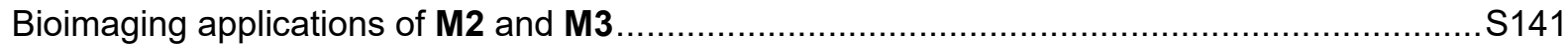

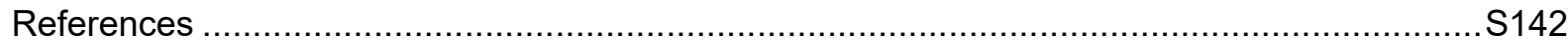




\section{LIST OF FIGURES}

Figure S1. (a) Molecular structure of p-NH2-Ph-BDP (B4) and its quantum yield in ethanol; (b) optimized geometry of B4 in the ground state, frontier molecular orbitals and corresponding energy levels in the ground state in ethanol at M062X/Def2SVP (c) and wB97XD/Def2SVP (d) levels; $f$ refers to the oscillator strength in the first excited state; $98 \%$ of contribution is from HOMO to LUMO transition. Importantly, the energy level of quenching orbital (represented by a blue line) is

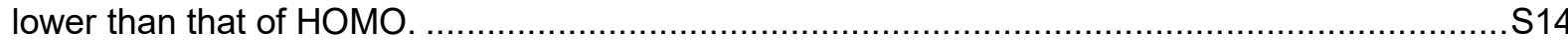
Figure S2. Calculated $\triangle \mathrm{E}$ using PCM and SMD solvation models at M062X/Def2SVP level......S15 Figure S3. a) Molecular structure, b) optimized structure, c) frontier molecular orbitals and corresponding energy levels, and calculated $\triangle \mathrm{E}$ of $\mathrm{B} 1$ in the ground state in ethanol at M062X/Def2SVP level.

Figure S4. a) Molecular structure, b) optimized structure, c) frontier molecular orbitals and corresponding energy levels, and calculated $\triangle \mathrm{E}$ of $\mathbf{B 2}$ in the ground state in ethanol at M062X/Def2SVP level.

Figure S5. a) Molecular structure, b) optimized structure, c) frontier molecular orbitals and corresponding energy levels, and calculated $\triangle \mathrm{E}$ of $\mathrm{B} 3$ in the ground state in methanol at M062X/Def2SVP level.

Figure S6. a) Molecular structure, b) optimized structure, c) frontier molecular orbitals and corresponding energy levels, and calculated $\triangle \mathrm{E}$ of $\mathrm{B} 4$ in the ground state in methanol at M062X/Def2SVP level.

Figure S7. a) Molecular structure, b) optimized structure, c) frontier molecular orbitals and corresponding energy levels, and calculated $\triangle \mathrm{E}$ of $\mathrm{B} 5$ in the ground state in water at M062X/Def2SVP level.

Figure S8. a) Molecular structure, b) optimized structure, c) frontier molecular orbitals and corresponding energy levels, and calculated $\Delta \mathrm{E}$ of $\mathbf{B 6}$ in the ground state in water at M062X/Def2SVP level.

Figure S9. a) Molecular structure, b) optimized structure, c) frontier molecular orbitals and corresponding energy levels, and calculated $\triangle \mathrm{E}$ of $\mathbf{B} 7$ in the ground state in water at M062X/Def2SVP level.

Figure S10. a) Molecular structure, b) optimized structure, c) frontier molecular orbitals and corresponding energy levels, and calculated $\triangle \mathrm{E}$ of $\mathbf{B 8}$ in the ground state in water at M062X/Def2SVP level.

Figure S11. a) Molecular structure, b) optimized structure, c) frontier molecular orbitals and corresponding energy levels, and calculated $\triangle \mathrm{E}$ of $\mathbf{B 9}$ in the ground state in water at M062X/Def2SVP level.

Figure S12. a) Molecular structure, b) optimized structure, c) frontier molecular orbitals and corresponding energy levels, and calculated $\triangle \mathrm{E}$ of B10 in the ground state in water at M062X/Def2SVP level.

Figure S13. a) Molecular structure, b) optimized structure, c) frontier molecular orbitals and corresponding energy levels, and calculated $\triangle \mathrm{E}$ of $\mathrm{B} 11$ in the ground state in water at M062X/Def2SVP level.

Figure S14. a) Molecular structure, b) optimized structure, c) frontier molecular orbitals and corresponding energy levels, and calculated $\triangle \mathrm{E}$ of B12 in the ground state in water at M062X/Def2SVP level.

Figure S15. a) Molecular structure, b) optimized structure, c) frontier molecular orbitals and corresponding energy levels, and calculated $\triangle \mathrm{E}$ of B13 in the ground state in water at M062X/Def2SVP level.

Figure S16. a) Molecular structure, b) optimized structure, c) frontier molecular orbitals and corresponding energy levels, and calculated $\triangle \mathrm{E}$ of B14 in the ground state in water at M062X/Def2SVP level. 
Figure S17. a) Molecular structure, b) optimized structure, c) frontier molecular orbitals and corresponding energy levels, and calculated $\triangle \mathrm{E}$ of B15 in the ground state in water at M062X/Def2SVP level.

Figure S18. a) Molecular structure, b) optimized structure, c) frontier molecular orbitals and corresponding energy levels, and calculated $\triangle \mathrm{E}$ of B16 in the ground state in ethanol at M062X/Def2SVP level.

Figure S19. a) Molecular structure, b) optimized structure, c) frontier molecular orbitals and corresponding energy levels, and calculated $\Delta \mathrm{E}$ of B17 in the ground state in methanol at M062X/Def2SVP level.

Figure S20. a) Molecular structure, b) optimized structure, c) frontier molecular orbitals and corresponding energy levels, and calculated $\triangle \mathrm{E}$ of $\mathrm{B} 18$ in the ground state in methanol at M062X/Def2SVP level.

Figure S21. a) Molecular structure, b) optimized structure, c) frontier molecular orbitals and corresponding energy levels, and calculated $\triangle \mathrm{E}$ of $\mathrm{B} 19$ in the ground state in methanol at M062X/Def2SVP level.

Figure S22. a) Molecular structure, b) optimized structure, c) frontier molecular orbitals and corresponding energy levels, and calculated $\triangle \mathrm{E}$ of B20 in the ground state in methanol at M062X/Def2SVP level......

Figure S23. a) Molecular structure, b) optimized structure, c) frontier molecular orbitals and corresponding energy levels, and calculated $\triangle E$ of B21 in the ground state in methanol at M062X/Def2SVP level.

Figure S24. a) Molecular structure, b) optimized structure, c) frontier molecular orbitals and corresponding energy levels, and calculated $\triangle \mathrm{E}$ of B22 in the ground state in methanol at M062X/Def2SVP level.

Figure S25. a) Molecular structure, b) optimized structure, c) frontier molecular orbitals and corresponding energy levels, and calculated $\triangle \mathrm{E}$ of B23 in the ground state in methanol at M062X/Def2SVP level.

Figure S26. a) Molecular structure, b) optimized structure, c) frontier molecular orbitals and corresponding energy levels, and calculated $\triangle \mathrm{E}$ of B24 in the ground state in methanol at M062X/Def2SVP level.

Figure S27. a) Molecular structure, b) optimized structure, c) frontier molecular orbitals and corresponding energy levels, and calculated $\triangle \mathrm{E}$ of B25 in the ground state in methanol at M062X/Def2SVP level.

Figure S28. a) Molecular structure, b) optimized structure, c) frontier molecular orbitals and corresponding energy levels, and calculated $\triangle \mathrm{E}$ of B26 in the ground state in methanol at M062X/Def2SVP level.

Figure S29. a) Molecular structure, b) optimized structure, c) frontier molecular orbitals and corresponding energy levels, and calculated $\triangle \mathrm{E}$ of B27 in the ground state in methanol at M062X/Def2SVP level.

Figure S30. a) Molecular structure, b) optimized structure, c) frontier molecular orbitals and corresponding energy levels, and calculated $\triangle \mathrm{E}$ of B28 in the ground state in methanol at M062X/Def2SVP level.

Figure S31. a) Molecular structure, b) optimized structure, c) frontier molecular orbitals and corresponding energy levels, and calculated $\triangle \mathrm{E}$ of B29 in the ground state in methanol at M062X/Def2SVP level.

Figure S32. a) Molecular structure, b) optimized structure, c) frontier molecular orbitals and corresponding energy levels, and calculated $\triangle \mathrm{E}$ of $\mathrm{B} 30$ in the ground state in methanol at M062X/Def2SVP level.

Figure S33. a) Molecular structure, b) optimized structure, c) frontier molecular orbitals and corresponding energy levels, and calculated $\triangle \mathrm{E}$ of B31 in the ground state in methanol at M062X/Def2SVP level. 
Figure S34. a) Molecular structure, b) optimized structure, c) frontier molecular orbitals and corresponding energy levels, and calculated $\triangle \mathrm{E}$ of B32 in the ground state in methanol at M062X/Def2SVP level.

Figure S35. a) Molecular structure, b) optimized structure, c) frontier molecular orbitals and corresponding energy levels, and calculated $\Delta \mathrm{E}$ of B33 in the ground state in methanol at M062X/Def2SVP level.

Figure S36. a) Molecular structure, b) optimized structure, c) frontier molecular orbitals and corresponding energy levels, and calculated $\Delta \mathrm{E}$ of B34 in the ground state in methanol at M062X/Def2SVP level.

Figure S37. a) Molecular structure, b) optimized structure, c) frontier molecular orbitals and corresponding energy levels, and calculated $\Delta \mathrm{E}$ of B35 in the ground state in methanol at M062X/Def2SVP level.

Figure S38. a) Molecular structure, b) optimized structure, c) frontier molecular orbitals and corresponding energy levels, and calculated $\triangle \mathrm{E}$ of B36 in the ground state in methanol at M062X/Def2SVP level.

Figure S39. a) Molecular structure, b) optimized structure, c) frontier molecular orbitals and corresponding energy levels, and calculated $\triangle \mathrm{E}$ of B37 in the ground state in methanol at M062X/Def2SVP level.....

Figure S40. a) Molecular structure, b) optimized structure, c) frontier molecular orbitals and corresponding energy levels, and calculated $\Delta \mathrm{E}$ of B38 in the ground state in methanol at M062X/Def2SVP level.

Figure S41. a) Molecular structure, b) optimized structure, c) frontier molecular orbitals and corresponding energy levels, and calculated $\Delta \mathrm{E}$ of B39 in the ground state in methanol at M062X/Def2SVP level.

Figure S42. a) Molecular structure, b) optimized structure, c) frontier molecular orbitals and corresponding energy levels, and calculated $\Delta \mathrm{E}$ of B40 in the ground state in methanol at M062X/Def2SVP level.

Figure S43. a) Molecular structure, b) optimized structure, c) frontier molecular orbitals and corresponding energy levels, and calculated $\Delta \mathrm{E}$ of B41 in the ground state in methanol at M062X/Def2SVP level.

Figure S44. a) Molecular structure, b) optimized structure, c) frontier molecular orbitals and corresponding energy levels, and calculated $\Delta E$ of B42 in the ground state in methanol at M062X/Def2SVP level.

Figure S45. a) Molecular structure, b) optimized structure, c) frontier molecular orbitals and corresponding energy levels, and calculated $\Delta \mathrm{E}$ of B43 in the ground state in methanol at M062X/Def2SVP level.

Figure S46. a) Molecular structure, b) optimized structure, c) frontier molecular orbitals and corresponding energy levels, and calculated $\triangle \mathrm{E}$ of $\mathrm{B} 44$ in the ground state in methanol at M062X/Def2SVP level.

Figure S47. a) Molecular structure, b) optimized structure, c) frontier molecular orbitals and corresponding energy levels, and calculated $\Delta \mathrm{E}$ of B45 in the ground state in methanol at M062X/Def2SVP level.

Figure S48. a) Molecular structure, b) optimized structure, c) frontier molecular orbitals and corresponding energy levels, and calculated $\Delta \mathrm{E}$ of B46 in the ground state in methanol at M062X/Def2SVP level.

Figure S49. a) Molecular structure, b) optimized structure, c) frontier molecular orbitals and corresponding energy levels, and calculated $\Delta \mathrm{E}$ of B47 in the ground state in methanol at M062X/Def2SVP level.

Figure S50. a) Molecular structure, b) optimized structure, c) frontier molecular orbitals and corresponding energy levels, and calculated $\Delta \mathrm{E}$ of B48 in the ground state in methanol at M062X/Def2SVP level. 
Figure S51. a) Molecular structure, b) optimized structure, c) frontier molecular orbitals and corresponding energy levels, and calculated $\Delta \mathrm{E}$ of B49 in the ground state in methanol at M062X/Def2SVP level.

Figure S52. a) Molecular structure, b) optimized structure, c) frontier molecular orbitals and corresponding energy levels, and calculated $\triangle \mathrm{E}$ of B50 in the ground state in acetonitrile at M062X/Def2SVP level.

Figure S53. a) Molecular structure, b) optimized structure, c) frontier molecular orbitals and corresponding energy levels, and calculated $\triangle \mathrm{E}$ of B51 in the ground state in acetonitrile at M062X/Def2SVP level.

Figure S54. a) Molecular structure, b) optimized structure, c) frontier molecular orbitals and corresponding energy levels, and calculated $\triangle \mathrm{E}$ of $\mathrm{B52}$ in the ground state in acetonitrile at M062X/Def2SVP level.

Figure S55. a) Molecular structure, b) optimized structure, c) frontier molecular orbitals and corresponding energy levels, and calculated $\triangle \mathrm{E}$ of B53 in the ground state in acetonitrile at M062X/Def2SVP level.

Figure S56. a) Molecular structure, b) optimized structure, c) frontier molecular orbitals and corresponding energy levels, and calculated $\triangle \mathrm{E}$ of B54 in the ground state in acetonitrile at M062X/Def2SVP level.....

Figure S57. a) Molecular structure, b) optimized structure, c) frontier molecular orbitals and corresponding energy levels, and calculated $\triangle \mathrm{E}$ of B55 in the ground state in acetonitrile at M062X/Def2SVP level.

Figure S58. a) Molecular structure, b) optimized structure, c) frontier molecular orbitals and corresponding energy levels, and calculated $\Delta \mathrm{E}$ of B56 in the ground state in acetonitrile at M062X/Def2SVP level.

Figure S59. a) Molecular structure, b) optimized structure, c) frontier molecular orbitals and corresponding energy levels, and calculated $\triangle \mathrm{E}$ of B57 in the ground state in acetonitrile at M062X/Def2SVP level.

Figure S60. a) Molecular structure, b) optimized structure, c) frontier molecular orbitals and corresponding energy levels, and calculated $\triangle \mathrm{E}$ of $\mathrm{B} 58$ in the ground state in acetonitrile at M062X/Def2SVP level.

Figure S61. a) Molecular structure, b) optimized structure, c) frontier molecular orbitals and corresponding energy levels, and calculated $\triangle \mathrm{E}$ of B59 in the ground state in acetonitrile at M062X/Def2SVP level.

Figure S62. a) Molecular structure, b) optimized structure, c) frontier molecular orbitals and corresponding energy levels, and calculated $\triangle \mathrm{E}$ of $\mathbf{B 6 0}$ in the ground state in acetonitrile at M062X/Def2SVP level.

Figure S63. a) Molecular structure, b) optimized structure, c) frontier molecular orbitals and corresponding energy levels, and calculated $\Delta \mathrm{E}$ of $\mathrm{B} 61$ in the ground state in acetonitrile at M062X/Def2SVP level.

Figure S64. a) Molecular structure, b) optimized structure, c) frontier molecular orbitals and corresponding energy levels, and calculated $\triangle \mathrm{E}$ of $\mathbf{B} 62$ in the ground state in acetonitrile at M062X/Def2SVP level.

Figure S65. a) Molecular structure, b) optimized structure, c) frontier molecular orbitals and corresponding energy levels, and calculated $\triangle \mathrm{E}$ of $\mathrm{B} 63$ in the ground state in acetonitrile at M062X/Def2SVP level.

Figure S66. a) Molecular structure, b) optimized structure, c) frontier molecular orbitals and corresponding energy levels, and calculated $\Delta \mathrm{E}$ of B64 in the ground state in methanol at M062X/Def2SVP level.

Figure S67. a) Molecular structure, b) optimized structure, c) frontier molecular orbitals and corresponding energy levels, and calculated $\triangle \mathrm{E}$ of $\mathbf{B 6 5}$ in the ground state in methanol at M062X/Def2SVP level. 
Figure S68. a) Molecular structure, b) optimized structure, c) frontier molecular orbitals and corresponding energy levels, and calculated $\Delta \mathrm{E}$ of $\mathbf{B 6} 6$ in the ground state in methanol at M062X/Def2SVP level.

Figure S69. a) Molecular structure, b) optimized structure, c) frontier molecular orbitals and corresponding energy levels, and calculated $\triangle \mathrm{E}$ of B67 in the ground state in methanol at M062X/Def2SVP level.

Figure S70. a) Molecular structure, b) optimized structure, c) frontier molecular orbitals and corresponding energy levels, and calculated $\Delta \mathrm{E}$ of $\mathbf{B 6 8}$ in the ground state in methanol at M062X/Def2SVP level.

Figure S71. a) Molecular structure, b) optimized structure, c) frontier molecular orbitals and corresponding energy levels, and calculated $\triangle \mathrm{E}$ of $\mathrm{B} 69$ in the ground state in DMSO at M062X/Def2SVP level.

Figure S72. a) Molecular structure, b) optimized structure, c) frontier molecular orbitals and corresponding energy levels, and calculated $\triangle E$ of $B 70$ in the ground state in water at M062X/Def2SVP level.

Figure S73. a) Molecular structure, b) optimized structure, c) frontier molecular orbitals and corresponding energy levels, and calculated $\triangle \mathrm{E}$ of $\mathrm{B} 71$ in the ground state in water at M062X/Def2SVP level.....

Figure S74. a) Molecular structure, b) optimized structure, c) frontier molecular orbitals and corresponding energy levels, and calculated $\triangle \mathrm{E}$ of $\mathrm{B} 72$ in the ground state in water at M062X/Def2SVP level.

Figure S75. a) Molecular structure, b) optimized structure, c) frontier molecular orbitals and corresponding energy levels, and calculated $\Delta \mathrm{E}$ of $\mathrm{B} 73$ in the ground state in acetonitrile at M062X/Def2SVP level.

Figure S76. a) Molecular structure, b) optimized structure, c) frontier molecular orbitals and corresponding energy levels, and calculated $\Delta \mathrm{E}$ of B74 in the ground state in acetonitrile at M062X/Def2SVP level.

Figure S77. a) Molecular structure, b) optimized structure, c) frontier molecular orbitals and corresponding energy levels, and calculated $\triangle \mathrm{E}$ of $\mathrm{B75}$ in the ground state in acetonitrile at M062X/Def2SVP level.

Figure S78. a) Molecular structure, b) optimized structure, c) frontier molecular orbitals and corresponding energy levels, and calculated $\triangle E$ of B76 in the ground state in PBS at M062X/Def2SVP level.

Figure S79. a) Molecular structure, b) optimized structure, c) frontier molecular orbitals and corresponding energy levels, and calculated $\triangle \mathrm{E}$ of $\mathrm{B} 77$ in the ground state in acetonitrile at M062X/Def2SVP level.

Figure S80. a) Molecular structure, b) optimized structure, c) frontier molecular orbitals and corresponding energy levels, and calculated $\Delta \mathrm{E}$ of $\mathrm{B} 78$ in the ground state in acetonitrile at M062X/Def2SVP level.

Figure S81. a) Molecular structure, b) optimized structure, c) frontier molecular orbitals and corresponding energy levels, and calculated $\triangle \mathrm{E}$ of $\mathrm{B} 79$ in the ground state in acetonitrile at M062X/Def2SVP level.

Figure S82. a) Molecular structure, b) optimized structure, c) frontier molecular orbitals and corresponding energy levels, and calculated $\Delta \mathrm{E}$ of $\mathbf{B 8 0}$ in the ground state in acetonitrile at M062X/Def2SVP level.

Figure S83. a) Molecular structure, b) optimized structure, c) frontier molecular orbitals and corresponding energy levels, and calculated $\Delta \mathrm{E}$ of $\mathbf{B 8 1}$ in the ground state in methanol at M062X/Def2SVP level.

Figure S84. a) Molecular structure, b) optimized structure, c) frontier molecular orbitals and corresponding energy levels, and calculated $\triangle \mathrm{E}$ of $\mathbf{B 8 2}$ in the ground state in methanol at M062X/Def2SVP level. 
Figure S85. a) Molecular structure, b) optimized structure, c) frontier molecular orbitals and corresponding energy levels, and calculated $\triangle E$ of B83 in the ground state in PBS at

M062X/Def2SVP level.

Figure S86. The histogram and accumulated probability of quantum yields for BODIPY dyes B1-

B83 in polar solvents with $(\mathrm{a}, \mathrm{b}) \Delta \mathrm{E}<0.6 \mathrm{eV}$ and $(\mathrm{c}, \mathrm{d}) \Delta \mathrm{E}>0.6 \mathrm{eV}$.

Figure S87. Correlation between quantum yields and calculated $\Delta \mathrm{E}$ at $\omega B 97 X D / D e f 2 S V P$ level for

meso-phenyl substituted BODIPY compounds B1-B83 in polar solvents.

S89

Figure S88. The frontier molecular orbitals and corresponding $\triangle \mathrm{E}$ of $\mathbf{B 6 9}, \mathbf{S 1}$, and S2, calculated in the ground state in DMSO at M062X/Def2SVP level. The distances between the fluorophore and

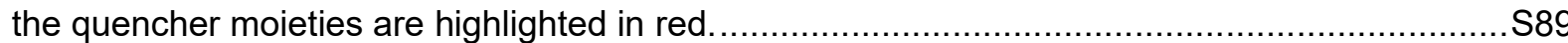

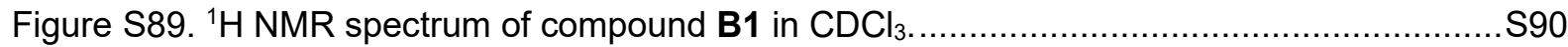

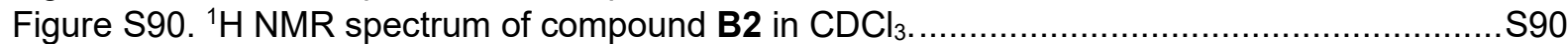

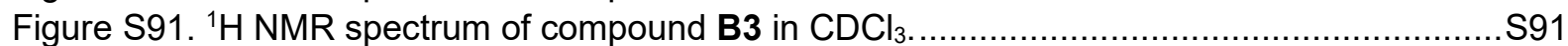

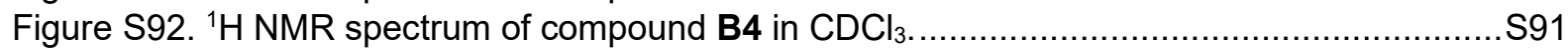

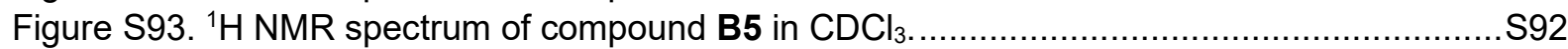

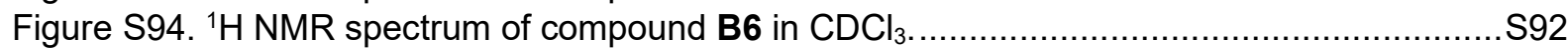

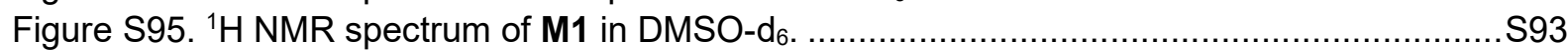

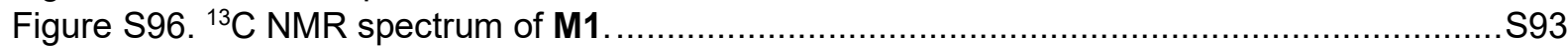

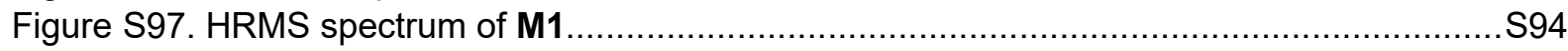

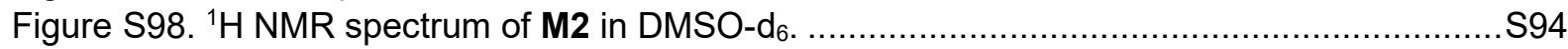

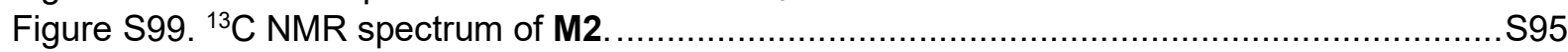

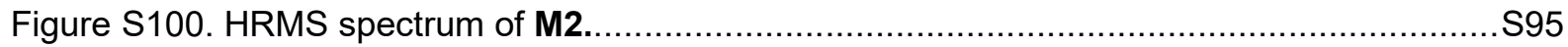

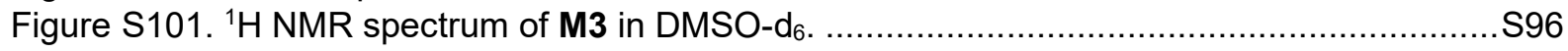

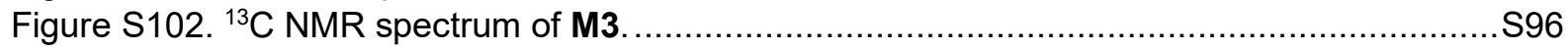

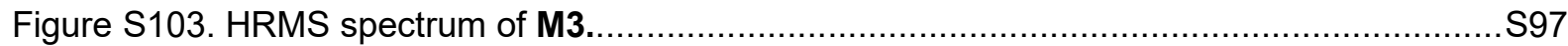

Figure S104. Normalized UV-Vis absorption spectra of B1 in hexane, dichloromethane, ethyl

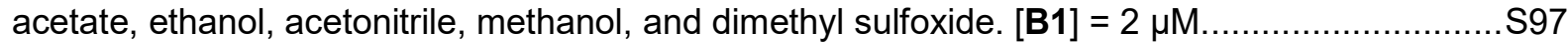

Figure S105. Normalized fluorescence spectra of B1 in hexane, dichloromethane, ethyl acetate, ethanol, acetonitrile, methanol, and dimethyl sulfoxide. $[\mathrm{B} 1]=2 \mu \mathrm{M}$; excitation wavelength $=470$

nm.

Figure S106. Normalized UV-Vis absorption spectra of B2 in hexane, dichloromethane, ethyl acetate, ethanol, acetonitrile, methanol, and dimethyl sulfoxide. [B2] $=2 \mu \mathrm{M}$.

Figure S107. Normalized fluorescence spectra of B2 in hexane, dichloromethane, ethyl acetate, ethanol, acetonitrile, methanol, and dimethyl sulfoxide. [B2] $=2 \mu \mathrm{M}$; excitation wavelength $=470$

$\mathrm{nm}$.

Figure S108. Normalized UV-Vis absorption spectra of B3 in hexane, dichloromethane, ethyl acetate, ethanol, acetonitrile, methanol, and dimethyl sulfoxide. [B3] $=2 \mu \mathrm{M}$......

Figure S109. Normalized fluorescence spectra of B3 in hexane, dichloromethane, ethyl acetate, ethanol, acetonitrile, methanol, and dimethyl sulfoxide. [B3] $=2 \mu \mathrm{M}$; excitation wavelength $=470$

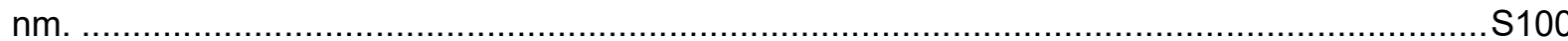

Figure S110. Normalized UV-Vis absorption spectra of B4 in hexane, dichloromethane, ethyl

acetate, ethanol, acetonitrile, methanol, and dimethyl sulfoxide. [B4] $=2 \mu \mathrm{M} \ldots \ldots \ldots \ldots \ldots \ldots \ldots \ldots . . . . . . . . . . . . .100$

Figure S111. Normalized fluorescence spectra of B4 in hexane, dichloromethane, ethyl acetate, ethanol, acetonitrile, methanol, and dimethyl sulfoxide. [B4] $=2 \mu \mathrm{M}$; excitation wavelength $=470$

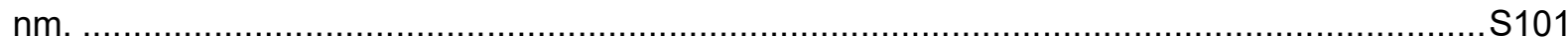

Figure S112. Normalized UV-Vis absorption spectra of B5 in hexane, dichloromethane, ethyl acetate, ethanol, acetonitrile, methanol, and dimethyl sulfoxide. [B5] = $2 \mu \mathrm{M} \ldots \ldots \ldots \ldots \ldots \ldots \ldots \ldots . . . . . . . . . . . . . .101$ Figure S113. Normalized fluorescence spectra of B5 in hexane, dichloromethane, ethyl acetate, ethanol, acetonitrile, methanol, and dimethyl sulfoxide. [B5] = $2 \mu \mathrm{M}$; excitation wavelength $=470$ $\mathrm{nm}$.

Figure S114. Normalized UV-Vis absorption spectra of B6 in hexane, dichloromethane, ethyl acetate, ethanol, acetonitrile, methanol, and dimethyl sulfoxide. [B6] $=2 \mu \mathrm{M} \ldots \ldots \ldots \ldots \ldots \ldots \ldots \ldots . . . . . . . . . . . . .102$ 
Figure S115. Normalized fluorescence spectra of B6 in hexane, dichloromethane, ethyl acetate, ethanol, acetonitrile, methanol, and dimethyl sulfoxide. $[\mathrm{B} 6]=2 \mu \mathrm{M}$; excitation wavelength $=470$ $\mathrm{nm}$.

Figure S116. Histogram of the dihedral angle, $\theta$, in tetramethyl BODIPY dyes, as searched from the Cambridge Crystallographic Data Centre (CCDC); the inset shows the search moiety used in the data-mining, and the probability, $\mathrm{P}$, of $\theta$ being greater than $95^{\circ}$ or smaller than $85^{\circ}$.

Figure S117. Calibration curve of the viscosity $(n)$ of ethanol-glycerol mixture at $21^{\circ} \mathrm{C}$, as a function of (a) the molar fraction of ethanol, $x(\%)$; and (b) the volume fraction of ethanol, $v(\%) .50$ cP corresponds to $\sim 50 \%$ of molar fraction and $\sim 45 \%$ of volume fraction of ethanol. The draw data for constructing this calibration curve were extracted from Reference 36.

Figure S118. a) Co-staining of HeLa cells using B5 $(1 \mu \mathrm{M})$ and LD $540(1 \mu \mathrm{M})$; green channel, B5; red channel, LD 540; yellow channel, the merged image. b) Co-staining of HeLa cells using B6 (1 $\mu \mathrm{M})$ and LD $540(1 \mu \mathrm{M})$; green channel, B6; red channel, LD 540; yellow channel, the merged

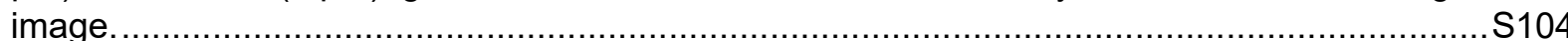
Figure S119. Lipid droplet dynamics of HeLa cells, as revealed by B4...............................S105 Figure S120. UV-Vis absorption spectra (a) and fluorescence intensity changes (b) of B4 as a function of viscosity in the mixture of ethanol and glycerol. [B4] $=2 \mu \mathrm{M}$; excitation wavelength $=$ $470 \mathrm{~nm}$.

Figure S121. UV-Vis absorption spectra (a) and fluorescence intensity changes (b) of B5 as a function of viscosity in the mixture of ethanol and glycerol. [B5] $=2 \mu \mathrm{M}$; excitation wavelength = $470 \mathrm{~nm}$.

Figure S122. UV-Vis absorption spectra (a) and fluorescence intensity changes (b) of B6 as a function of viscosity in the mixture of ethanol and glycerol. [B6] $=2 \mu \mathrm{M}$; excitation wavelength = $470 \mathrm{~nm}$. S106

Figure S123. a) Molecular structure, b) optimized structure, c) frontier molecular orbitals and corresponding energy levels, and calculated $\Delta \mathrm{E}$ of $\mathbf{R} \mathbf{1}$ in the ground state in water at M062X/Def2SVP level.

Figure S124. a) Molecular structure, b) optimized structure, c) frontier molecular orbitals and corresponding energy levels, and calculated $\Delta \mathrm{E}$ of $\mathbf{R} 2$ in the ground state in water at M062X/Def2SVP level.

Figure S125. a) Molecular structure, b) optimized structure, c) frontier molecular orbitals and corresponding energy levels, and calculated $\Delta E$ of $\mathbf{R} 3$ in the ground state in water at M062X/Def2SVP level.

Figure S126. a) Molecular structure, b) optimized structure, c) frontier molecular orbitals and corresponding energy levels, and calculated $\Delta \mathrm{E}$ of $\mathbf{R 4}$ in the ground state in water at M062X/Def2SVP level......

Figure S127. a) Molecular structure, b) optimized structure, c) frontier molecular orbitals and corresponding energy levels, and calculated $\Delta \mathrm{E}$ of $\mathbf{R 5}$ in the ground state in water at M062X/Def2SVP level.

Figure S128. a) Molecular structure, b) optimized structure, c) frontier molecular orbitals and corresponding energy levels, and calculated $\Delta \mathrm{E}$ of $\mathbf{R} \mathbf{6}$ in the ground state in water at M062X/Def2SVP level.

Figure S129. a) Molecular structure, b) optimized structure, c) frontier molecular orbitals and corresponding energy levels, and calculated $\Delta \mathrm{E}$ of $\mathbf{R 7}$ in the ground state in water at M062X/Def2SVP level

Figure S130. a) Molecular structure, b) optimized structure, c) frontier molecular orbitals and corresponding energy levels, and calculated $\triangle \mathrm{E}$ of $\mathbf{R} \mathbf{8}$ in the ground state in PBS at M062X/Def2SVP level.

Figure S131. a) Molecular structure, b) optimized structure, c) frontier molecular orbitals and corresponding energy levels, and calculated $\triangle \mathrm{E}$ of $\mathrm{R} 9$ in the ground state in PBS at M062X/Def2SVP level. 
Figure S132. a) Molecular structure, b) optimized structure, c) frontier molecular orbitals and corresponding energy levels, and calculated $\triangle E$ of R10 in the ground state in PBS at M062X/Def2SVP level.

Figure S133. a) Molecular structure, b) optimized structure, c) frontier molecular orbitals and corresponding energy levels, and calculated $\triangle \mathrm{E}$ of R11 in the ground state in PBS at M062X/Def2SVP level.

Figure S134. a) Molecular structure, b) optimized structure, c) frontier molecular orbitals and corresponding energy levels, and calculated $\triangle E$ of R12 in the ground state in PBS at M062X/Def2SVP level.

Figure S135. a) Molecular structure, b) optimized structure, c) frontier molecular orbitals and corresponding energy levels, and calculated $\triangle E$ of R13 in the ground state in PBS at M062X/Def2SVP level.

Figure S136. a) Molecular structure, b) optimized structure, c) frontier molecular orbitals and corresponding energy levels, and calculated $\triangle E$ of R14 in the ground state in PBS at M062X/Def2SVP level.

Figure S137. a) Molecular structure, b) optimized structure, c) frontier molecular orbitals and corresponding energy levels, and calculated $\triangle \mathrm{E}$ of R15 in the ground state in PBS at M062X/Def2SVP level.

Figure S138. a) Molecular structure, b) optimized structure, c) frontier molecular orbitals and corresponding energy levels, and calculated $\triangle \mathrm{E}$ of R16 in the ground state in PBS at M062X/Def2SVP level.

Figure S139. a) Molecular structure, b) optimized structure, c) frontier molecular orbitals and corresponding energy levels, and calculated $\Delta \mathrm{E}$ of $\mathrm{R} 17$ in the ground state in water at M062X/Def2SVP level.

Figure S140. a) Molecular structure, b) optimized structure, c) frontier molecular orbitals and corresponding energy levels, and calculated $\Delta \mathrm{E}$ of R18 in the ground state in water at M062X/Def2SVP level.

Figure S141. a) Molecular structure, b) optimized structure, c) frontier molecular orbitals and corresponding energy levels, and calculated $\Delta \mathrm{E}$ of R19 in the ground state in water at M062X/Def2SVP level.

Figure S142. a) Molecular structure, b) optimized structure, c) frontier molecular orbitals and corresponding energy levels, and calculated $\Delta \mathrm{E}$ of $\mathbf{R 2 0}$ in the ground state in water at M062X/Def2SVP level.

Figure S143. a) Molecular structure, b) optimized structure, c) frontier molecular orbitals and corresponding energy levels, and calculated $\triangle \mathrm{E}$ of $\mathbf{R} 21$ in the ground state in water at M062X/Def2SVP level.

Figure S144. a) Molecular structure, b) optimized structure, c) frontier molecular orbitals and corresponding energy levels, and calculated $\Delta \mathrm{E}$ of $\mathbf{R 2 2}$ in the ground state in water at M062X/Def2SVP level

Figure S145. a) Molecular structure, b) optimized structure, c) frontier molecular orbitals and corresponding energy levels, and calculated $\triangle \mathrm{E}$ of $\mathbf{R 2 3}$ in the ground state in water at M062X/Def2SVP level.

Figure S146. a) Molecular structure, b) optimized structure, c) frontier molecular orbitals and corresponding energy levels, and calculated $\triangle \mathrm{E}$ of R24 in the ground state in PBS at M062X/Def2SVP level.

Figure S147. a) Molecular structure, b) optimized structure, c) frontier molecular orbitals and corresponding energy levels, and calculated $\triangle \mathrm{E}$ of $\mathbf{R 2 5}$ in the ground state in PBS at M062X/Def2SVP level.

Figure S148. a) Molecular structure, b) optimized structure, c) frontier molecular orbitals and corresponding energy levels, and calculated $\triangle E$ of R26 in the ground state in PBS at M062X/Def2SVP level. 
Figure S149. a) Molecular structure, b) optimized structure, c) frontier molecular orbitals and corresponding energy levels, and calculated $\triangle \mathrm{E}$ of R27 in the ground state in ethanol at M062X/Def2SVP level.

Figure S150. a) Molecular structure, b) optimized structure, c) frontier molecular orbitals and corresponding energy levels, and calculated $\Delta \mathrm{E}$ of $\mathbf{R 2 8}$ in the ground state in ethanol at M062X/Def2SVP level.

Figure S151. a) Molecular structure, b) optimized structure, c) frontier molecular orbitals and corresponding energy levels, and calculated $\triangle \mathrm{E}$ of $\mathrm{F} 1$ in the ground state in PBS at M062X/Def2SVP level.

Figure S152. a) Molecular structure, b) optimized structure, c) frontier molecular orbitals and corresponding energy levels, and calculated $\triangle \mathrm{E}$ of $\mathbf{F 2}$ in the ground state in PBS at M062X/Def2SVP level.

Figure S153. a) Molecular structure, b) optimized structure, c) frontier molecular orbitals and corresponding energy levels, and calculated $\triangle E$ of $F 3$ in the ground state in PBS at M062X/Def2SVP level.

Figure S154. a) Molecular structure, b) optimized structure, c) frontier molecular orbitals and corresponding energy levels, and calculated $\triangle \mathrm{E}$ of F4 in the ground state in PBS at M062X/Def2SVP level.

Figure S155. a) Molecular structure, b) optimized structure, c) frontier molecular orbitals and corresponding energy levels, and calculated $\triangle \mathrm{E}$ of $\mathbf{F 5}$ in the ground state in PBS at M062X/Def2SVP level.

Figure S156. a) Molecular structure, b) optimized structure, c) frontier molecular orbitals and corresponding energy levels, and calculated $\Delta \mathrm{E}$ of $\mathbf{F} \mathbf{6}$ in the ground state in water at M062X/Def2SVP level.

Figure S157. a) Molecular structure, b) optimized structure, c) frontier molecular orbitals and corresponding energy levels, and calculated $\Delta \mathrm{E}$ of $\mathbf{F} 7$ in the ground state in water at M062X/Def2SVP level.

Figure S158. a) Molecular structure, b) optimized structure, c) frontier molecular orbitals and corresponding energy levels, and calculated $\Delta \mathrm{E}$ of $\mathbf{F 8}$ in the ground state in water at M062X/Def2SVP level.

Figure S159. a) Molecular structure, b) optimized structure, c) frontier molecular orbitals and corresponding energy levels, and calculated $\Delta \mathrm{E}$ of $\mathrm{F} 9$ in the ground state in water at M062X/Def2SVP level.

Figure S160. a) Molecular structure, b) optimized structure, c) frontier molecular orbitals and corresponding energy levels, and calculated $\Delta \mathrm{E}$ of F10 in the ground state in water at M062X/Def2SVP level.

Figure S161. a) Molecular structure, b) optimized structure, c) frontier molecular orbitals and corresponding energy levels, and calculated $\Delta E$ of F11 in the ground state in water at M062X/Def2SVP level.

Figure S162. a) Molecular structure, b) optimized structure, c) frontier molecular orbitals and corresponding energy levels, and calculated $\triangle \mathrm{E}$ of F12 in the ground state in water at M062X/Def2SVP level.

Figure S163. a) Molecular structure, b) optimized structure, c) frontier molecular orbitals and corresponding energy levels, and calculated $\triangle \mathrm{E}$ of F13 in the ground state in water at M062X/Def2SVP level.

Figure S164. a) Molecular structure, b) optimized structure, c) frontier molecular orbitals and corresponding energy levels, and calculated $\Delta \mathrm{E}$ of F14 in the ground state in water at M062X/Def2SVP level.

Figure S165. a) Molecular structure, b) optimized structure, c) frontier molecular orbitals and corresponding energy levels, and calculated $\triangle \mathrm{E}$ of F15 in the ground state in water at M062X/Def2SVP level. 
Figure S166. a) Molecular structure, b) optimized structure, c) frontier molecular orbitals and corresponding energy levels, and calculated $\Delta \mathrm{E}$ of F16 in the ground state in water at M062X/Def2SVP level.

Figure S167. a) Molecular structure, b) optimized structure, c) frontier molecular orbitals and corresponding energy levels, and calculated $\Delta \mathrm{E}$ of F17 in the ground state in water at M062X/Def2SVP level.

Figure S168. a) Molecular structure, b) optimized structure, c) frontier molecular orbitals and corresponding energy levels, and calculated $\Delta \mathrm{E}$ of F18 in the ground state in water at M062X/Def2SVP level.

Figure S169. a) Molecular structure, b) optimized structure, c) frontier molecular orbitals and corresponding energy levels, and calculated $\Delta \mathrm{E}$ of F19 in the ground state in water at M062X/Def2SVP level.

Figure S170. a) Molecular structure, b) optimized structure, c) frontier molecular orbitals and corresponding energy levels, and calculated $\triangle \mathrm{E}$ of F20 in the ground state in water at M062X/Def2SVP level.

Figure S171. a) Molecular structure, b) optimized structure, c) frontier molecular orbitals and corresponding energy levels, and calculated $\Delta \mathrm{E}$ of F21 in the ground state in water at M062X/Def2SVP level.....

Figure S172. a) Molecular structure, b) optimized structure, c) frontier molecular orbitals and corresponding energy levels, and calculated $\Delta \mathrm{E}$ of F22 in the ground state in water at M062X/Def2SVP level.

Figure S173. a) Molecular structure, b) optimized structure, c) frontier molecular orbitals and corresponding energy levels, and calculated $\triangle \mathrm{E}$ of F23 in the ground state in PBS at M062X/Def2SVP level.

Figure S174. a) Molecular structure, b) optimized structure, c) frontier molecular orbitals and corresponding energy levels, and calculated $\triangle \mathrm{E}$ of F24 in the ground state in PBS at M062X/Def2SVP level.

Figure S175. a) Molecular structure, b) optimized structure, c) frontier molecular orbitals and corresponding energy levels, and calculated $\triangle \mathrm{E}$ of F25 in the ground state in PBS at M062X/Def2SVP level.

Figure S176. a) Molecular structure, b) optimized structure, c) frontier molecular orbitals and corresponding energy levels, and calculated $\triangle E$ of F26 in the ground state in PBS at M062X/Def2SVP level.

Figure S177. a) Molecular structure, b) optimized structure, c) frontier molecular orbitals and corresponding energy levels, and calculated $\triangle E$ of F27 in the ground state in PBS at M062X/Def2SVP level.

Figure S178. a) Molecular structure, b) optimized structure, c) frontier molecular orbitals and corresponding energy levels, and calculated $\triangle E$ of $\mathbf{F 2 8}$ in the ground state in PBS at M062X/Def2SVP level.

Figure S179. a) Molecular structure, b) optimized structure, c) frontier molecular orbitals and corresponding energy levels, and calculated $\triangle E$ of F29 in the ground state in PBS at M062X/Def2SVP level.

Figure S180. The histogram and accumulated probability of quantum yields for fluorescein, rhodamine and Si-rhodamine derivatives in polar solvents with (a, b) $\Delta \mathrm{E}<0.6 \mathrm{eV}$ and (c, d) $\Delta \mathrm{E}\rangle$

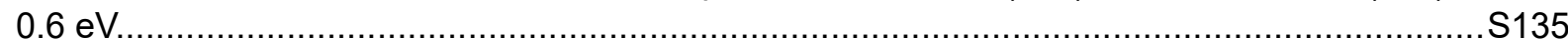

Figure S181. a) Molecular structure, b) optimized structure, c) frontier molecular orbitals and corresponding energy levels, and calculated $\Delta \mathrm{E}$ of $\mathbf{M} 1$ in the ground state in methanol at M062X/Def2SVP level.

Figure S182. a) Molecular structure, b) optimized structure, c) frontier molecular orbitals and corresponding energy levels, and calculated $\triangle \mathrm{E}$ of $\mathbf{M 2}$ in the ground state in methanol at M062X/Def2SVP level. 
Figure S183. a) Molecular structure, b) optimized structure, c) frontier molecular orbitals and corresponding energy levels, and calculated $\triangle \mathrm{E}$ of $\mathbf{M} 3$ in the ground state in methanol at M062X/Def2SVP level.

Figure S184. Normalized UV-Vis absorption spectra of M1 in dichloromethane, ethyl acetate, ethanol, acetonitrile, methanol, and dimethyl sulfoxide solutions. [M1] $=2 \mu \mathrm{M}$.

Figure S185. Normalized UV-Vis absorption spectra of M2 in dichloromethane, ethyl acetate,

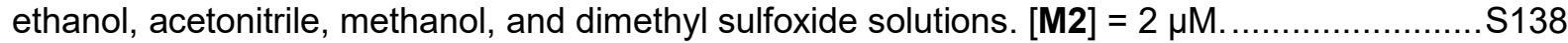
Figure S186. Normalized UV-Vis absorption spectra of M3 in dichloromethane, ethyl acetate, ethanol, acetonitrile, methanol, and dimethyl sulfoxide solutions. [M3] $=2 \mu \mathrm{M}$. S139 Figure S187. Normalized fluorescence spectra of $\mathbf{M 1}$ in dichloromethane, ethyl acetate, ethanol, acetonitrile, methanol, and dimethyl sulfoxide. [M1] $=2 \mu \mathrm{M}$; excitation wavelength $=520 \mathrm{~nm}$...S139 Figure S188. Normalized fluorescence spectra of $\mathbf{M} 2$ in dichloromethane, ethyl acetate, ethanol, acetonitrile, methanol, and dimethyl sulfoxide. [M2] $=2 \mu \mathrm{M}$; excitation wavelength $=520 \mathrm{~nm}$...S140 Figure S189. Normalized fluorescence spectra of $\mathbf{M} 3$ in dichloromethane, ethyl acetate, ethanol, acetonitrile, methanol, and dimethyl sulfoxide. [M3] = $2 \mu \mathrm{M}$; excitation wavelength $=520 \mathrm{~nm}$...S140 Figure S190. Co-staining of HeLa cells using $\mathbf{M} 3(3 \mu \mathrm{M})$ and Cy5(2-((1E,3E-5E)-5-1,3,3trimethylindolin-2-ylidene)penta-1,3-dien-1-ylidene)- 1,3,3-trimethyl -3H-indol-1-ium iodide; 500 nM). Green channel, M3; red channel, Cy5; yellow channel, the merged image. Figure S191. Stained live HeLa cell using M1 $(3 \mu \mathrm{M})$, with significant noises from cytoplasm in addition to emissions from mitochondria.

\section{LIST OF TABLES}

Table S1. Quantum yields of meso-phenyl substituted BODIPY dyes in polar solvents.. S21 Table S2. Calculated $\triangle E$ values of BODIPY dyes at $\omega B$ 97XD/Def2SVP level.

Table S3. Reference codes and dihedral angles returned from the structural search of tetramethyl BODIPY dyes in CCDC.

Table S4. Molecular structures, calcuated $\Delta \mathrm{E}(\mathrm{eV})$, UV-Vis absorption peaks $\left(\lambda_{\mathrm{abs}} ; \mathrm{nm}\right)$, emission peaks ( $\lambda_{\text {emi; }}, \mathrm{nm}$ ), and quantum yields (QY) of meso-phenyl substituted BODIPY dyes in various solvents. S38

Table S5. Quantum yields of meso-phenyl substituted O/Si-rhodamine dyes in polar solvents

Table S6. Quantum yields of meso-phenyl substituted fluorescein dyes in polar solvents.

Table S7. Molecular structures, calculated $\Delta \mathrm{E}(\mathrm{eV})$, UV-Vis absorption peaks $\left(\lambda_{\mathrm{abs}} ; \mathrm{nm}\right)$, emission peaks ( $\lambda_{\text {emi; }} \mathrm{nm}$ ), and quantum yields (QY) of $\mathbf{M 1}, \mathbf{M} 2$, and $\mathbf{M} 3$ in various solvents. 


\section{Computational methods}

The density functional theory (DFT) and time-dependent (TD-DFT) were employed in this work. All structure optimizations were performed without constraints using DFT-M062X,${ }^{1}$ and $\omega B$ 97X $D^{2}$ functionals and Def2SVP 3 basis set for the ground states. Solvation effects were taken into account using the SMD model. ${ }^{4}$ Solvents in use are in accordance with corresponding experimental conditions. Frequency calculations were performed to confirm that we obtained stable structures without imaginary vibrational frequencies. The electronic excitation of all molecules were calculated using state-specific equilibrium solvation (CorrectedLR) ${ }^{5}$ at TDDFTM062X/Def2SVP. We chose the M062X functional, as this functional has been recommended for applications involving the structural optimizations of organic molecules as well as the calculations of electronic excitation energies. ${ }^{1}$ All DFT and TD-DFT calculations were carried out using Gaussian $16 \mathrm{~A} .{ }^{6}$

\section{Solvation model}

SMD and PCM are two representative solvation models. Compared with PCM, SMD not only include the bulk electrostatic contribution arising from a self-consistent reaction field treatment, but also consider the cavitydispersion solvent-structure term, which arises from short-range interactions between the solute and solvent molecules in the first solvation shell. ${ }^{4}$ In this work, we calculated the $\triangle \mathrm{E}$ of B1 - B6 using SMD and PCM model at M062X/Def2SVP level. Our results showed that these two solvation models yield similar results. This indicated that the effect of solvation models is negligible in calculating $\Delta \mathrm{E}$.

a

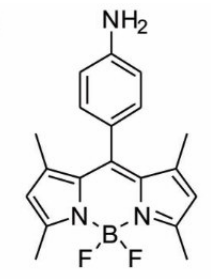

B4 $\varphi=0.001(\mathrm{EtOH})$

b

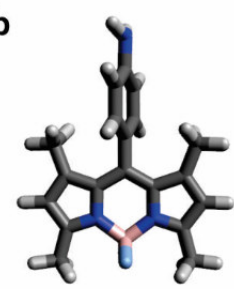

c

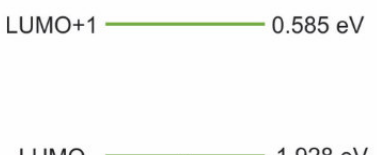

LUMO

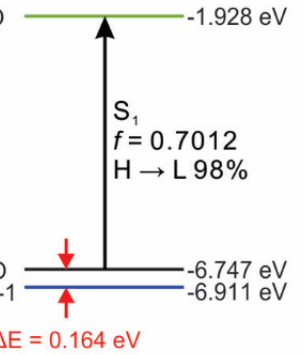

M062X/Def2SVP
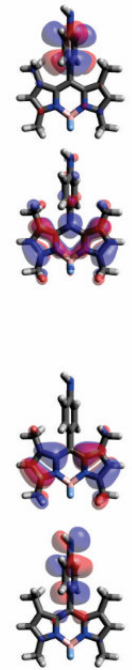

d

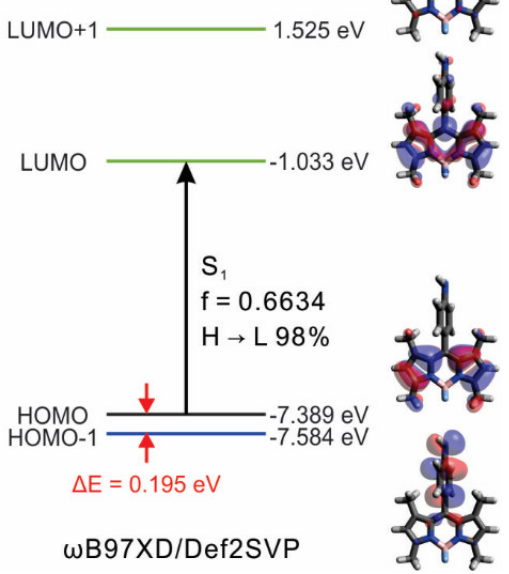

Figure S1. (a) Molecular structure of $p-\mathrm{NH} 2-\mathrm{Ph}-\mathrm{BDP}$ (B4) and its quantum yield in ethanol; (b) optimized geometry of B4 in the ground state, frontier molecular orbitals and corresponding energy levels in the ground state in ethanol at M062X/Def2SVP (c) and wB97XD/Def2SVP (d) levels; $f$ refers to the oscillator strength in the first excited state; $98 \%$ of contribution is from HOMO to LUMO transition. Importantly, the energy level of quenching orbital (represented by a blue line) is lower than that of HOMO. 


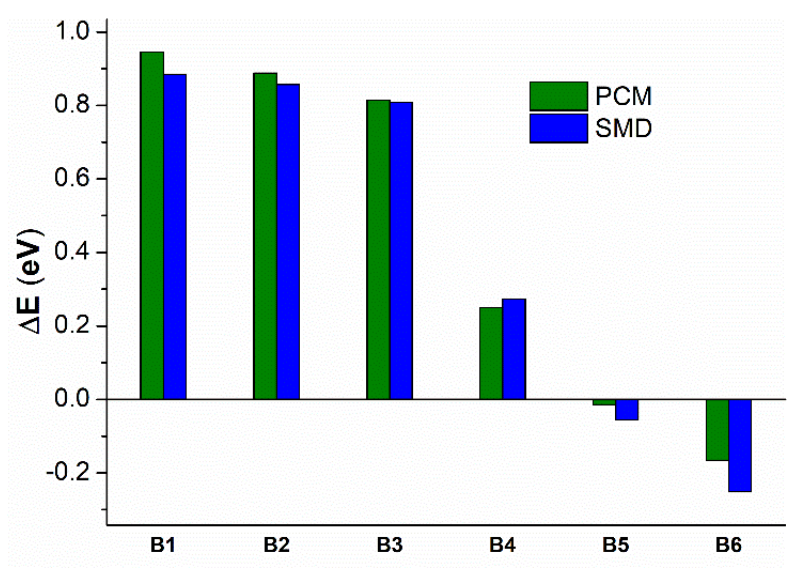

Figure S2. Calculated $\triangle \mathrm{E}$ using PCM and SMD solvation models at M062X/Def2SVP level.

\section{Chemical synthesis and characterizations}

\section{Materials and instruments}

Unless otherwise stated, all reagents were purchased from commercial suppliers (Sigma-Aldrich, J\&K and Aladdin) and used without further purification. Solvents (DMSO, DMF, tetrahydrofuran, dichloromethane, hexane, ethyl acetate, ethyl alcohol, acetone and methanol) were from J\&K and used without further treatment or distillation.

${ }^{1} \mathrm{H}-\mathrm{NMR}$ spectra were recorded on a Bruker 400 spectrometer, using TMS as an internal standard. Chemical shifts were given in ppm and coupling constants $(J)$ in $\mathrm{Hz}$. UV-Vis absorption spectra were collected on an Agilent Cary 60 UV-Vis Spectrophotometer. The blank correction was performed using the respective pure solvent as a reference. Fluorescence measurements were performed on an Agilent CARY Eclipse fluorescence spectrophotometer. Solid-state fluorescence spectra and solid-state PL efficiencies were measured by Steady State and Transient State Fluorescence Spectrometer (HORIBA Scientific/JY Quantamaster 400).

The quantum yields of compounds was determined via the relative determination method, with Rhodamine 6G as a reference compound.

The femtosecond pump-probe transient absorption measurements were performed using a regenerative amplified Ti:Sapphire laser system (Coherent; $800 \mathrm{~nm}, 70 \mathrm{fs}, 6 \mathrm{~mJ} /$ pulse, and $1 \mathrm{kHz}$ repetition rate) as the laser source and a Helios spectrometer (Ultrafast Systems LLC) as the spectrometer. Briefly, the $800 \mathrm{~nm}$ output pulse from the regenerative amplifier was split in two paths with a $50 \%$ beam splitter. The transmitted part was used to pump a TOPAS Optical Parametric Amplifier (OPA) which generated a wavelength-tunable laser pulse from $250 \mathrm{~nm}$ to $2.5 \mu \mathrm{m}$ as pump beam. The reflected $800 \mathrm{~nm}$ beam was split again into two parts. One part with less than $10 \%$ was attenuated with a neutral density filter and focused into a $2 \mathrm{~mm}$ thick sapphire window to generate a white light continuum (WLC) used as probe beam. The probe beam was focused with an $\mathrm{Al}$ parabolic reflector onto the sample. After going through the sample, the probe beam was collimated and then focused into a fibre-coupled spectrometer with CMOS sensors and detected at a frequency of $1 \mathrm{KHz}$. The intensity of the pump pulse used in the experiment was controlled by a variable neutral-density filter wheel. The delay between the pump and probe pulses was controlled by a motorized delay stage. The pump pulses 
were chopped by a synchronized chopper at $500 \mathrm{~Hz}$ and the absorbance change was calculated with two adjacent probe pulses (pump-blocked and pump-unblocked). The samples were placed in $2 \mathrm{~mm}$ cuvettes and measured under ambient conditions. A $490 \mathrm{~nm}$ excitation wavelength of $20 \mu \mathrm{W}$ was used.

Cell culture and image

HeLa cells were grown in DMEM medium supplemented with 10\% FBS (Fetal Bovine Serum) and 1\% antibiotics at $37{ }^{\circ} \mathrm{C}$ in humidified environment of $5 \% \mathrm{CO}_{2}$. HeLa cells were plated on cell culture dish with 0.17 $\mathrm{mm}$ glass and allowed to adhere for 48 hours.

For imaging lipid droplets in HeLa cells, the experimental group was incubated with $1 \mu \mathrm{M}$ probe in DMEM for $15 \mathrm{~min}$ at $37^{\circ} \mathrm{C}$, then directly used for imaging.

For imaging mitochondria in HeLa cells, the experimental group was incubated with $3 \mu \mathrm{M}$ probe in DMEM for $15 \mathrm{~min}$ at $37^{\circ} \mathrm{C}$, then directly used for imaging.

Fluorescence imaging was performed with Laser Scanning Confocal Microscope (Andor iQ 3.2) with 100×oilimmersion objective lens.

Chemical synthesis of BODIPY derivatives

Synthesis of B1

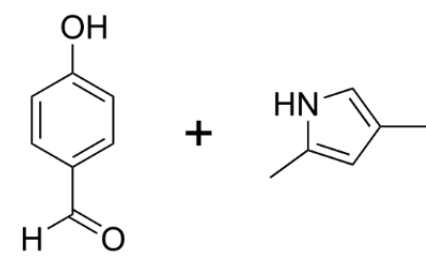

(1) DCM, TFA, R.T., overnight (2) DDQ, R.T., $3 \mathrm{~h}$

(3) $\mathrm{BF}_{3} \cdot \mathrm{Et}_{2} \mathrm{O}, \mathrm{TEA}, \mathrm{R} . \mathrm{T}$., overnight

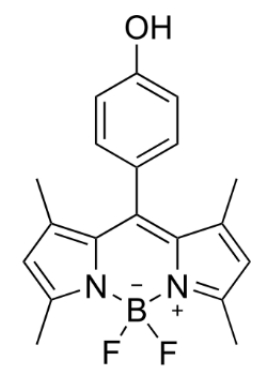

B1

To the solution of 4-hydroxybenzaldehyde (105 mg, $0.86 \mathrm{mmol})$ and 2,4-dimethyl-1H-pyrrole $(200 \mu \mathrm{L}, 1.9 \mathrm{mmol})$ in $5 \mathrm{~mL}$ dry dichloromethane, few drops of trifluoroacetic acid were added. The mixture was stirred overnight at room temperature. 2,3-dichloro-5,6-dicyano-1,4-benzoquinone (195 mg, $0.86 \mathrm{mmol}$ ) was added in $10 \mathrm{~min}$ under ice bath. The solution was stirred for an additional $3 \mathrm{~h}$ at room temperature. Triethylamine $(5 \mathrm{~mL}, 36$ $\mathrm{mmol}$ ) was added, followed by the slow addition of $\mathrm{BF}_{3} \cdot \mathrm{Et}_{2} \mathrm{O}(5 \mathrm{~mL}, 40.5 \mathrm{mmol})$. After stirring overnight at room temperature, the reaction mixture was washed with saturated aqueous $\mathrm{Na}_{2} \mathrm{CO}_{3}$ solution $(3 \times 25 \mathrm{~mL})$, dried over $\mathrm{Na}_{2} \mathrm{SO}_{4}$, and concentrated on a rotary evaporator. The brown, oily residue was purified by column chromatography (PE:DCM $=5: 1-1: 1)$ to give orange solid of $99 \mathrm{mg}$, yield $34 \%$. ${ }^{1} \mathrm{H} \mathrm{NMR}\left(400 \mathrm{MHz}, \mathrm{CDCl}_{3}\right) \delta$ $7.13(\mathrm{~d}, J=7.9 \mathrm{~Hz}, 1 \mathrm{H}), 6.95(\mathrm{~d}, J=8.0 \mathrm{~Hz}, 1 \mathrm{H}), 5.98(\mathrm{~s}, 1 \mathrm{H}), 5.07(\mathrm{~s}, 1 \mathrm{H}), 2.55(\mathrm{~s}, 1 \mathrm{H}), 1.44(\mathrm{~s}, 1 \mathrm{H})$. 
Synthesis of B2

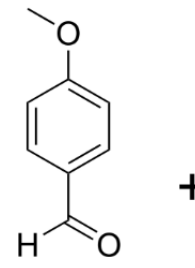

(1) DCM, TFA, R.T., overnight

(2) DDQ, R.T., $3 \mathrm{~h}$

(3) $\mathrm{BF}_{3} \cdot \mathrm{Et}_{2} \mathrm{O}, \mathrm{TEA}, \mathrm{R} . \mathrm{T}$. , overnight

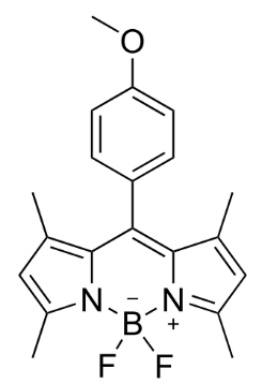

B2

To a solution of 4-methoxybenzaldehyde (105 $\mu \mathrm{L}, 0.86 \mathrm{mmol})$ and 2,4-dimethyl-1H-pyrrole (200 $\mu \mathrm{L}, 1.9 \mathrm{mmol})$ in $5 \mathrm{~mL}$ dry dichloromethane, a few drops of trifluoroacetic acid was added. The mixture was stirred overnight at room temperature. 2,3-dichloro-5,6-dicyano-1,4-benzoquinone (195 mg, $0.86 \mathrm{mmol}$ ) was added in $10 \mathrm{~min}$ under ice bath. The solution was stirred for an additional $3 \mathrm{~h}$ at room temperature. Triethylamine $(5 \mathrm{~mL}, 36$ $\mathrm{mmol}$ ) was added, followed by slow addition of $\mathrm{BF}_{3} \cdot \mathrm{Et}_{2} \mathrm{O}(5 \mathrm{~mL}, 40.5 \mathrm{mmol})$. After stirring overnight at room temperature, the reaction mixture was washed with saturated aqueous $\mathrm{Na}_{2} \mathrm{CO}_{3}$ solution $(3 \times 25 \mathrm{~mL})$, dried over $\mathrm{Na}_{2} \mathrm{SO}_{4}$, and concentrated on a rotary evaporator. The brown, oily residue was purified by column chromatography (PE:DCM = 10:1-3:1) to give an orange solid of $134 \mathrm{mg}$, yield $44 \%$. ${ }^{1} \mathrm{H} \mathrm{NMR}\left(400 \mathrm{MHz}, \mathrm{CDCl}_{3}\right)$ $\delta 7.17(\mathrm{~d}, J=8.6 \mathrm{~Hz}, 2 \mathrm{H}), 7.01$ (d, $J=8.6 \mathrm{~Hz}, 2 \mathrm{H}), 5.97(\mathrm{~s}, 2 \mathrm{H}), 3.87(\mathrm{~s}, 3 \mathrm{H}), 2.55(\mathrm{~s}, 6 \mathrm{H}), 1.43(\mathrm{~s}, 6 \mathrm{H})$.

Synthesis of B3

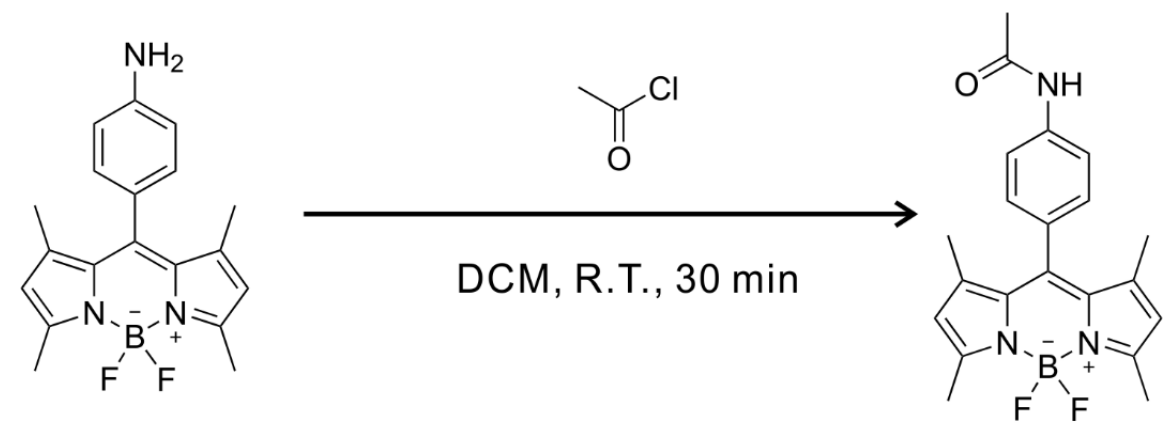

B4

B3

Compound B3 (20 mg, $0.052 \mathrm{mmol})$ was dissolved in $5 \mathrm{~mL}$ dichloromethane, then acetyl chloride $(4 \mu \mathrm{L}, 0.058$ $\mathrm{mmol}$ ) was added to the reaction mixture. The mixture was stirred at room temperature for $30 \mathrm{~min}$, then the solvent was removed under reduced pressure. The residue was further purified by flash column chromatography (PE:EA $=5: 1-1: 2)$ to give an orange solid of $16 \mathrm{mg}$, yield $84 \% .{ }^{1} \mathrm{H} \mathrm{NMR}\left(400 \mathrm{MHz}, \mathrm{CDCl}_{3}\right) \delta$ $7.67(\mathrm{~d}, J=8.4 \mathrm{~Hz}, 2 \mathrm{H}), 7.36(\mathrm{~s}, 1 \mathrm{H}), 7.23(\mathrm{~d}, J=8.4 \mathrm{~Hz}, 2 \mathrm{H}), 5.98(\mathrm{~s}, 2 \mathrm{H}), 2.55(\mathrm{~s}, 6 \mathrm{H}), 2.22(\mathrm{~s}, 3 \mathrm{H}), 1.60(\mathrm{~s}$, $6 \mathrm{H})$.

Synthesis of B4 
<smiles></smiles>

B4

The synthesis of $\mathbf{B} 4$ was according to previous literature reports. ${ }^{7}{ }^{1} \mathrm{H} \mathrm{NMR}\left(400 \mathrm{MHz}, \mathrm{CDCl}_{3}\right) \delta 7.01(\mathrm{~d}, \mathrm{~J}=$ $6.3 \mathrm{~Hz}, 2 \mathrm{H}), 6.79$ (d, J = 6.4 Hz, 2H), 5.97 (s, 2H), 2.71 (s, 2H), 2.54 (s, 6H), 1.49 (s, 6H).

Synthesis of B5
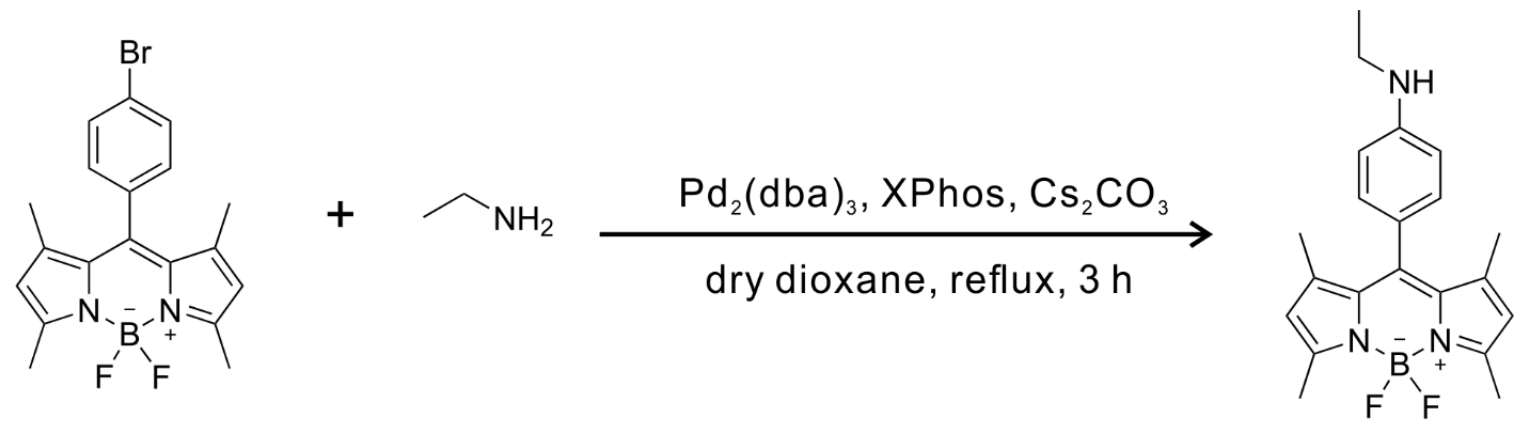

pre-Br

B5

Under an argon atmosphere pre- $\mathrm{Br}(30 \mathrm{mg}, 0.075 \mathrm{mmol}), \mathrm{Pd}_{2}(\mathrm{dba})_{3}(6.8 \mathrm{mg}, 0.0075 \mathrm{mmol})$, XPhos (10.7 mg, $0.023 \mathrm{mmol})$ and $\mathrm{Cs}_{2} \mathrm{CO}_{3}(73 \mathrm{mg}, 0.225 \mathrm{mmol})$ were dissolved in $5 \mathrm{~mL}$ dry dioxane. Ethylamine $(47 \mu \mathrm{L}, 0.75$ $\mathrm{mmol}$ ) was dissolved in $1 \mathrm{~mL}$ dry dioxane and added to the mixture with a syringe. The mixture was stirred at $100{ }^{\circ} \mathrm{C}$ for $3 \mathrm{~h}$. The solvent was then removed under reduced pressure. The residue was further purified by flash column chromatography (PE:DCM $=10: 1-3: 1)$ to give an orange solid of $19 \mathrm{mg}$, yield $70 \% .{ }^{1} \mathrm{H}$ NMR (400 $\left.\mathrm{MHz}, \mathrm{CDCl}_{3}\right) \delta 7.01(\mathrm{~d}, J=8.5 \mathrm{~Hz}, 2 \mathrm{H}), 6.68(\mathrm{~d}, J=8.4 \mathrm{~Hz}, 2 \mathrm{H}), 5.96(\mathrm{~s}, 2 \mathrm{H}), 3.73(\mathrm{~s}, 1 \mathrm{H}), 3.20(\mathrm{q}, J=7.1 \mathrm{~Hz}$, $2 \mathrm{H}), 2.54(\mathrm{~s}, 6 \mathrm{H}), 1.50(\mathrm{~s}, 6 \mathrm{H}), 1.30(\mathrm{t}, J=7.1 \mathrm{~Hz}, 3 \mathrm{H})$.

Synthesis of B6<smiles></smiles>

pre-Br

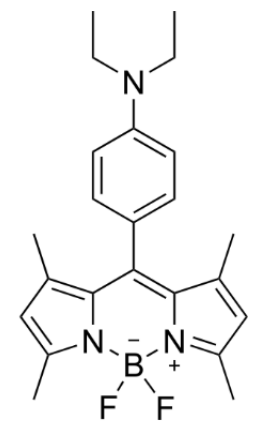

B6 
The synthesis of pre- $\mathrm{Br}$ was according to previous literature reports. Under an argon atmosphere pre- $\mathrm{Br}$ (30 $\mathrm{mg}, 0.075 \mathrm{mmol}), \mathrm{Pd}_{2}(\mathrm{dba})_{3}(6.8 \mathrm{mg}, 0.0075 \mathrm{mmol}), \mathrm{XPhos}(10.7 \mathrm{mg}, 0.023 \mathrm{mmol})$ and $\mathrm{Cs}_{2} \mathrm{CO}_{3}(73 \mathrm{mg}, 0.225$ $\mathrm{mmol}$ ) were dissolved in $5 \mathrm{~mL}$ dry dioxane, diethylamine $(77 \mu \mathrm{L}, 0.75 \mathrm{mmol})$ was dissolved in $1 \mathrm{~mL}$ dry dioxane and added to the mixture by syringe. The mixture was stirred at $100^{\circ} \mathrm{C}$ for $3 \mathrm{~h}$, then the solvent was removed under reduced pressure. The residue was further purified by flash column chromatography (PE:DCM = 10:13:1) to give an orange solid of $21 \mathrm{mg}$, yield $73 \% .{ }^{1} \mathrm{H}$ NMR $\left(400 \mathrm{MHz}, \mathrm{CDCl}_{3}\right) \delta 7.01(\mathrm{~d}, J=8.7 \mathrm{~Hz}, 2 \mathrm{H}), 6.74$ (d, $J=8.7 \mathrm{~Hz}, 2 \mathrm{H}), 5.97(\mathrm{~s}, 2 \mathrm{H}), 3.40(\mathrm{q}, J=7.0 \mathrm{~Hz}, 4 \mathrm{H}), 2.55(\mathrm{~s}, 6 \mathrm{H}), 1.52(\mathrm{~s}, 6 \mathrm{H}), 1.20(\mathrm{t}, J=7.1 \mathrm{~Hz}, 6 \mathrm{H})$.

\section{Chemical synthesis of rhodamine derivatives}

Synthesis of M1

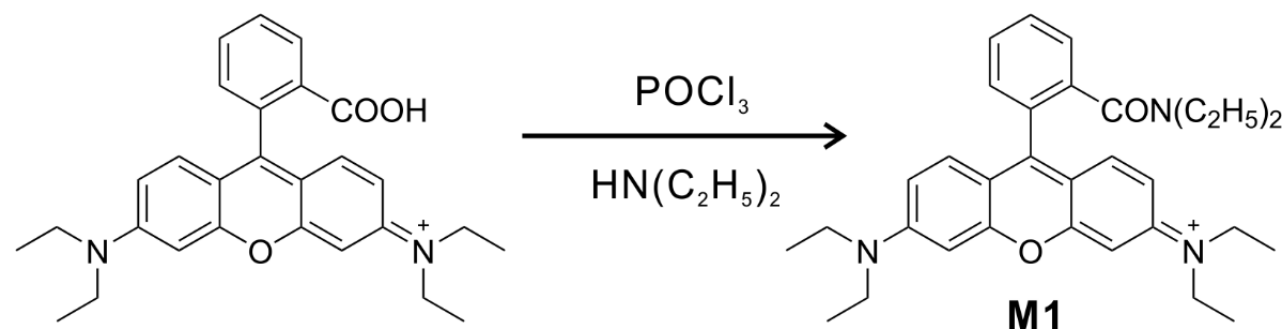

M1 was synthesized according to previous literature reports. The mixture of $\mathrm{RhB}(0.48 \mathrm{~g}, 1 \mathrm{mmol})$ and $\mathrm{POCl}_{3}$ $(280 \mu \mathrm{L}, 3 \mathrm{mmol}$ ) was refluxed in 1,2-dichloroethane $(5 \mathrm{~mL})$ for $2 \mathrm{~h}$, after which the solvent was removed to give dark violet-red oil. The crude acid chloride was dissolved in $\mathrm{CH}_{2} \mathrm{Cl}_{2}(15 \mathrm{~mL})$, then trimethylamine $(0.5 \mathrm{~mL})$ and diethylamine $(103 \mu \mathrm{L}, 1 \mathrm{mmol})$ were dropwise added into the mixture solution. The reaction mixture was stirred at room temperature for $24 \mathrm{~h}$. Then, the solvent was removed, and the crude residue was purified with column chromatography (silica gel, $\mathrm{CH}_{2} \mathrm{Cl}_{2} / \mathrm{CH}_{3} \mathrm{OH}, 10: 1 \mathrm{v} / \mathrm{v}$ ) to give the target compound $\mathbf{M} 1$ as a dark-red semi-solid (0.48 g, 90\%). M1: ${ }^{1} \mathrm{H}$ NMR (400 MHz, DMSO- $\left.d_{6}\right) \delta 7.73$ (dt, $\left.J=7.7,3.9 \mathrm{~Hz}, 2 \mathrm{H}\right), 7.67-7.60(\mathrm{~m}$, $1 \mathrm{H}), 7.56(\mathrm{dd}, J=5.9,3.0 \mathrm{~Hz}, 1 \mathrm{H}), 7.18-7.09(\mathrm{~m}, 4 \mathrm{H}), 6.96(\mathrm{~s}, 2 \mathrm{H}), 3.66(\mathrm{q}, J=6.9 \mathrm{~Hz}, 8 \mathrm{H}), 3.16-3.04(\mathrm{~m}$, $4 \mathrm{H}), 1.21$ (t, $J=7.0 \mathrm{~Hz}, 12 \mathrm{H}), 1.08$ (t, $J=6.9 \mathrm{~Hz}, 3 \mathrm{H}), 0.41$ (t, $J=6.9 \mathrm{~Hz}, 3 \mathrm{H}) .{ }^{13} \mathrm{C}$ NMR (101 MHz, DMSO) $\delta$ 167.52, 157.49, 156.01, 155.60, 137.09, 132.33, 130.70, 130.57, 130.35, 129.74, 126.66, 114.58, 113.54, 96.28, 49.05, 45.84, 43.08, 37.72, 14.11, 12.92, 11.33. LC-MS (ESI): m/z: calcd: 498.3115; found: 498.3078 $[\mathrm{M}]^{+}$.

Synthesis of M3

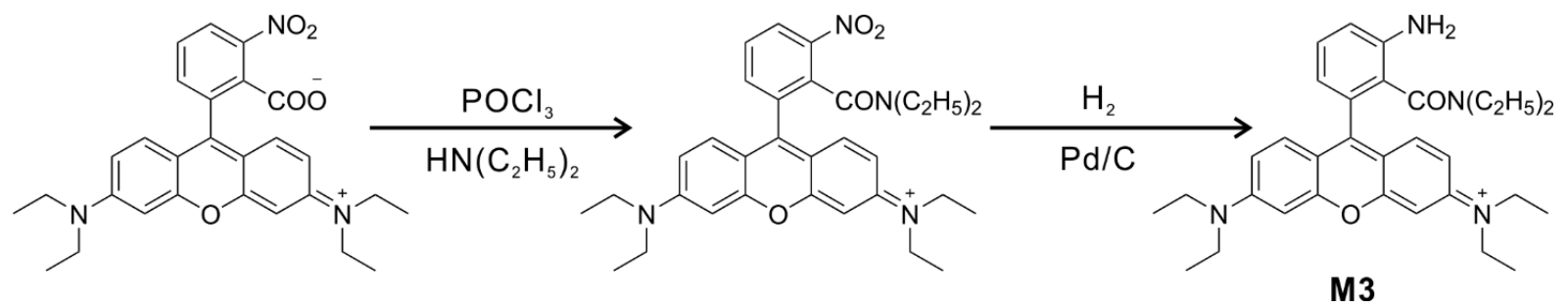

The mixture of 3-nitro- $\mathrm{RhB}(0.57 \mathrm{~g}, 1.2 \mathrm{mmol})$ and $\mathrm{POCl}_{3}(336 \mu \mathrm{L}, 3.6 \mathrm{mmol})$ was refluxed in 1,2-dichloroethane $(15 \mathrm{~mL})$ for $2 \mathrm{~h}$, after which the solvent was removed to give dark violet-red oil. The crude acid chloride was dissolved in $\mathrm{CH}_{2} \mathrm{Cl}_{2}(15 \mathrm{~mL})$, then trimethylamine $(0.9 \mathrm{~mL})$ and diethylamine $(124 \mu \mathrm{L}, 1.2 \mathrm{mmol})$ were dropwise added into the mixture solution. The reaction mixture was stirred at room temperature for $24 \mathrm{~h}$. Then, the 
solvent was removed, and the crude residue was purified with column chromatography (silica gel, $\left.\mathrm{CH}_{2} \mathrm{Cl}_{2} / \mathrm{CH}_{3} \mathrm{OH}, 10: 1 \mathrm{v} / \mathrm{v}\right)$ to give intermediate as a dark-red semi-solid $(0.52 \mathrm{~g}, 75 \%)$. Then, the obtained intermediate $(0.34 \mathrm{~g}, 0.6 \mathrm{mmol})$ was dissolved in $5 \mathrm{~mL}$ methanol and reduced with $34 \mathrm{mg} 10 \% \mathrm{Pd} / \mathrm{C}$ under hydrogen atmosphere. The reaction mixture was filtered, and then the filtrate was evaporated to give M3 as a dark-red semi-solid (0.32 g, 99\%). M3: ${ }^{1} \mathrm{H}$ NMR (400 MHz, DMSO-d6) $\delta 7.38-7.05$ (m, 5H), $7.06-6.82$ (m, $3 \mathrm{H}), 6.59(\mathrm{~d}, \mathrm{~J}=6.5 \mathrm{~Hz}, 1 \mathrm{H}), 5.31(\mathrm{~s}, 2 \mathrm{H}), 3.65(\mathrm{~s}, 8 \mathrm{H}), 3.22(\mathrm{~s}, 2 \mathrm{H}), 2.66(\mathrm{~s}, 2 \mathrm{H}), 1.20$ (s, $12 \mathrm{H}), 0.95(\mathrm{~s}, 3 \mathrm{H})$, 0.30 (s, 3H). LC-MS (ESI): m/z: calcd: 513.3224; found: 513.3252 [M-Cl] $]^{+}$

Synthesis of $\mathbf{M} 2$

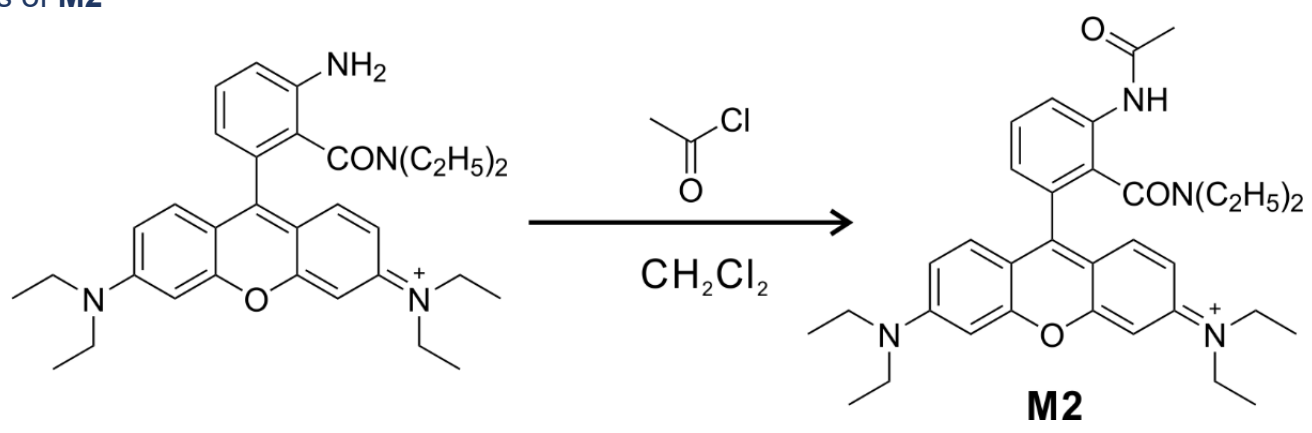

M3 $(0.11 \mathrm{~g}, 0.2 \mathrm{mmol})$ and acetylchloride $(17 \mu \mathrm{L}, 0.24 \mathrm{mmol})$ were mixed in $\mathrm{CH}_{2} \mathrm{Cl}_{2}(2 \mathrm{~mL})$ and stirred for $2 \mathrm{~h}$. Then, the solvent was removed by rotary evaporation. The crude product was purified with column chromatography (silica gel, $\mathrm{CH}_{2} \mathrm{Cl}_{2} / \mathrm{CH}_{3} \mathrm{OH}, 10: 1 \mathrm{v} / \mathrm{v}$ ) to give $\mathbf{M} 2$ as a dark-red semi-solid (0.11 g, $98 \%$ ). $\mathbf{M} 2$ : 1H NMR (400 MHz, DMSO-d $\left.d_{6}\right) 9.66(\mathrm{~s}, 1 \mathrm{H}), 7.84(\mathrm{~s}, 1 \mathrm{H}), 7.65(\mathrm{~s}, 1 \mathrm{H}), 7.44-6.57(\mathrm{~m}, 7 \mathrm{H}), 3.65(\mathrm{~s}, 8 \mathrm{H}), 3.05$ (s, 2H), 2.78 (s, 2H), 2.05 (s, 3H), 1.20 (s, 12H), 0.83 (s, 3H), 0.37 (s, 3H). LC-MS (ESI): m/z: calcd: 555.3330; found: $555.3300[\mathrm{M}-\mathrm{Cl}]^{+}$. 
Collections of quantum yield data and calculated $\Delta \mathrm{E}$

Table S1. Quantum yields of meso-phenyl substituted BODIPY dyes in polar solvents.

No.

Molecular structure

B1

B2<smiles></smiles>

0.83

0.56

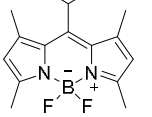

B3

$$
\text { - }{ }_{\mathrm{NH}}
$$

B4

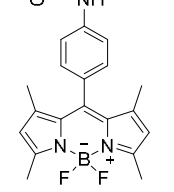

0.98

0.01

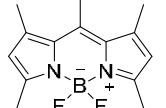

B5

B6

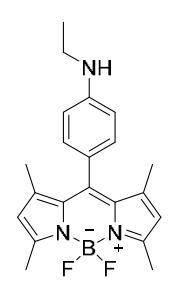

B7

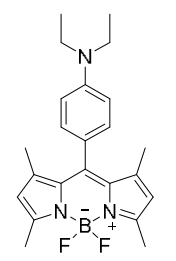

$<0.001$

$<0.01$

0.55

Water

Methanol

Methanol

Water

Water

Water

Reference

8

9

Ethanol 9

10

10

11

12

13 
B9

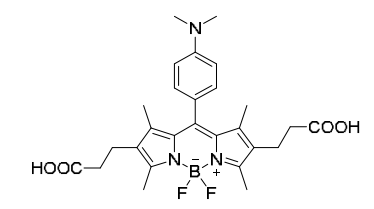

B10

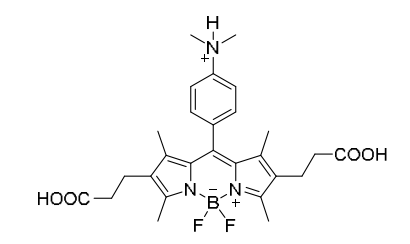

B11

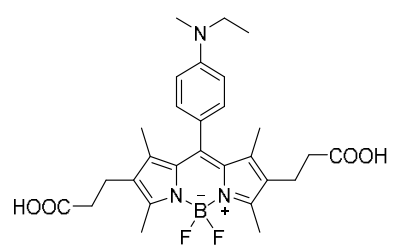

$<0.01$

0.56

Water

13

0.56
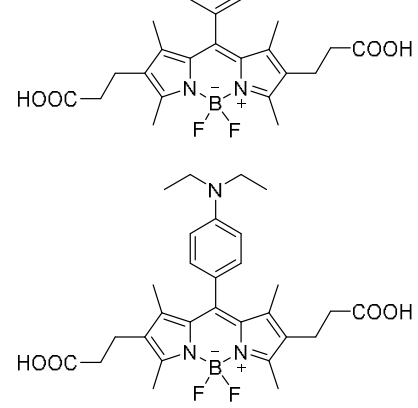

B13

$<0.01$

Water

Water

13

B12

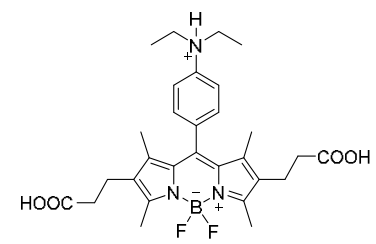

B15<smiles>Cc1c(CCC(=O)O)c(C)n(-c2ccccc2)c1-c1c(C)c(CCC(=O)O)c(C)n1P(F)F</smiles>

0.56

0.001

13

13

Water

Water

13

Water

B16

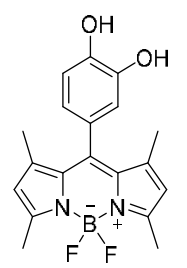


B17

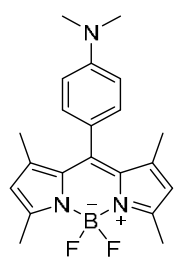

B18

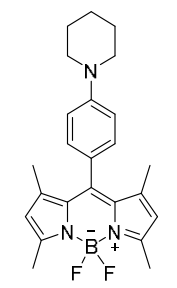

B19

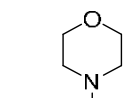

B20
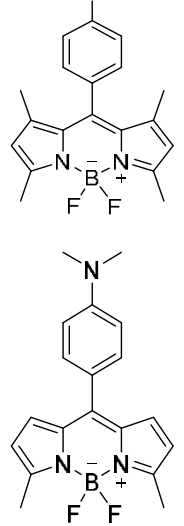

B21

B22

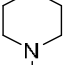

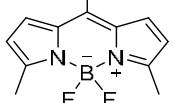<smiles>C1CCOC1</smiles>

B23
0.019

0.046

0.073

0.037

0.038

Methanol

Methanol

15

15

Methanol

0.008

Methanol

15

0.915

Methanol

16 


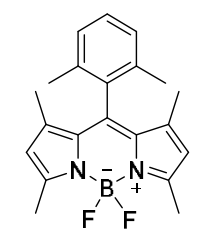

B25

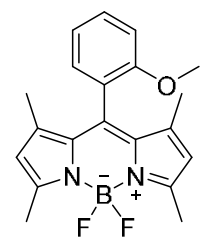

0.950

0.964

B26

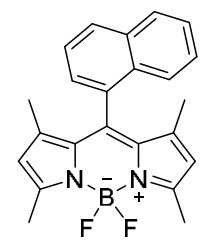

B27

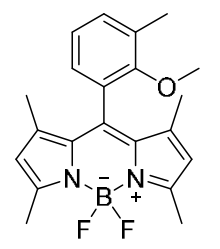

0.893

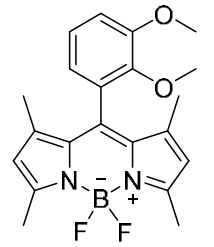

B29

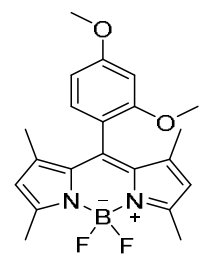

B30

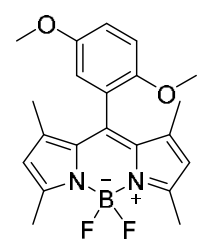

0.053

0.007

0.942

B31
Methanol

16

Methanol 16

Methanol 16

Methanol

16

$0.949 \quad$ Methanol 16

Methanol

16

Methanol

16

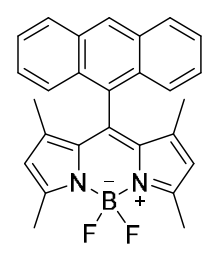


B32

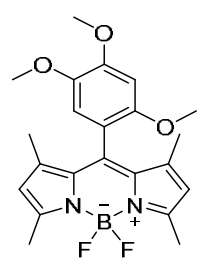

$<0.001$

Methanol

B33

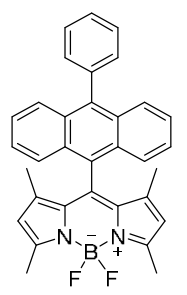

$<0.001$

Methanol

16

B34

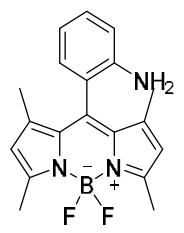

$<0.001$

Methanol

16

B35

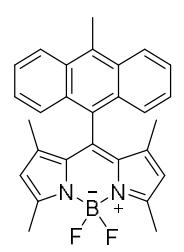

$<0.001$

Methanol

16

B36

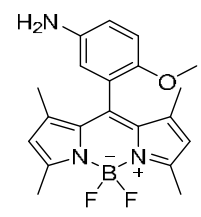

$<0.001$

Methanol

16

B37

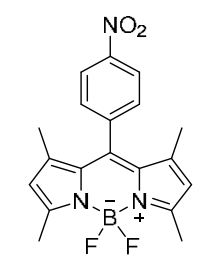

0.004

Methanol

17

B38

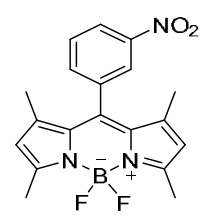

0.004

Methanol

17

B39

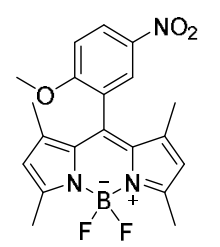

0.023

Methanol

17

B40

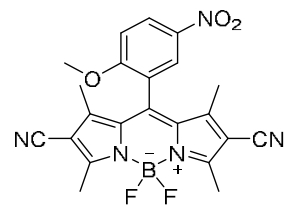

0.529

Methanol

17 
B41

B42<smiles>COc1ccc2c(c1)OC1C(C)=C(C#N)C(C)=C1C21c2c(C)c(C)c(C)n2P(F)n2c(C)c(C#N)c(C)c21</smiles>

B43<smiles>Oc1ccc(C2=C3C=CC(C3)N2P2(F)=CC=C2)cc1</smiles>

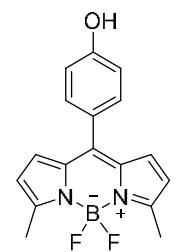

0.28

Methanol

B45

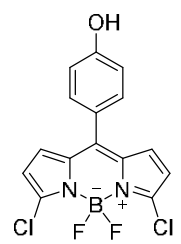

B46

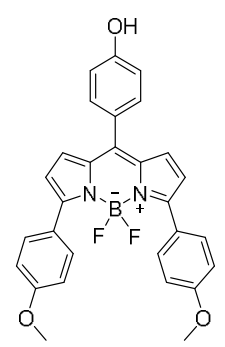<smiles>Cc1ccc2n1C(c1ccc(O)c(Cl)c1)c1ccc(C)n1[Y5]2(F)F</smiles>

B48

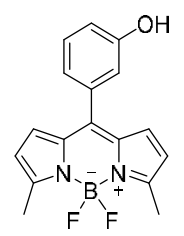

0.19

Methanol 


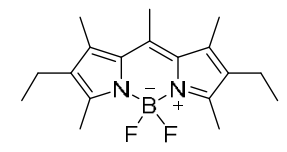

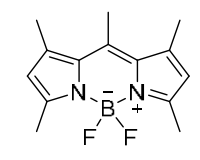

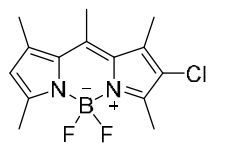

B52

B53<smiles>CC1=C(C)N(C)[P-](F)(F)n2c(C)c(Cl)c(C)c21</smiles><smiles>CCc1c(C)c2n(c1C)[P](F)(F)n1c(CC)c(C)c(CO)c1C2C</smiles>

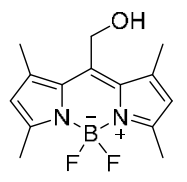

B55

B56

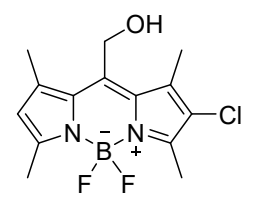

B57<smiles>Cc1c(Cl)c(C)n2c1-c1c(CO)c3c(C)c(Cl)c(C)n3n1[B-](C)(F)B2F</smiles>

B58

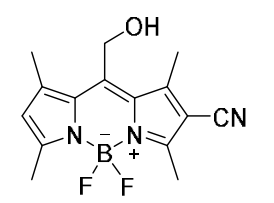

B59

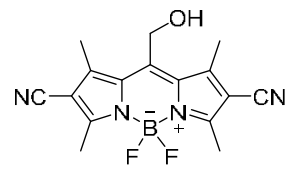

0.81

0.85

0.98

Acetonitrile

19

19

Acetonitrile

19

Acetonitrile

19

Acetonitrile

19

Acetonitrile

19

Acetonitrile

19

Acetonitrile

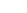

0.87

Acetonitrile

19

1

Acetonitrile

19 
B60

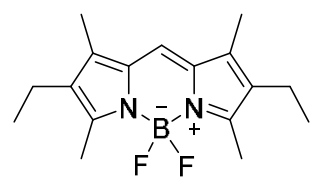

B61

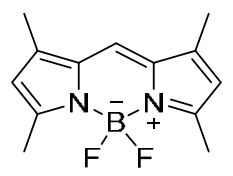

B62

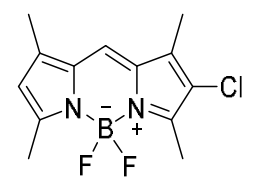

B63<smiles></smiles>

B64

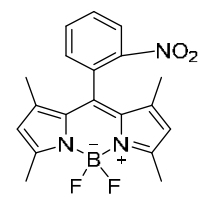

0.027

0.03

B65

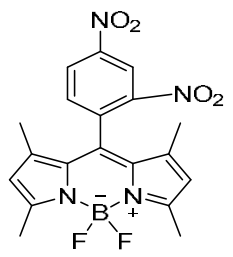

B66

B67
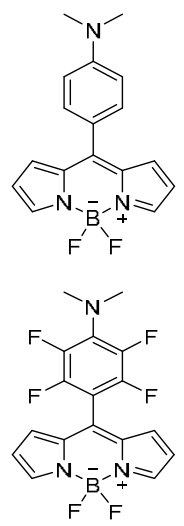

0.003

0.026

0.78

0.0002

B68
Acetonitrile

19

Acetonitrile

19

Methanol

20

Methanol

21

Methanol 20

Methanol

21

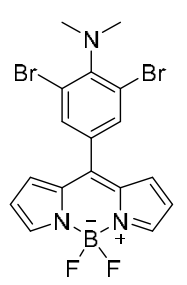



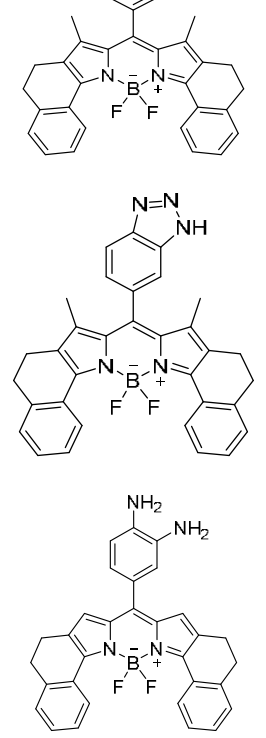

B73

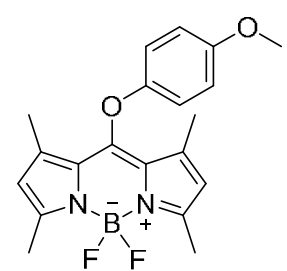<smiles></smiles>

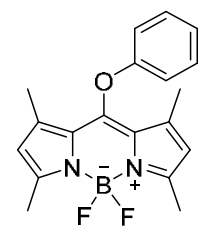

0.96

0.001

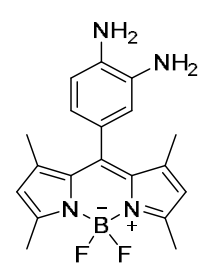


B77

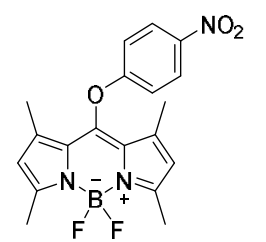

B78

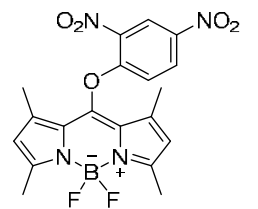

B79

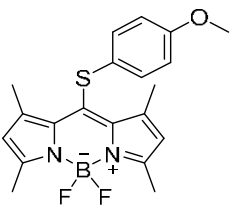

B80

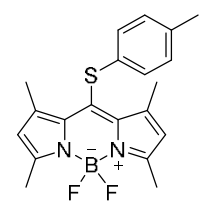

B81

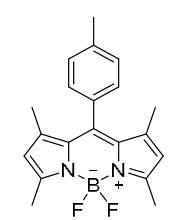

0.559

0.007

0.40

0.0012

0

0

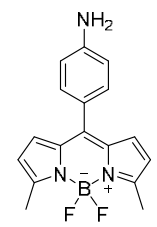

B83
Acetonitrile

24

Acetonitrile

Acetonitrile

24

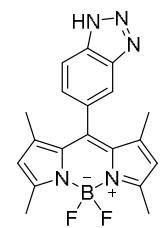


Table S2. Calculated $\triangle E$ values of BODIPY dyes at $\omega B$ 97XD/Def2SVP level.

\begin{tabular}{|c|c|c|c|c|c|}
\hline Molecule & $\Delta \mathrm{E}(\mathrm{eV})$ & Molecule & $\Delta \mathrm{E}(\mathrm{eV})$ & Molecule & $\Delta \mathrm{E}(\mathrm{eV})$ \\
\hline B1 & 0.878 & B29 & 0.685 & B57 & 1.287 \\
\hline B2 & 0.837 & B30 & 0.302 & B58 & 1.382 \\
\hline B3 & 0.841 & B31 & 0.012 & B59 & 1.351 \\
\hline B4 & 0.195 & B32 & 0.518 & B60 & 1.348 \\
\hline B5 & 0.038 & B33 & -0.007 & B61 & 1.539 \\
\hline B6 & -0.247 & B34 & 0.318 & B62 & 1.279 \\
\hline B7 & 0.306 & B35 & -0.105 & B63 & 1.279 \\
\hline B8 & 1.342 & B36 & -0.164 & B64 & 0.41 \\
\hline B9 & -0.005 & B37 & 0.266 & B65 & 0.273 \\
\hline B10 & 1.35 & B38 & 0.281 & B66 & -0.589 \\
\hline B11 & -0.014 & B39 & 0.428 & B67 & -0.273 \\
\hline B12 & 1.345 & B40 & 0.896 & B68 & -0.232 \\
\hline B13 & -0.146 & B41 & 0.252 & B69 & 0.17 \\
\hline B14 & 1.341 & B42 & 0.622 & B70 & 0.17 \\
\hline B15 & 1.332 & B43 & 0.417 & B71 & 0.329 \\
\hline B16 & 0.539 & B44 & 0.707 & B72 & 0.08 \\
\hline B17 & -0.342 & B45 & 0.478 & B73 & 0.382 \\
\hline B18 & -0.23 & B46 & 0.752 & B74 & 0.837 \\
\hline B19 & -0.147 & B47 & 0.746 & B75 & 1.11 \\
\hline B20 & -0.35 & B48 & 0.731 & B76 & 1.365 \\
\hline B21 & -0.412 & B49 & 0.242 & B77 & -0.197 \\
\hline B22 & -0.223 & B50 & 1.32 & B78 & 0.438 \\
\hline B23 & 1.338 & B51 & 1.436 & B79 & 0.253 \\
\hline B24 & 1.236 & B52 & 1.222 & B80 & 0.525 \\
\hline B25 & 0.854 & B53 & 1.238 & B81 & 1.338 \\
\hline B26 & 0.694 & B54 & 1.355 & B82 & 0.012 \\
\hline B27 & 1.269 & B55 & 1.484 & B83 & 1.326 \\
\hline B28 & 0.818 & B56 & 1.283 & & \\
\hline
\end{tabular}


Table S3. Reference codes and dihedral angles returned from the structural search of tetramethyl BODIPY dyes in CCDC.

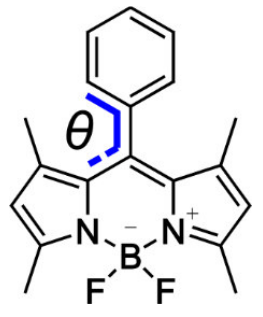

\begin{tabular}{|c|c|c|c|c|c|c|c|}
\hline Refcode & $\theta\left(^{\circ}\right)$ & Refcode & $\theta\left(^{\circ}\right)$ & Refcode & $\theta\left(^{\circ}\right)$ & Refcode & $\theta\left({ }^{\circ}\right)$ \\
\hline BINSUP & 84.36 & CIVYAK & 92.57 & YILLEN & 79.31 & AQEQIY & 75.60 \\
\hline BINTAW & 85.66 & COCHEJ04 & 88.19 & ADOPOA & 92.30 & ASETOI & 80.60 \\
\hline CIMMUI05 & 90.86 & FITCAP & 85.31 & ADOPUG & 95.24 & ASETOI01 & 81.20 \\
\hline CIVNAZ & 93.30 & FITCET & 81.50 & ADOSEU & 89.72 & ASETOI02 & 103.84 \\
\hline CIVNED & 109.05 & FITCET & 87.47 & ADOSEU & 95.28 & ASETOI02 & 76.06 \\
\hline CIVNIH & 104.72 & FITCIX & 90.71 & ADOSEU & 82.95 & ASETOI03 & 80.96 \\
\hline CIVNON & 96.77 & FITCIX & 96.81 & ADOSIY & 98.65 & ASOGEU & 84.10 \\
\hline CIVNON & 76.10 & FIVNIK & 86.57 & ADOSIY & 87.04 & ASOGEU & 85.35 \\
\hline CIVPEF & 91.54 & FIVNIK & 93.03 & ADOSIY & 91.02 & ATOXIS & 96.27 \\
\hline CIVPEF & 91.42 & GIVSUC & 92.22 & ADOSOE & 88.54 & ATOXOY & 81.55 \\
\hline CIVPIJ & 81.66 & GIVSUC & 89.46 & ADOSOE & 90.03 & ATOXOY & 88.46 \\
\hline CIVPIJ & 88.45 & GIVSUC & 90.32 & ADOSOE & 88.99 & ATOYAL & 88.96 \\
\hline CIVPIJ & 94.03 & OYULIF01 & 89.88 & AFIKEH & 78.67 & ATOYEP & 90.45 \\
\hline CIVPIJ & 82.51 & SIQWEX & 90.69 & AFIKEH01 & 86.38 & ATOYIT & 89.98 \\
\hline CIVPIJ & 85.87 & SIQWIB & 69.39 & AGEPAF & 87.94 & AYOWUH & 78.82 \\
\hline CIVPIJ & 86.33 & SIQWIB & 96.07 & AHIFOO & 91.73 & BAWHIS & 77.63 \\
\hline CIVPIJ & 93.89 & SIQWOH & 102.59 & AHILEK & 92.40 & BAWHIS & 76.68 \\
\hline CIVPIJ & 94.47 & SIQWOH & 79.33 & AKEJOR & 84.77 & BAWHUE & 96.85 \\
\hline CIVYAK & 102.44 & SIQWUN & 72.35 & APUNEG & 99.47 & BEHZEV & 107.52 \\
\hline CIVYAK & 92.57 & WOLFOU02 & 78.31 & APUNIK & 100.57 & BEHZEV01 & 73.45 \\
\hline BELYEZ & 77.41 & CAJPAG & 81.92 & DEMMEQ & 78.89 & EBACAM & 96.04 \\
\hline BELYEZ & 99.14 & CAJPEK & 71.54 & DEMMIU & 92.46 & EFEVER & 97.55 \\
\hline
\end{tabular}




\begin{tabular}{|c|c|c|c|c|c|c|c|}
\hline BELYID & 103.23 & CIHBIG & 88.78 & DEMMUG & 92.60 & EFEVIV & 102.09 \\
\hline BELYOJ & 81.97 & CIMMUI & 97.49 & DESTAZ & 88.66 & EFEVIV & 100.86 \\
\hline BIBQEK & 84.52 & CIMMUI & 86.60 & DESTED & 89.69 & EGEJAD & 90.15 \\
\hline BIBQEK & 87.20 & CIMMUI01 & 92.81 & DESTED & 89.87 & EGEMOU & 82.74 \\
\hline BIBQIO & 82.29 & CIMMUI02 & 97.90 & DETZUA & 88.77 & EGEMUA & 82.64 \\
\hline BIBQIO & 86.34 & CIMMUI03 & 90.50 & DONDER & 108.20 & EGENAH & 102.85 \\
\hline BIBQOU & 106.59 & CIMMUI04 & 90.05 & DONDER & 87.61 & EGICII & 97.34 \\
\hline BIBQOU & 111.30 & COCHEJ & 84.32 & DONDIV & 83.55 & EGICOO & 88.16 \\
\hline BITSEE & 100.54 & COCHEJ01 & 86.41 & DONDUH & 88.31 & EGIDAB & 88.44 \\
\hline BUVMUC & 90.90 & COCHEJ02 & 95.45 & DUBPIB & 84.04 & EGIDEF & 89.65 \\
\hline BUVMUC & 97.07 & COCHEJ03 & 82.19 & DUBPOH & 85.52 & EGIDEF & 85.84 \\
\hline CADXOW & 90.07 & COCKUC & 108.35 & DUBPOH & 86.28 & EGIDIJ & 87.44 \\
\hline CADXOW & 103.04 & COSYIU & 88.75 & DUBPOH & 93.81 & EGIDIJ & 79.88 \\
\hline CADXOW & 92.19 & DACZIS & 99.59 & DUBPOH & 94.52 & EGIYAV & 93.54 \\
\hline CADXOW & 100.68 & DACZIS & 72.40 & DUHGUK & 79.18 & EJEHUY & 99.27 \\
\hline CAHZES & 84.36 & DEMMEQ & 92.22 & DUMSOV & 97.30 & EJEJAG & 92.93 \\
\hline CAJNUY & 101.16 & DEMMEQ & 82.91 & EBABUF & 101.63 & EKUJEA & 85.20 \\
\hline CAJPAG & 64.74 & DEMMEQ & 89.47 & EBACAM & 81.84 & EKUJIE & 110.23 \\
\hline BELYEZ & 77.41 & CAJPAG & 81.92 & DEMMEQ & 78.89 & EBACAM & 96.04 \\
\hline EQIJUK & 75.63 & EQORUY & 82.83 & FASSIE & 88.32 & FIQPEC & 81.45 \\
\hline EQIKEV & 103.23 & EQORUY & 93.19 & FASSIE & 93.20 & FUJJAW & 100.09 \\
\hline EQIKEV & 97.99 & EQOSIN & 95.62 & FENWEC & 88.56 & FUJJAW & 68.97 \\
\hline EQOROS & 89.84 & EYOLEK & 89.91 & FIDKEK & 78.91 & GOZBEE & 88.87 \\
\hline EQOROS01 & 96.83 & FAQNOD & 100.78 & FIDLIP & 90.73 & GOZBEE & 91.59 \\
\hline EQORUY & 81.22 & FAQNOD & 77.39 & FIDLOV & 98.45 & GUFDOC & 80.99 \\
\hline EQORUY & 84.35 & FAQNUJ & 86.60 & FIDLUB & 82.87 & GUFDOC & 77.48 \\
\hline EQORUY & 77.72 & FAQPAR & 77.64 & FIDMAI & 98.08 & GUFZEO & 82.85 \\
\hline GUFZUE & 97.60 & GUFZIS & 95.47 & GUFZOY & 96.41 & GUFZUE & 79.62 \\
\hline
\end{tabular}




\begin{tabular}{|c|c|c|c|c|c|c|c|}
\hline GUGBAN & 96.40 & HUJHAX & 108.85 & JUCBAM & 102.16 & KEYBIC & 66.44 \\
\hline GUGBAN & 93.23 & HUJHAX01 & 73.19 & JUCBEQ & 73.47 & KEYFUS & 110.20 \\
\hline GUQJEJ & 95.54 & ICIBON & 93.77 & JUGLAA & 103.25 & KEYFUS & 65.95 \\
\hline GUQJIN & 85.53 & IDIHUB & 80.24 & JUGLAA & 95.27 & KOGFUJ & 89.76 \\
\hline GUQJOT & 92.04 & IDIWAV & 89.33 & JUGLEE & 91.81 & KUSPUK & 91.23 \\
\hline GUQJOT & 71.81 & ILUFAY & 79.60 & JUGLII & 80.26 & LABXOE & 64.20 \\
\hline GUZKES & 74.44 & IMOYIU & 91.34 & KABZAR & 74.36 & LABXUK & 72.64 \\
\hline GUZKIW & 87.15 & INOHIE & 84.49 & KABZAR & 90.52 & LABYAR & 74.83 \\
\hline GUZKIW & 95.98 & IQUQUH & 100.06 & KABZEV & 87.60 & LABYEV & 79.58 \\
\hline HACFAV & 79.84 & IQUQUH & 88.53 & KABZIZ & 80.26 & LACLIN & 78.89 \\
\hline HAQXEF & 78.94 & IQUQUH & 81.47 & KABZIZ & 90.49 & LASQUT & 84.31 \\
\hline HAQXEF & 91.14 & JIMKOG & 86.07 & KACNIO & 74.10 & LASQUT & 78.58 \\
\hline HAQXEF & 88.77 & JIMKOG & 98.54 & KACNOU & 91.75 & LASRAA & 98.88 \\
\hline HAQXEF & 74.99 & JIZLUA & 92.29 & KAKNAN & 88.87 & LASRAA & 111.96 \\
\hline HAQXEF & 94.35 & JIZLUA & 99.32 & KAKNAN & 91.13 & LEWDEZ & 90.09 \\
\hline HAQXEF & 84.40 & JIZMAH & 100.36 & KAKNER & 82.96 & LIXHUX & 91.06 \\
\hline HESJAT & 93.30 & JONJON & 91.27 & KAKNER & 90.18 & LIZSOD & 97.26 \\
\hline HEZQEK & 84.38 & JOWHAG & 74.53 & KAKNIV & 106.29 & LIZSOD & 81.05 \\
\hline HUCLEY & 87.78 & JOWHEK & 89.20 & KARSON & 101.95 & LIZSOD & 92.33 \\
\hline HUJGUQ & 61.64 & JOWHIO & 93.27 & KARSUT & 109.71 & LIZSOD & 87.58 \\
\hline LONHUT & 74.83 & MELMAU & 77.41 & NABLEK & 90.85 & NIPKOO & 97.84 \\
\hline LUJWUJ & 90.57 & MENQAA & 95.89 & NABLEK & 95.38 & NIPKUU & 78.36 \\
\hline LUJXAQ & 87.53 & MENQII & 98.51 & NADTOE & 85.87 & NIPKUU & 98.92 \\
\hline LUVKIY & 96.34 & MEZKEJ & 76.94 & NADTUK & 96.95 & ONOYOH & 92.08 \\
\hline MECQOD & 90.50 & MIFJET & 99.99 & NAZTOY & 80.81 & OQERAE & 104.00 \\
\hline MEKJOC & 99.86 & MIGSEB & 73.04 & NEJLEW & 79.17 & OQERAE01 & 83.90 \\
\hline MELLAT & 81.21 & MIRNAE & 90.59 & NEJLIA & 83.39 & OQUTEB & 86.02 \\
\hline MELLEX & 89.09 & MOCZEK & 90.72 & NEKSUU & 81.75 & OSIPOX & 98.08 \\
\hline
\end{tabular}




\begin{tabular}{|c|c|c|c|c|c|c|c|}
\hline & & & & & & & \\
\hline MELLIB & 86.90 & MOCZIO & 78.70 & NEKSUU & 107.80 & OVAYER & 71.11 \\
\hline MELLIB & 103.26 & NABJEI & 69.46 & NEKTAB & 100.77 & OXOQID & 98.92 \\
\hline MELMAU & 95.98 & NABJOS & 112.09 & NEKTAB & 97.84 & OYULIF & 90.66 \\
\hline OYULIF & 90.03 & PIFJUK & 100.39 & QAFBIK & 76.05 & RASLEF & 103.26 \\
\hline PAKCAI & 81.42 & PIFMAV & 100.07 & QAFBUW & 91.05 & RASLIJ & 90.88 \\
\hline PAKCEM & 74.89 & PIFMAV01 & 85.17 & QAHSEA & 110.21 & REGTUU & 89.48 \\
\hline PARJEZ & 86.20 & PIFMAV02 & 85.72 & QAHSOK & 90.21 & REGVAC & 81.03 \\
\hline PARJID & 99.21 & PIFMAV03 & 97.09 & QEFHUH & 75.72 & RERLOS & 95.75 \\
\hline PATJIG & 90.03 & PIJROS & 92.35 & QEFHUH & 82.33 & RETLUX & 107.98 \\
\hline PATJOM & 83.55 & PIXJIQ & 82.72 & QONBOL & 121.58 & RETLUX & 73.69 \\
\hline PATJUS & 91.53 & PIYDAE & 92.50 & QONBUR & 65.54 & ROZGIY & 101.85 \\
\hline PATJUS & 93.38 & PIYDAE & 96.69 & QONCAY & 119.73 & ROZJAT & 73.01 \\
\hline PATKAZ & 96.83 & POBWAG & 96.67 & QUSWUY & 68.44 & ROZJAT & 95.13 \\
\hline PATKED & 92.26 & POBWAG & 75.34 & RADHIQ & 76.56 & ROZJEX & 70.25 \\
\hline PATKIH & 85.31 & POCZAJ & 124.29 & RAGVOL & 111.52 & RUSBAK & 80.50 \\
\hline PAVRAG & 102.48 & POCZEN & 66.76 & RAGVUR & 63.47 & RUSBEO & 86.70 \\
\hline PAVVUG & 88.50 & POWPOI & 104.35 & RAGWIG & 67.88 & RUSBEO & 102.23 \\
\hline PERJEE & 94.89 & PUTBUD & 87.50 & RAGWIG01 & 66.17 & RUSBEO & 98.31 \\
\hline PERKAB & 91.36 & PUTBUD & 109.84 & RAGWOM & 92.83 & SAFXOO & 100.05 \\
\hline PESLOR & 93.79 & PUTCAK & 105.59 & RASKUU & 92.94 & SAFXOO & 108.63 \\
\hline PEWDUT & 93.07 & PUTCAK & 103.11 & RASLAB & 76.92 & SAFX0001 & 96.38 \\
\hline PEWFAB & 74.17 & QAFBEG & 75.69 & RASLEF & 88.70 & SAFX0001 & 68.58 \\
\hline SAFX0002 & 83.72 & SAVFEC & 72.01 & SEGTEG & 102.09 & SOQLOA & 90.17 \\
\hline SAJCUD & 105.15 & SAVFIG & 75.86 & SEGTIK & 79.24 & SUJJOY & 89.05 \\
\hline SALFUJ & 112.57 & SAVFOM & 100.04 & SEJDUH & 96.21 & SUJJUE & 99.96 \\
\hline SALGAQ & 105.20 & SAVFUS & 105.63 & SESLIO & 107.55 & SUQFIU & 85.07 \\
\hline SALGEU & 105.57 & SAVFUS & 66.22 & SICSIJ & 104.33 & TAWXUN & 104.80 \\
\hline SALHAQ & 92.08 & SAVFUS & 86.25 & SIQZOJ & 87.58 & TEDKIY & 70.92 \\
\hline
\end{tabular}




\begin{tabular}{|c|c|c|c|c|c|c|c|}
\hline SANROQ & 77.70 & SAXDIH & 77.31 & SOFBAS & 76.66 & TEDKIY & 87.98 \\
\hline SANROQ & 100.26 & SAXDIH & 87.26 & SOQLEQ & 107.37 & TEDKOE & 110.71 \\
\hline SAVFEC & 76.65 & SAXMAI & 85.27 & SOQLIU & 80.50 & TEDKOE & 96.00 \\
\hline TEGPIF & 74.30 & UNIFIG & 87.34 & WAKTOT & 108.66 & WUVTUE & 88.70 \\
\hline TEQMIN & 77.93 & UPAYIU & 91.90 & WICLAX & 99.68 & XAWREV & 88.66 \\
\hline TERFOO & 92.82 & USILOY & 90.04 & WICLAX & 80.13 & XAWROF & 93.55 \\
\hline TIBJOF & 88.02 & USILUE & 100.21 & WICLAX & 80.41 & XAWRUL & 84.86 \\
\hline TIBJUL & 90.91 & USILUE & 106.98 & WICLAX & 82.31 & XAWSAS & 87.76 \\
\hline TIBKAS & 85.94 & USIMAL & 95.47 & WIHHUR & 98.20 & XAWSAS & 90.95 \\
\hline TIBKEW & 90.36 & USIMAL & 103.68 & WIYZEL & 86.13 & XAWSEW & 97.10 \\
\hline TIBKIA & 78.29 & UTOVAB & 96.70 & WIYZIP & 84.01 & XAWSEW & 97.07 \\
\hline TIBKIA & 90.08 & UYIWIK & 98.35 & WOLFOU & 86.01 & XEBZOW & 84.94 \\
\hline TIBKIA & 75.43 & UZAVIC & 93.49 & WOLFOU01 & 92.86 & XEBZOW & 91.77 \\
\hline TIBKIA & 86.24 & VAHRIH & 75.41 & WORSUT & 81.55 & XIJJIL & 87.72 \\
\hline TINTIV & 87.98 & VESRIV & 84.78 & WORTAA & 87.57 & XIMCIF & 88.35 \\
\hline UCIQII & 57.41 & VICVEK & 79.31 & WUFLAM & 84.12 & XISMIX & 75.30 \\
\hline UJOPOA & 84.70 & VIDTIN & 91.50 & WULMIA & 101.97 & XISMIX & 67.28 \\
\hline UJOPOA & 90.63 & VIDTOT & 77.20 & WULMOG & 82.70 & XOGBIF & 74.00 \\
\hline UKEJOL & 83.58 & VONWIF & 67.08 & WUMXEJ & 82.77 & XOGDON & 85.13 \\
\hline UNEWUH & 67.13 & VONWUR & 116.86 & WUMXIN & 83.71 & XOGFEF & 85.47 \\
\hline UNEWUH & 73.27 & VOXLOL & 78.89 & WURNAZ & 110.85 & XOGFIJ & 87.70 \\
\hline UNIFIG & 101.51 & WAKTIN & 91.54 & WUVTIS & 84.55 & XOGFIJ & 94.68 \\
\hline UNIFIG & 76.19 & WAKTIN & 91.76 & WUVTOY & 94.65 & XOGFIJ & 97.06 \\
\hline XOXGAU & 107.75 & YENCEB & 101.68 & YIWFIV & 80.18 & YURTOW & 86.26 \\
\hline XOXGAU & 74.88 & YENCIF & 95.10 & YIWFIV & 97.56 & ZARDUT & 101.43 \\
\hline XOXGEY & 86.73 & YENRIV & 104.76 & YOBWIX & 78.55 & ZEFBET & 77.48 \\
\hline XUFNAO & 96.24 & YENRIV & 83.05 & YONKAO & 81.67 & ZEFBIX & 93.53 \\
\hline XUFNES & 100.47 & YENRIV & 79.47 & YONKES & 85.92 & ZOFKAI & 79.56 \\
\hline
\end{tabular}




\begin{tabular}{|c|c|c|c|c|c|c|c|}
\hline XUFNES01 & 78.28 & YEWCAF & 95.01 & YUCHUB & 84.81 & ZOFKEM & 75.00 \\
\hline XUKLUM & 103.42 & YEXJAN & 79.61 & YUCJAJ & 78.00 & ZOFKIQ & 86.30 \\
\hline XUXFIH & 94.59 & YEXJER & 92.44 & YUKGES & 86.58 & ZUCGIP & 90.33 \\
\hline YAMXIW & 72.64 & YIVJEU & 93.37 & YURTEM & 86.26 & ZULBEP & 90.71 \\
\hline YEHYOC & 111.68 & YIVJIY & 83.47 & YURTIQ & 79.98 & ZULBOZ & 75.81 \\
\hline CIVXUD & 75.81 & JIZRUI & 99.26 & JOGFOD & 74.88 & RIYYAC & 77.86 \\
\hline DOGVED & 89.52 & JocBOV & 89.37 & LOCNUP & 87.63 & WOFQUG & 88.90 \\
\hline JIZRUI & 80.51 & JOCHER & 74.06 & NIZFEK & 63.97 & WOFRAN & 72.79 \\
\hline YIZMUS & 91.82 & & & & & & \\
\hline
\end{tabular}


Table S4. Molecular structures, calcuated $\Delta \mathrm{E}(\mathrm{eV})$, UV-Vis absorption peaks $\left(\lambda_{\mathrm{abs}} ; \mathrm{nm}\right)$, emission peaks $\left(\lambda_{\text {emi; }}\right.$; $\mathrm{nm}$ ), and quantum yields (QY) of meso-phenyl substituted BODIPY dyes in various solvents.

No.

B1<smiles>Oc1ccccc1</smiles>

B2<smiles></smiles>

0.872

0.829

B3<smiles>COc1ccccc1</smiles>

B4<smiles>C=C(C)C1=C(C(=C)C)n2c(C)cc(C)[n+]2B(F)n2c(C)cc(C)c21</smiles>

0.815

B5<smiles></smiles>

0.203

0.034

B6<smiles></smiles>

$-0.239$

solvent
Hexane
Dichloromethane
ethyl acetate
Ethanol
acetonitrile
Methanol
DMSO
Hexane

Dichloromethane ethyl acetate

Ethanol

acetonitrile

Methanol

DMSO

Hexane

Dichloromethane

ethyl acetate

Ethanol

acetonitrile

Methanol

DMSO

Hexane

Dichloromethane

ethyl acetate

Ethanol

acetonitrile

Methanol

DMSO

Hexane

Dichloromethane

ethyl acetate

Ethanol

acetonitrile

Methanol

DMSO

Hexane

Dichloromethane

ethyl acetate

Ethanol

acetonitrile

Methanol

DMSO
Aabs

501

500

498

498

497

497

501

505

501

498

499

497

498

501

501

501

498

499

497

501

501

500

500

498

498

496

497

496

500

500

498

498

496

497

500

500

499

498

498

496

496

500 $\lambda$ emi

QY

$509 \quad 0.390$

$509 \quad 0.497$

$507 \quad 0.467$

$507 \quad 0.467$

$506 \quad 0.392$

$506 \quad 0.405$

511

0.182

509

510

508

508

506

507

511

512

512

508

508

508

508

512

509

510

509

509

508

509

514

508

512

511

508

507

510

514

508

510

508

509

507

507

511
0.454

0.558

0.485

0.484

0.463

0.444

0.551

0.325

0.504

0.441

0.490

0.397

0.398

0.634

0.404

0.102

0.003

0.001

0.001

0.002

0.002

0.314

0.001

0.001

0.001

0.001

0.001

0.001

0.279

0.010

0.009

0.004

0.008

0.008

0.010 
Table S5. Quantum yields of meso-phenyl substituted O/Si-rhodamine dyes in polar solvents.

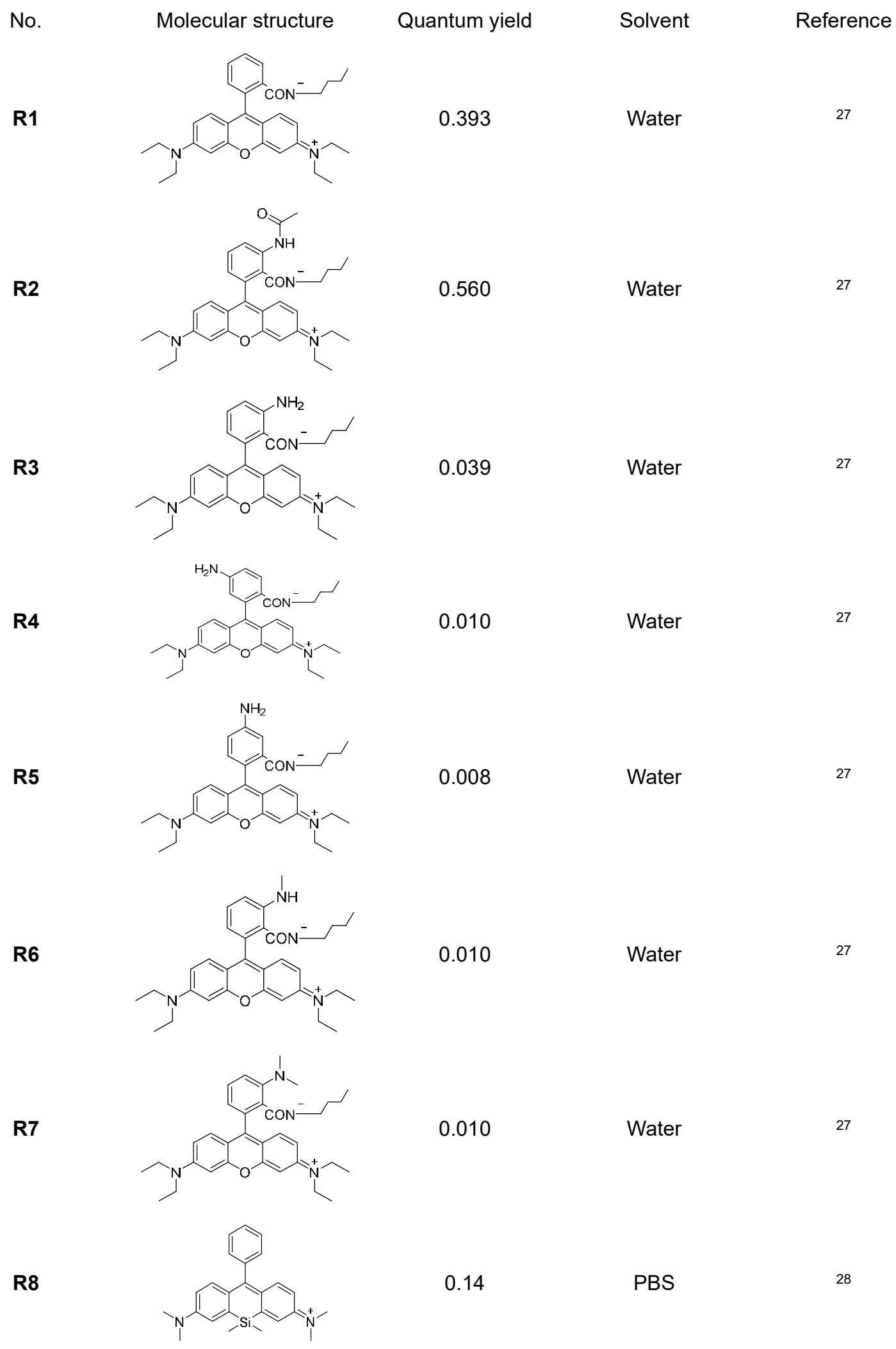


R9

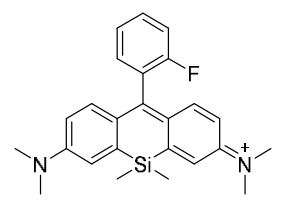

0.19

PBS

28

R10<smiles>CN(C)c1ccc2c(c1)[Si](C)(C)C1=CC(=[N+](C)C)C=CC1=C2c1ccccc1Cl</smiles>

0.30

PBS

28

R11<smiles>Cc1ccccc1C1=C2C=CC(=[N+](C)C)C=C2[Si](C)(C)c2cc(N(C)C)ccc21</smiles>

0.31

PBS

28

R12

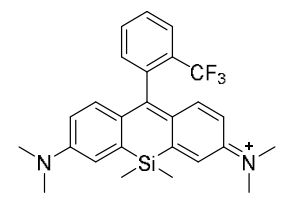

0.33

PBS

28

R13

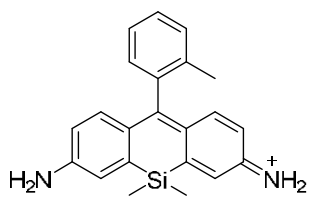

0.38

PBS

28

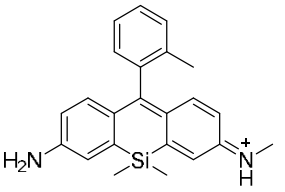

0.31

PBS

28

R14

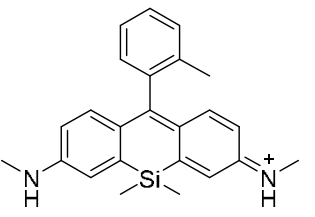

0.43

PBS

28

R15

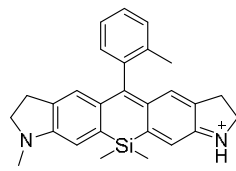

0.13

PBS

28

R16

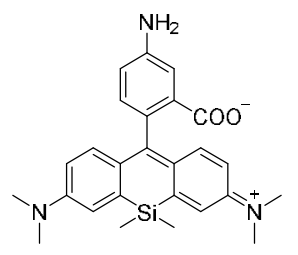

0.018

Water

29

R18

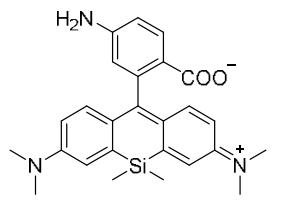

0.016

Water

29 
R19
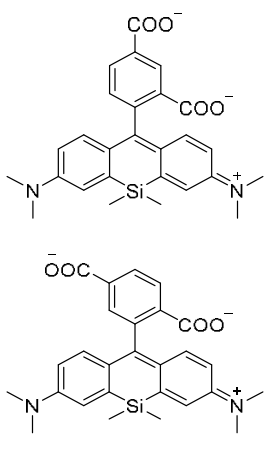

R21

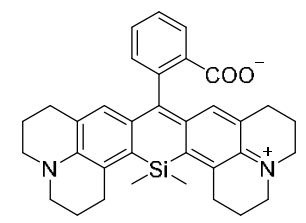

R22

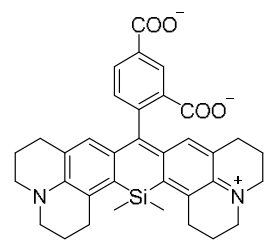

R23

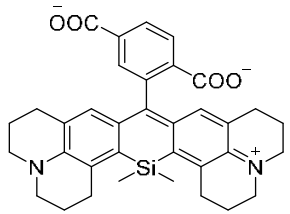

R24

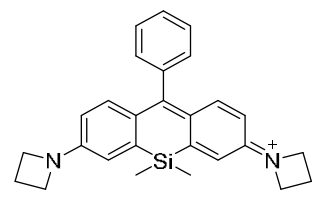

R25

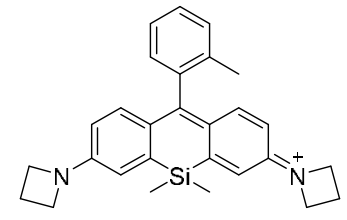

R26

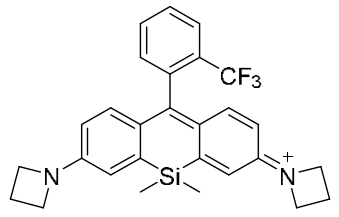

R27
0.98

0.79

0.56

0.59

0.83

0.19

0.59

0.54

0.53
Water

Water

Water

Water

29

29

29

29

Water 29

PBS 30

PBS 30

PBS 30

Ethanol 31 


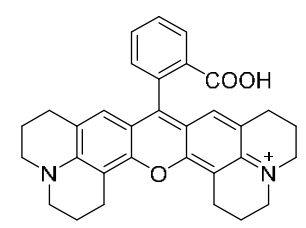

Table S6. Quantum yields of meso-phenyl substituted fluorescein dyes in polar solvents.

No.

Molecular structure

F1

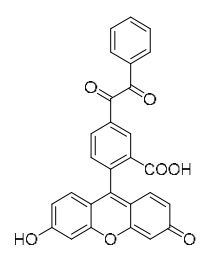

F2

F3

F4

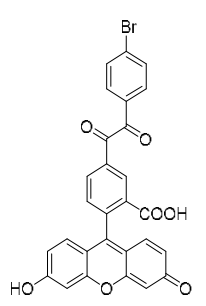

0.022

0.026

0.060

0.004

PBS

Water

0.30

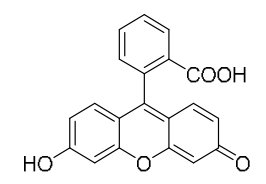

F7

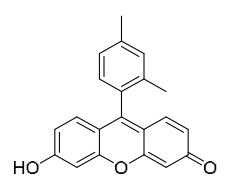

Solvent

Reference

PBS

32

PBS

32

PBS

32

PBS

32
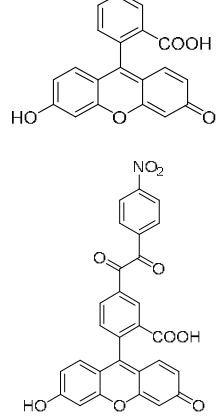

F6

0.307

Water

33 
F8

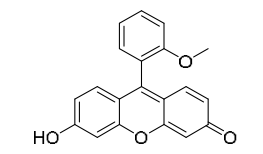

F9

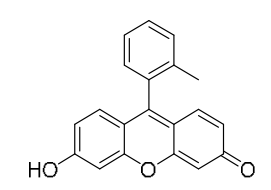

F10<smiles>C=CCC1CCCC1CC</smiles><smiles>O=c1ccc2cc3ccc(O)cc3oc-2c1</smiles><smiles></smiles>

F12

F11

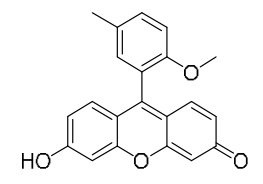

F13

F14
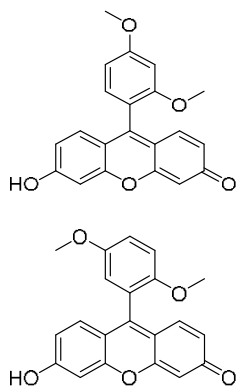

F15

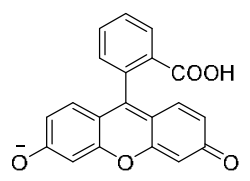

F16

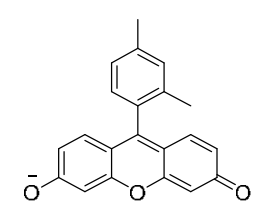

F17<smiles>COc1ccccc1-c1c2ccc(=O)cc-2oc2cc(O)ccc12</smiles>

F18
0.076

0.319

0.319

0.010

0.004

0.001

0.004

0.850

Water

0.865

0.860

0.847

Water

Water

Water

Water
33

33

Water 33

33

33

Water 33

Water 33

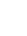

33

Water 33

Water 33

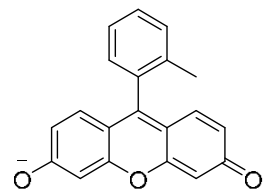


F19

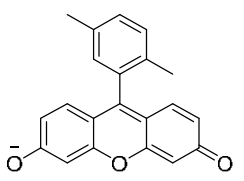

F20

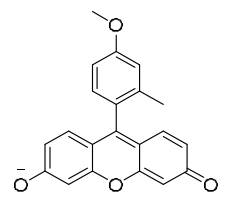

F21

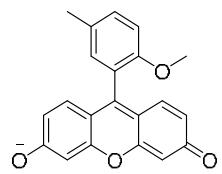

F22

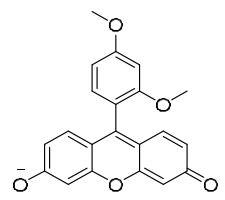

F23
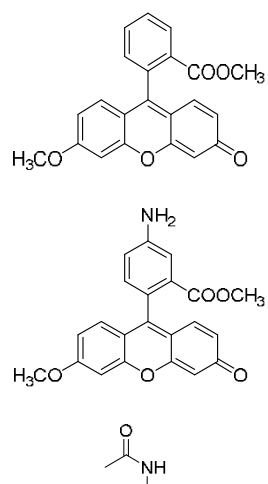

F25

F24
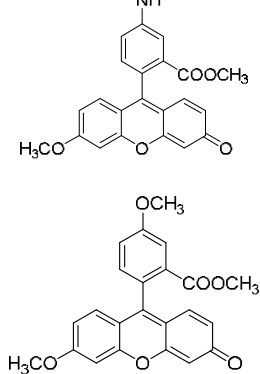

F27

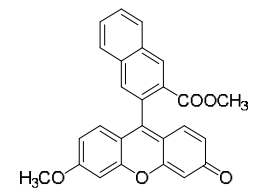

F28

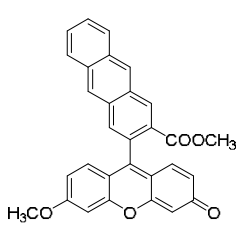

0.887

0.840

0.500

Water

33

0.200

Water

33

0.270

PBS

34

0.008

PBS

34

0.210

PBS

34

0.270

PBS

34

0.260

PBS

34

0.010

PBS

33

33
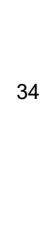

34 


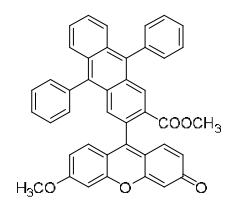


Table S7. Molecular structures, calculated $\Delta \mathrm{E}(\mathrm{eV})$, UV-Vis absorption peaks $\left(\lambda_{\mathrm{abs}} ; \mathrm{nm}\right)$, emission peaks $\left(\lambda_{\text {emi; }}\right.$; $\mathrm{nm}$ ), and quantum yields (QY) of M1, M2, and M3 in various solvents.

\begin{tabular}{|c|c|c|c|c|c|c|}
\hline No. & Structure & $\Delta \mathrm{E}$ & solvent & $\lambda_{a b s}$ & $\lambda_{\mathrm{emi}}$ & QY \\
\hline \multirow{6}{*}{ M1 } & & \multirow{6}{*}{1.024} & Dichloromethane & 561 & 580 & 0.226 \\
\hline & & & ethyl acetate & 560 & 580 & 0.191 \\
\hline & & & Ethanol & 561 & 582 & 0.205 \\
\hline & & & acetonitrile & 559 & 581 & 0.209 \\
\hline & & & Methanol & 562 & 580 & 0.206 \\
\hline & & & DMSO & 569 & 591 & 0.199 \\
\hline \multirow{6}{*}{ M2 } & & \multirow{6}{*}{0.522} & Dichloromethane & 561 & 578 & 0.0884 \\
\hline & & & ethyl acetate & 560 & 582 & 0.00592 \\
\hline & & & Ethanol & 560 & 578 & 0.00188 \\
\hline & & & acetonitrile & 558 & 578 & 0.00147 \\
\hline & & & Methanol & 559 & 576 & 0.00126 \\
\hline & & & DMSO & 565 & 588 & 0.000981 \\
\hline \multirow{6}{*}{ M3 } & & \multirow{6}{*}{1.012} & Dichloromethane & 566 & 585 & 0.206 \\
\hline & & & ethyl acetate & 561 & 581 & 0.170 \\
\hline & & & Ethanol & 562 & 581 & 0.197 \\
\hline & & & acetonitrile & 561 & 583 & 0.190 \\
\hline & & & Methanol & 561 & 584 & 0.202 \\
\hline & & & DMSO & 568 & 591 & 0.198 \\
\hline
\end{tabular}


Calculated frontier molecular orbitals and corresponding $\Delta \mathrm{E}$ of meso-phenyl substituted BODIPY-based compounds<smiles></smiles>
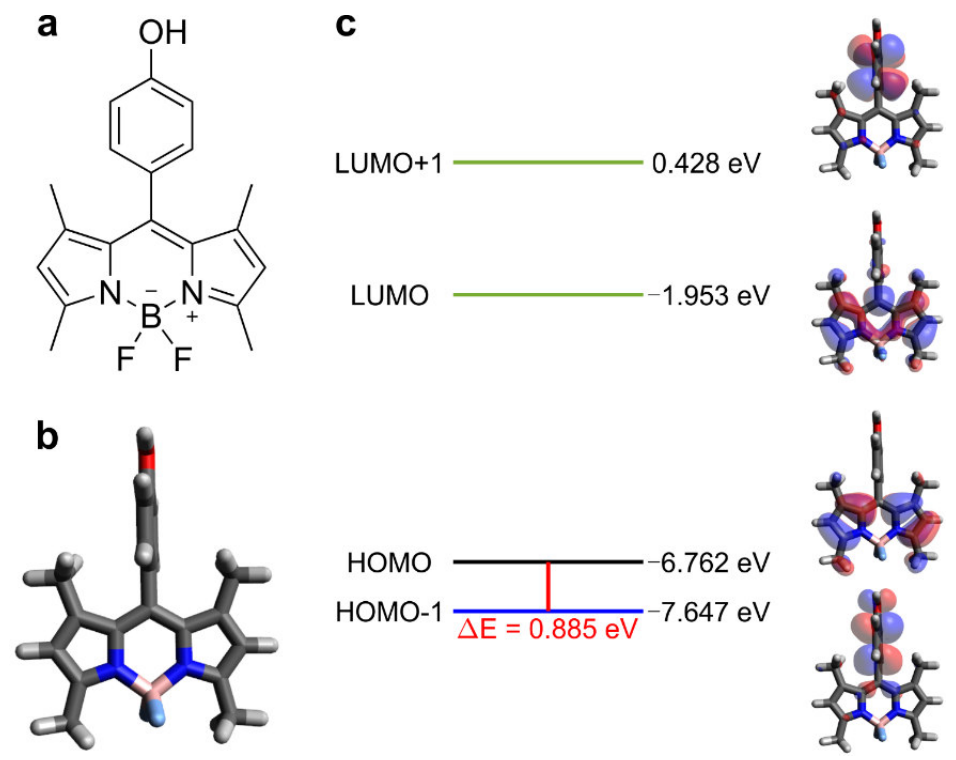

Figure S3. a) Molecular structure, b) optimized structure, c) frontier molecular orbitals and corresponding energy levels, and calculated $\Delta \mathrm{E}$ of $\mathrm{B} 1$ in the ground state in ethanol at M062X/Def2SVP level.
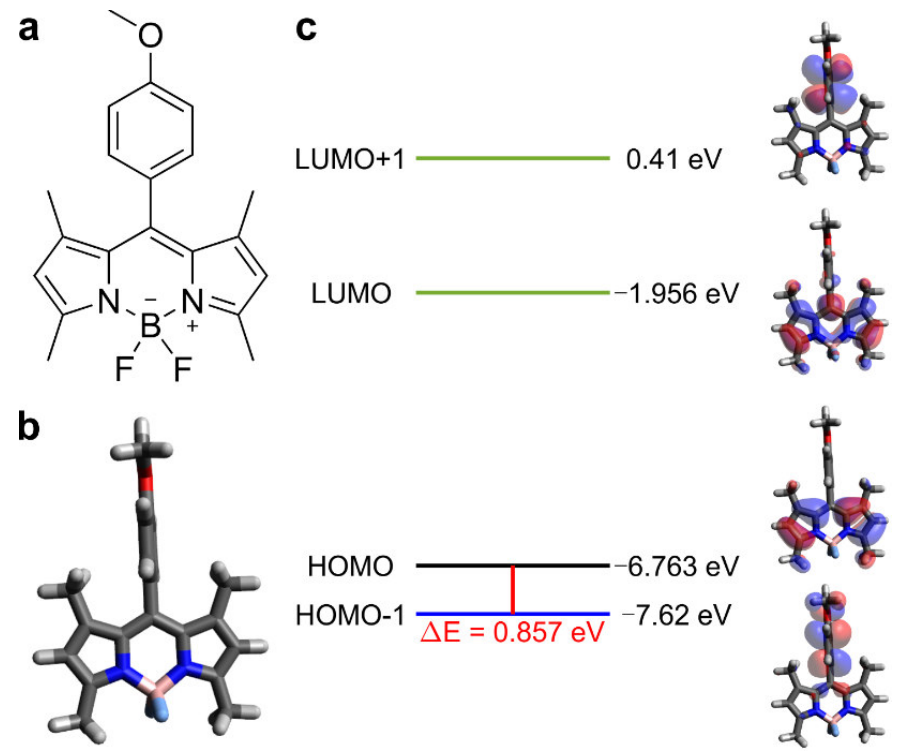

Figure S4. a) Molecular structure, b) optimized structure, c) frontier molecular orbitals and corresponding energy levels, and calculated $\Delta \mathrm{E}$ of $\mathrm{B2}$ in the ground state in ethanol at M062X/Def2SVP level. 
a

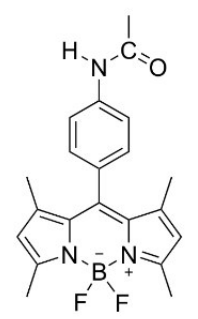

b

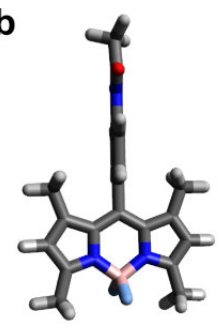

C
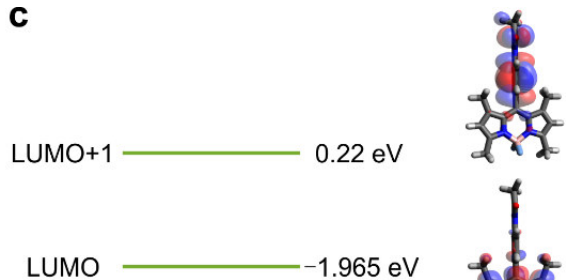

HOMO $-6.767 \mathrm{eV}$

HOMO-1 $\frac{}{\Delta \mathrm{E}=0.808 \mathrm{eV}}-7.575 \mathrm{eV}$

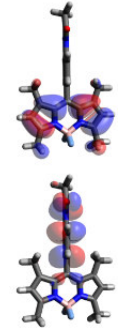

Figure S5. a) Molecular structure, b) optimized structure, c) frontier molecular orbitals and corresponding energy levels, and calculated $\Delta \mathrm{E}$ of B3 in the ground state in methanol at M062X/Def2SVP level.

a<smiles></smiles>

b

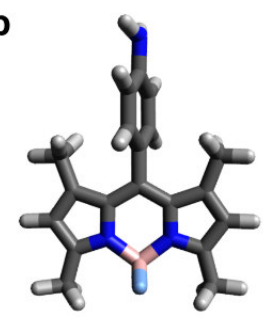

C

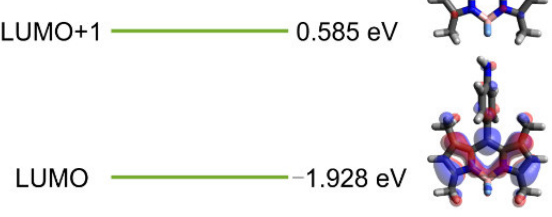
$\underset{\mathrm{HOMO}-1}{\mathrm{HOMO}} \frac{1}{\Delta \mathrm{E}=0.164 \mathrm{eV}}-6.911 \mathrm{eV}$

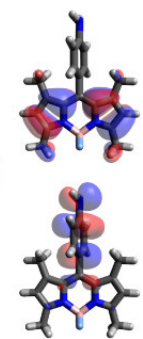

Figure S6. a) Molecular structure, b) optimized structure, c) frontier molecular orbitals and corresponding energy levels, and calculated $\Delta \mathrm{E}$ of B4 in the ground state in methanol at M062X/Def2SVP level. 
a

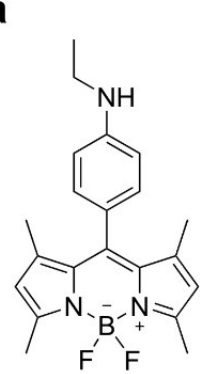

C

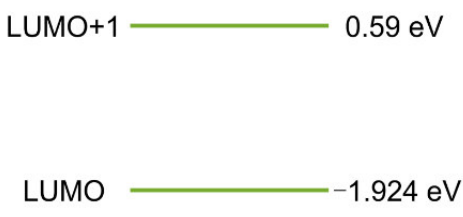

b

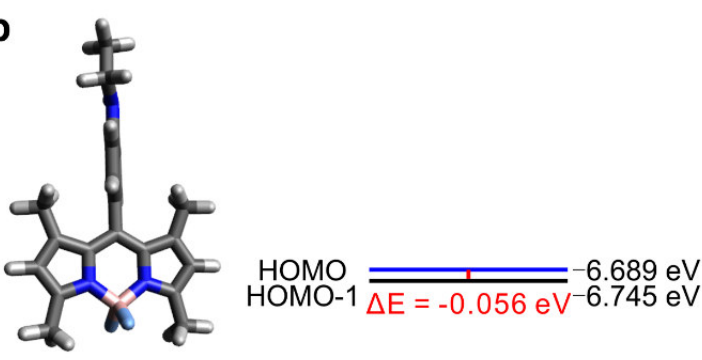

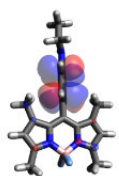
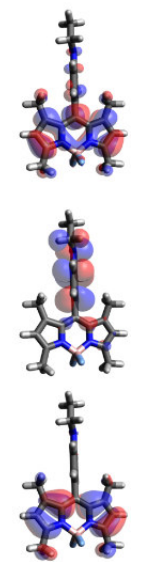

Figure S7. a) Molecular structure, b) optimized structure, c) frontier molecular orbitals and corresponding energy levels, and calculated $\Delta \mathrm{E}$ of B5 in the ground state in water at M062X/Def2SVP level.

a

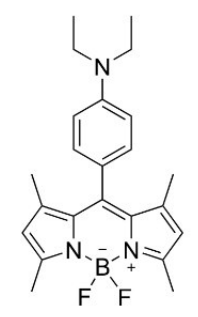

b

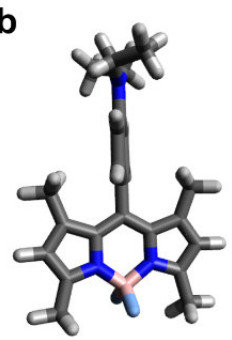

C
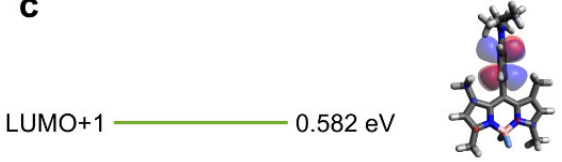

LUMO
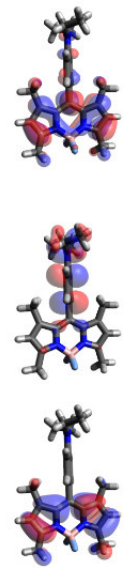

Figure S8. a) Molecular structure, b) optimized structure, c) frontier molecular orbitals and corresponding energy levels, and calculated $\Delta \mathrm{E}$ of B6 in the ground state in water at M062X/Def2SVP level. 
a
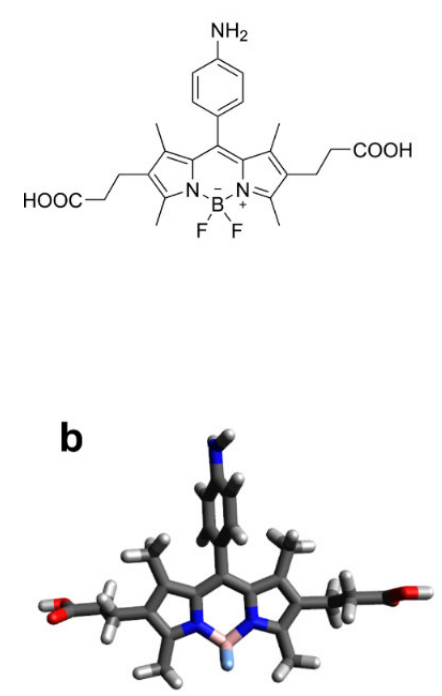

C

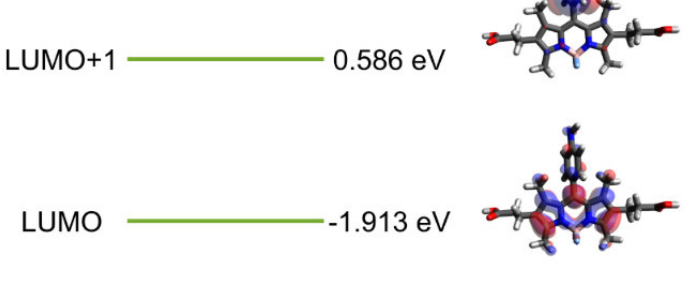

HOMO

HOMO-1 $\frac{1}{\Delta \mathrm{E}=0.273 \mathrm{eV}}-6.916 \mathrm{eV}$
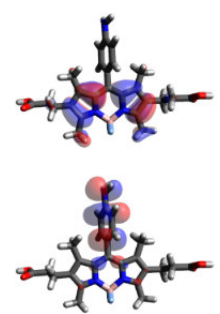

Figure S9. a) Molecular structure, b) optimized structure, c) frontier molecular orbitals and corresponding energy levels, and calculated $\Delta \mathrm{E}$ of B7 in the ground state in water at M062X/Def2SVP level.
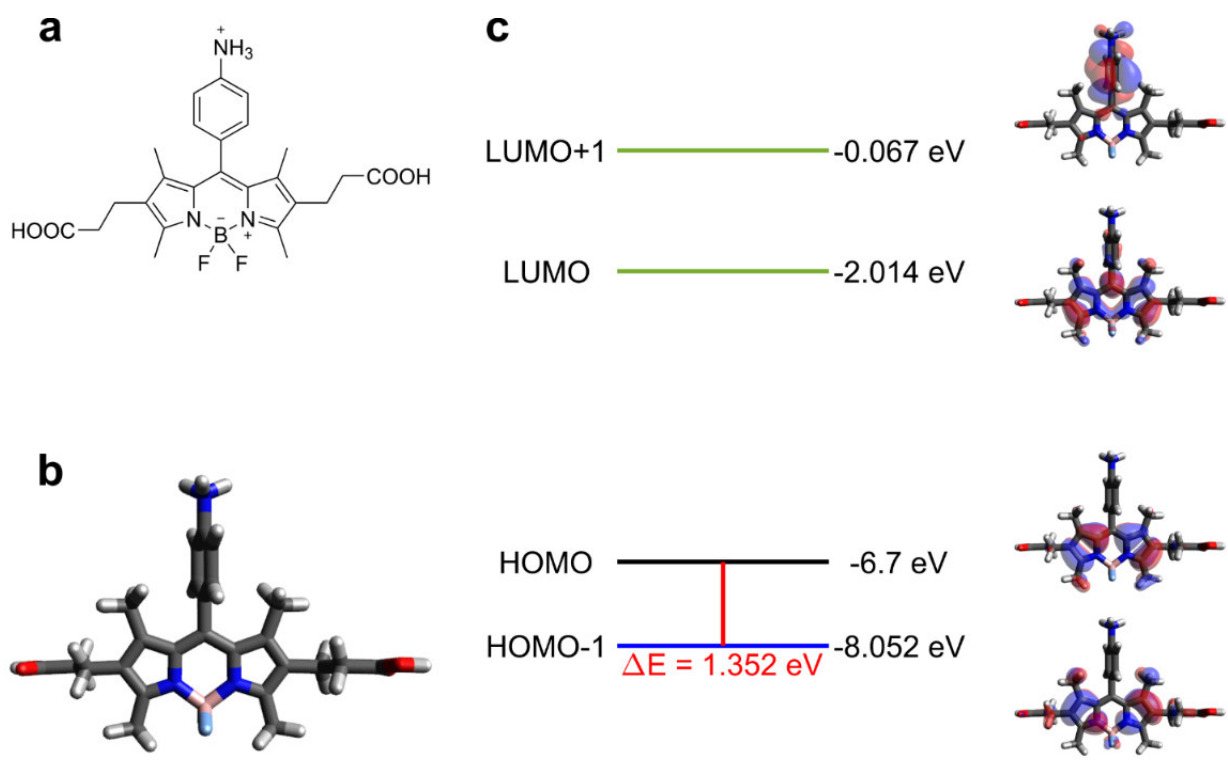

Figure S10. a) Molecular structure, b) optimized structure, c) frontier molecular orbitals and corresponding energy levels, and calculated $\Delta \mathrm{E}$ of B8 in the ground state in water at M062X/Def2SVP level. 

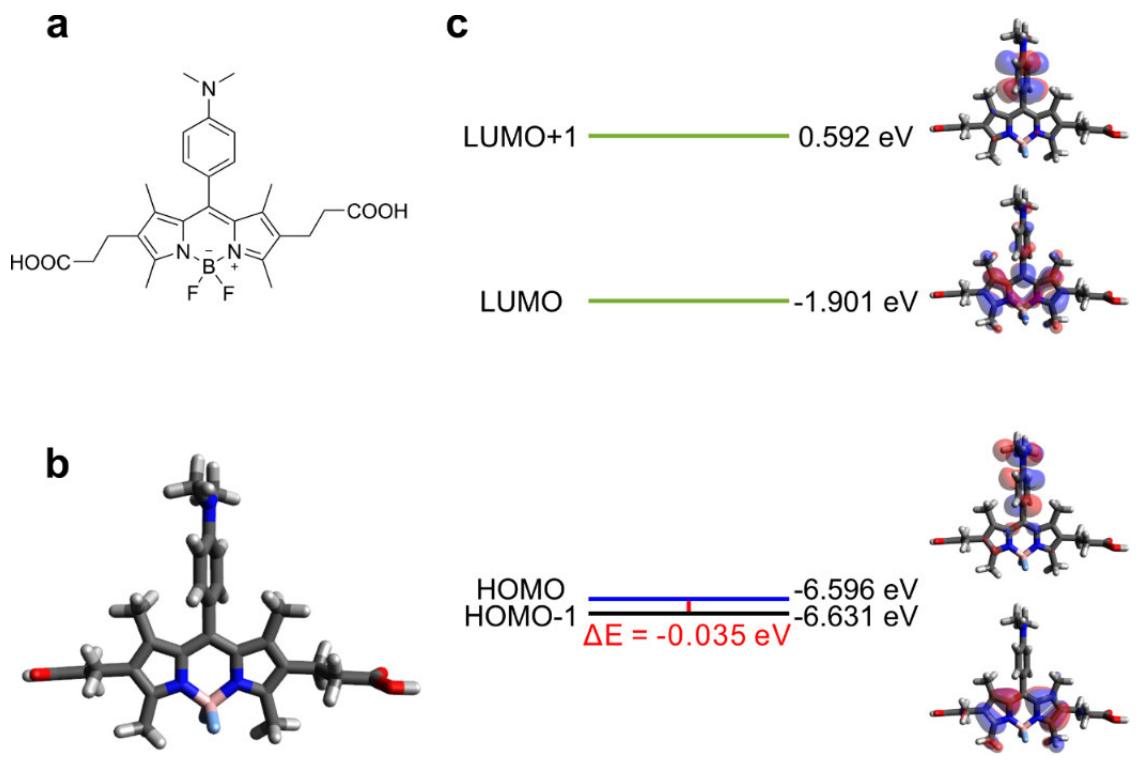

Figure S11. a) Molecular structure, b) optimized structure, c) frontier molecular orbitals and corresponding energy levels, and calculated $\triangle \mathrm{E}$ of $\mathrm{B} 9$ in the ground state in water at M062X/Def2SVP level.
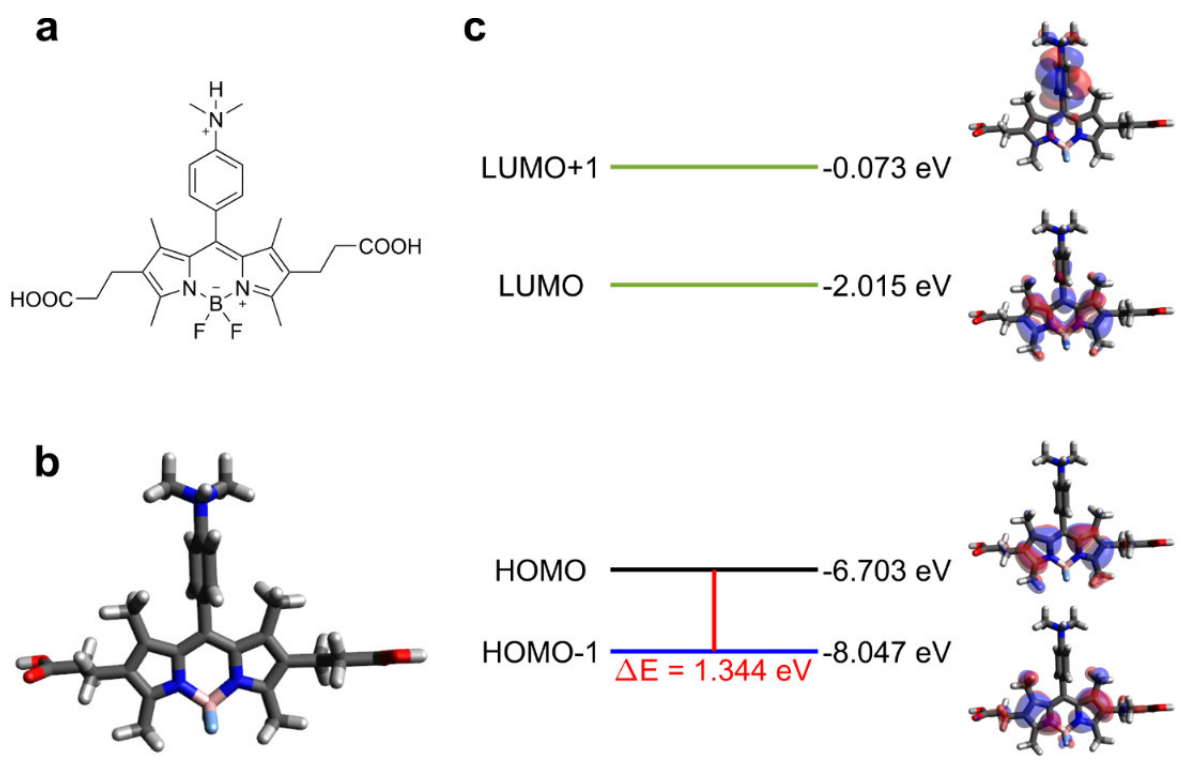

Figure S12. a) Molecular structure, b) optimized structure, c) frontier molecular orbitals and corresponding energy levels, and calculated $\Delta \mathrm{E}$ of B10 in the ground state in water at M062X/Def2SVP level. 
a

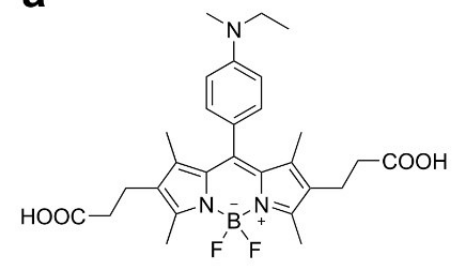

C

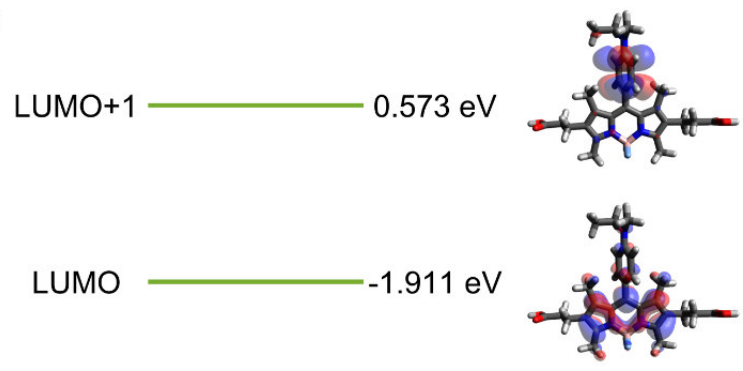

b

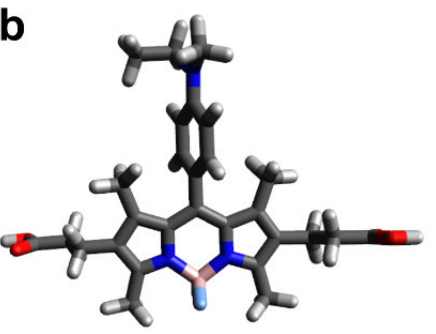

HOMO

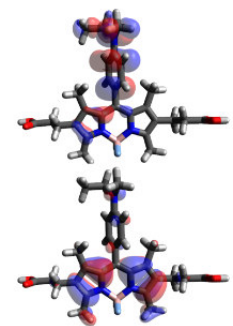
HOMO-1 $\overline{\frac{}{\Delta E=-0.013 e V}}-6.64 \mathrm{eV}$

Figure S13. a) Molecular structure, b) optimized structure, c) frontier molecular orbitals and corresponding energy levels, and calculated $\Delta \mathrm{E}$ of B11 in the ground state in water at M062X/Def2SVP level.

a

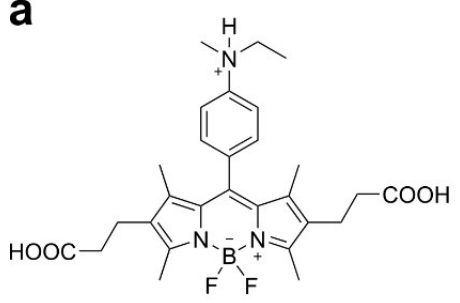

b

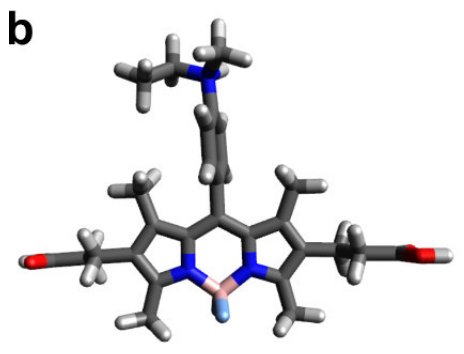

\section{C}

LUMO+1 $-0.059 \mathrm{eV}$

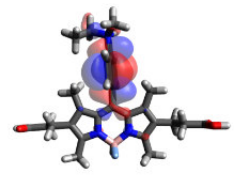

LUMO $-2.023 \mathrm{eV}$

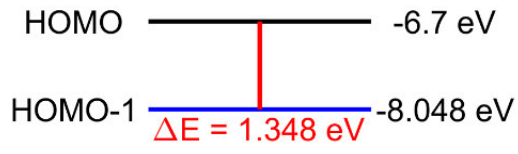

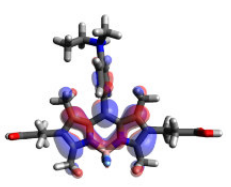
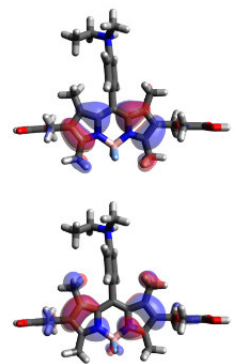

Figure S14. a) Molecular structure, b) optimized structure, c) frontier molecular orbitals and corresponding energy levels, and calculated $\Delta E$ of B12 in the ground state in water at M062X/Def2SVP level. 
a
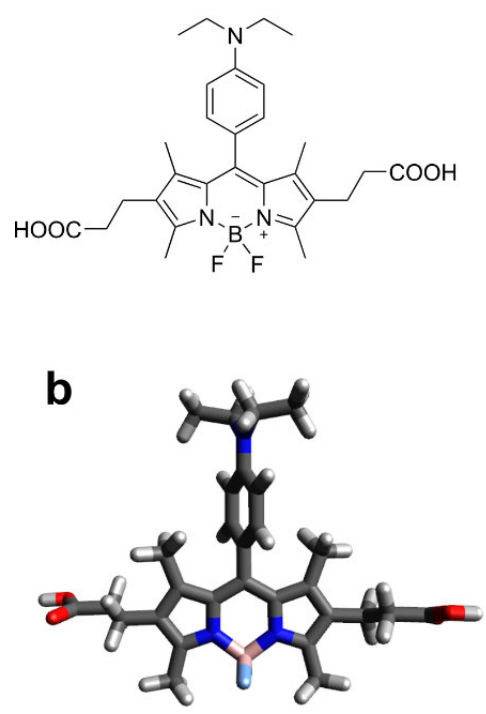

C

LUMO+1

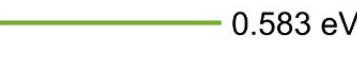

LUMO

$-1.906 \mathrm{eV}$
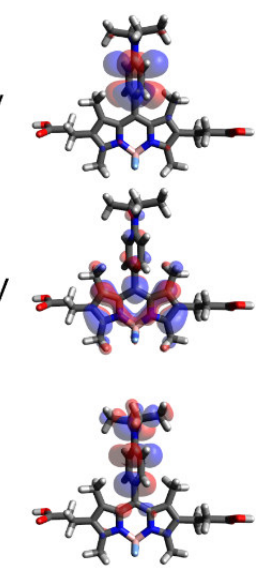

$\mathrm{HOMO}_{\mathrm{HOMO}}^{\mathrm{HOMO}} \overline{\overline{\Delta E=-0.165 \mathrm{eV}}}^{-6.4 .64 \mathrm{eV}}$

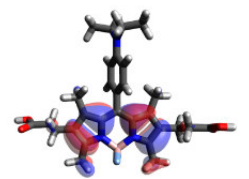

Figure S15. a) Molecular structure, b) optimized structure, c) frontier molecular orbitals and corresponding energy levels, and calculated $\Delta \mathrm{E}$ of B13 in the ground state in water at M062X/Def2SVP level.
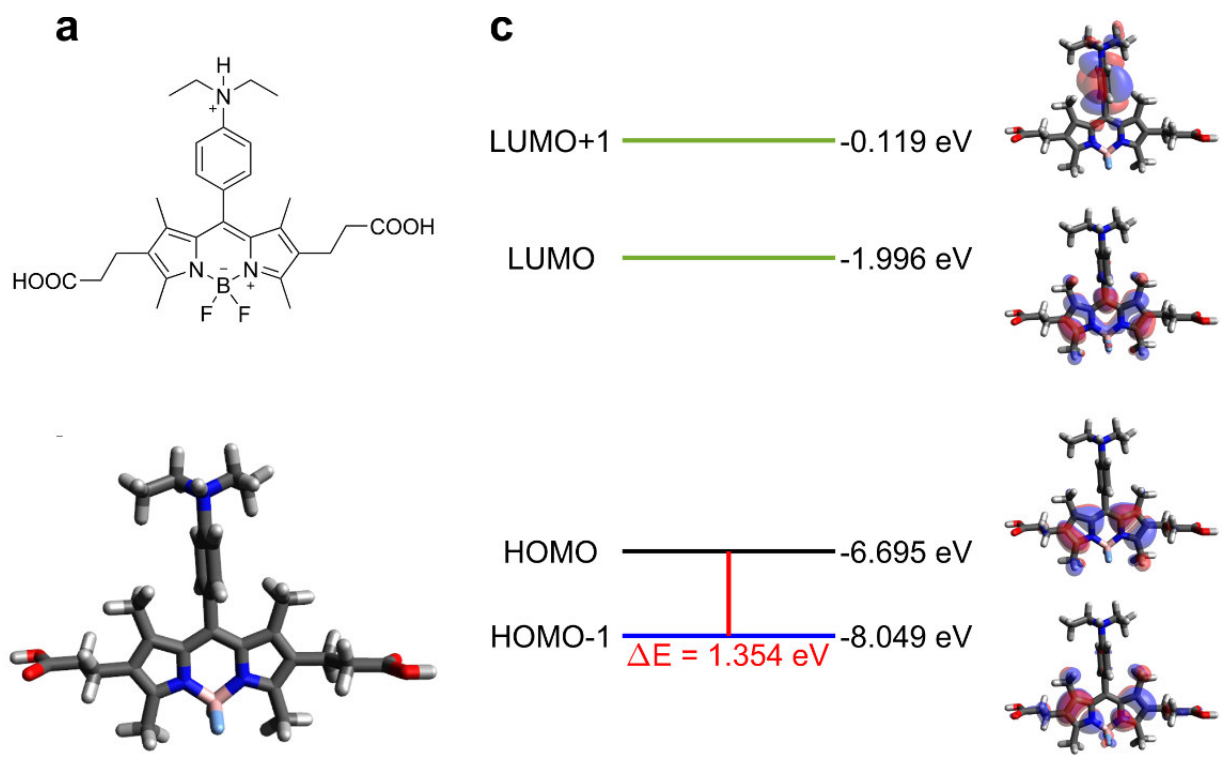

Figure S16. a) Molecular structure, b) optimized structure, c) frontier molecular orbitals and corresponding energy levels, and calculated $\Delta \mathrm{E}$ of B14 in the ground state in water at M062X/Def2SVP level. 
a

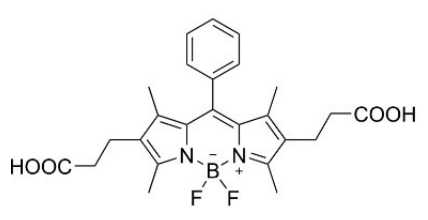

C

LUMO+1

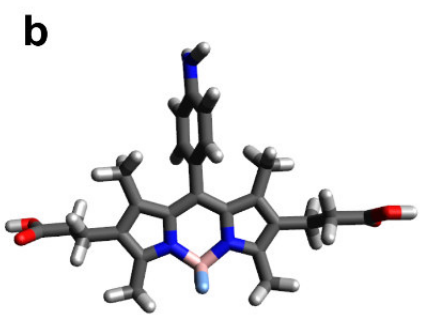

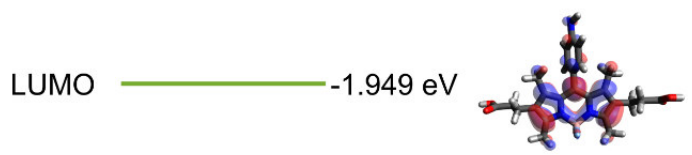

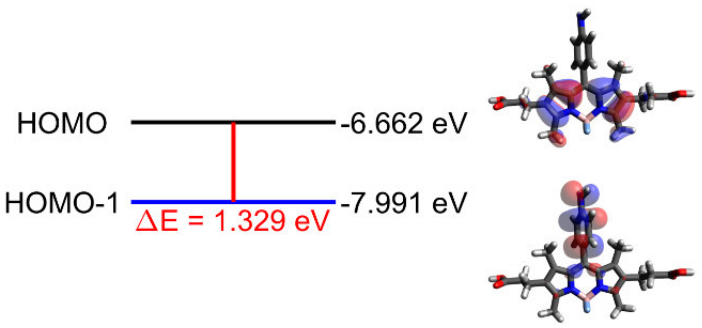

Figure S17. a) Molecular structure, b) optimized structure, c) frontier molecular orbitals and corresponding energy levels, and calculated $\Delta \mathrm{E}$ of B15 in the ground state in water at M062X/Def2SVP level.

a<smiles></smiles>

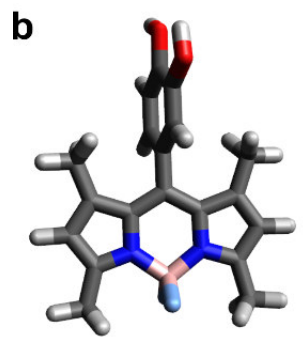

C

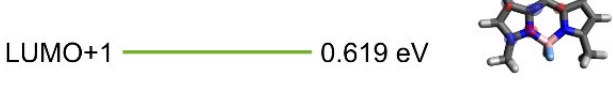

LUMO $-1.963 \mathrm{eV}$

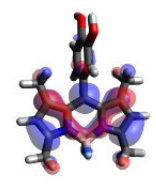

HOMO $--6.768 \mathrm{eV}$

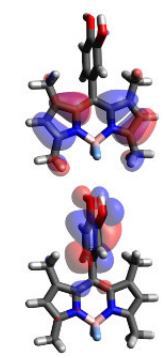

Figure S18. a) Molecular structure, b) optimized structure, c) frontier molecular orbitals and corresponding energy levels, and calculated $\Delta \mathrm{E}$ of B16 in the ground state in ethanol at M062X/Def2SVP level. 
a

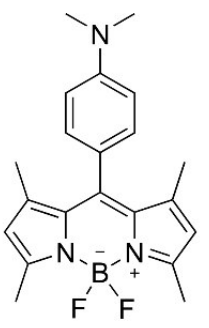

b

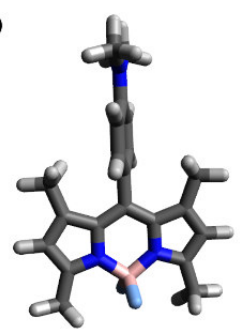

C

LUMO+1 $0.599 \mathrm{eV}$

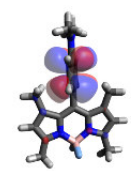

LUMO $-1.921 \mathrm{eV}$
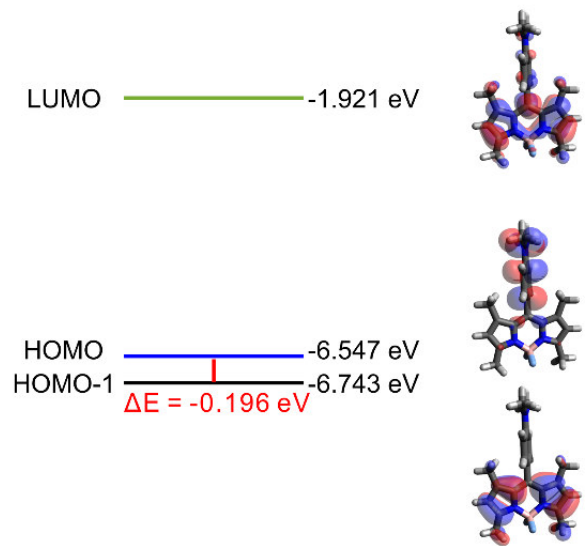

Figure S19. a) Molecular structure, b) optimized structure, c) frontier molecular orbitals and corresponding energy levels, and calculated $\Delta \mathrm{E}$ of B17 in the ground state in methanol at M062X/Def2SVP level.

a

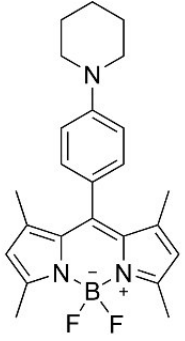

b

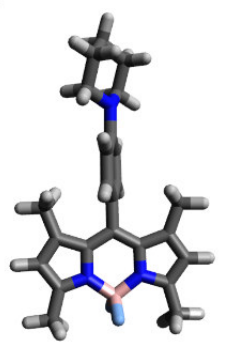

C

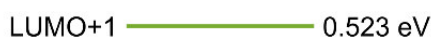

LUMO
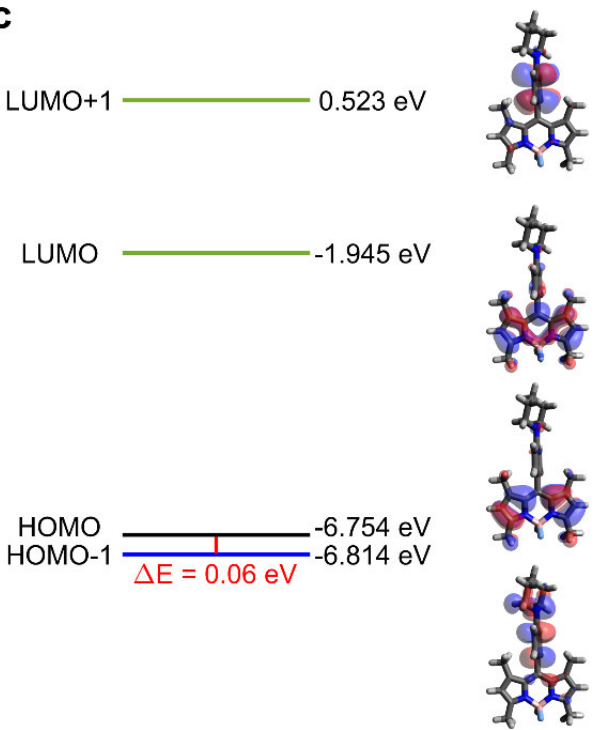

Figure S20. a) Molecular structure, b) optimized structure, c) frontier molecular orbitals and corresponding energy levels, and calculated $\Delta \mathrm{E}$ of B18 in the ground state in methanol at M062X/Def2SVP level. 


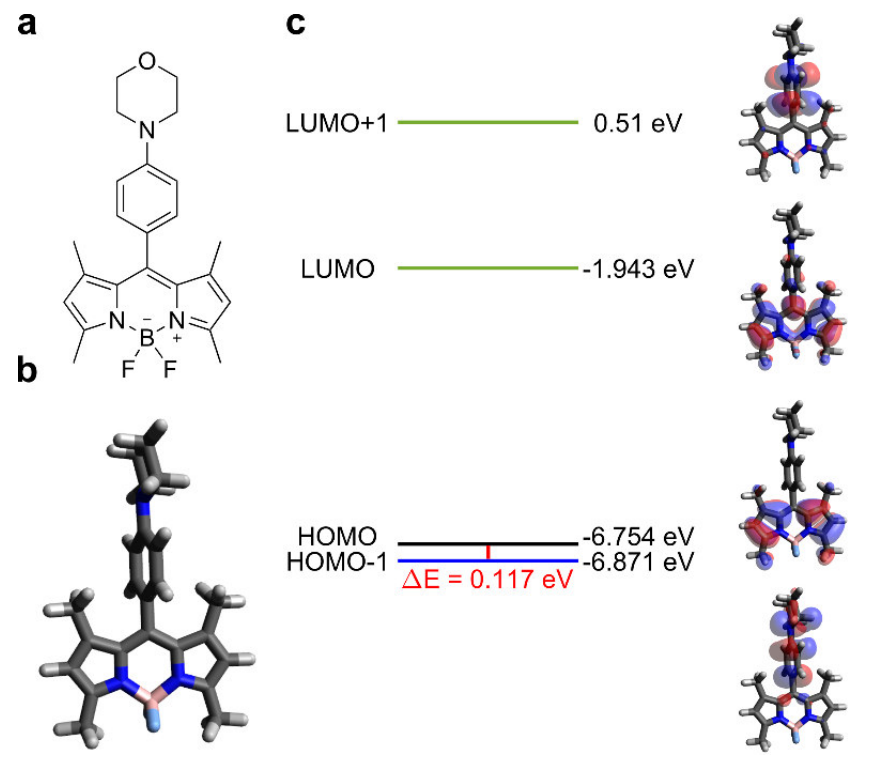

Figure S21. a) Molecular structure, b) optimized structure, c) frontier molecular orbitals and corresponding energy levels, and calculated $\Delta \mathrm{E}$ of B19 in the ground state in methanol at M062X/Def2SVP level.
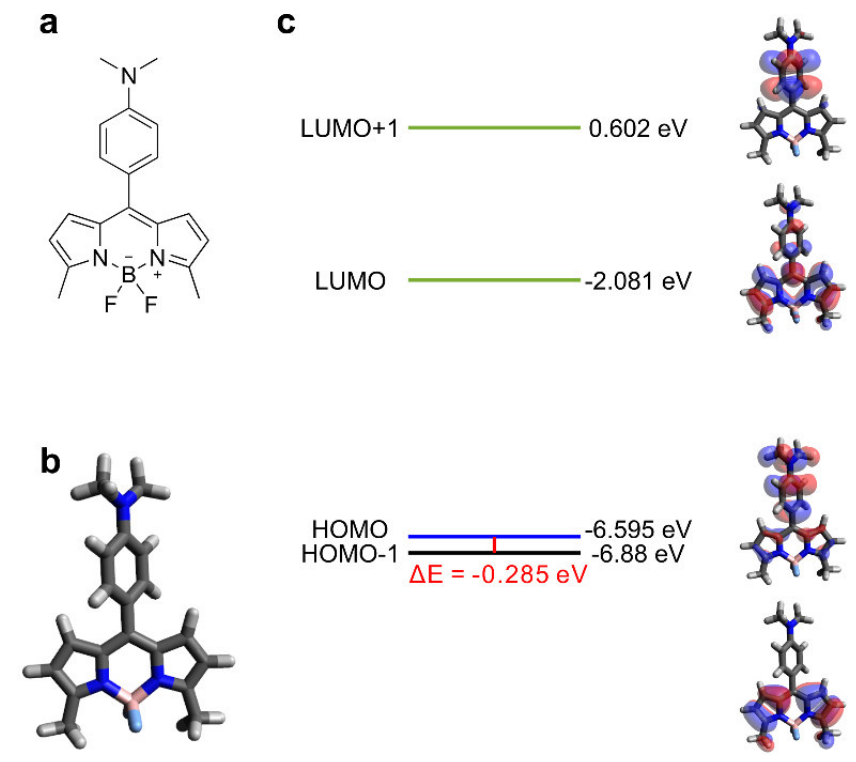

Figure S22. a) Molecular structure, b) optimized structure, c) frontier molecular orbitals and corresponding energy levels, and calculated $\Delta \mathrm{E}$ of B20 in the ground state in methanol at M062X/Def2SVP level. 
a
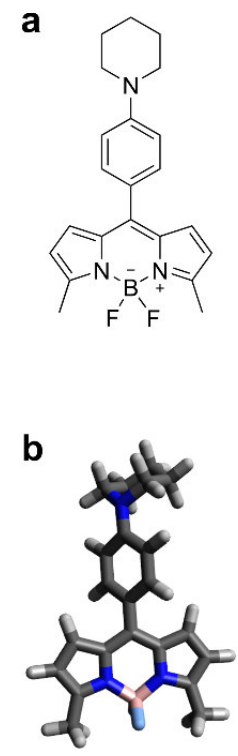

C

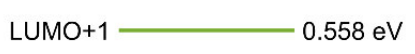

LUMO
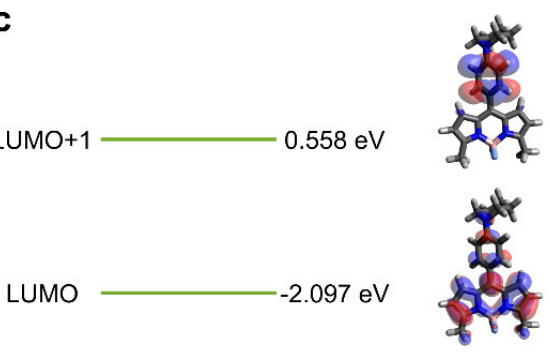

HOMO
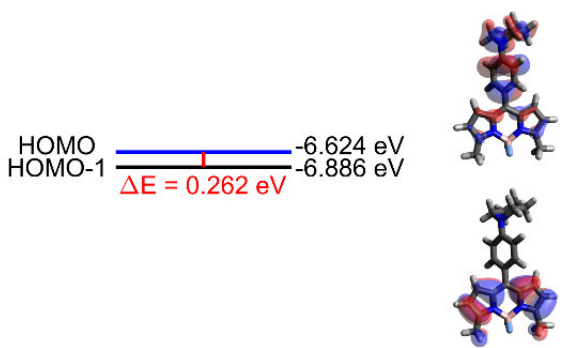

Figure S23. a) Molecular structure, b) optimized structure, c) frontier molecular orbitals and corresponding energy levels, and calculated $\Delta \mathrm{E}$ of B21 in the ground state in methanol at M062X/Def2SVP level.

a
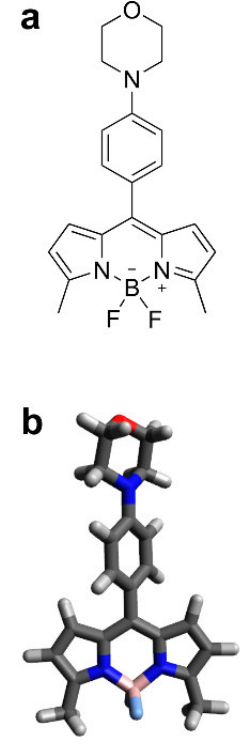

C

LUMO+1 $0.524 \mathrm{eV}$
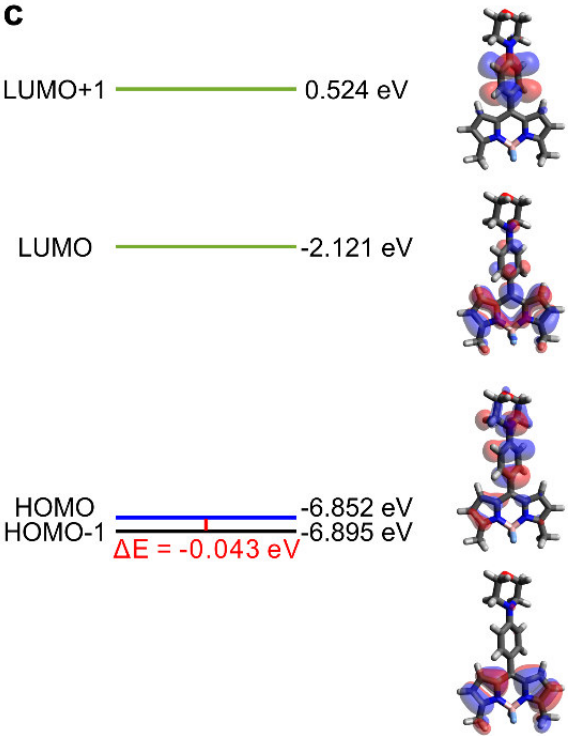

Figure S24. a) Molecular structure, b) optimized structure, c) frontier molecular orbitals and corresponding energy levels, and calculated $\Delta \mathrm{E}$ of B22 in the ground state in methanol at M062X/Def2SVP level. 
a<smiles></smiles>

b

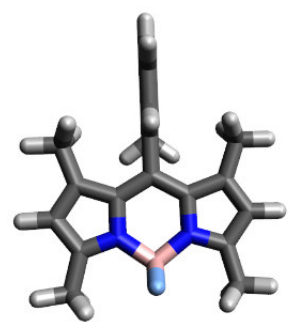

C

LUMO+1 $0.422 \mathrm{eV}$

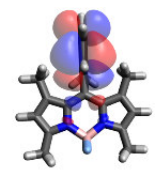

LUMO $-1.951 \mathrm{eV}$
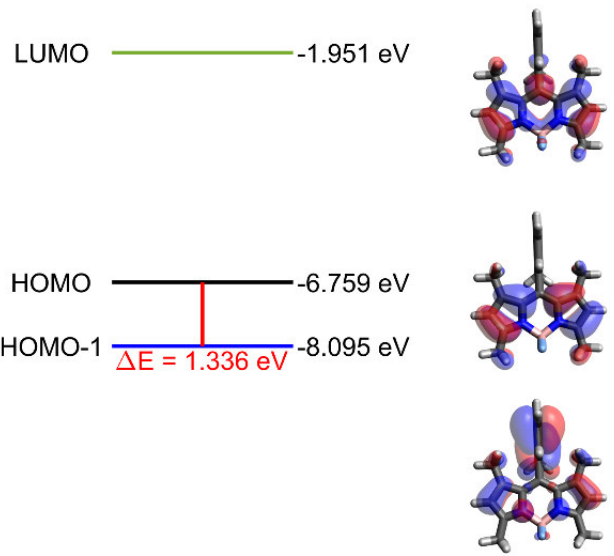

Figure S25. a) Molecular structure, b) optimized structure, c) frontier molecular orbitals and corresponding energy levels, and calculated $\Delta \mathrm{E}$ of B23 in the ground state in methanol at M062X/Def2SVP level.

a

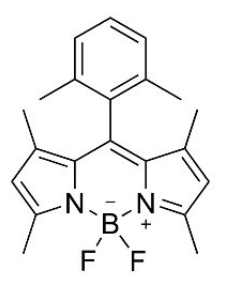

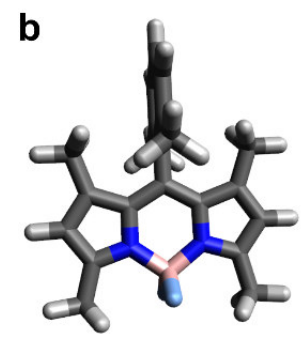

C
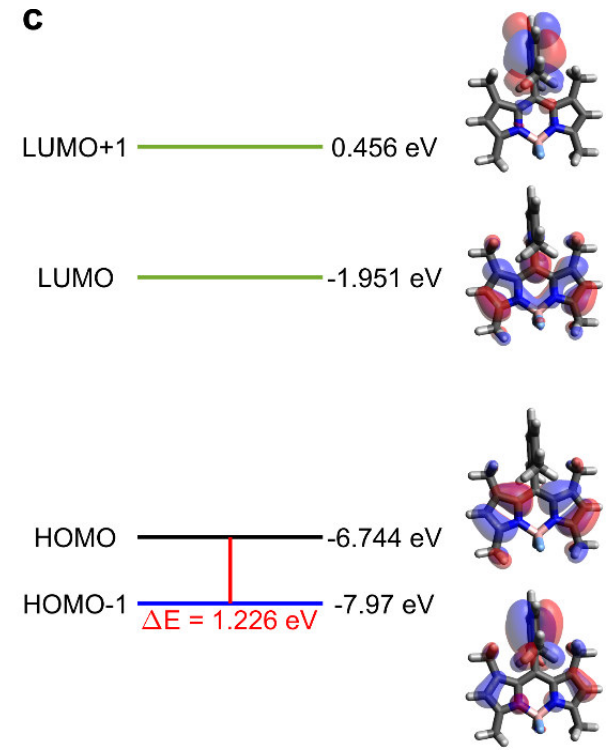

Figure S26. a) Molecular structure, b) optimized structure, c) frontier molecular orbitals and corresponding energy levels, and calculated $\Delta \mathrm{E}$ of B24 in the ground state in methanol at M062X/Def2SVP level. 
a<smiles></smiles>

C

LUMO+1

LUMO

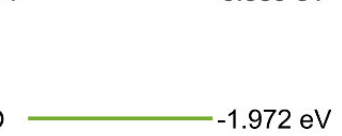

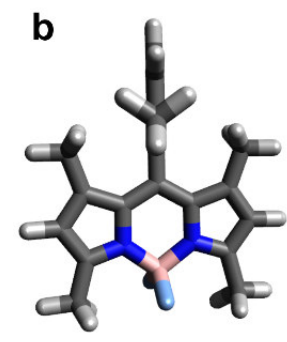

HOMO $\longrightarrow-6.75 \mathrm{eV}$

HOMO-1 $\frac{}{\Delta \mathrm{E}=0.926 \mathrm{eV}}-7.676 \mathrm{eV}$
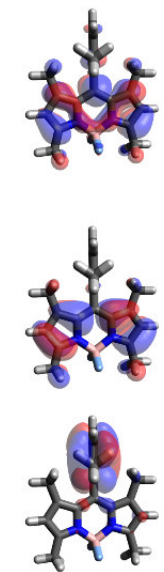

Figure S27. a) Molecular structure, b) optimized structure, c) frontier molecular orbitals and corresponding energy levels, and calculated $\Delta \mathrm{E}$ of B25 in the ground state in methanol at M062X/Def2SVP level.

a<smiles></smiles>

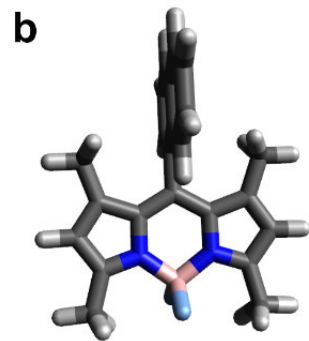

C<smiles>CC(C)O[GaH2]</smiles>

LUMO
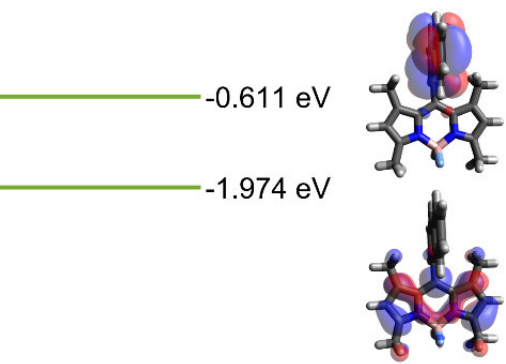

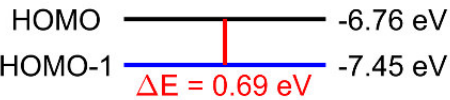
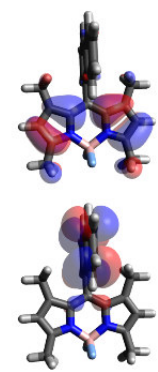

Figure S28. a) Molecular structure, b) optimized structure, c) frontier molecular orbitals and corresponding energy levels, and calculated $\Delta \mathrm{E}$ of B26 in the ground state in methanol at M062X/Def2SVP level. 
a<smiles>COc1c(C)cccc1C1=C2C(C)=CC(C)=[N+]2[B-](F)(F)n2c(C)cc(C)c21</smiles>

b

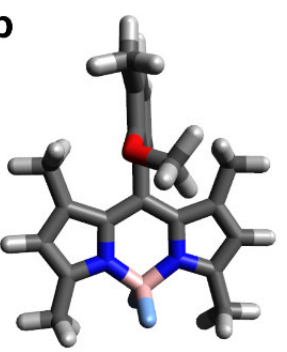

C

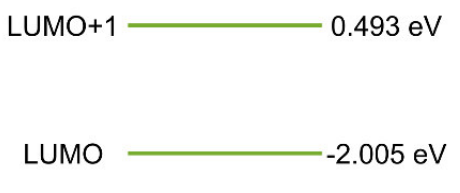

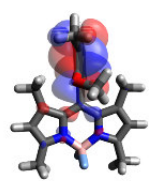

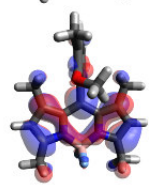

HOMO $-6.754 \mathrm{eV}$
HOMO-1 $\frac{1.277 \mathrm{eV}}{\Delta \mathrm{E}=1.031 \mathrm{eV}}$
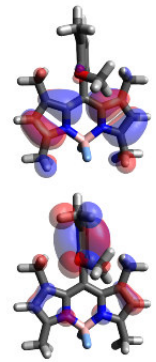

Figure S29. a) Molecular structure, b) optimized structure, c) frontier molecular orbitals and corresponding energy levels, and calculated $\Delta \mathrm{E}$ of B27 in the ground state in methanol at M062X/Def2SVP level.

a<smiles>COc1cccc(C2=C3C(C)=CC(C)=[N+]3[B-](F)(F)n3c(C)cc(C)c32)c1OC</smiles>

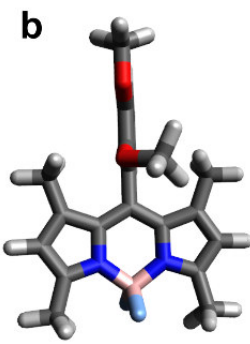

C

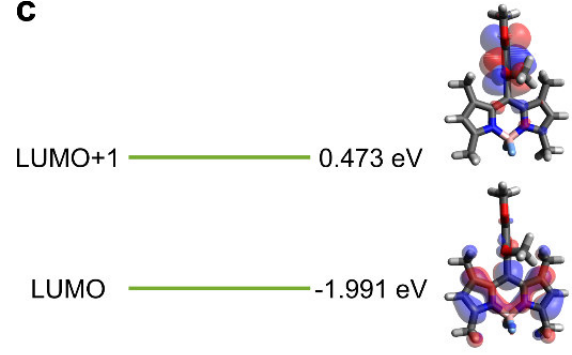

HOMO
HOMO-1
$\frac{\mathrm{E}=0.757 \mathrm{eV}}{\Delta \mathrm{E}=0.868 \mathrm{eV}}-7.625 \mathrm{eV}$

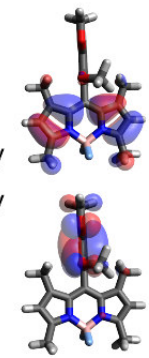

Figure S30. a) Molecular structure, b) optimized structure, c) frontier molecular orbitals and corresponding energy levels, and calculated $\Delta \mathrm{E}$ of B28 in the ground state in methanol at M062X/Def2SVP level. 

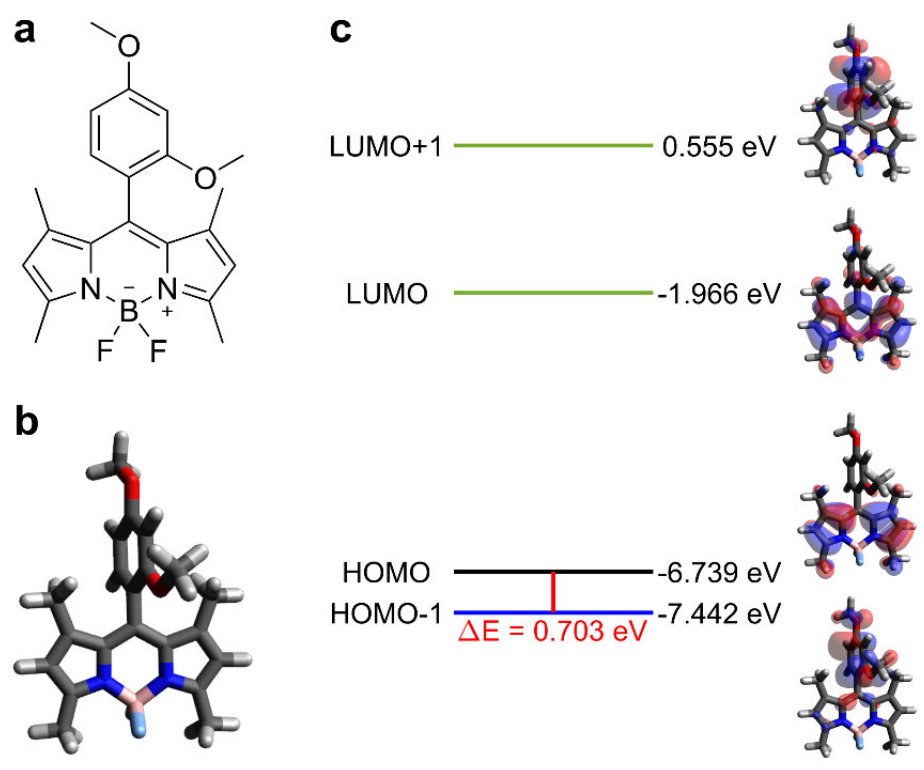

Figure S31. a) Molecular structure, b) optimized structure, c) frontier molecular orbitals and corresponding energy levels, and calculated $\Delta \mathrm{E}$ of B29 in the ground state in methanol at M062X/Def2SVP level.

a<smiles></smiles>

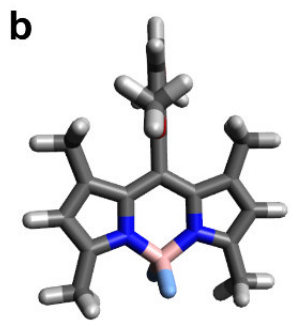

C

LUMO+1 $0.299 \mathrm{eV}$

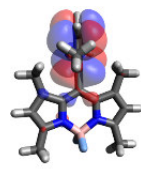

LUMO

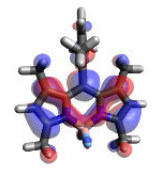

HOMO
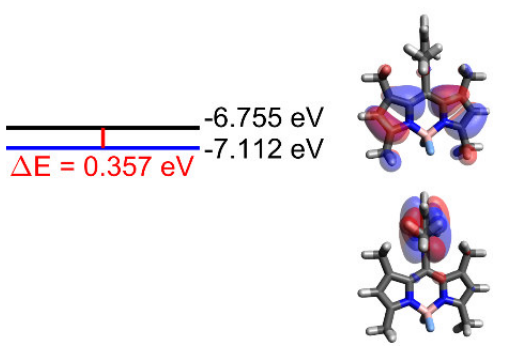

Figure S32. a) Molecular structure, b) optimized structure, c) frontier molecular orbitals and corresponding energy levels, and calculated $\Delta \mathrm{E}$ of $\mathrm{B} 30$ in the ground state in methanol at M062X/Def2SVP level. 
a<smiles>CC1=CC(C)=[N+]2B(F)n3c(C)cc(C)c3C(c3c4ccccc4cc4ccccc34)=C12</smiles>

C

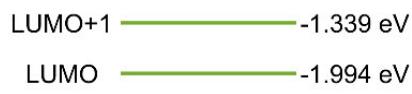

b

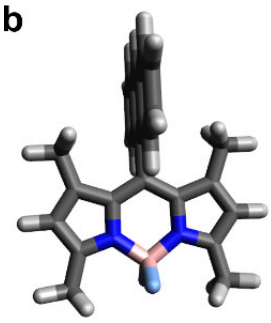

HOMO HOMO-
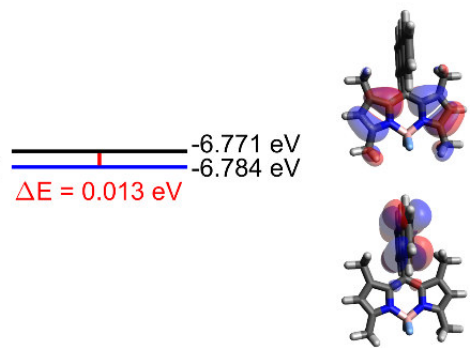

Figure S33. a) Molecular structure, b) optimized structure, c) frontier molecular orbitals and corresponding energy levels, and calculated $\Delta \mathrm{E}$ of B31 in the ground state in methanol at M062X/Def2SVP level.

a<smiles></smiles>

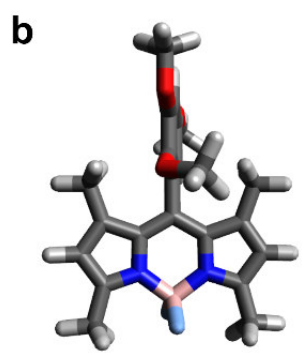

C
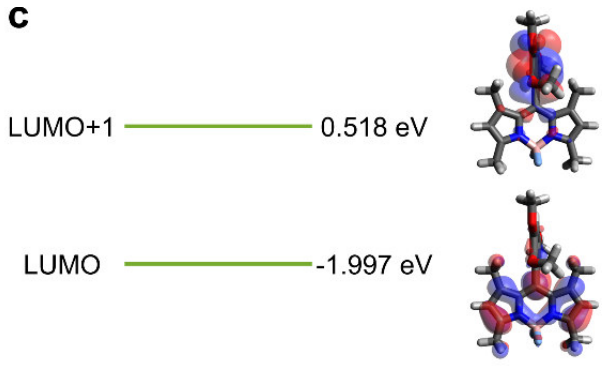

HOMO $\frac{}{\mathrm{HOMO}}-6.758 \mathrm{eV}$
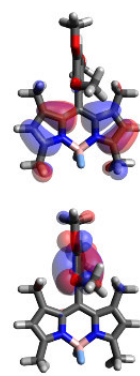

Figure S34. a) Molecular structure, b) optimized structure, c) frontier molecular orbitals and corresponding energy levels, and calculated $\Delta \mathrm{E}$ of $\mathrm{B} 32$ in the ground state in methanol at M062X/Def2SVP level. 
a

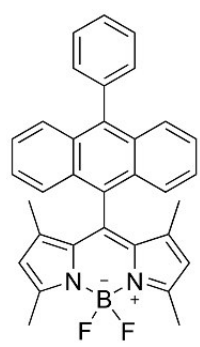

b

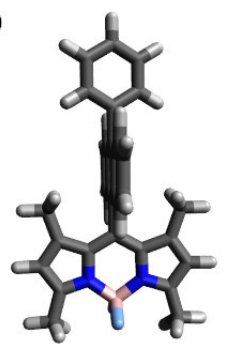

C
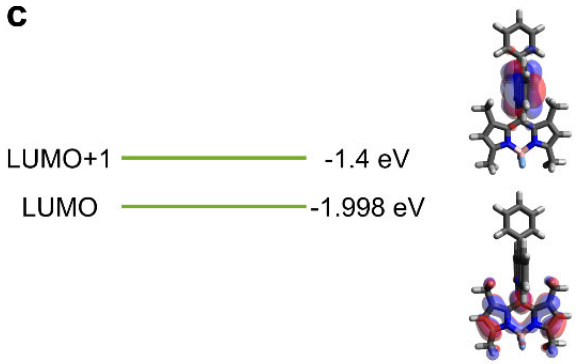

HOMO
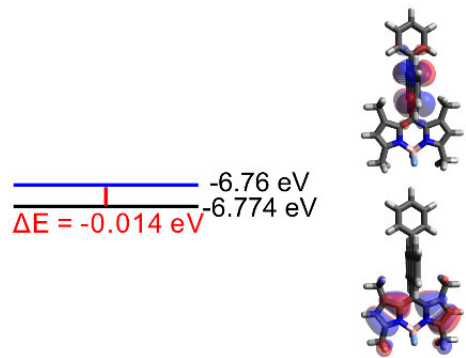

Figure S35. a) Molecular structure, b) optimized structure, c) frontier molecular orbitals and corresponding energy levels, and calculated $\Delta \mathrm{E}$ of B33 in the ground state in methanol at M062X/Def2SVP level.
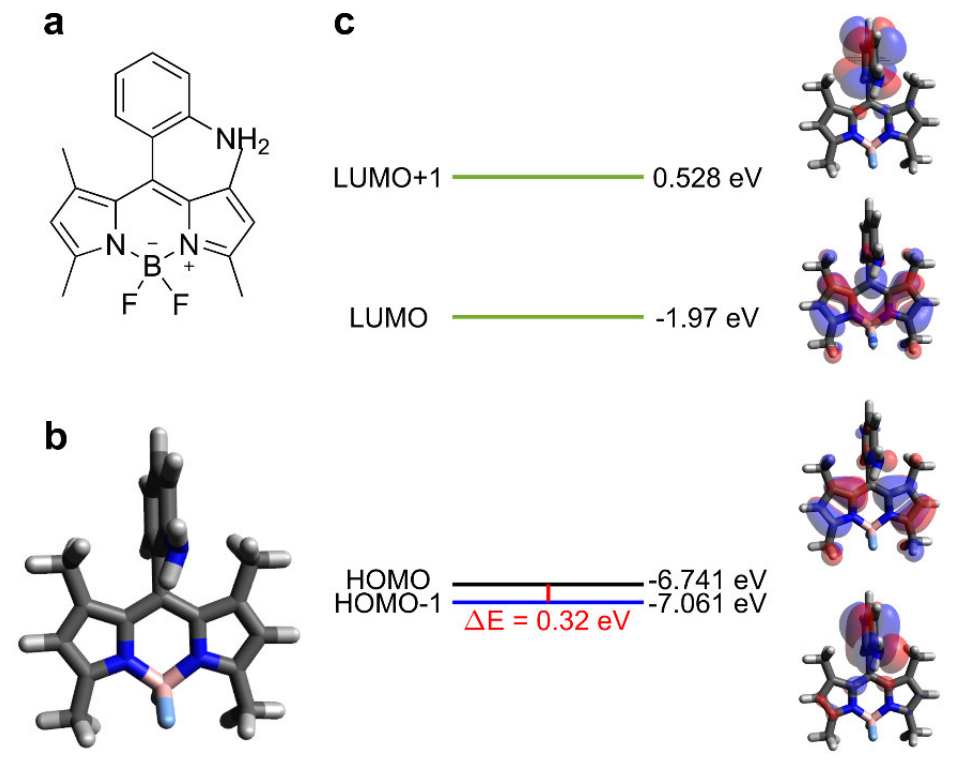

Figure S36. a) Molecular structure, b) optimized structure, c) frontier molecular orbitals and corresponding energy levels, and calculated $\Delta \mathrm{E}$ of B34 in the ground state in methanol at M062X/Def2SVP level. 
a<smiles></smiles>

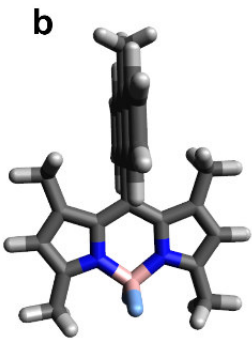

C
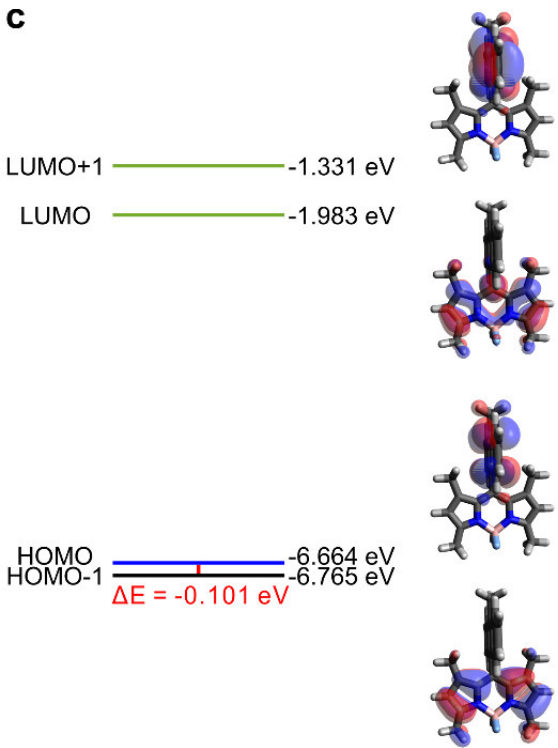

Figure S37. a) Molecular structure, b) optimized structure, c) frontier molecular orbitals and corresponding energy levels, and calculated $\Delta \mathrm{E}$ of B35 in the ground state in methanol at M062X/Def2SVP level.

a<smiles>COc1ccc(N)cc1C1=C2C(C)=CC(C)=[N+]2[B-](F)(F)n2c(C)cc(C)c21</smiles>

b

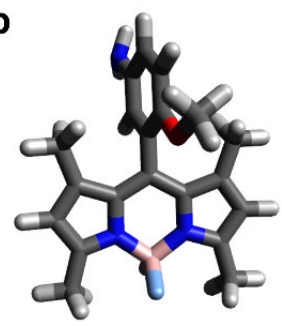

C
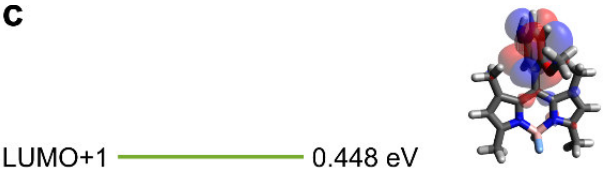

LUMO $-1.961 \mathrm{eV}$
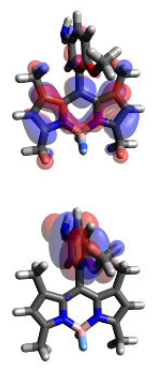

$\stackrel{\mathrm{HOMO}}{\mathrm{HOMO}} \frac{-6.616 \mathrm{eV}}{\overline{\Delta E=-0.125 \mathrm{eV}}-6.741 \mathrm{eV}}$

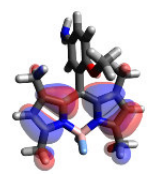

Figure S38. a) Molecular structure, b) optimized structure, c) frontier molecular orbitals and corresponding energy levels, and calculated $\Delta \mathrm{E}$ of B36 in the ground state in methanol at M062X/Def2SVP level. 


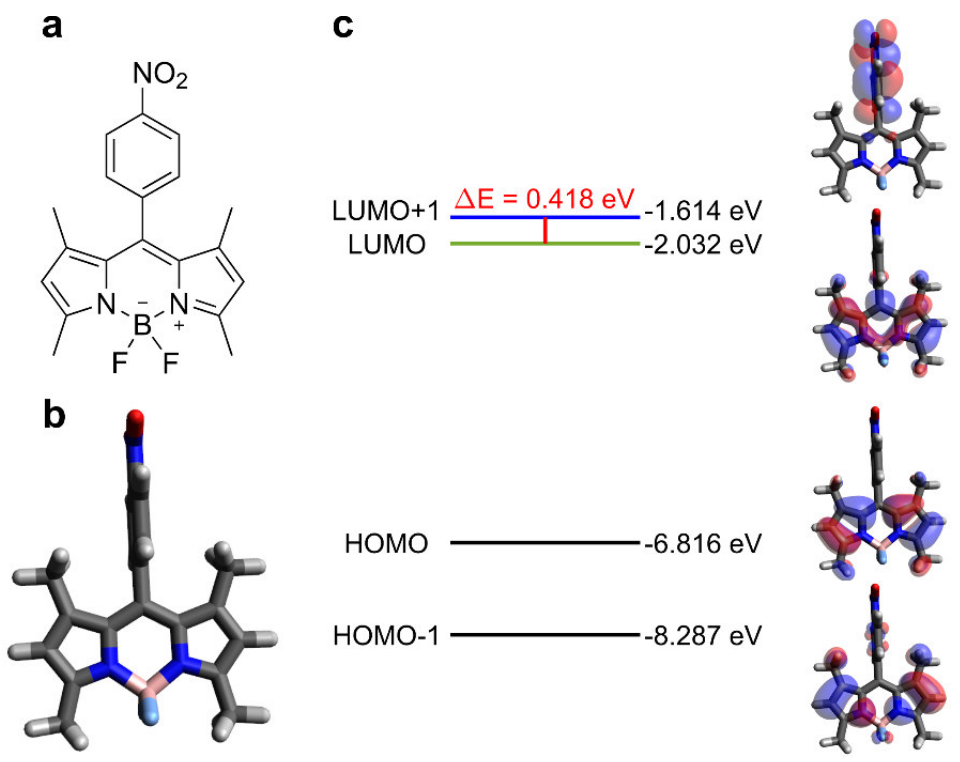

Figure S39. a) Molecular structure, b) optimized structure, c) frontier molecular orbitals and corresponding energy levels, and calculated $\Delta \mathrm{E}$ of B37 in the ground state in methanol at M062X/Def2SVP level.
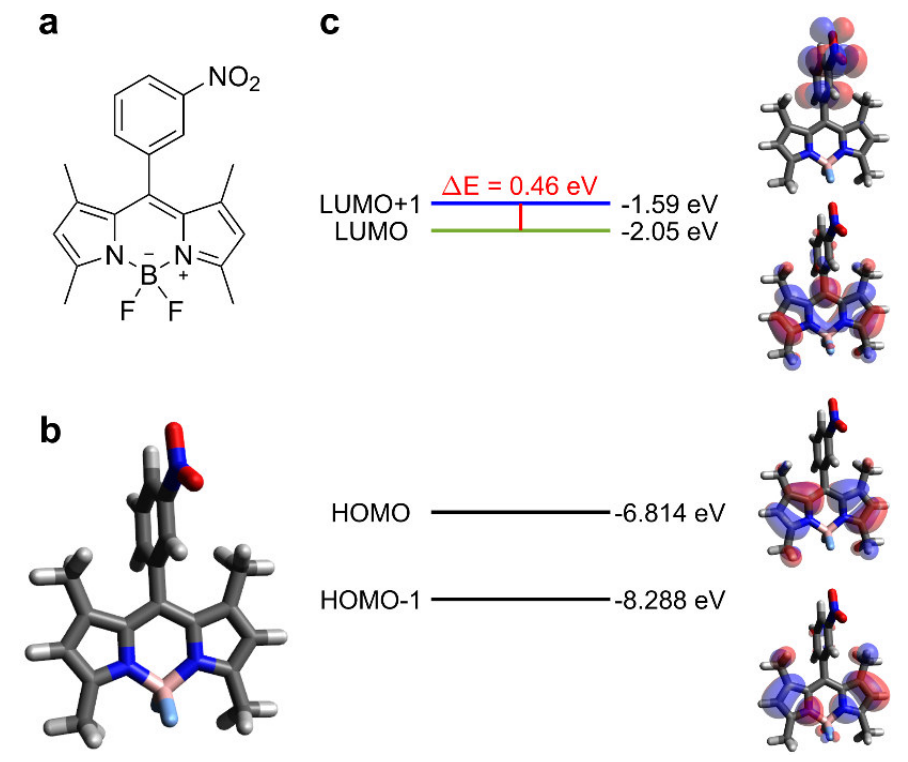

Figure S40. a) Molecular structure, b) optimized structure, c) frontier molecular orbitals and corresponding energy levels, and calculated $\Delta \mathrm{E}$ of B38 in the ground state in methanol at M062X/Def2SVP level. 
a<smiles></smiles>
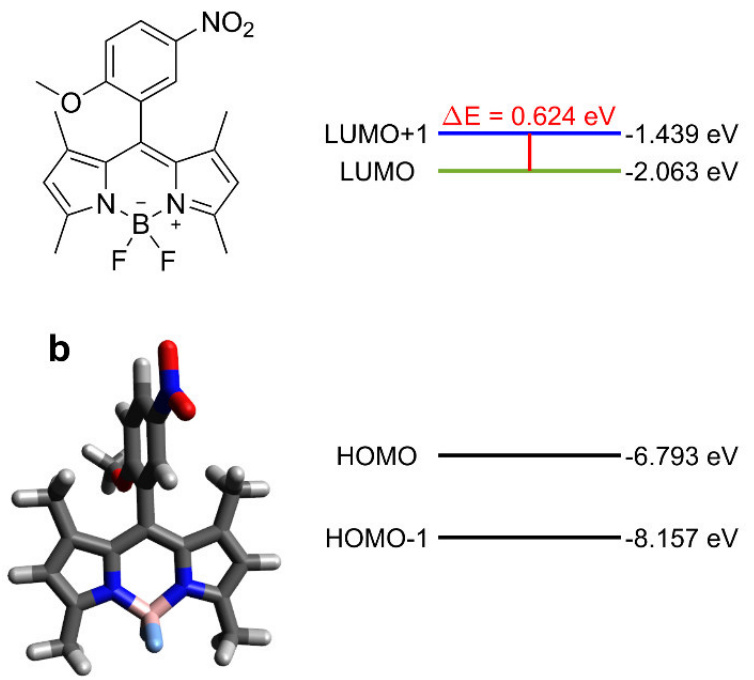

C
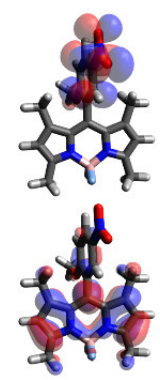

HOMO $-6.793 \mathrm{eV}$

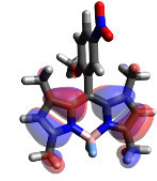

HOMO-1 - $-8.157 \mathrm{eV}$

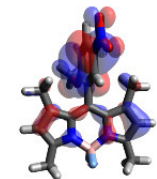

Figure S41. a) Molecular structure, b) optimized structure, c) frontier molecular orbitals and corresponding energy levels, and calculated $\Delta \mathrm{E}$ of B39 in the ground state in methanol at M062X/Def2SVP level.<smiles></smiles>
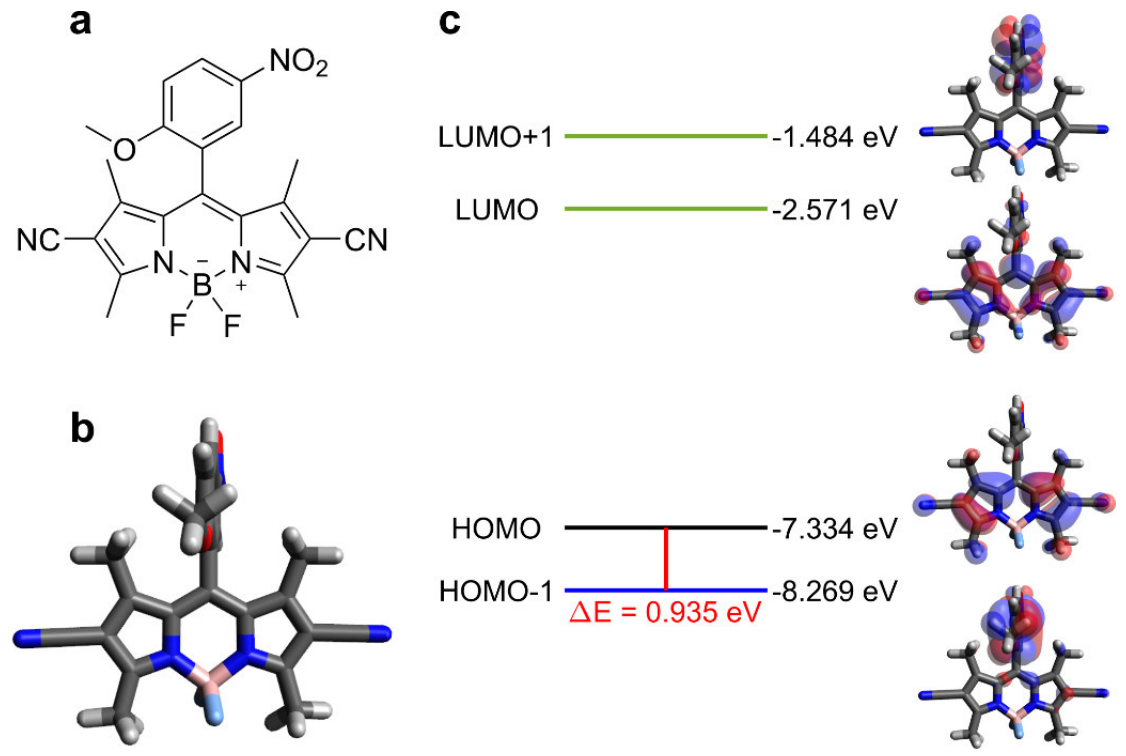

Figure S42. a) Molecular structure, b) optimized structure, c) frontier molecular orbitals and corresponding energy levels, and calculated $\Delta \mathrm{E}$ of $\mathrm{B} 40$ in the ground state in methanol at M062X/Def2SVP level. 

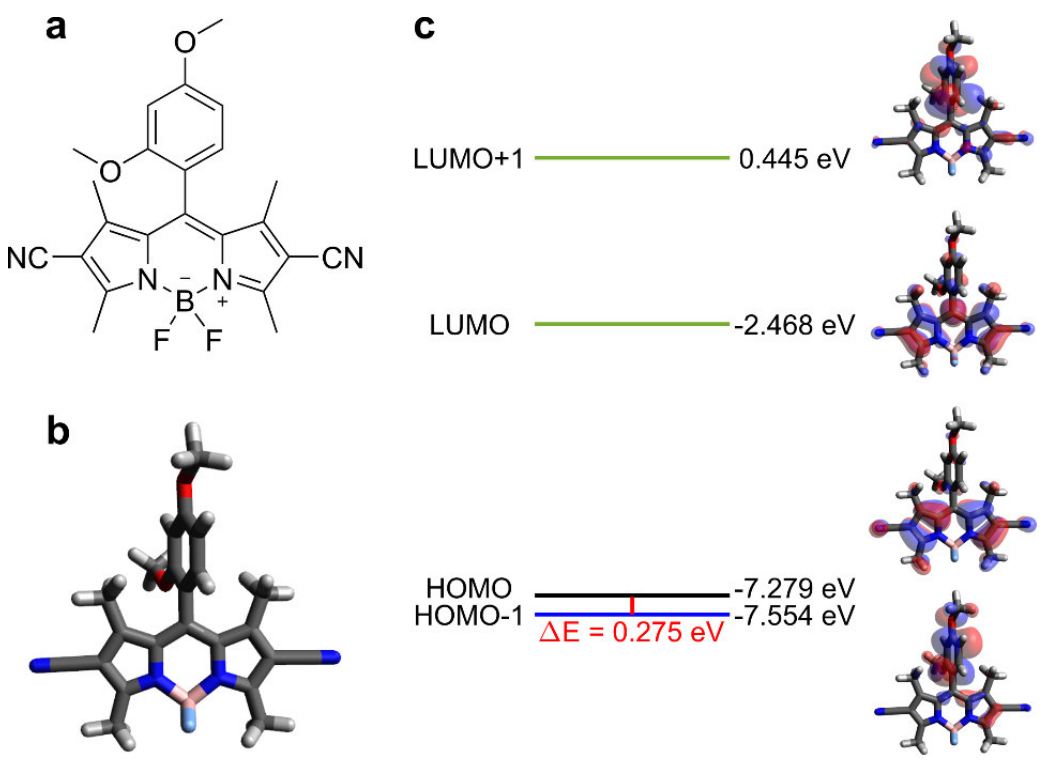

Figure S43. a) Molecular structure, b) optimized structure, c) frontier molecular orbitals and corresponding energy levels, and calculated $\Delta \mathrm{E}$ of B41 in the ground state in methanol at M062X/Def2SVP level.
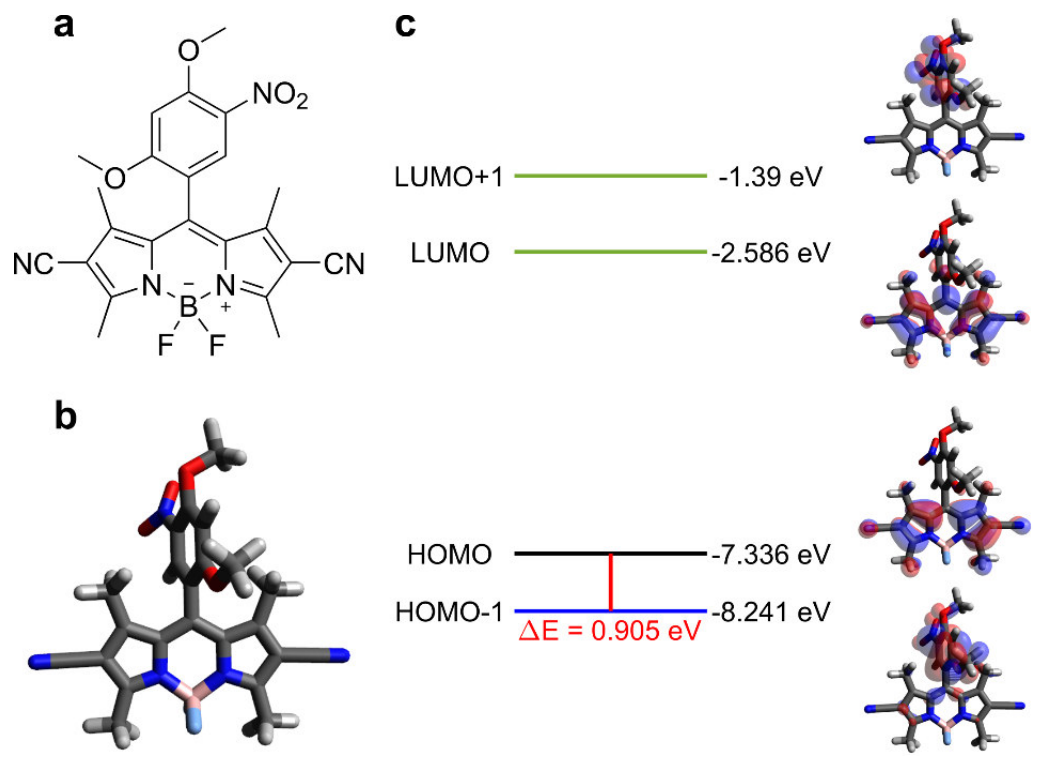

Figure S44. a) Molecular structure, b) optimized structure, c) frontier molecular orbitals and corresponding energy levels, and calculated $\Delta \mathrm{E}$ of B42 in the ground state in methanol at M062X/Def2SVP level. 
a<smiles></smiles>

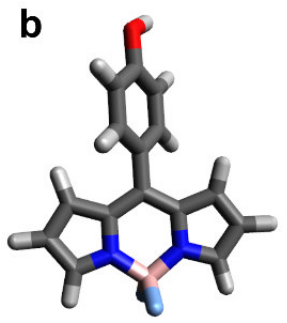

C
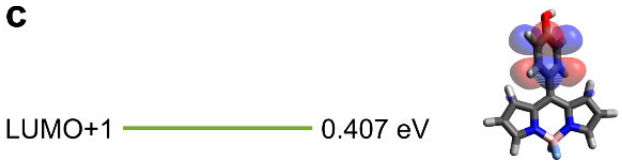

LUMO

$-2.295 \mathrm{eV}$

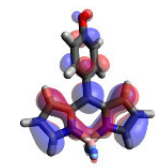

$\mathrm{HOMO} \frac{\mathrm{H}}{\mathrm{HOMO}}-7.241 \mathrm{eV}$

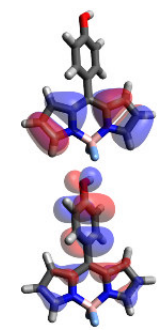

Figure S45. a) Molecular structure, b) optimized structure, c) frontier molecular orbitals and corresponding energy levels, and calculated $\Delta \mathrm{E}$ of $\mathrm{B} 43$ in the ground state in methanol at M062X/Def2SVP level.

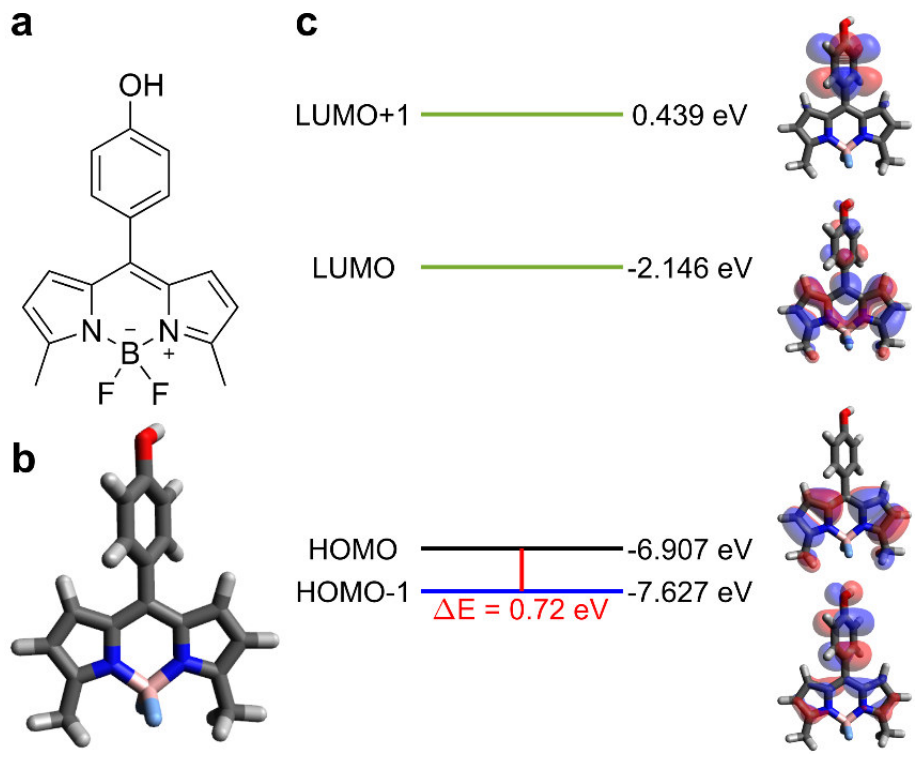

Figure S46. a) Molecular structure, b) optimized structure, c) frontier molecular orbitals and corresponding energy levels, and calculated $\Delta \mathrm{E}$ of B44 in the ground state in methanol at M062X/Def2SVP level. 
a<smiles>Oc1ccc(C2=C3C=CC(Cl)=[N+]3[B-](F)(F)n3c(Cl)ccc32)cc1</smiles>

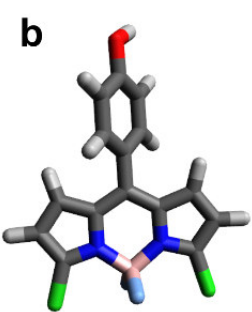

C

LUMO $+1 \longrightarrow 0.377 \mathrm{eV}$

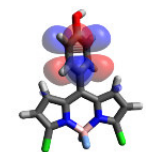

LUMO

$-2.448 \mathrm{eV}$

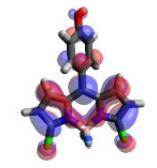

HOMO

$-7.216 \mathrm{eV}$
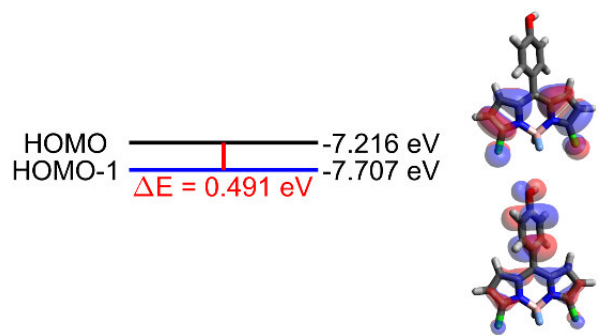

Figure S47. a) Molecular structure, b) optimized structure, c) frontier molecular orbitals and corresponding energy levels, and calculated $\Delta \mathrm{E}$ of B45 in the ground state in methanol at M062X/Def2SVP level.

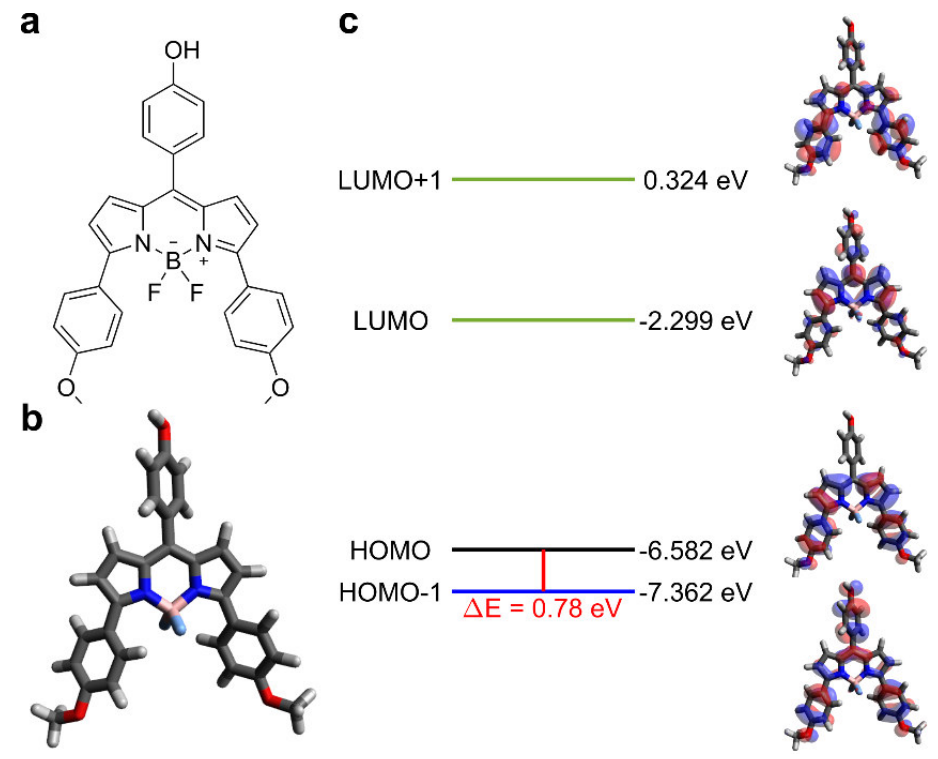

Figure S48. a) Molecular structure, b) optimized structure, c) frontier molecular orbitals and corresponding energy levels, and calculated $\Delta \mathrm{E}$ of B46 in the ground state in methanol at M062X/Def2SVP level. 
a<smiles></smiles>

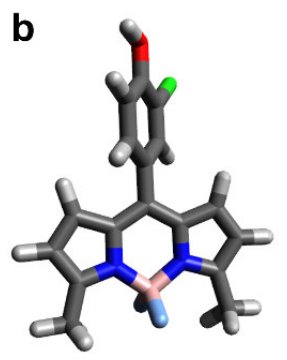

C

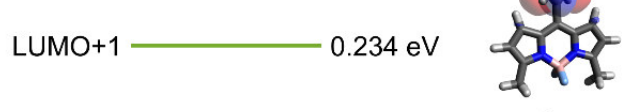

LUMO - $-2.192 \mathrm{eV}$
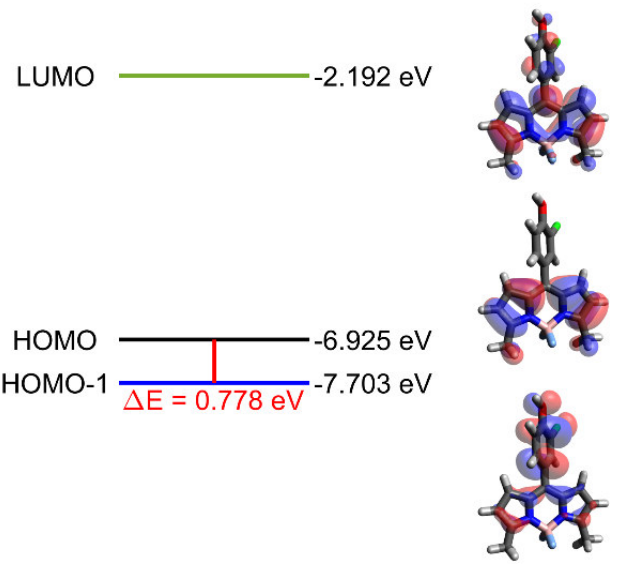

Figure S49. a) Molecular structure, b) optimized structure, c) frontier molecular orbitals and corresponding energy levels, and calculated $\Delta \mathrm{E}$ of B47 in the ground state in methanol at M062X/Def2SVP level.<smiles></smiles>

\section{C}
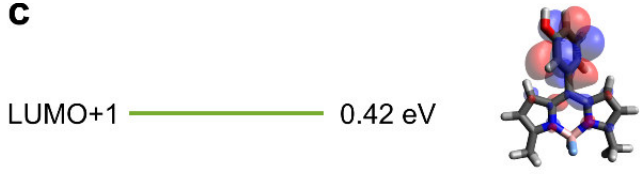

LUMO $-2.184 \mathrm{eV}$
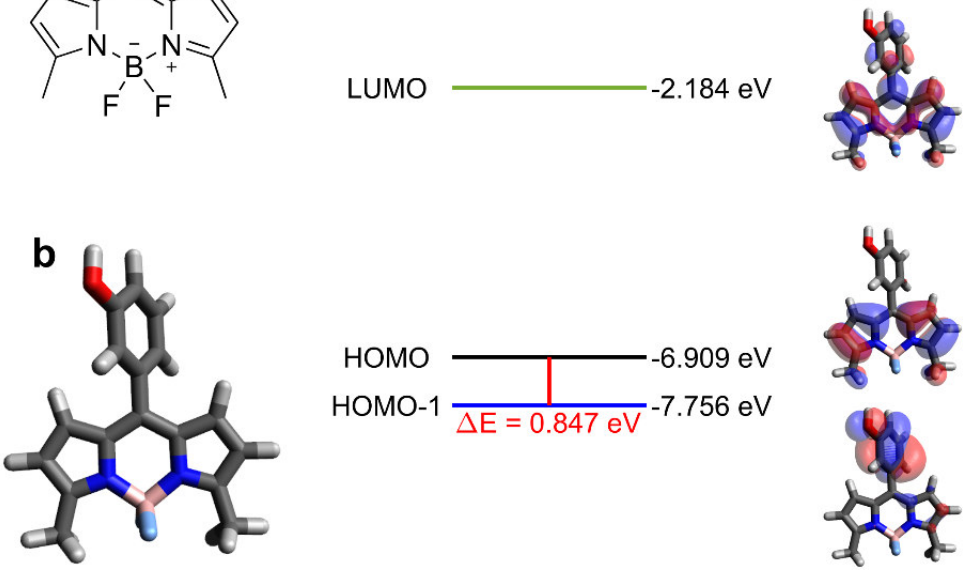

Figure S50. a) Molecular structure, b) optimized structure, c) frontier molecular orbitals and corresponding energy levels, and calculated $\Delta \mathrm{E}$ of B48 in the ground state in methanol at M062X/Def2SVP level. 

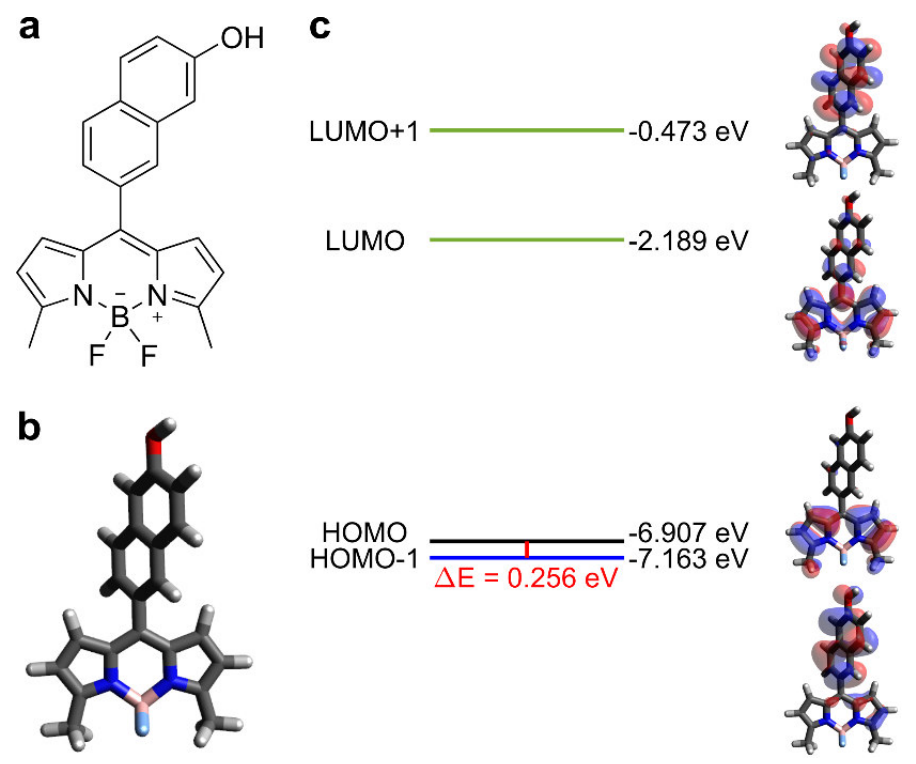

Figure S51. a) Molecular structure, b) optimized structure, c) frontier molecular orbitals and corresponding energy levels, and calculated $\Delta \mathrm{E}$ of B49 in the ground state in methanol at M062X/Def2SVP level.
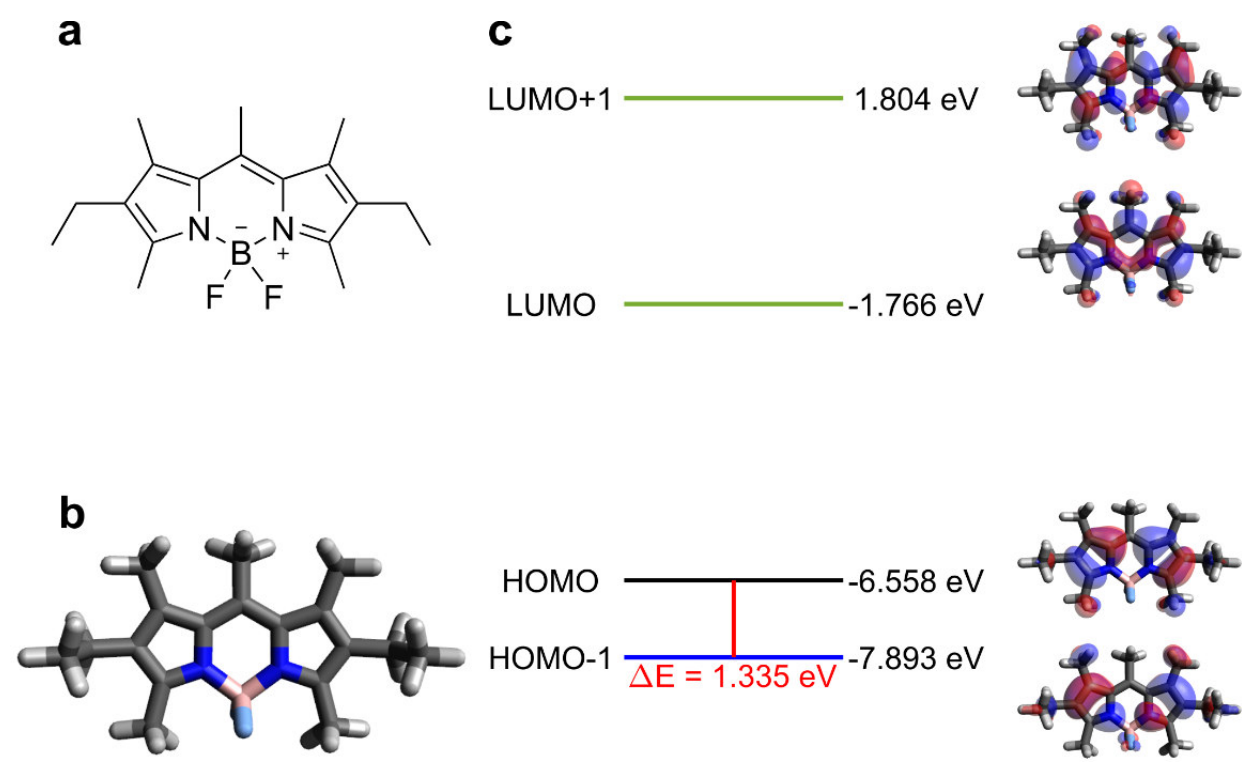

Figure S52. a) Molecular structure, b) optimized structure, c) frontier molecular orbitals and corresponding energy levels, and calculated $\Delta \mathrm{E}$ of B50 in the ground state in acetonitrile at M062X/Def2SVP level. 
a<smiles></smiles>

b

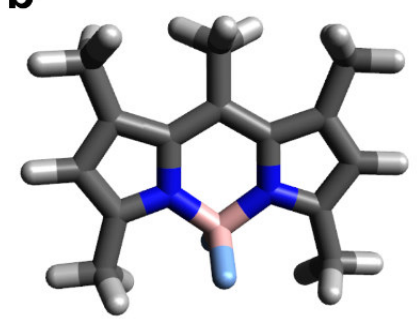

C

LUMO+1

$1.773 \mathrm{eV}$

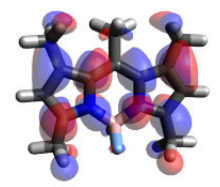

$1.837 \mathrm{eV}$

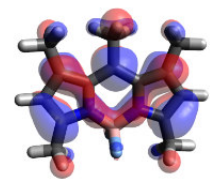

LUMO

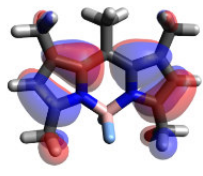

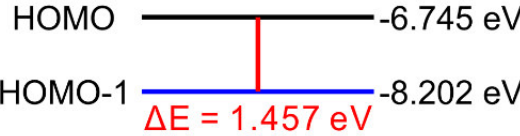

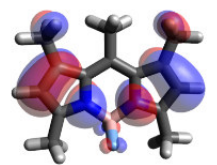

Figure S53. a) Molecular structure, b) optimized structure, c) frontier molecular orbitals and corresponding energy levels, and calculated $\Delta \mathrm{E}$ of B51 in the ground state in acetonitrile at M062X/Def2SVP level.

a<smiles>CC1=C(Cl)C(C)=[N+]2C1=C(C)c1c(C)cc(C)n1[B-]2(F)F</smiles>

b

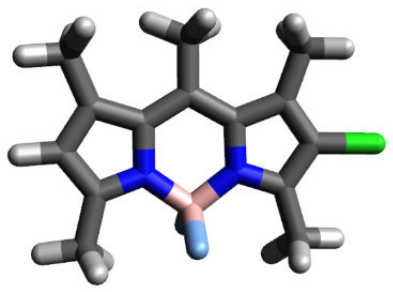

C

LUMO+1 $1.655 \mathrm{eV}$

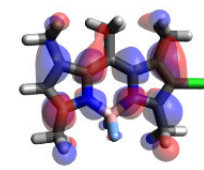

LUMO $-1.943 \mathrm{eV}$

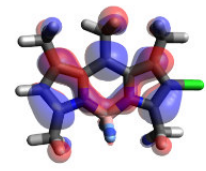

\begin{tabular}{l|l} 
HOMO & $-6.828 \mathrm{eV}$ \\
HOMO-1 & $\frac{1}{\Delta E=1.248 \mathrm{eV}}-8.076 \mathrm{eV}$
\end{tabular}
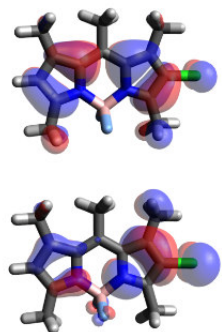

Figure S54. a) Molecular structure, b) optimized structure, c) frontier molecular orbitals and corresponding energy levels, and calculated $\Delta \mathrm{E}$ of B52 in the ground state in acetonitrile at M062X/Def2SVP level. 
<smiles></smiles>

C
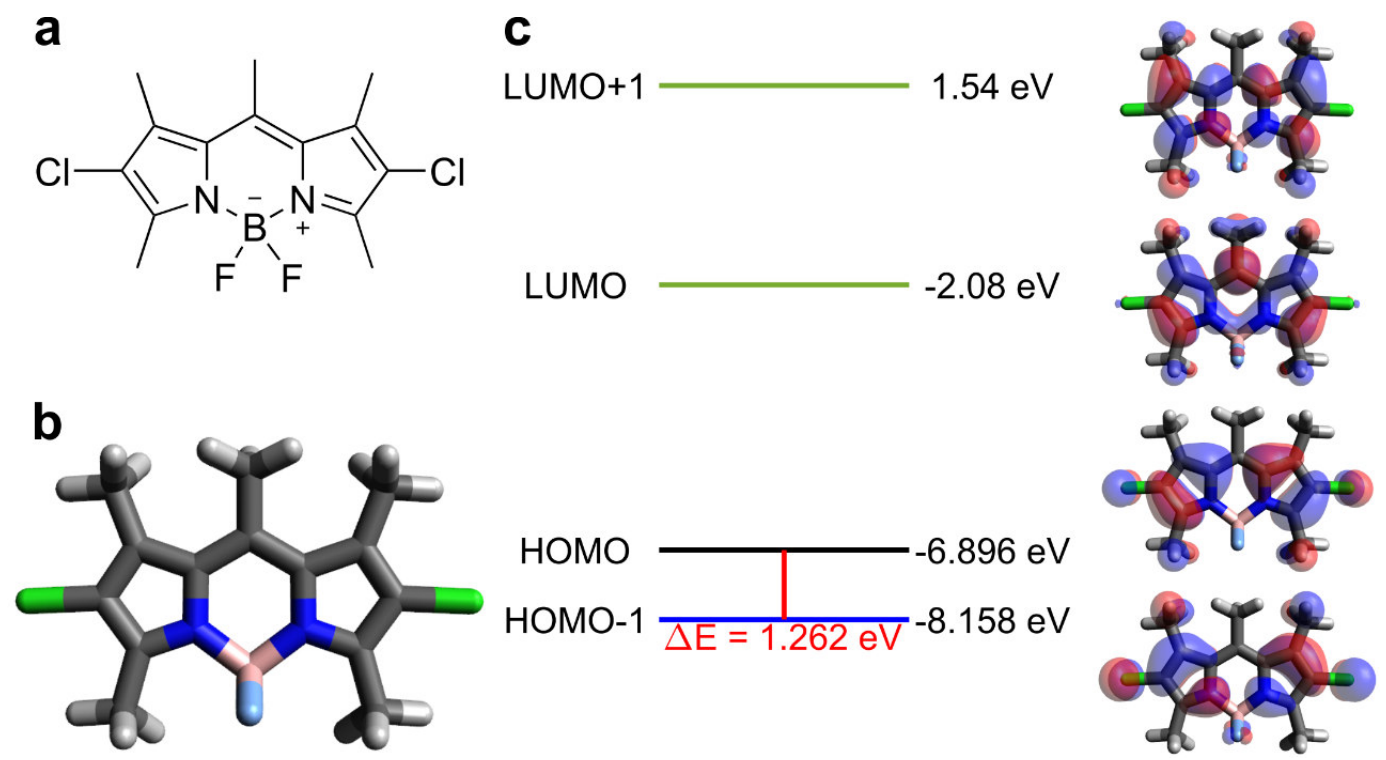

Figure S55. a) Molecular structure, b) optimized structure, c) frontier molecular orbitals and corresponding energy levels, and calculated $\triangle \mathrm{E}$ of B53 in the ground state in acetonitrile at M062X/Def2SVP level.

a<smiles></smiles>

LUMO $-1.962 \mathrm{eV}$

$1.827 \mathrm{eV}$ LUMO+1

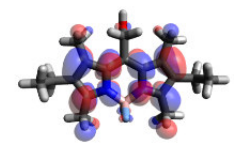

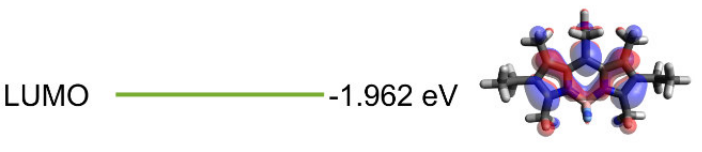
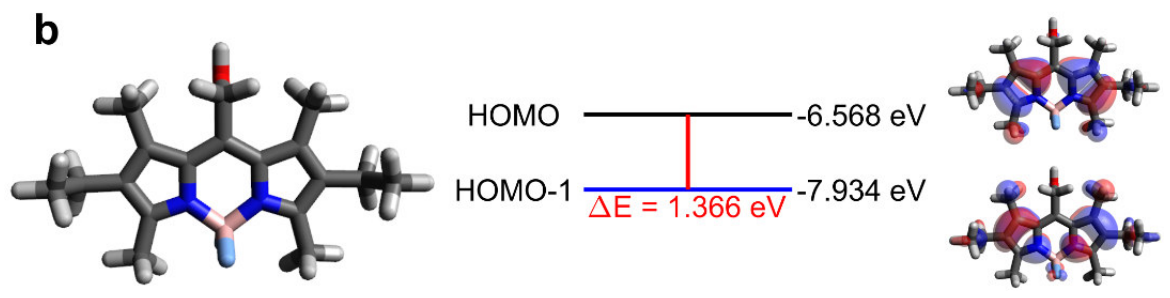

Figure S56. a) Molecular structure, b) optimized structure, c) frontier molecular orbitals and corresponding energy levels, and calculated $\Delta \mathrm{E}$ of B54 in the ground state in acetonitrile at M062X/Def2SVP level. 
a<smiles></smiles>

C

LUMO+1 $1.787 \mathrm{eV}$
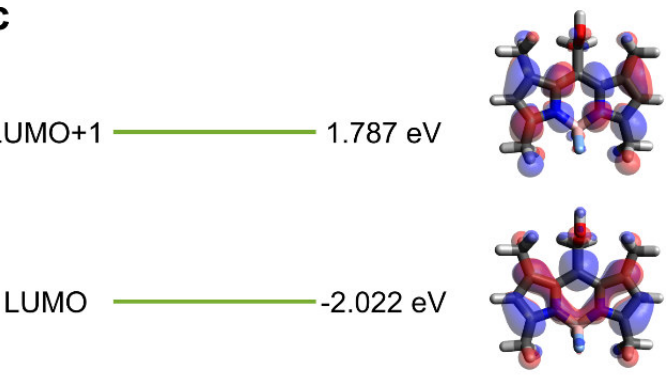
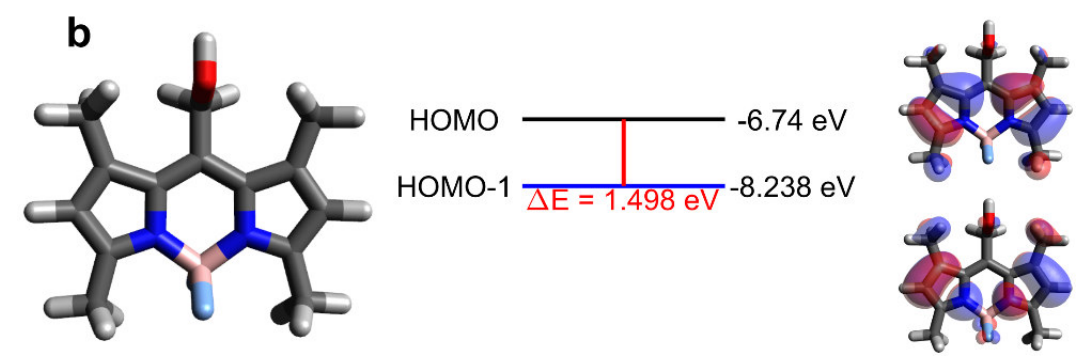

Figure S57. a) Molecular structure, b) optimized structure, c) frontier molecular orbitals and corresponding energy levels, and calculated $\Delta \mathrm{E}$ of B55 in the ground state in acetonitrile at M062X/Def2SVP level.

a<smiles></smiles>

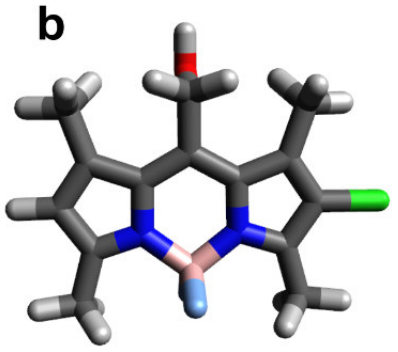

C<smiles>[AlH2]O[GeH3]</smiles>
$1.666 \mathrm{eV}$

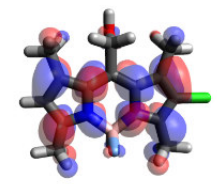

$-2.139 \mathrm{eV}$
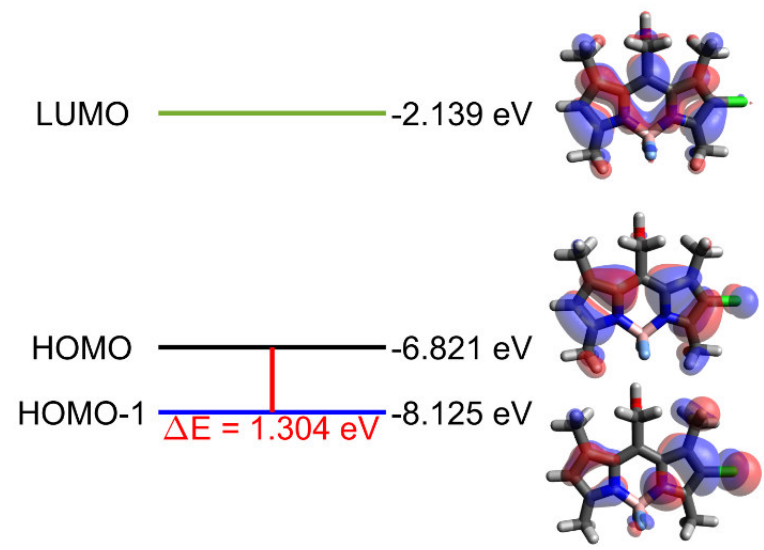

Figure S58. a) Molecular structure, b) optimized structure, c) frontier molecular orbitals and corresponding energy levels, and calculated $\Delta \mathrm{E}$ of B56 in the ground state in acetonitrile at M062X/Def2SVP level. 

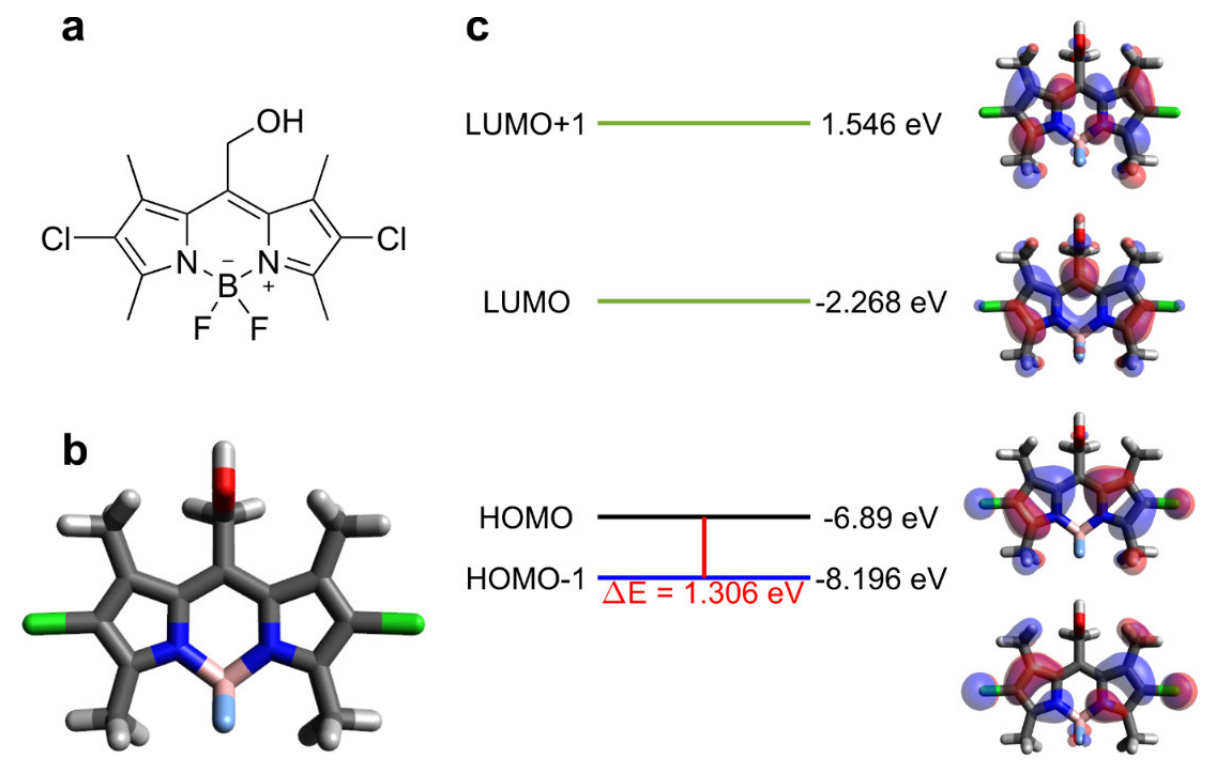

Figure S59. a) Molecular structure, b) optimized structure, c) frontier molecular orbitals and corresponding energy levels, and calculated $\Delta \mathrm{E}$ of B57 in the ground state in acetonitrile at M062X/Def2SVP level.

a<smiles></smiles>

C

LUMO+1 $0.728 \mathrm{eV}$

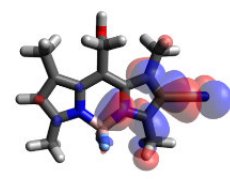

LUMO $-2.241 \mathrm{eV}$
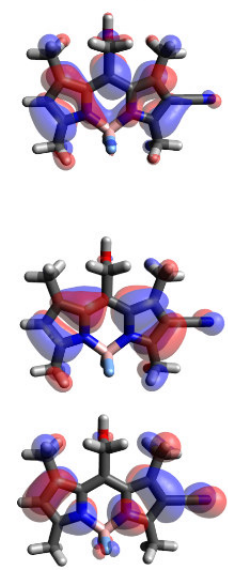

Figure S60. a) Molecular structure, b) optimized structure, c) frontier molecular orbitals and corresponding energy levels, and calculated $\Delta \mathrm{E}$ of B58 in the ground state in acetonitrile at M062X/Def2SVP level. 
<smiles></smiles>
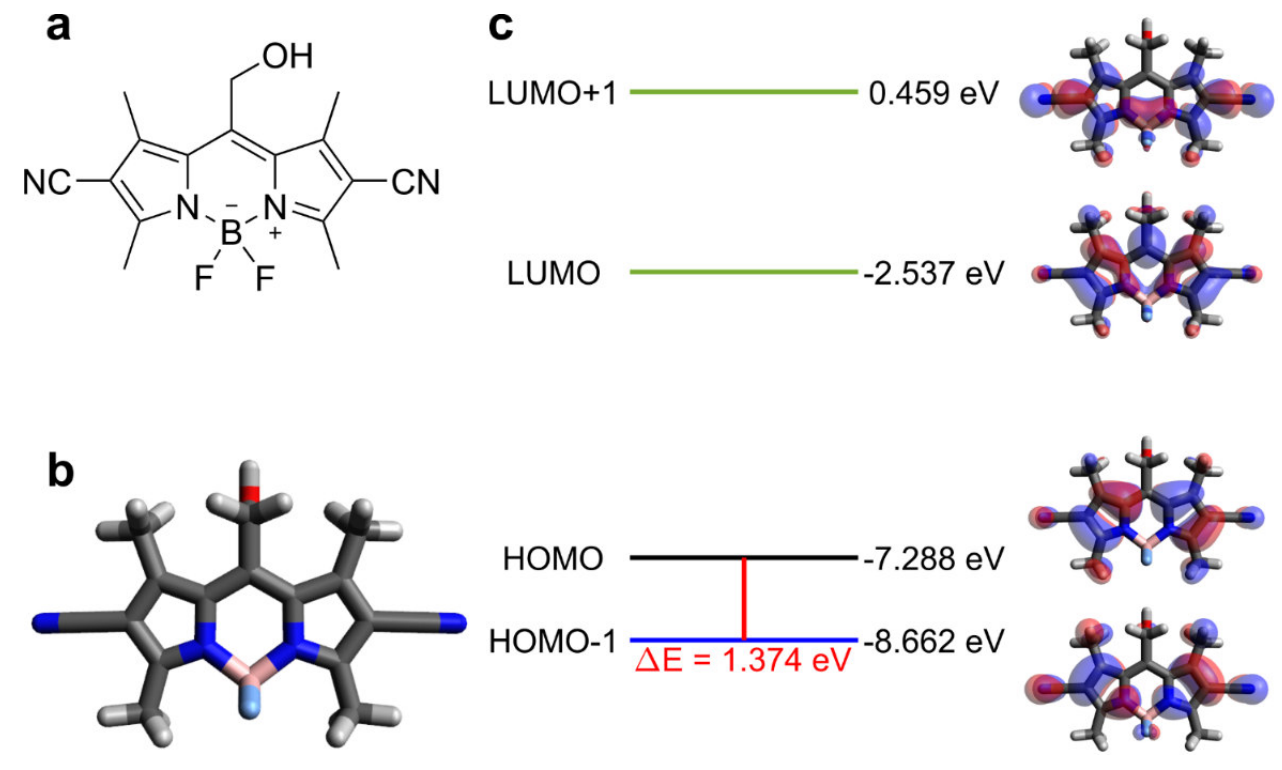

Figure S61. a) Molecular structure, b) optimized structure, c) frontier molecular orbitals and corresponding energy levels, and calculated $\Delta \mathrm{E}$ of B59 in the ground state in acetonitrile at M062X/Def2SVP level.

a<smiles></smiles>

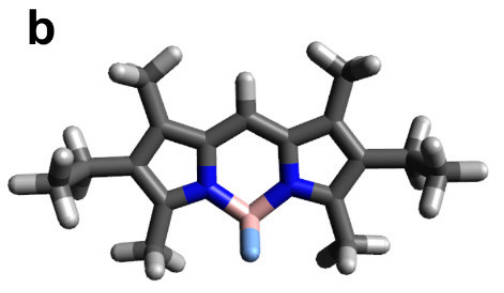

C

LUMO+1 $1.941 \mathrm{eV}$

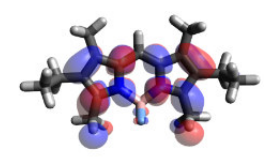

LUMO

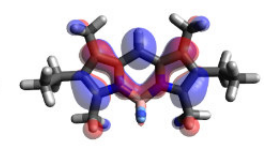

$-1.845 \mathrm{eV}$

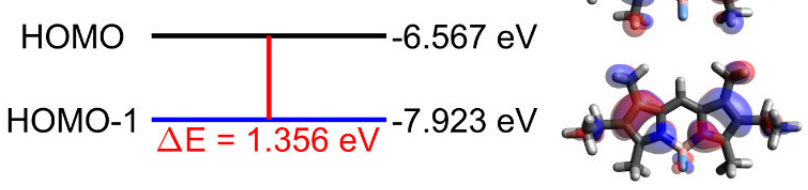

Figure S62. a) Molecular structure, b) optimized structure, c) frontier molecular orbitals and corresponding energy levels, and calculated $\Delta \mathrm{E}$ of $\mathrm{B} 60$ in the ground state in acetonitrile at M062X/Def2SVP level. 
a<smiles>CC1=CC(C)=[N+]2C1=Cc1c(C)cc(C)n1[B-]2(F)F</smiles>

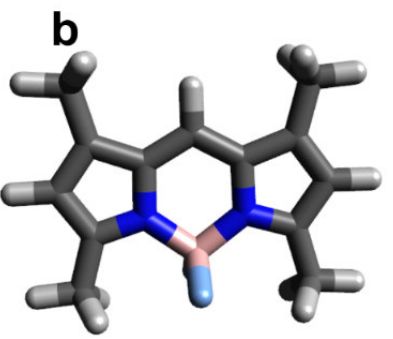

C

LUMO+1 $1.797 \mathrm{eV}$

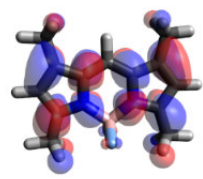

LUMO

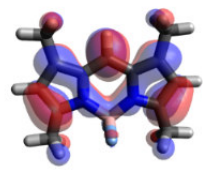

$-1.958 \mathrm{eV}$

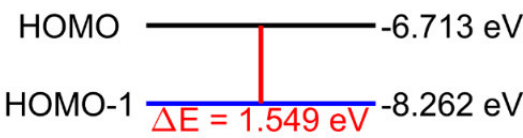

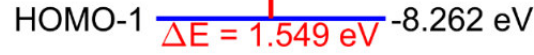

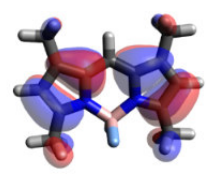

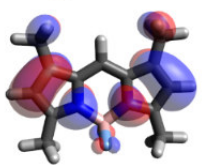

Figure S63. a) Molecular structure, b) optimized structure, c) frontier molecular orbitals and corresponding energy levels, and calculated $\Delta \mathrm{E}$ of B61 in the ground state in acetonitrile at M062X/Def2SVP level.

a<smiles>CC1=C(Cl)C(C)=[N+]2C1=Cc1c(C)cc(C)n1[B-]2(F)F</smiles>

$1.737 \mathrm{eV}$

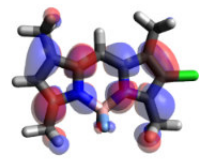

$-2.05 \mathrm{eV}$
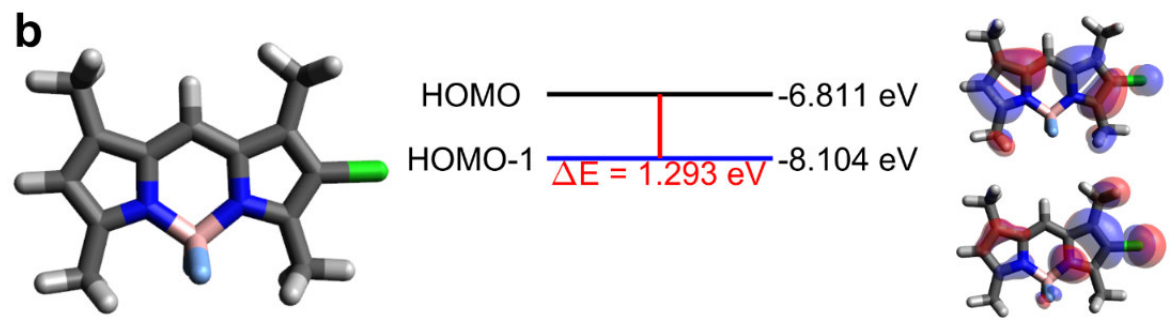

Figure S64. a) Molecular structure, b) optimized structure, c) frontier molecular orbitals and corresponding energy levels, and calculated $\Delta \mathrm{E}$ of B62 in the ground state in acetonitrile at M062X/Def2SVP level. 
<smiles></smiles>
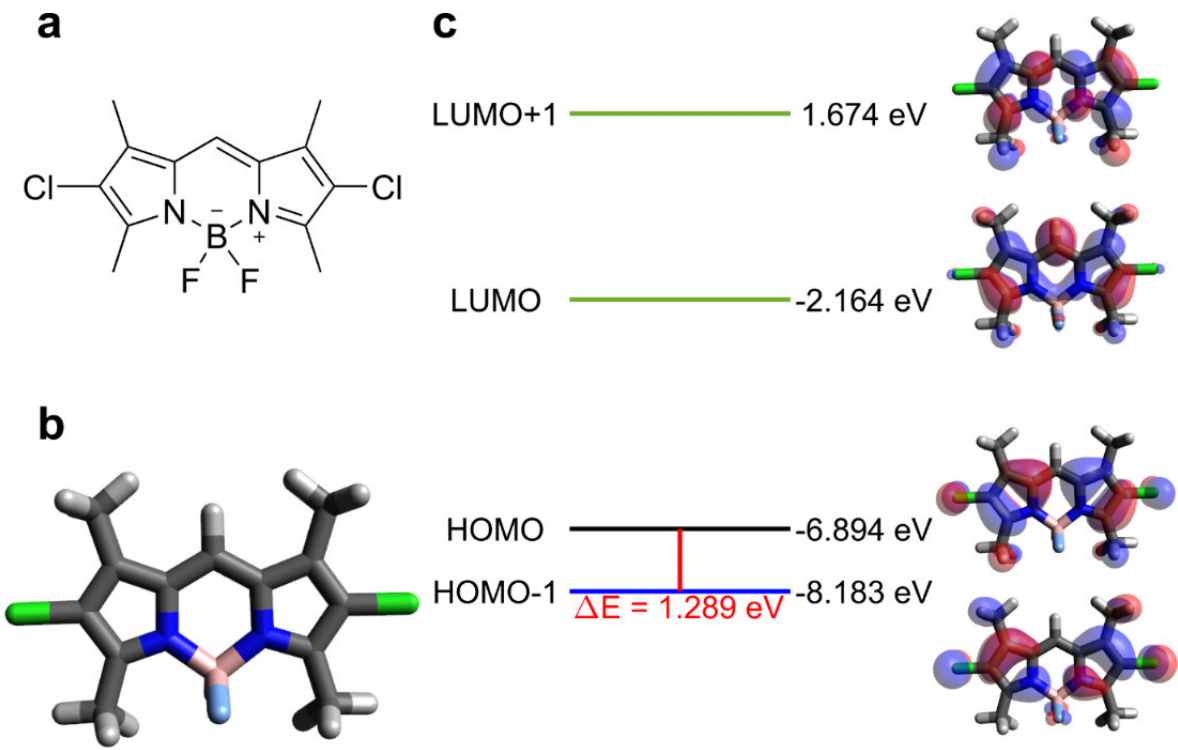

Figure S65. a) Molecular structure, b) optimized structure, c) frontier molecular orbitals and corresponding energy levels, and calculated $\Delta \mathrm{E}$ of B63 in the ground state in acetonitrile at M062X/Def2SVP level.
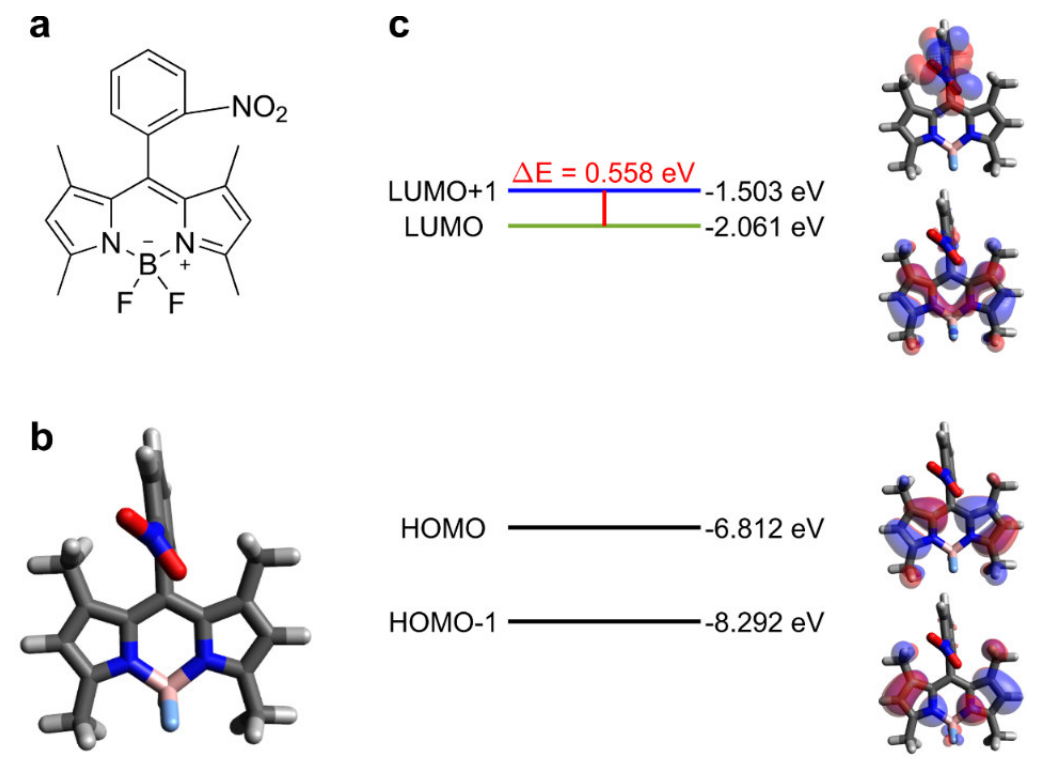

Figure S66. a) Molecular structure, b) optimized structure, c) frontier molecular orbitals and corresponding energy levels, and calculated $\triangle \mathrm{E}$ of B64 in the ground state in methanol at M062X/Def2SVP level. 
C
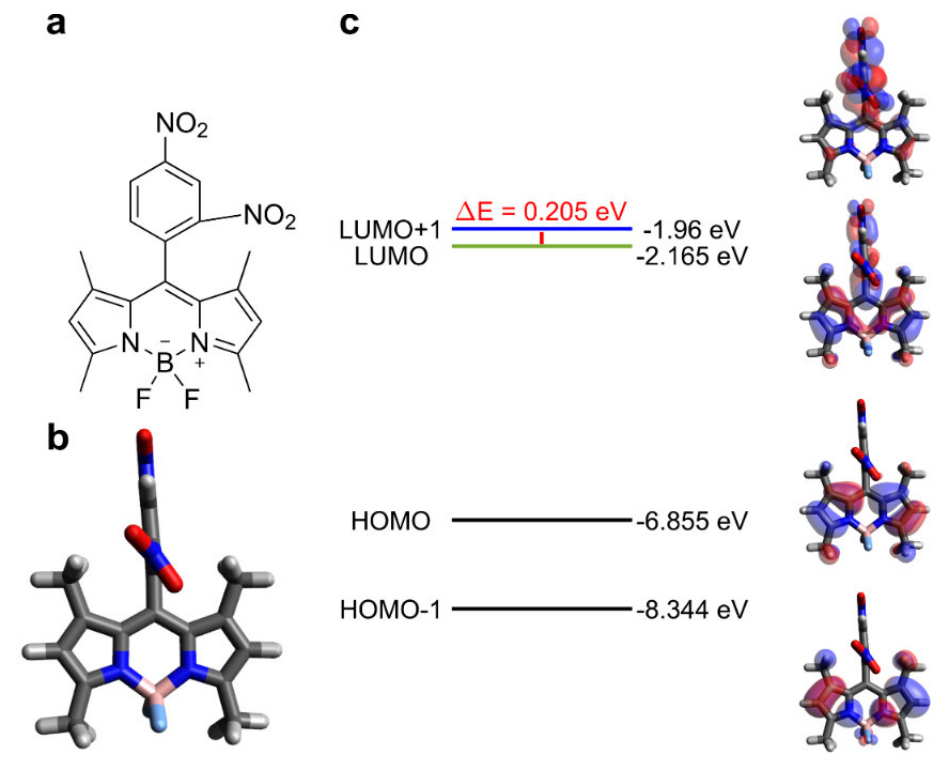

Figure S67. a) Molecular structure, b) optimized structure, c) frontier molecular orbitals and corresponding energy levels, and calculated $\Delta \mathrm{E}$ of $\mathrm{B} 65$ in the ground state in methanol at M062X/Def2SVP level.

a<smiles></smiles>

b

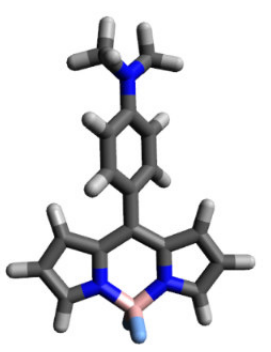

C
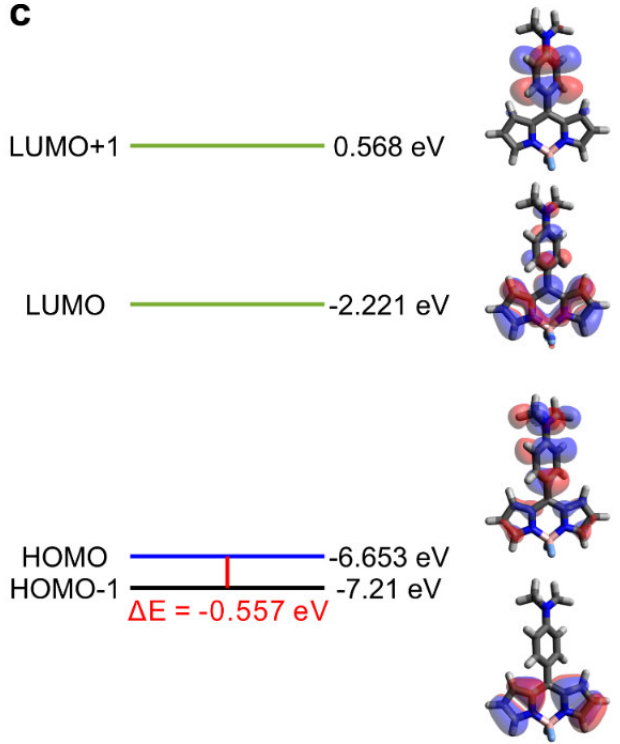

Figure S68. a) Molecular structure, b) optimized structure, c) frontier molecular orbitals and corresponding energy levels, and calculated $\triangle \mathrm{E}$ of B66 in the ground state in methanol at M062X/Def2SVP level. 
a
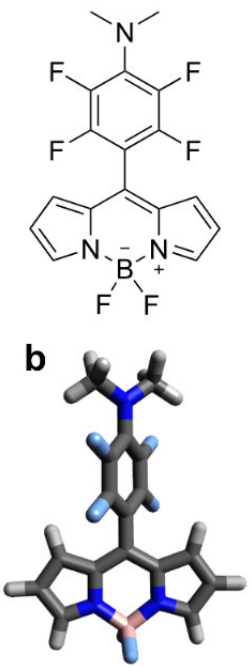

C

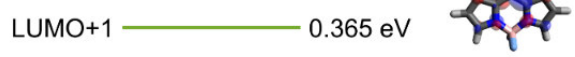

LUMO
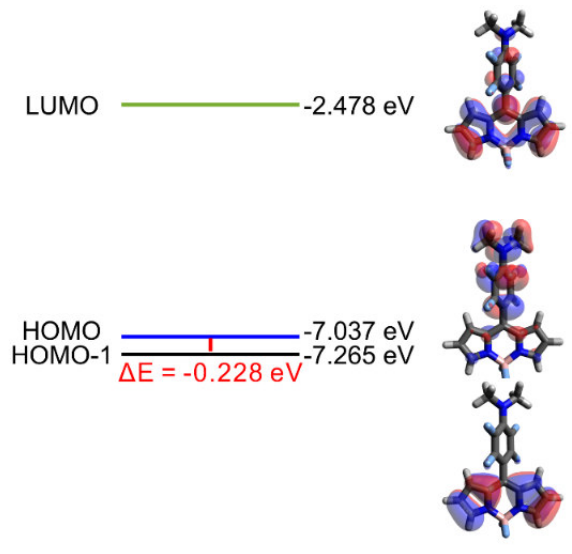

Figure S69. a) Molecular structure, b) optimized structure, c) frontier molecular orbitals and corresponding energy levels, and calculated $\Delta \mathrm{E}$ of $\mathrm{B} 67$ in the ground state in methanol at M062X/Def2SVP level.

a<smiles></smiles>

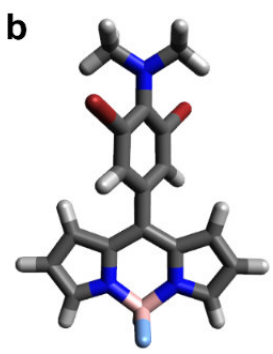

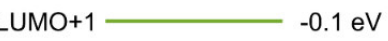

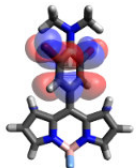

LUMO
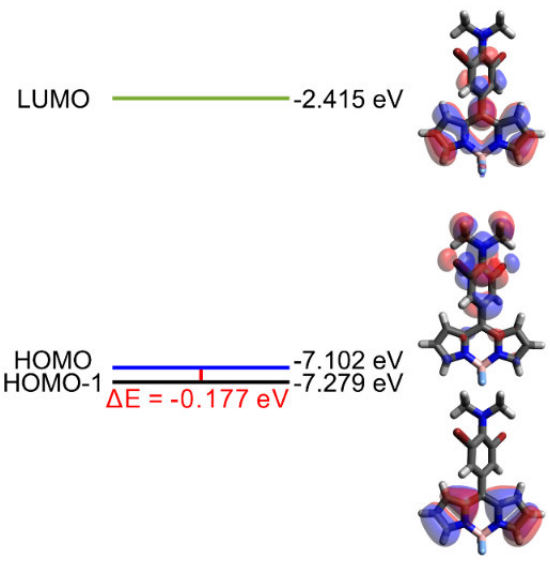

Figure S70. a) Molecular structure, b) optimized structure, c) frontier molecular orbitals and corresponding energy levels, and calculated $\Delta \mathrm{E}$ of $\mathbf{B 6 8}$ in the ground state in methanol at M062X/Def2SVP level. 
a<smiles></smiles>

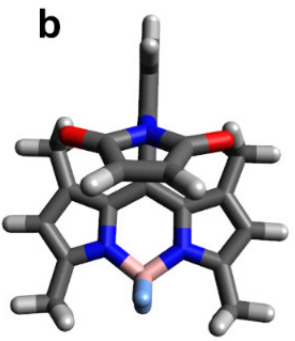

C
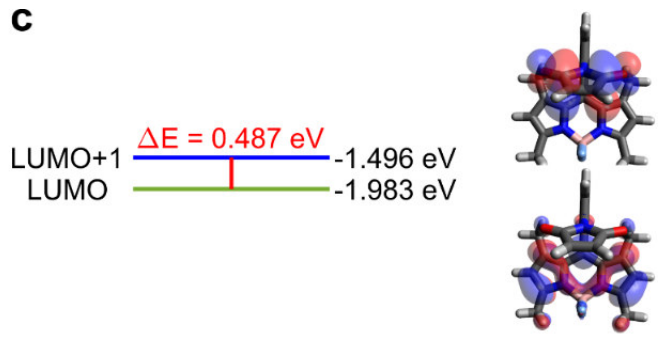

HOMO $-6.763 \mathrm{eV}$

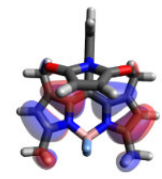

HOMO-1

$-8.213 \mathrm{eV}$

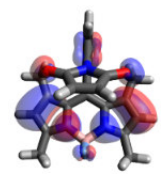

Figure S71. a) Molecular structure, b) optimized structure, c) frontier molecular orbitals and corresponding energy levels, and calculated $\Delta \mathrm{E}$ of B69 in the ground state in DMSO at M062X/Def2SVP level.

a

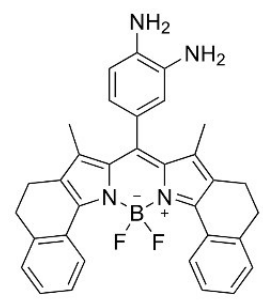

C

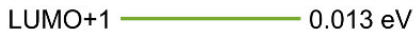

LUMO $-2.229 \mathrm{eV}$

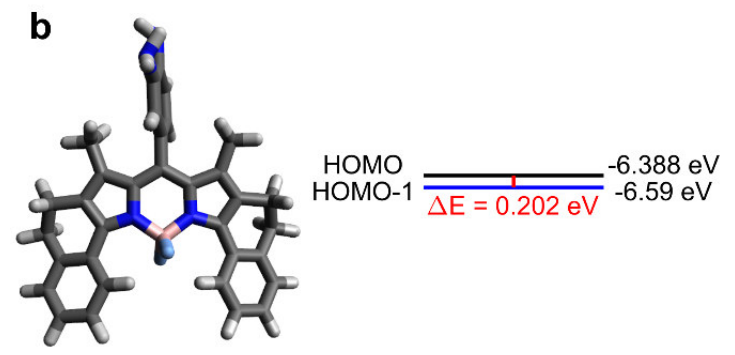

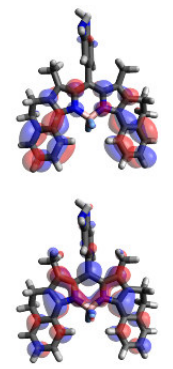

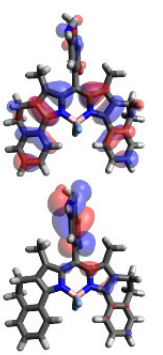

Figure S72. a) Molecular structure, b) optimized structure, c) frontier molecular orbitals and corresponding energy levels, and calculated $\Delta \mathrm{E}$ of B70 in the ground state in water at M062X/Def2SVP level. 

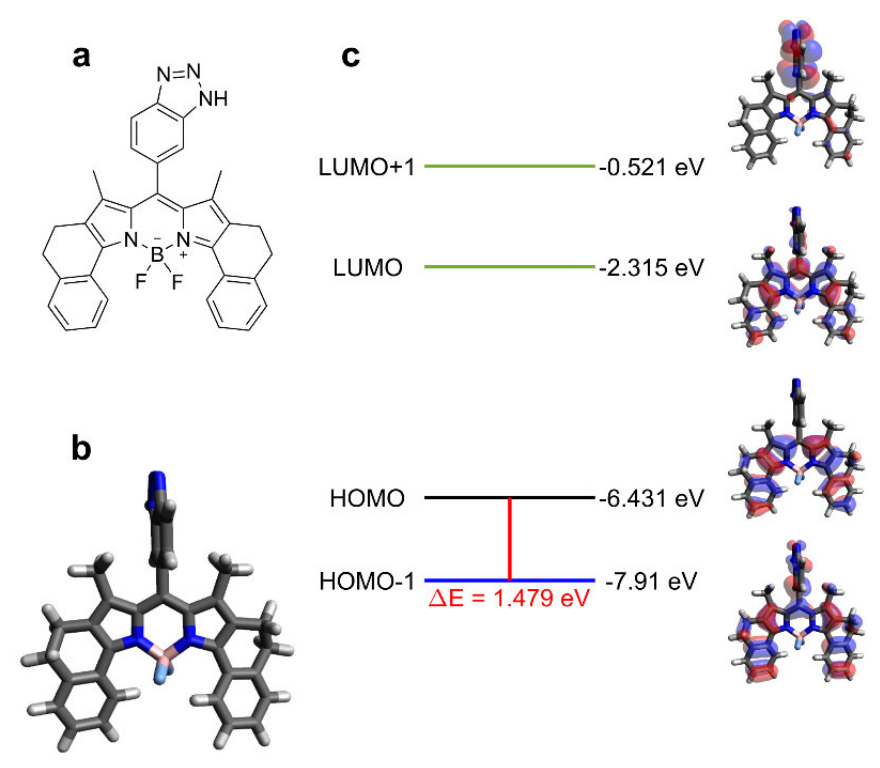

Figure S73. a) Molecular structure, b) optimized structure, c) frontier molecular orbitals and corresponding energy levels, and calculated $\Delta \mathrm{E}$ of B71 in the ground state in water at M062X/Def2SVP level.
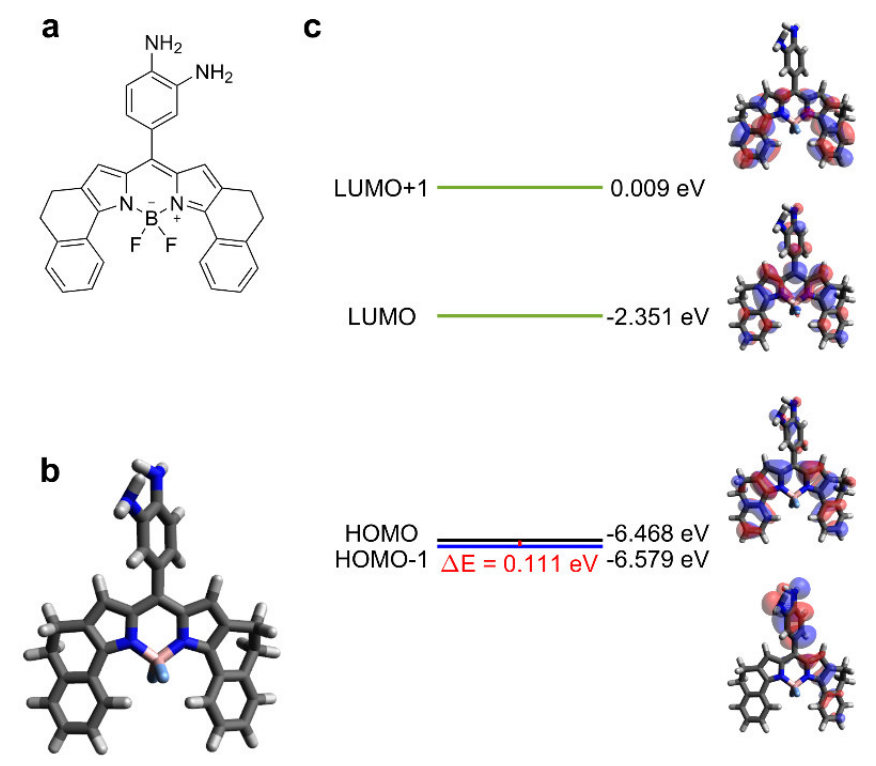

Figure S74. a) Molecular structure, b) optimized structure, c) frontier molecular orbitals and corresponding energy levels, and calculated $\Delta \mathrm{E}$ of B72 in the ground state in water at M062X/Def2SVP level. 

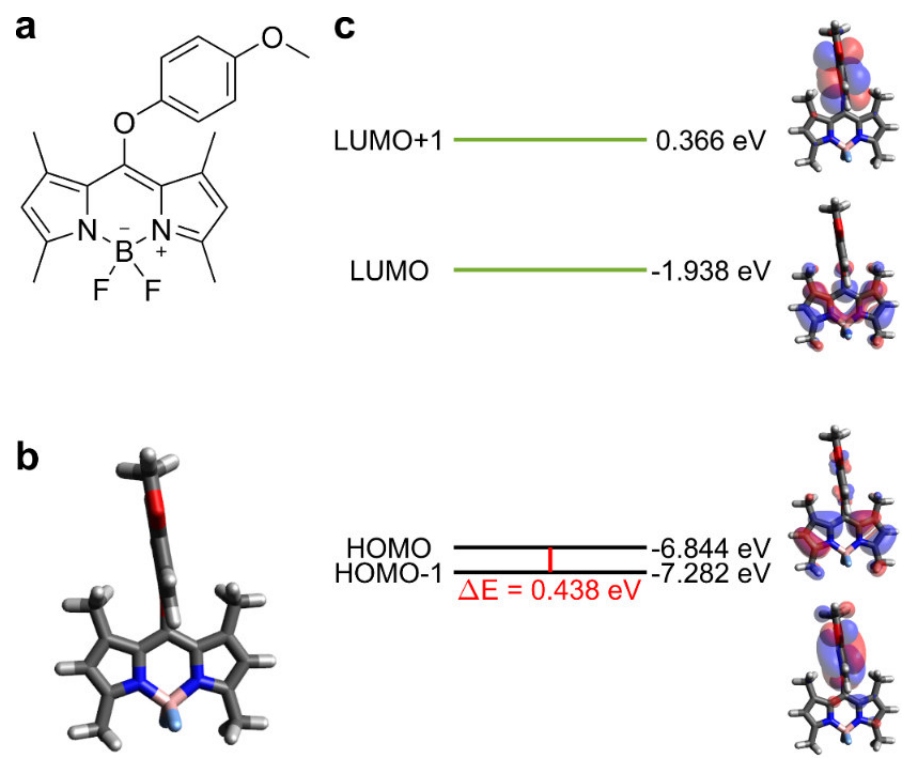

Figure S75. a) Molecular structure, b) optimized structure, c) frontier molecular orbitals and corresponding energy levels, and calculated $\triangle \mathrm{E}$ of B73 in the ground state in acetonitrile at M062X/Def2SVP level.

a<smiles></smiles>

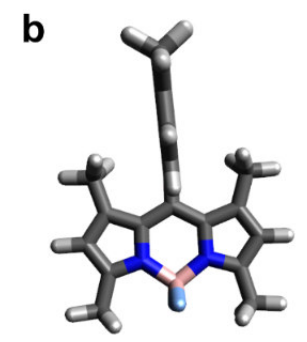

C

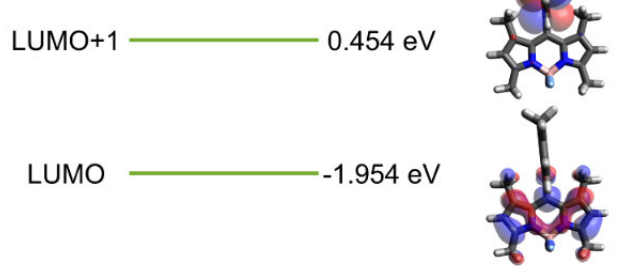

HOMO
HOMO-1
$\frac{\Delta E=0.854 \mathrm{eV}}{\Delta \mathrm{E}=}-7.706 \mathrm{eV}$

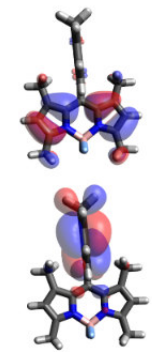

Figure S76. a) Molecular structure, b) optimized structure, c) frontier molecular orbitals and corresponding energy levels, and calculated $\Delta \mathrm{E}$ of B74 in the ground state in acetonitrile at M062X/Def2SVP level. 
a<smiles></smiles>

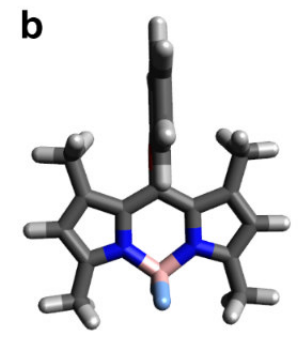

C

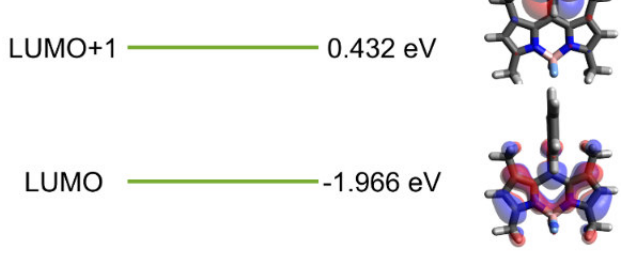

HOMO $-6.861 \mathrm{eV}$
HOMO-1 $\frac{1.124 \mathrm{eV}}{\frac{\mathrm{E}=1.985 \mathrm{eV}}{}}$
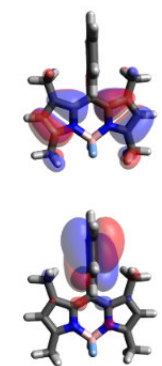

Figure S77. a) Molecular structure, b) optimized structure, c) frontier molecular orbitals and corresponding energy levels, and calculated $\triangle \mathrm{E}$ of B75 in the ground state in acetonitrile at M062X/Def2SVP level.

a<smiles></smiles>

b

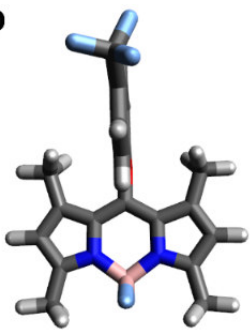

C
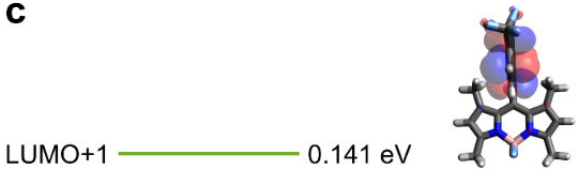

LUMO $-2.005 \mathrm{eV}$

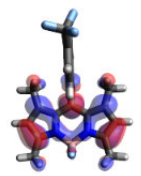

HOMO $-6.884 \mathrm{eV}$
HOMO-1 $\frac{}{\Delta E=1.397 \mathrm{eV}}-8.281 \mathrm{eV}$

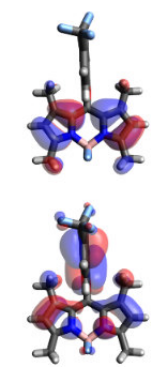

Figure S78. a) Molecular structure, b) optimized structure, c) frontier molecular orbitals and corresponding energy levels, and calculated $\Delta \mathrm{E}$ of $\mathbf{B 7 6}$ in the ground state in PBS at M062X/Def2SVP level. 
a<smiles></smiles>

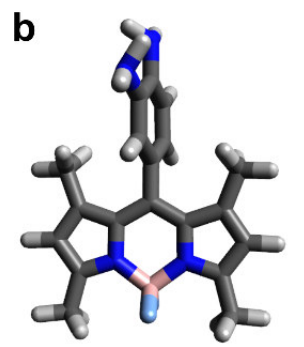

C
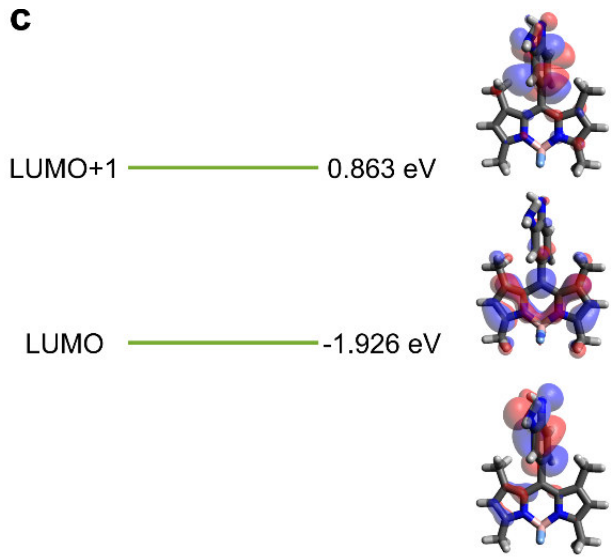

HOMO

$\frac{1}{\Delta E=-0.19 \mathrm{eV}}-6.567 \mathrm{eV}$

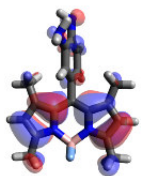

Figure S79. a) Molecular structure, b) optimized structure, c) frontier molecular orbitals and corresponding energy levels, and calculated $\triangle \mathrm{E}$ of $\mathrm{B} 77$ in the ground state in acetonitrile at M062X/Def2SVP level.<smiles></smiles>

b

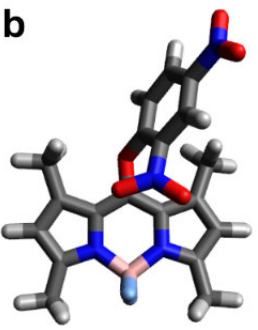

C
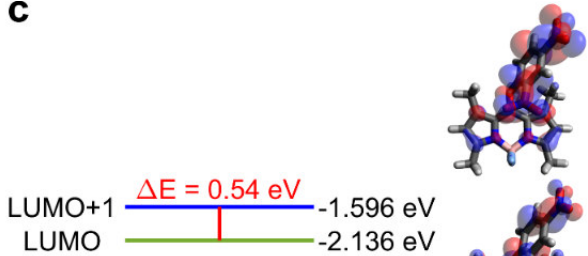
$136 \mathrm{eV}$
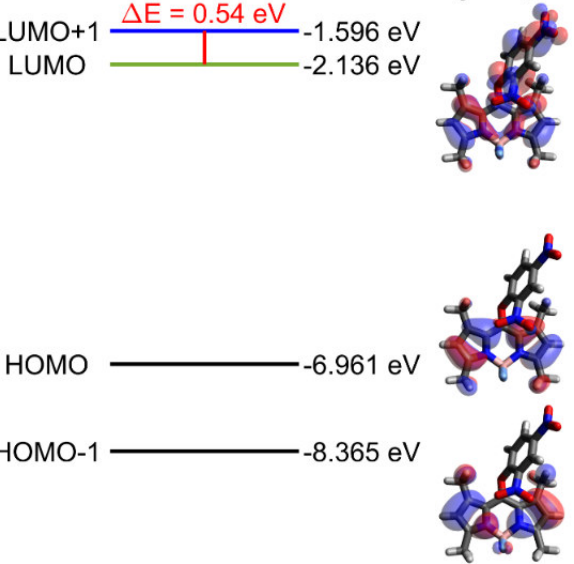

Figure S80. a) Molecular structure, b) optimized structure, c) frontier molecular orbitals and corresponding energy levels, and calculated $\Delta \mathrm{E}$ of $\mathrm{B78}$ in the ground state in acetonitrile at M062X/Def2SVP level. 
a

C<smiles></smiles>

LUMO+1 $0.381 \mathrm{eV}$

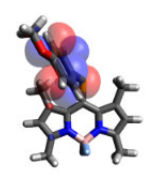

LUMO $-2.183 \mathrm{eV}$

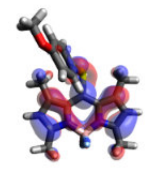

b

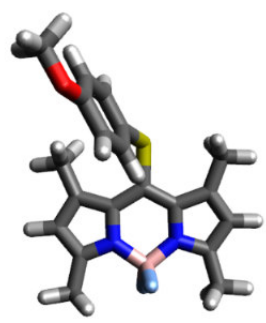

HOMO $-6.838 \mathrm{eV}$

HOMO-1 $\frac{1}{\Delta E=0.251 \mathrm{eV}}-7.089 \mathrm{eV}$

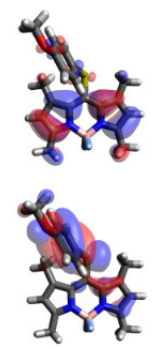

Figure S81. a) Molecular structure, b) optimized structure, c) frontier molecular orbitals and corresponding energy levels, and calculated $\triangle \mathrm{E}$ of B79 in the ground state in acetonitrile at M062X/Def2SVP level.

a

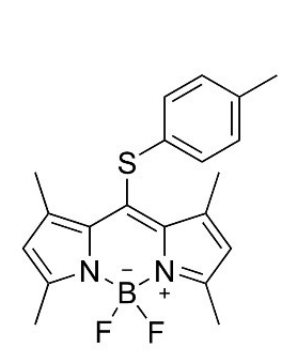

b

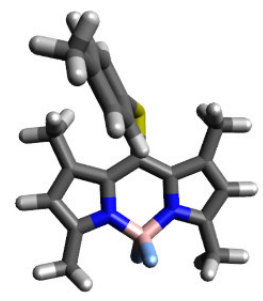

C

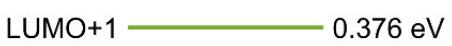

LUMO $-2.208 \mathrm{eV}$
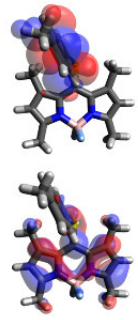

HOMO

$-6.842 \mathrm{eV}$

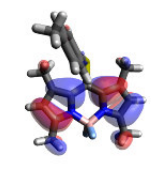

HOMO-1 $\frac{1}{\Delta \mathrm{E}=0.506 \mathrm{eV}}-7.348 \mathrm{eV}$

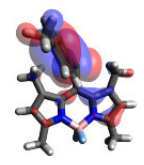

Figure S82. a) Molecular structure, b) optimized structure, c) frontier molecular orbitals and corresponding energy levels, and calculated $\Delta \mathrm{E}$ of $\mathbf{B 8 0}$ in the ground state in acetonitrile at M062X/Def2SVP level. 

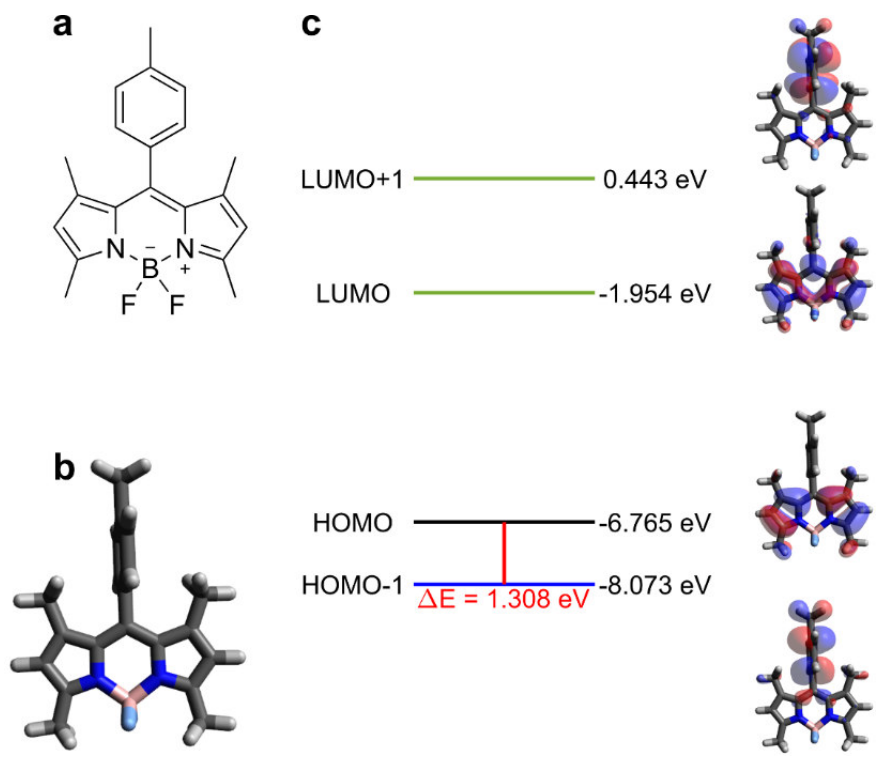

Figure S83. a) Molecular structure, b) optimized structure, c) frontier molecular orbitals and corresponding energy levels, and calculated $\Delta \mathrm{E}$ of $\mathrm{B} 81$ in the ground state in methanol at M062X/Def2SVP level.<smiles></smiles>
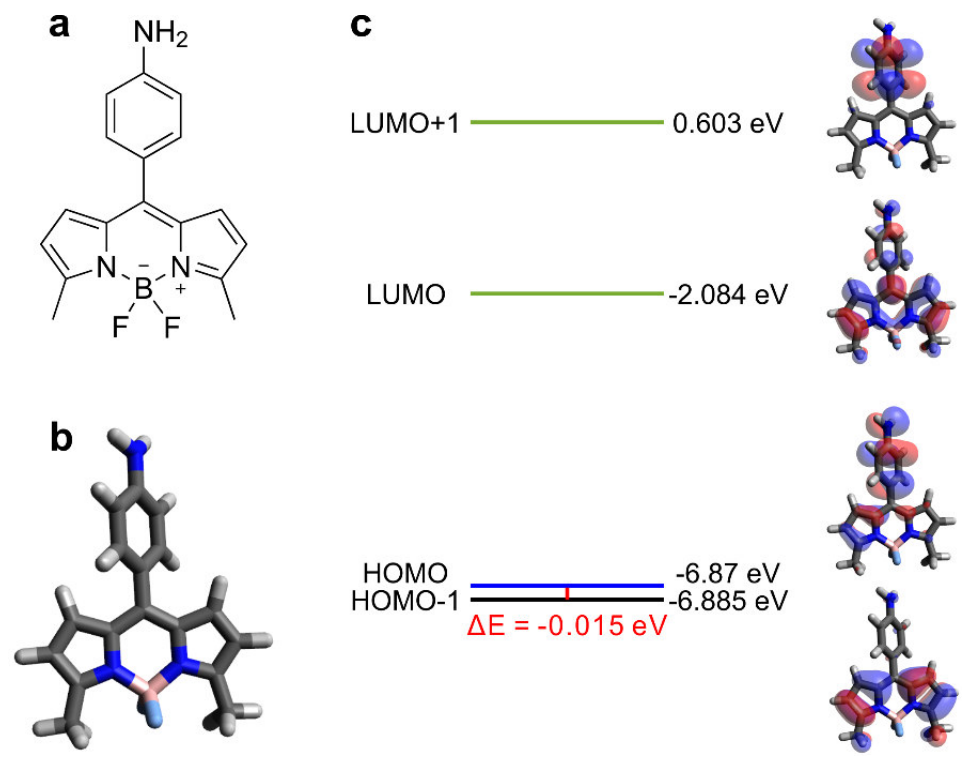

Figure S84. a) Molecular structure, b) optimized structure, c) frontier molecular orbitals and corresponding energy levels, and calculated $\Delta \mathrm{E}$ of $\mathrm{B} 82$ in the ground state in methanol at M062X/Def2SVP level. 

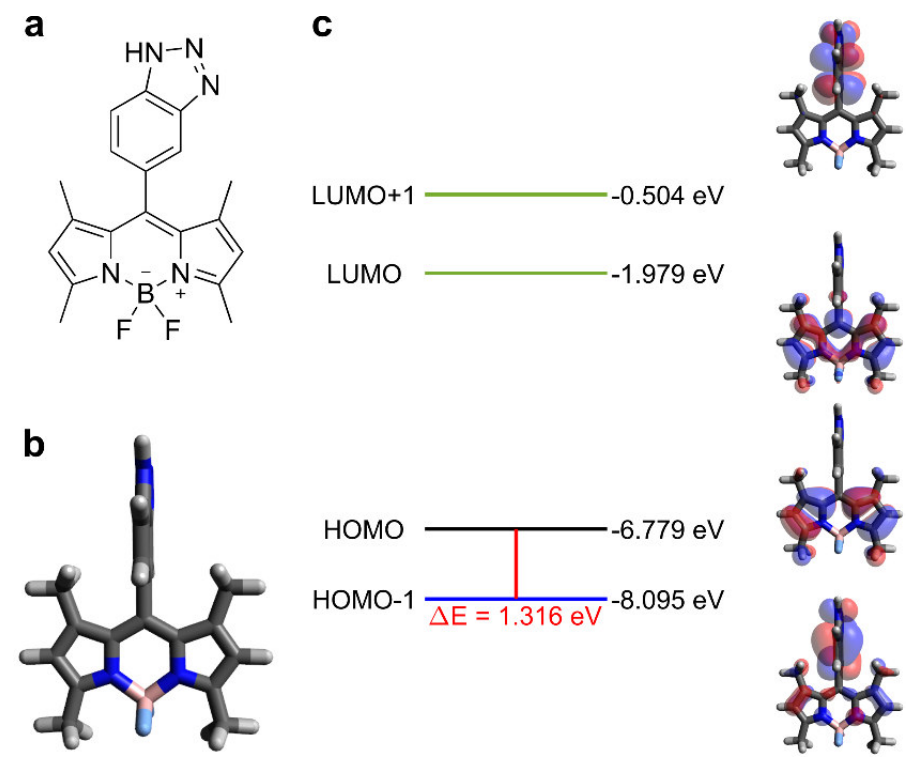

Figure S85. a) Molecular structure, b) optimized structure, c) frontier molecular orbitals and corresponding energy levels, and calculated $\triangle \mathrm{E}$ of B83 in the ground state in PBS at M062X/Def2SVP level.

a

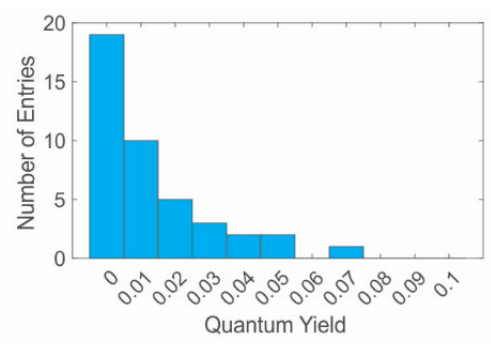

C

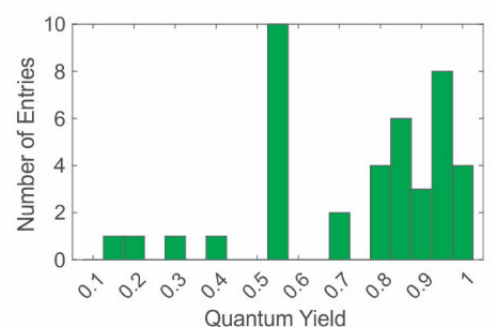

b

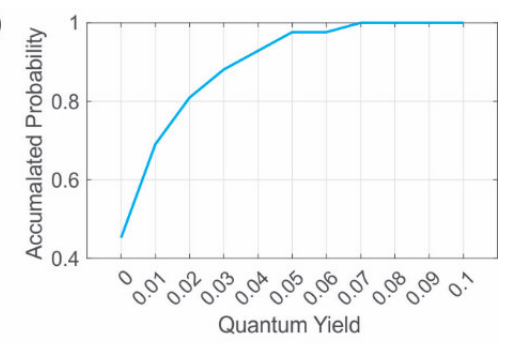

d

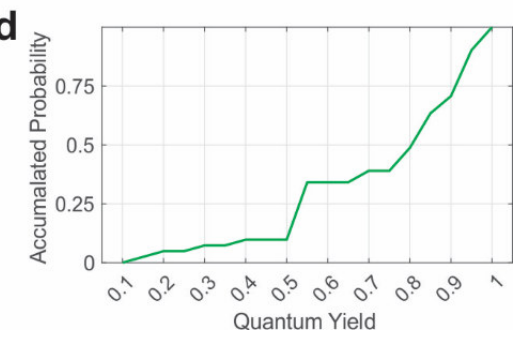

Figure S86. The histogram and accumulated probability of quantum yields for BODIPY dyes B1-B83 in polar solvents with $(\mathrm{a}, \mathrm{b}) \Delta \mathrm{E}<0.6 \mathrm{eV}$ and $(\mathrm{c}, \mathrm{d}) \Delta \mathrm{E}>0.6 \mathrm{eV}$. 


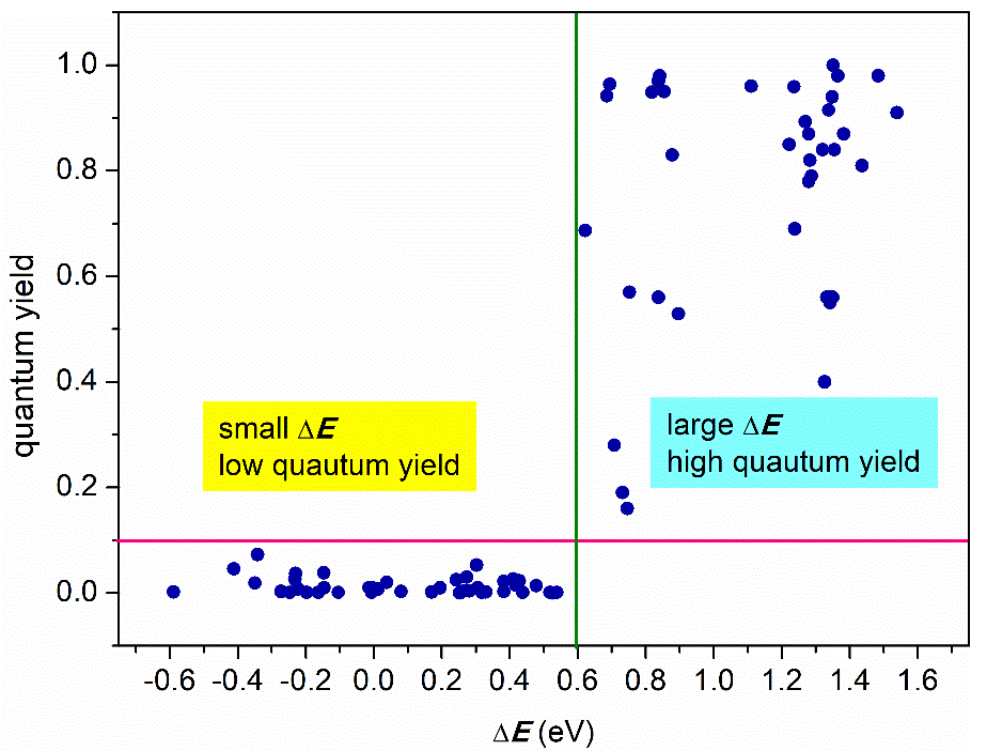

Figure S87. Correlation between quantum yields and calculated $\Delta E$ at $\omega B 97 X D / D e f 2 S V P$ level for mesophenyl substituted BODIPY compounds B1-B83 in polar solvents.

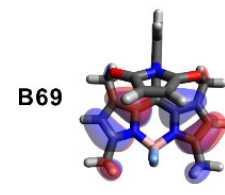

HOMO

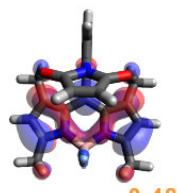

LUMO $\stackrel{0.487 \mathrm{eV}}{\longleftrightarrow} \mathrm{LUMO}+1$
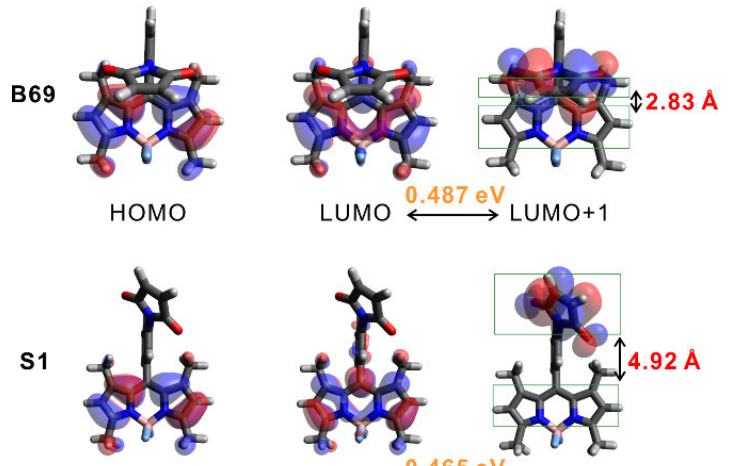

$\mathrm{HOMO}$
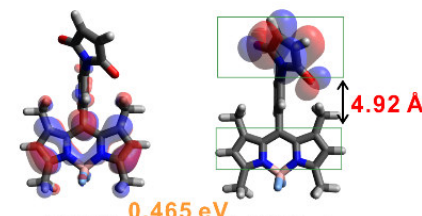

LUMO $\stackrel{0.465 \mathrm{eV}}{\longleftrightarrow}$ LUMO+1

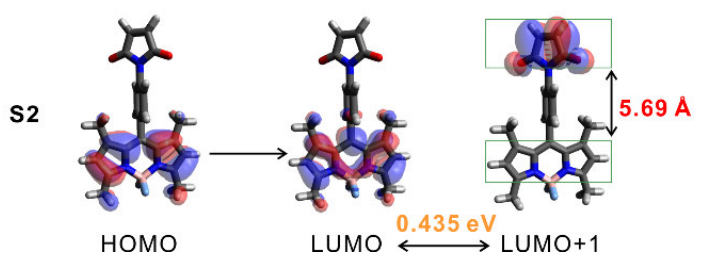

Figure S88. The frontier molecular orbitals and corresponding $\triangle \mathrm{E}$ of B69, S1, and S2, calculated in the ground state in DMSO at M062X/Def2SVP level. The distances between the fluorophore and the quencher moieties are highlighted in red.

Note: although S1 and S2 also have small $\Delta \mathrm{E}(0.465 \mathrm{eV}$ and $0.435 \mathrm{eV}$, respectively), their quantum yield are up to 0.37 and 0.54 in DMSO. This can be explained by the distance $\left(D_{\mathrm{f}-\mathrm{g}}\right)$ between the fluorescent core unit and the quenching moiety. Compared with B69, large $D_{\mathrm{t}-\mathrm{q}}$ values of S1 $(4.92 \AA)$ and S2 (5.69 $\AA$ ) reduce photoinduced electron transfer rates. The relationship between electron transfer rate and the distance between donor and acceptor fragments have been reported. ${ }^{35}$

From the structural point of view, $\mathbf{S} 1$ and $\mathbf{S 2}$ adopt the "fluorophore-spacer-receptor" format, while the phenyl ring is not involved in the frontier molecular orbitals and functions as a "spacer". This "spacer" effectively increased the distance between the BODIPY fluorophore and the quencher moieties.

In B69, the "spacer" is essentially bypassed, as the "quencher" and the fluorophore are at proximity to each other with a small $\mathrm{D}_{f-q}$ value. 
${ }^{1} \mathrm{H}-\mathrm{NMR}$ spectra of B1-B6 and ${ }^{1} \mathrm{H}-\mathrm{NMR},{ }^{13} \mathrm{C}-\mathrm{NMR}$, and HRMS of M1-M3.

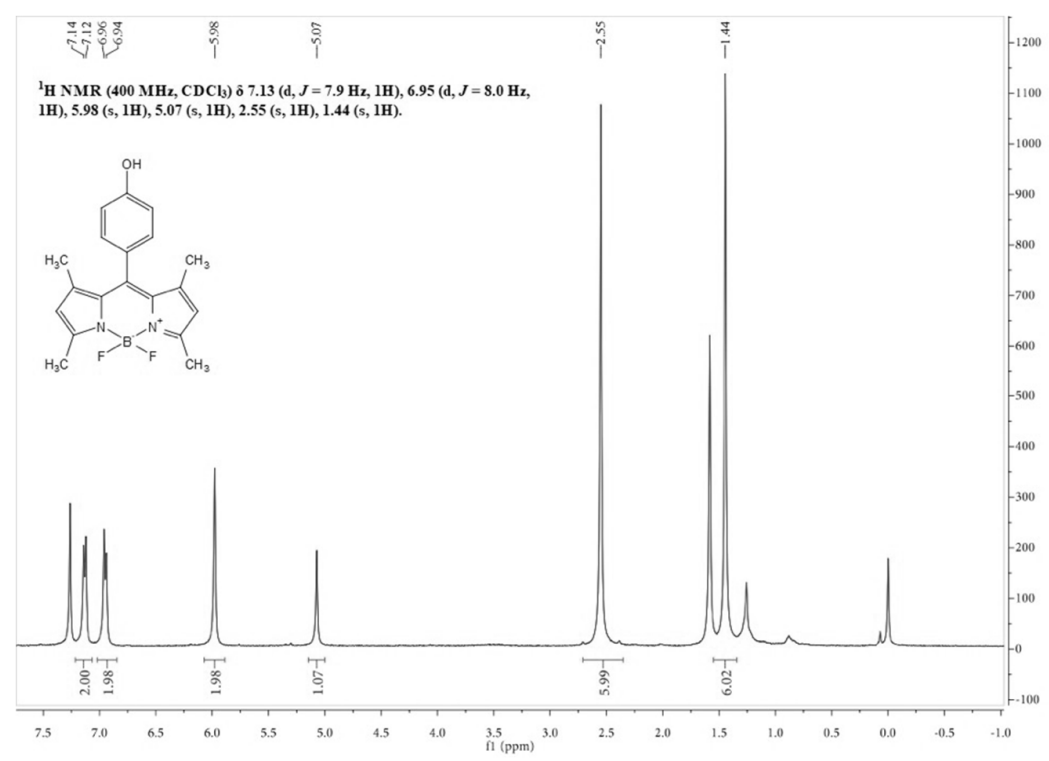

Figure S89. ${ }^{1} \mathrm{H}$ NMR spectrum of compound $\mathbf{B} 1$ in $\mathrm{CDCl}_{3}$.

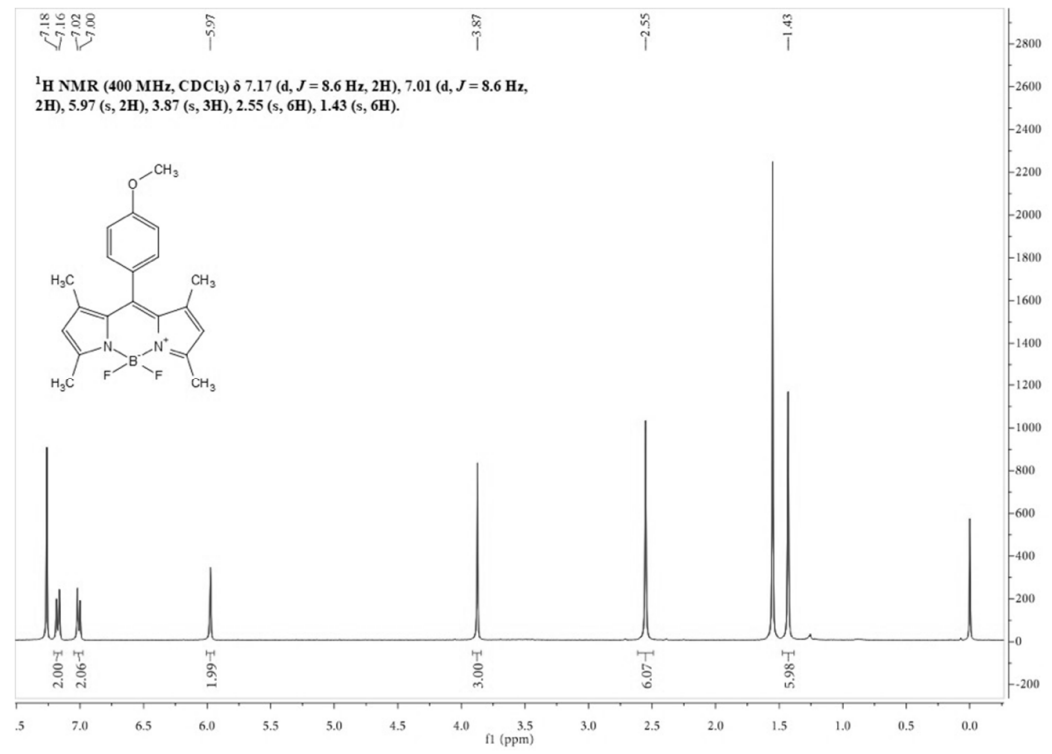

Figure S90. ${ }^{1} \mathrm{H}$ NMR spectrum of compound $\mathbf{B} 2$ in $\mathrm{CDCl}_{3}$. 


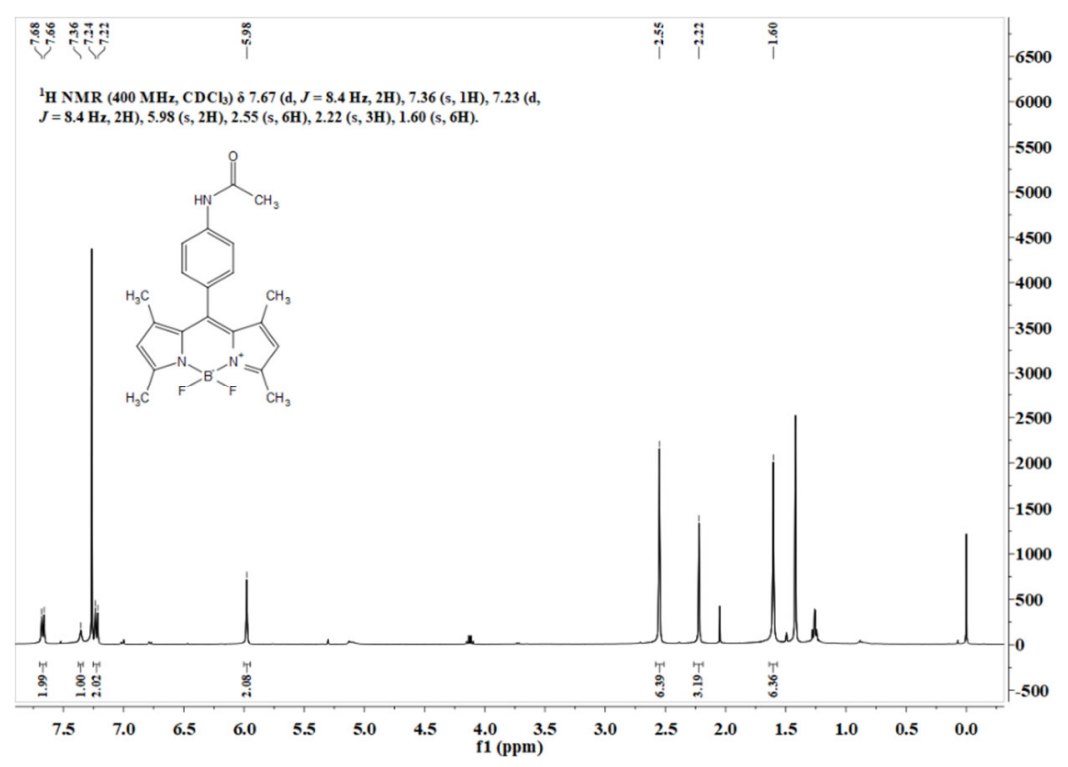

Figure S91. ${ }^{1} \mathrm{H}$ NMR spectrum of compound $\mathrm{B} 3$ in $\mathrm{CDCl}_{3}$.

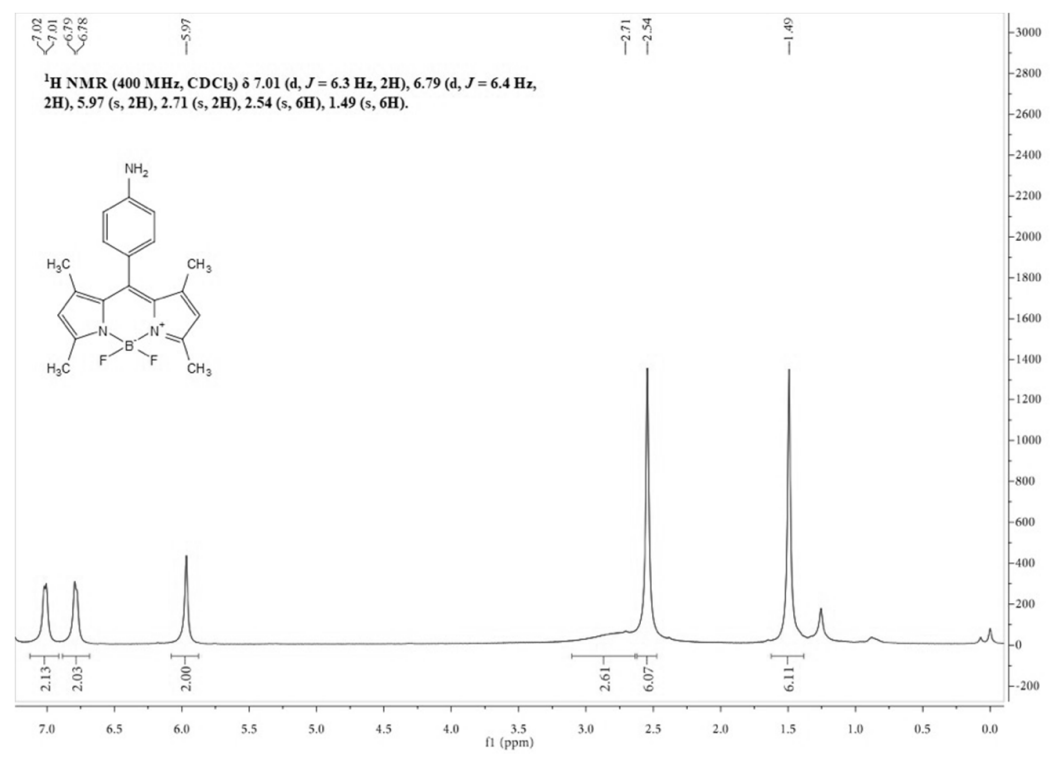

Figure S92. ${ }^{1} \mathrm{H}$ NMR spectrum of compound $\mathbf{B} 4$ in $\mathrm{CDCl}_{3}$. 


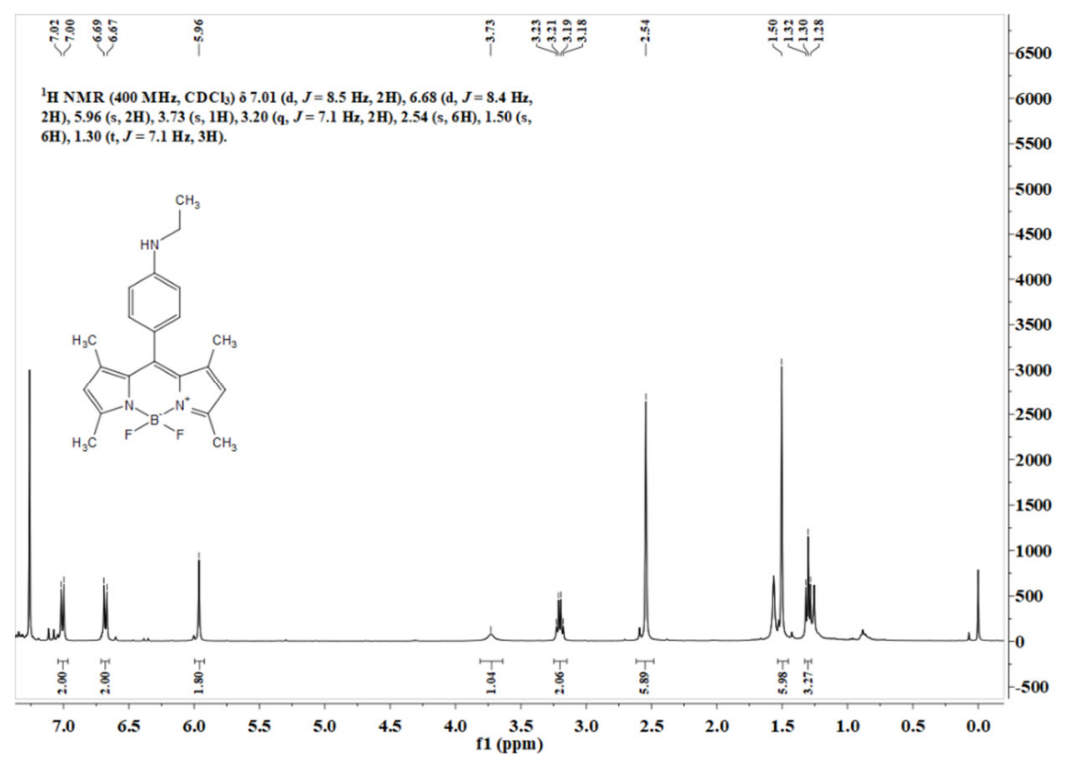

Figure S93. ${ }^{1} \mathrm{H}$ NMR spectrum of compound $\mathrm{B} 5$ in $\mathrm{CDCl}_{3}$.

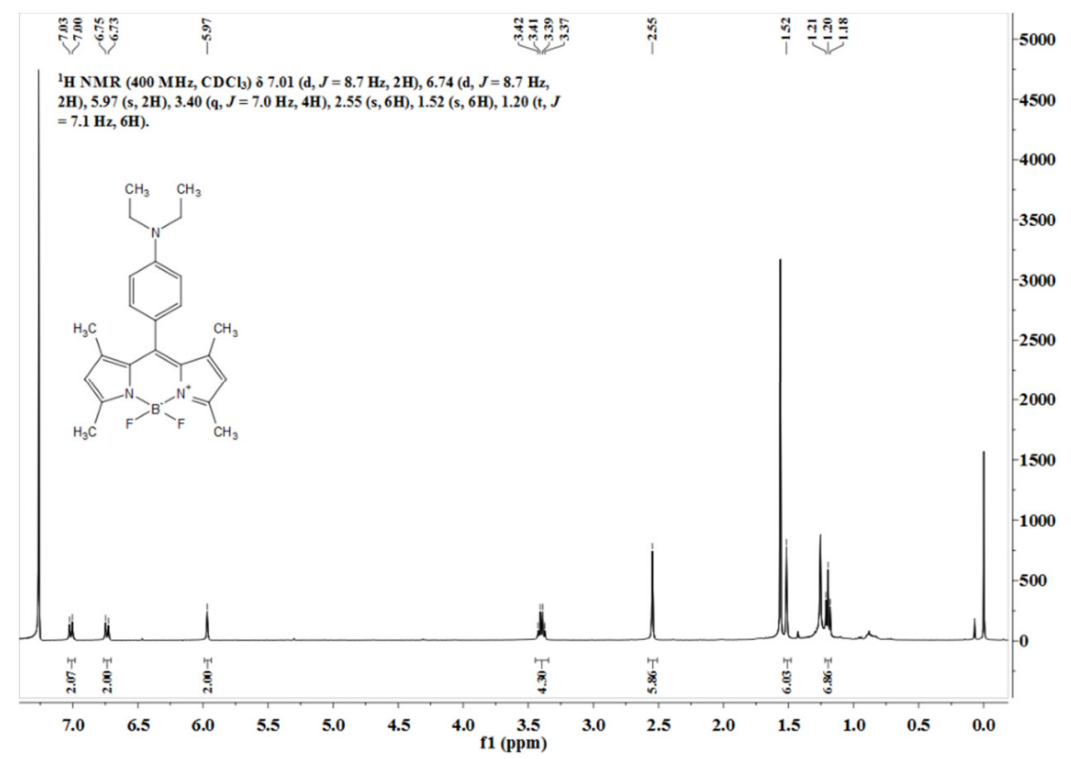

Figure S94. ${ }^{1} \mathrm{H}$ NMR spectrum of compound $\mathrm{B} 6$ in $\mathrm{CDCl}_{3}$. 


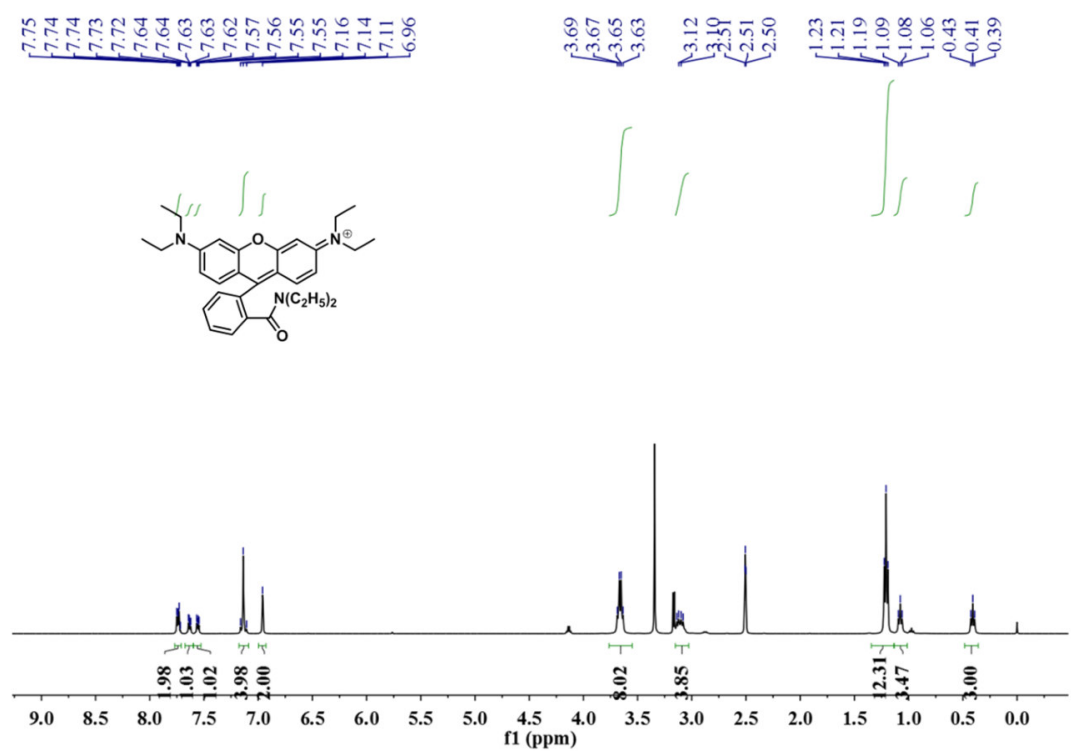

Figure S95. ${ }^{1} \mathrm{H}$ NMR spectrum of M1 in DMSO-d6.

${ }^{13}$ C NMR (101 MHz, DMSO) $\delta$ 167.52, 157.49, 156.01, 155.60, 137.09, 132.33, 130.70, 130.57, $130.35,129.74,126.66,114.58,113.54,96.28,49.05,45.84,43.08,37.72,14.11,12.92,11.33$.

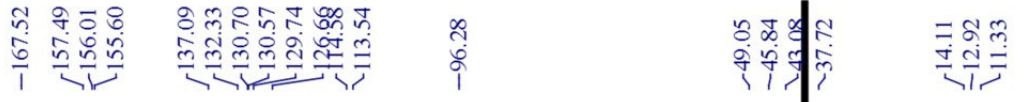
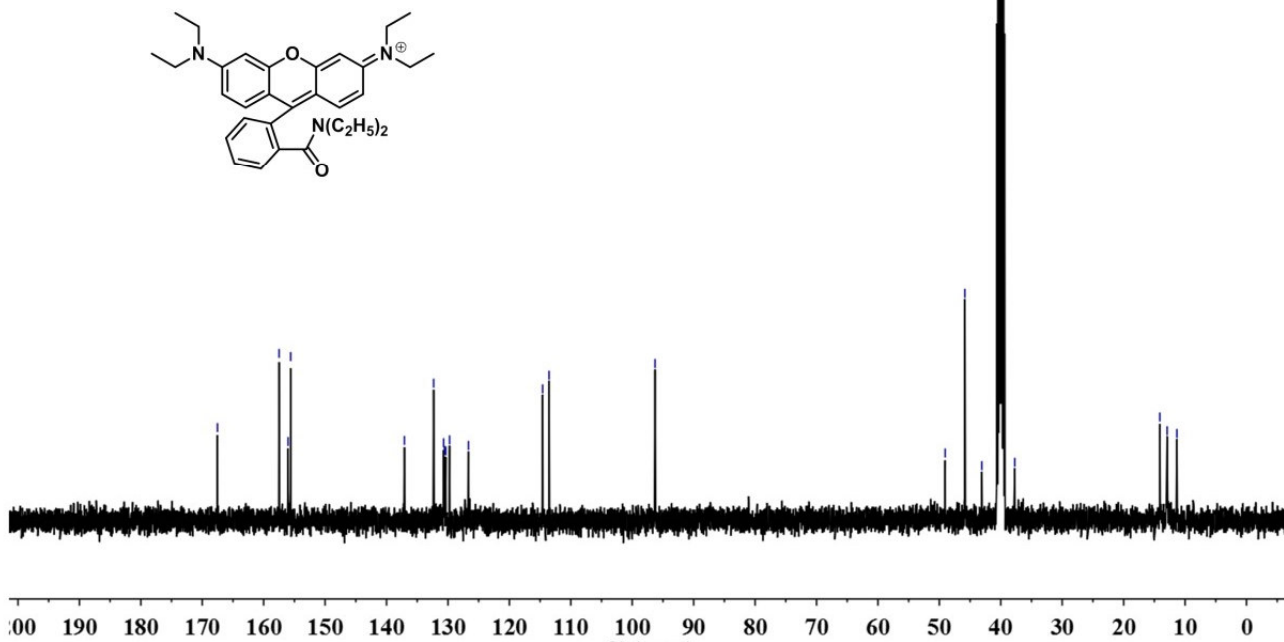

Figure S96. ${ }^{13} \mathrm{C}$ NMR spectrum of $\mathbf{M} 1$. 


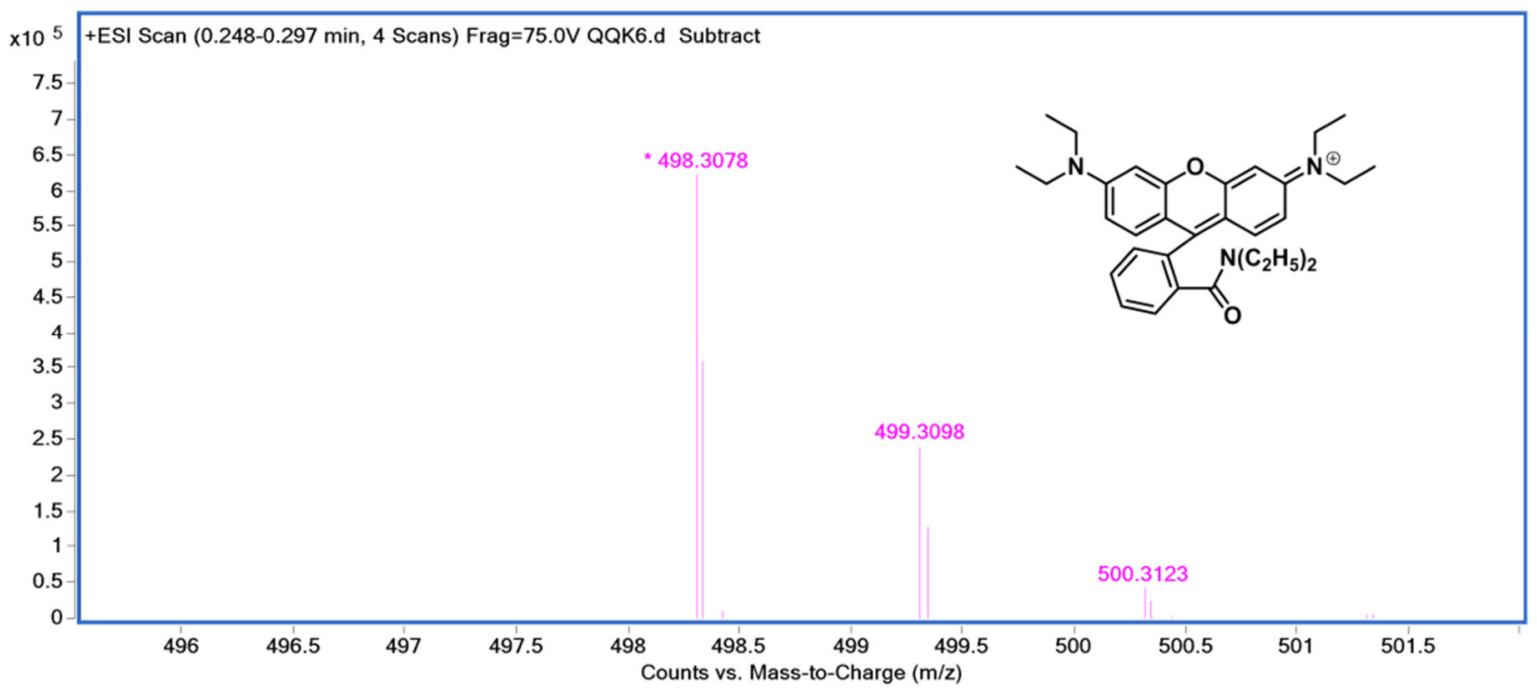

Figure S97. HRMS spectrum of M1.

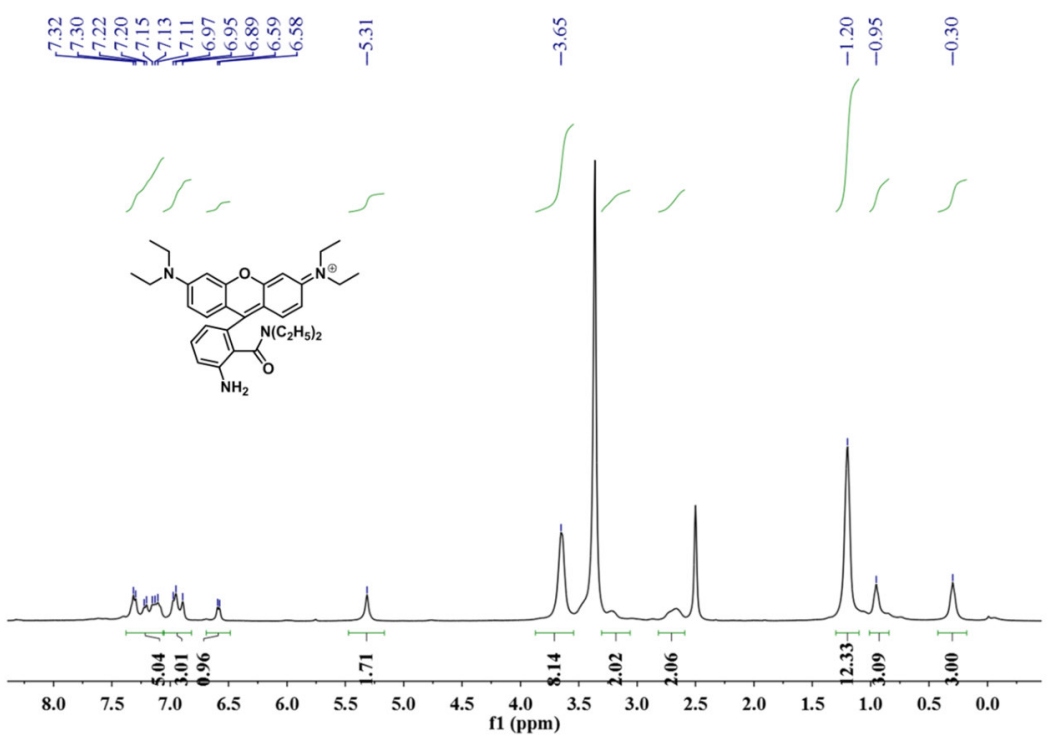

Figure S98. ${ }^{1} \mathrm{H}$ NMR spectrum of M2 in DMSO- $d_{6}$. 


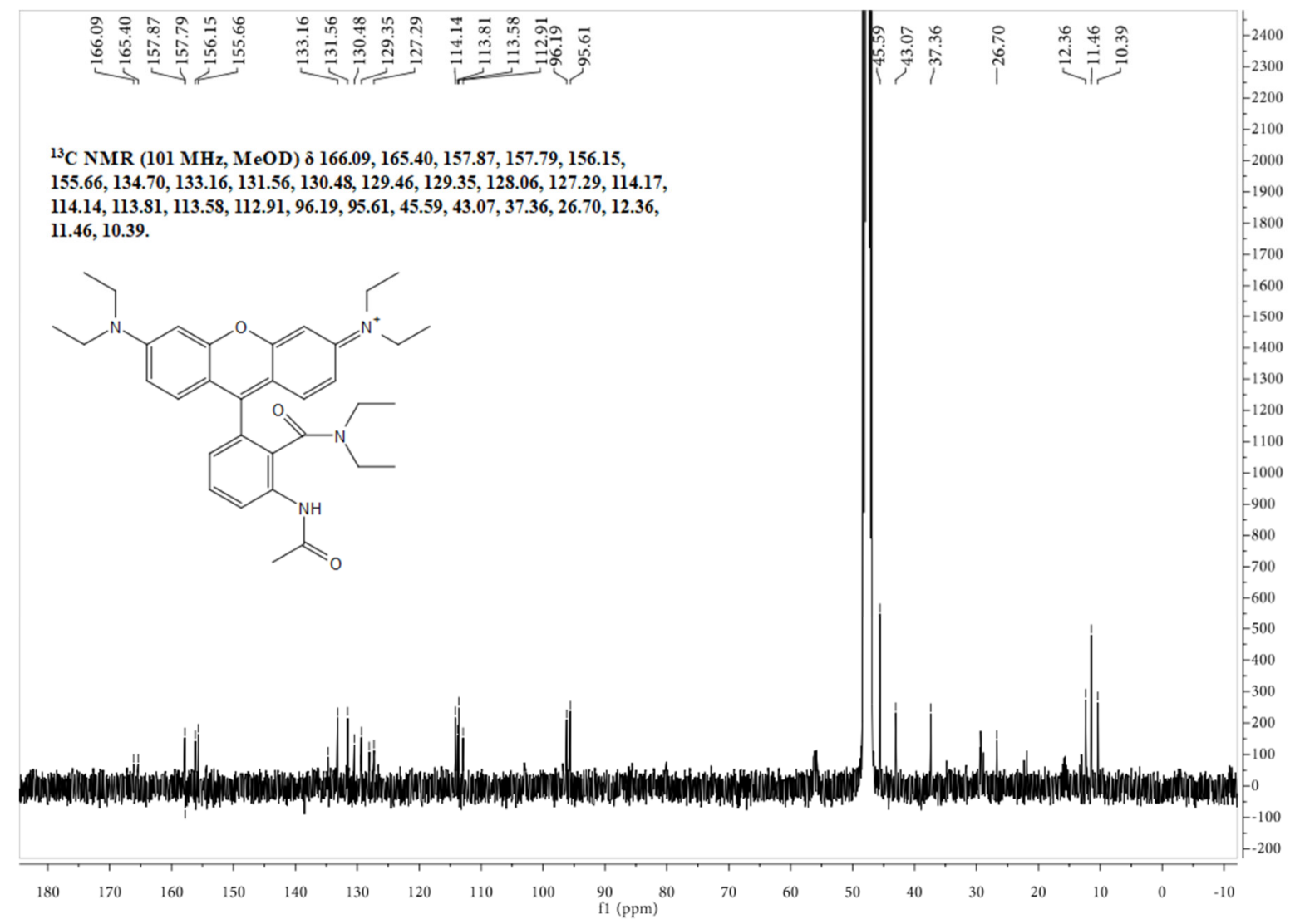

Figure S99. ${ }^{13} \mathrm{C}$ NMR spectrum of M2.

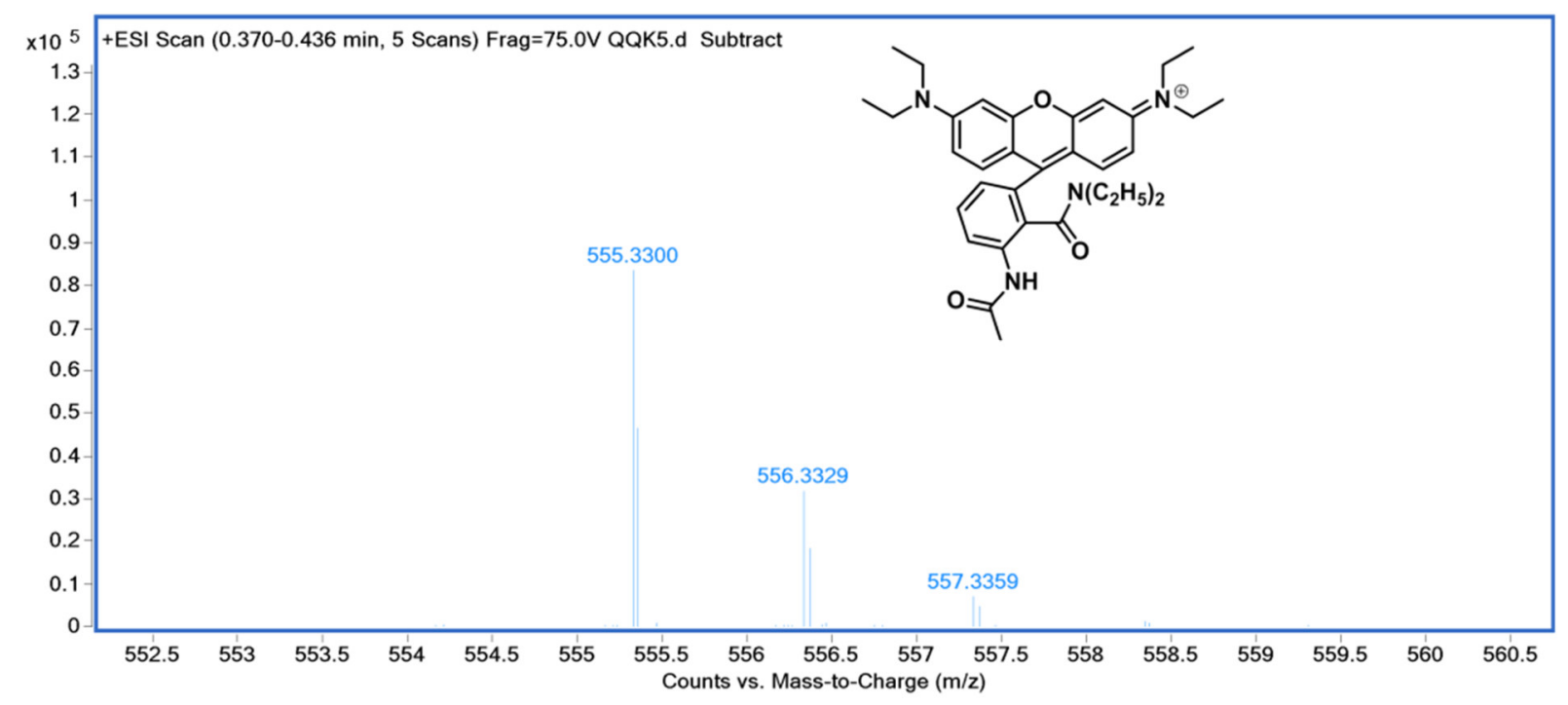

Figure S100. HRMS spectrum of M2. 


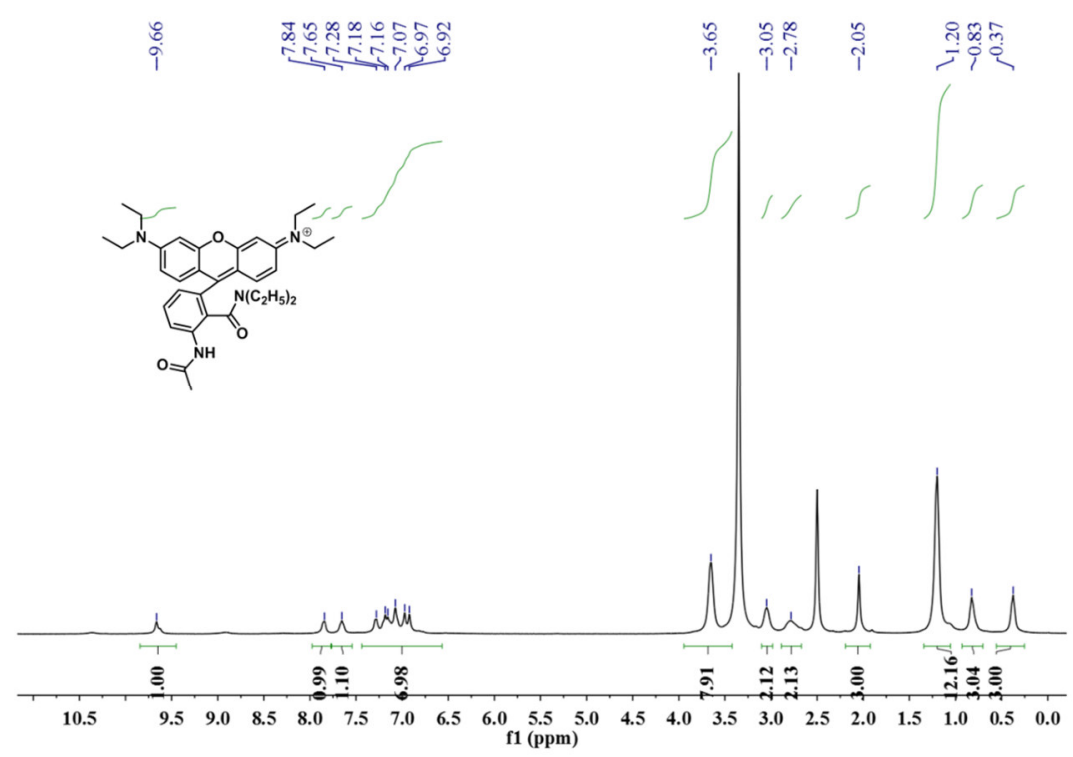

Figure S101. ${ }^{1} \mathrm{H}$ NMR spectrum of M3 in DMSO- $d_{6}$.

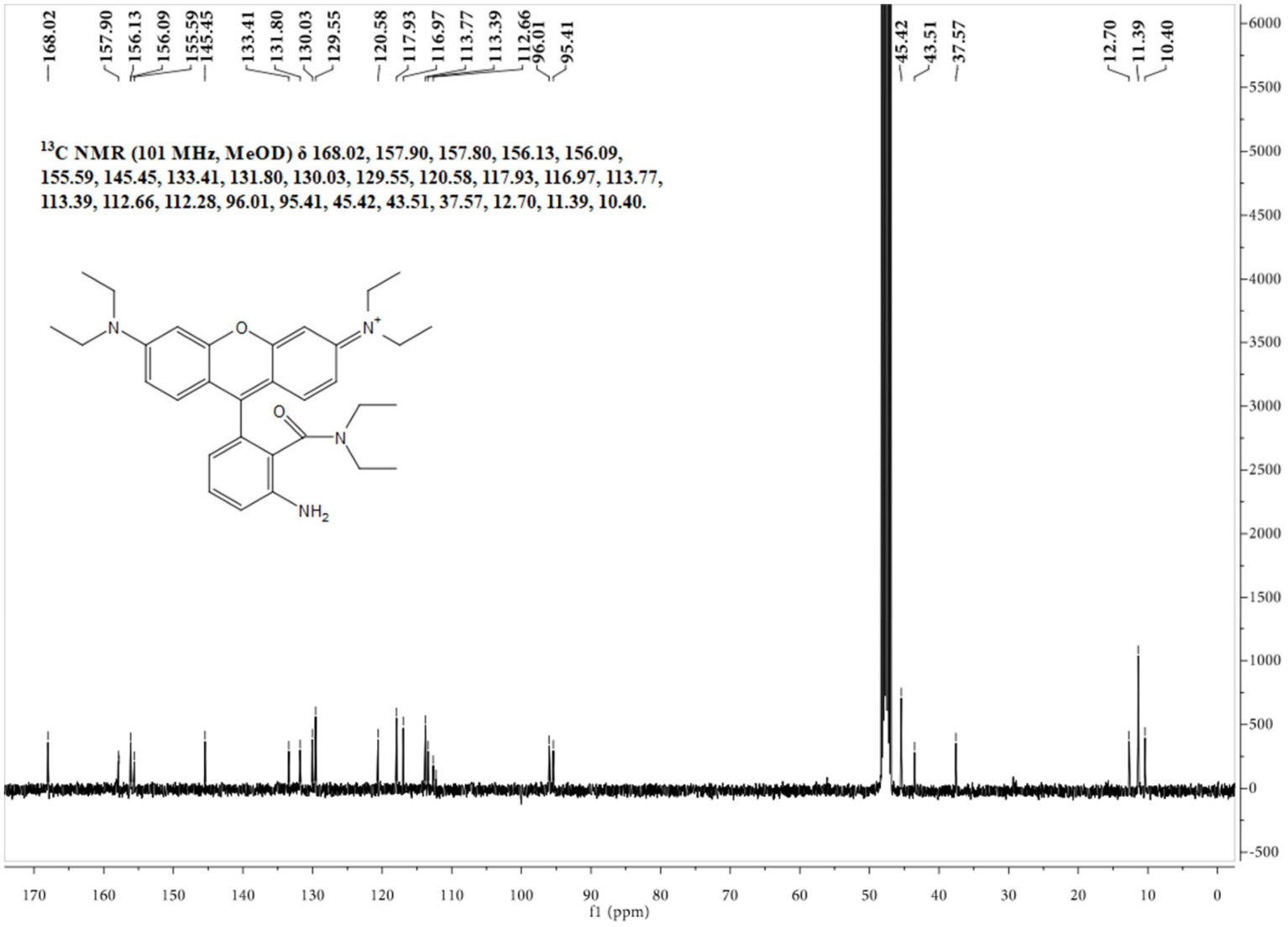

Figure $\mathrm{S} 102 .{ }^{13} \mathrm{C}$ NMR spectrum of $\mathbf{M} 3$. 


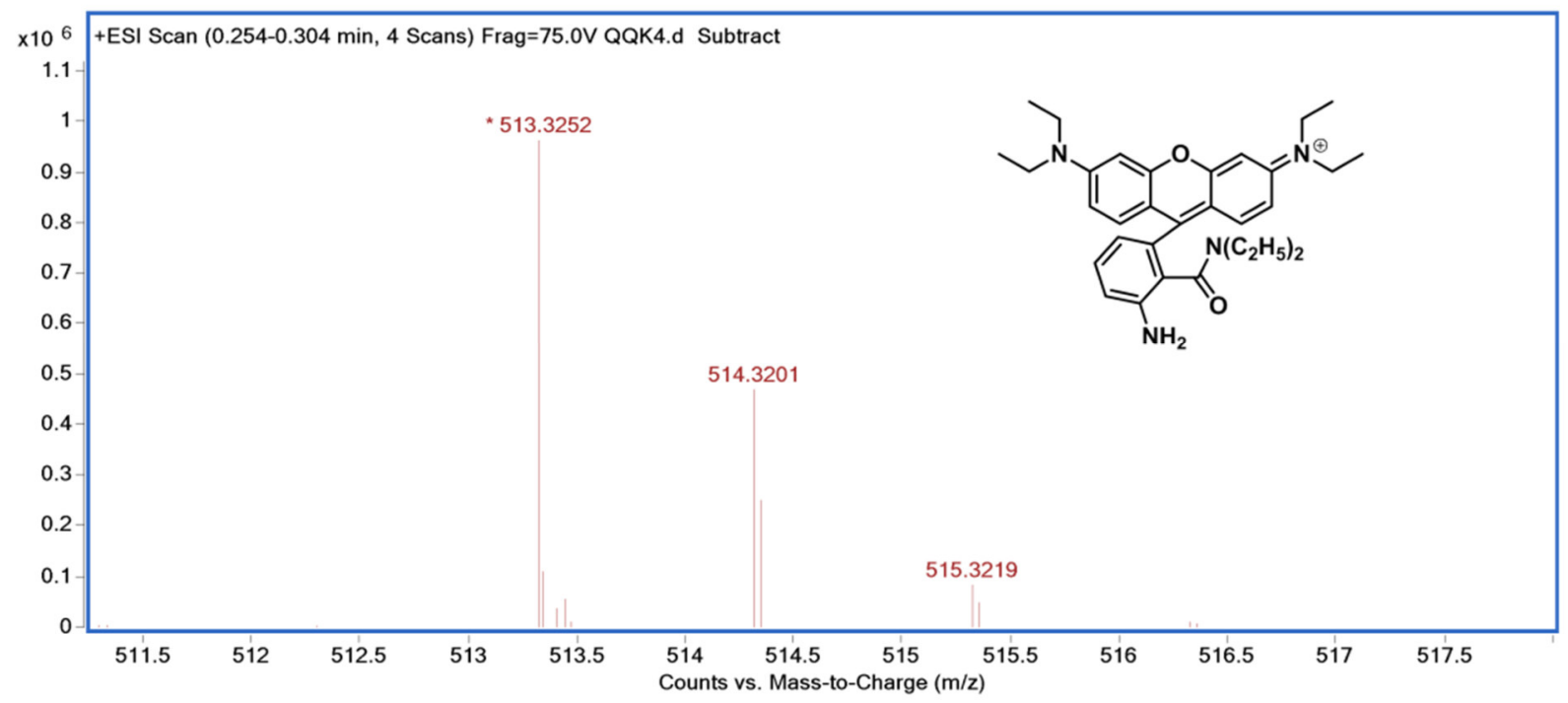

Figure S103. HRMS spectrum of M3.

UV-Vis absorption and fluorescence spectra of B1-B6

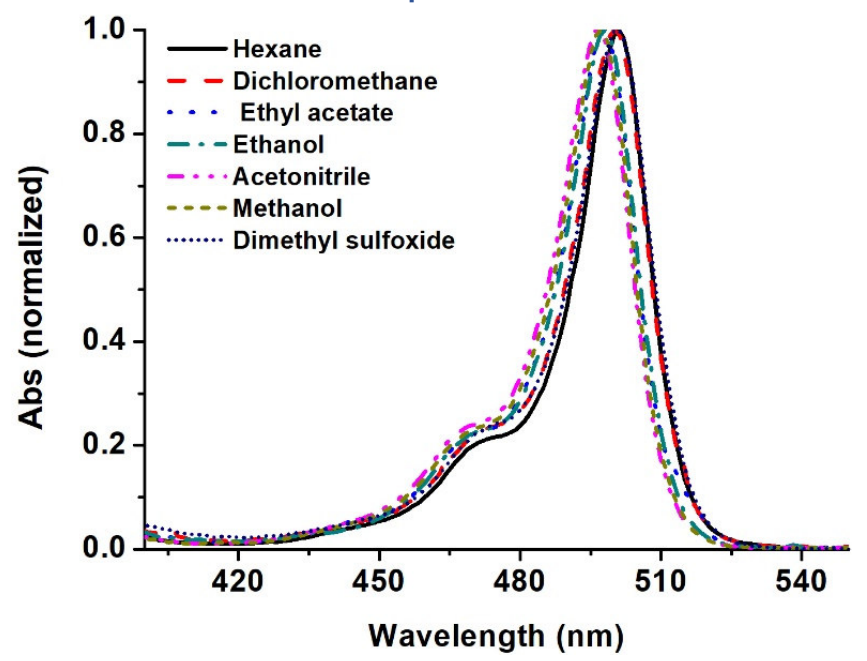

Figure S104. Normalized UV-Vis absorption spectra of B1 in hexane, dichloromethane, ethyl acetate, ethanol, acetonitrile, methanol, and dimethyl sulfoxide. [B1] $=2 \mu \mathrm{M}$. 


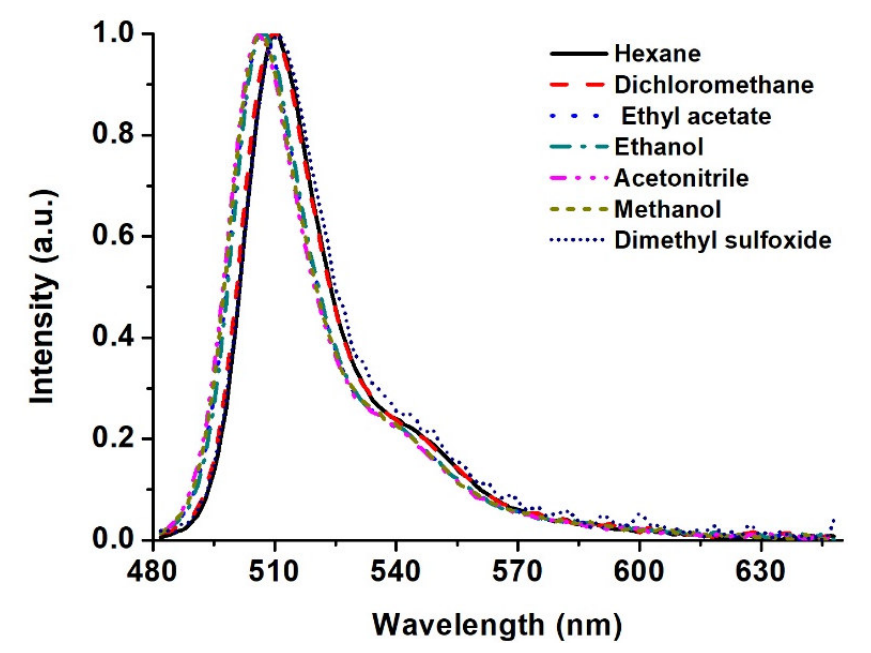

Figure S105. Normalized fluorescence spectra of B1 in hexane, dichloromethane, ethyl acetate, ethanol, acetonitrile, methanol, and dimethyl sulfoxide. [B1] $=2 \mu \mathrm{M}$; excitation wavelength $=470 \mathrm{~nm}$.

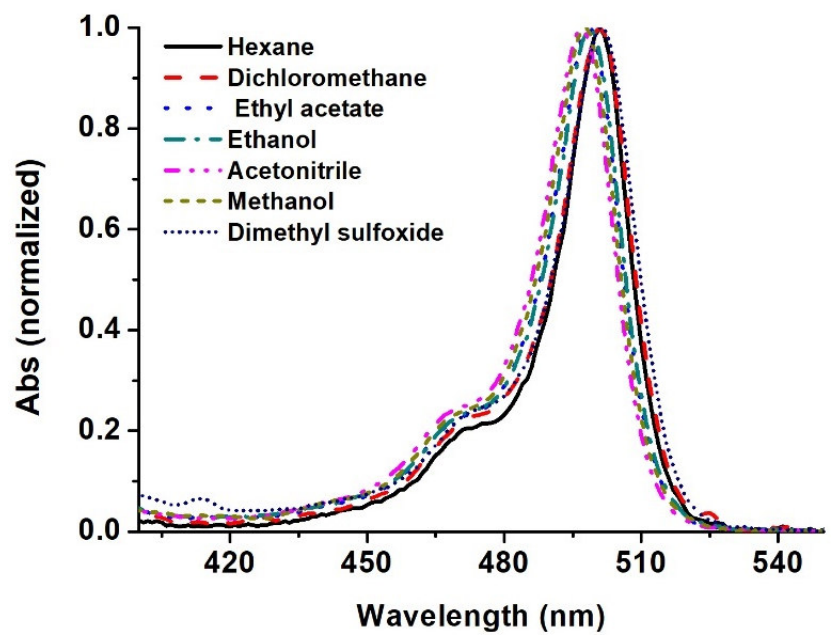

Figure S106. Normalized UV-Vis absorption spectra of B2 in hexane, dichloromethane, ethyl acetate, ethanol, acetonitrile, methanol, and dimethyl sulfoxide. [B2] $=2 \mu \mathrm{M}$. 


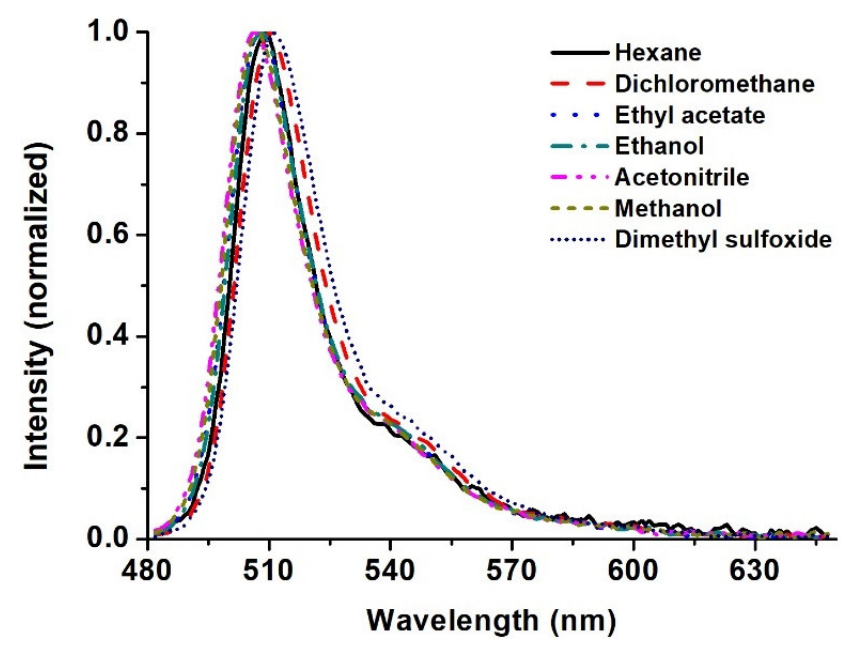

Figure S107. Normalized fluorescence spectra of B2 in hexane, dichloromethane, ethyl acetate, ethanol, acetonitrile, methanol, and dimethyl sulfoxide. [B2] = $2 \mu \mathrm{M}$; excitation wavelength $=470 \mathrm{~nm}$.

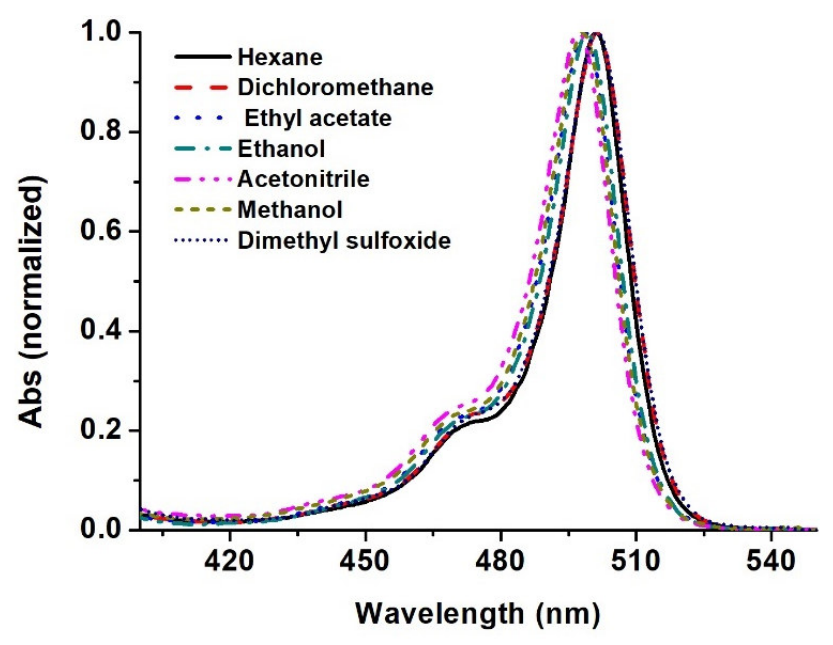

Figure S108. Normalized UV-Vis absorption spectra of B3 in hexane, dichloromethane, ethyl acetate, ethanol, acetonitrile, methanol, and dimethyl sulfoxide. [B3] $=2 \mu \mathrm{M}$. 


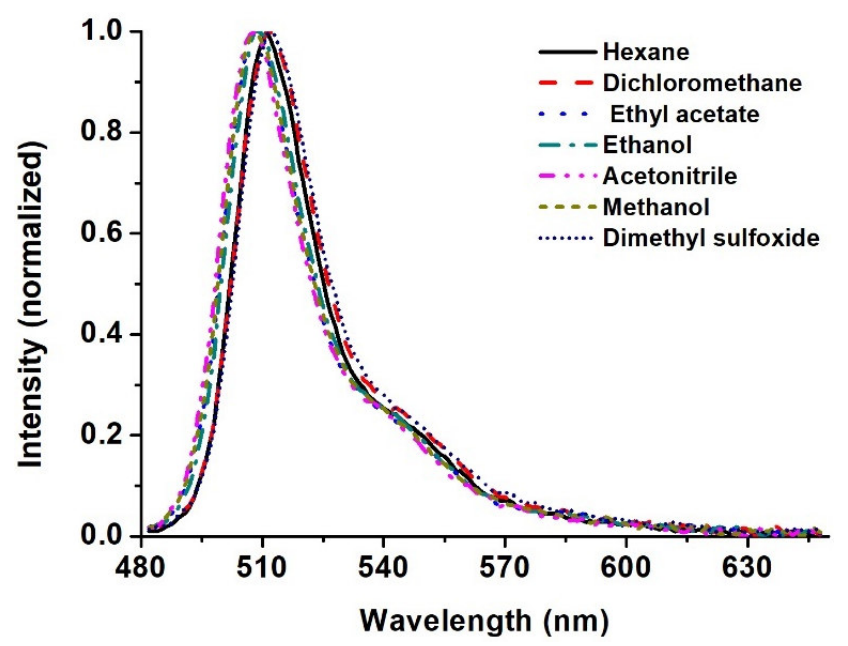

Figure S109. Normalized fluorescence spectra of B3 in hexane, dichloromethane, ethyl acetate, ethanol, acetonitrile, methanol, and dimethyl sulfoxide. [B3] $=2 \mu \mathrm{M}$; excitation wavelength $=470 \mathrm{~nm}$.

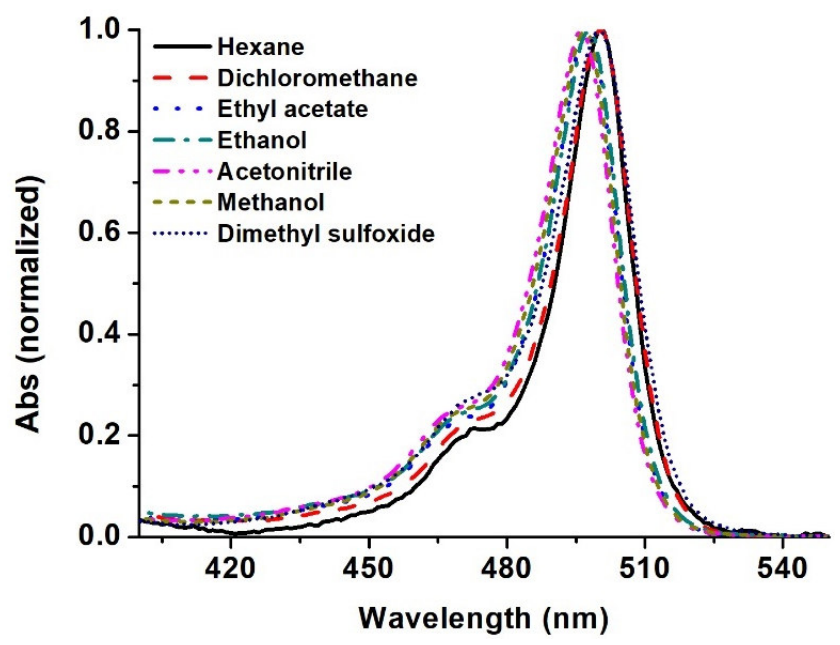

Figure S110. Normalized UV-Vis absorption spectra of B4 in hexane, dichloromethane, ethyl acetate, ethanol, acetonitrile, methanol, and dimethyl sulfoxide. [B4] $=2 \mu \mathrm{M}$. 


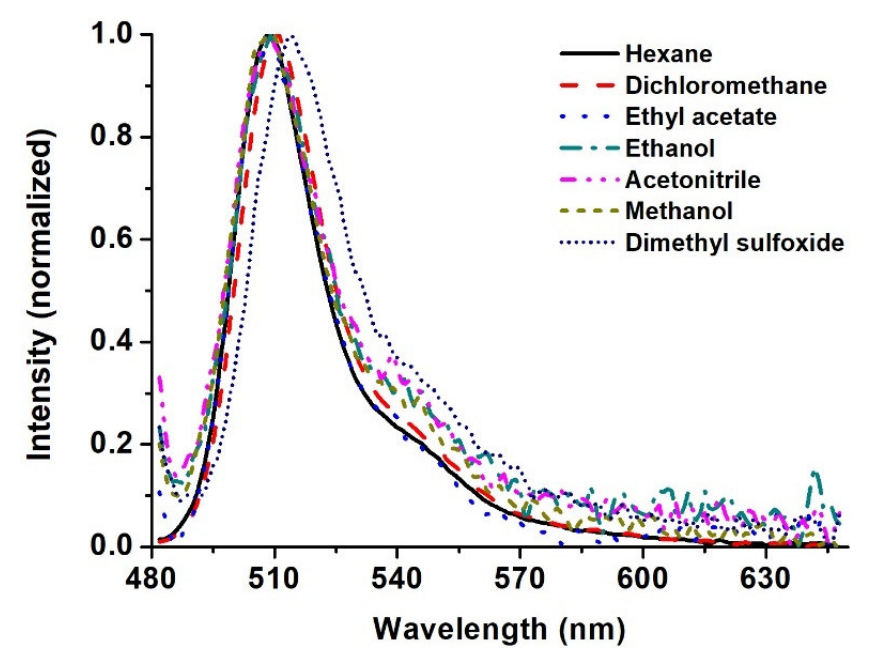

Figure S111. Normalized fluorescence spectra of B4 in hexane, dichloromethane, ethyl acetate, ethanol, acetonitrile, methanol, and dimethyl sulfoxide. [B4] = $2 \mu \mathrm{M}$; excitation wavelength $=470 \mathrm{~nm}$.

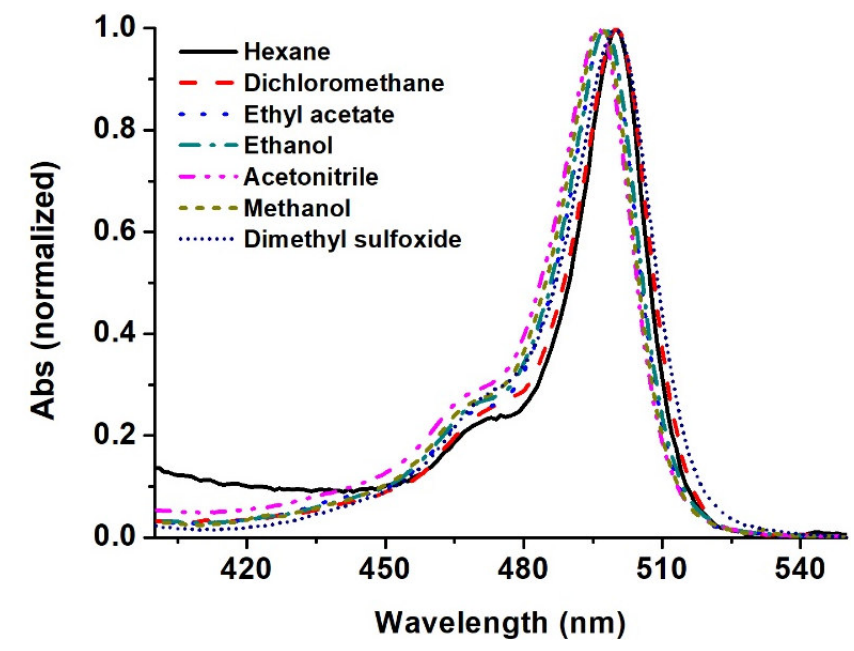

Figure S112. Normalized UV-Vis absorption spectra of B5 in hexane, dichloromethane, ethyl acetate, ethanol, acetonitrile, methanol, and dimethyl sulfoxide. [B5] = $2 \mu \mathrm{M}$. 


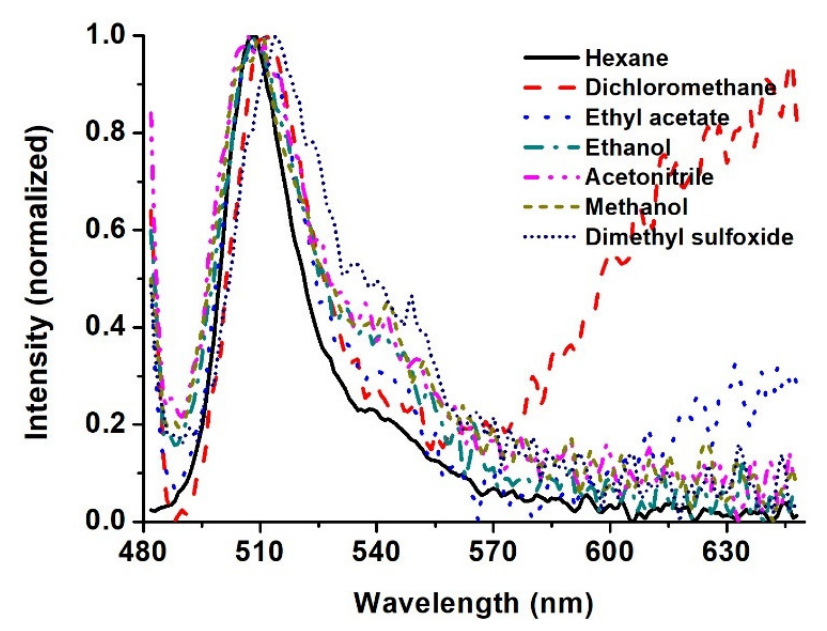

Figure S113. Normalized fluorescence spectra of B5 in hexane, dichloromethane, ethyl acetate, ethanol, acetonitrile, methanol, and dimethyl sulfoxide. [B5] = $2 \mu \mathrm{M}$; excitation wavelength $=470 \mathrm{~nm}$.

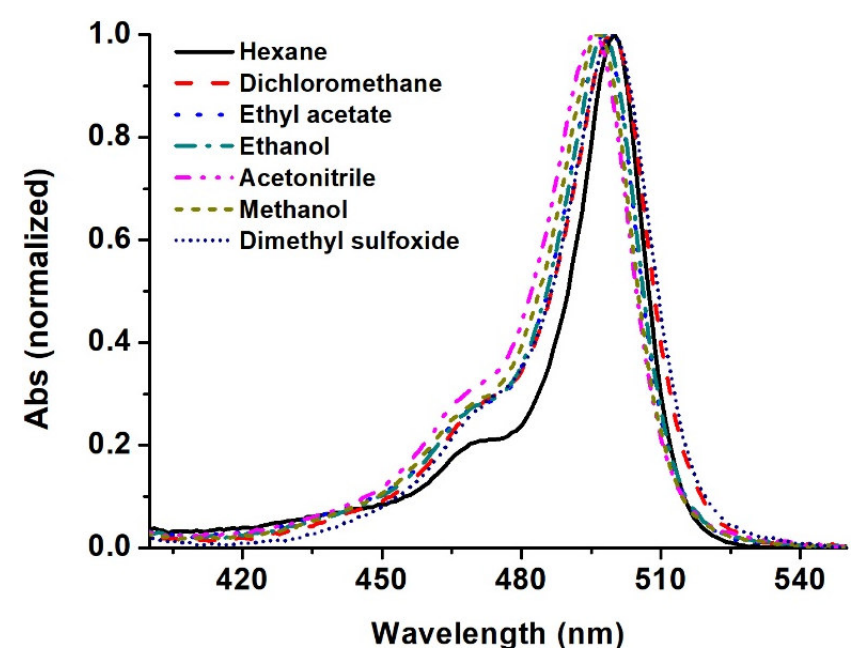

Figure S114. Normalized UV-Vis absorption spectra of B6 in hexane, dichloromethane, ethyl acetate, ethanol, acetonitrile, methanol, and dimethyl sulfoxide. [B6] $=2 \mu \mathrm{M}$. 


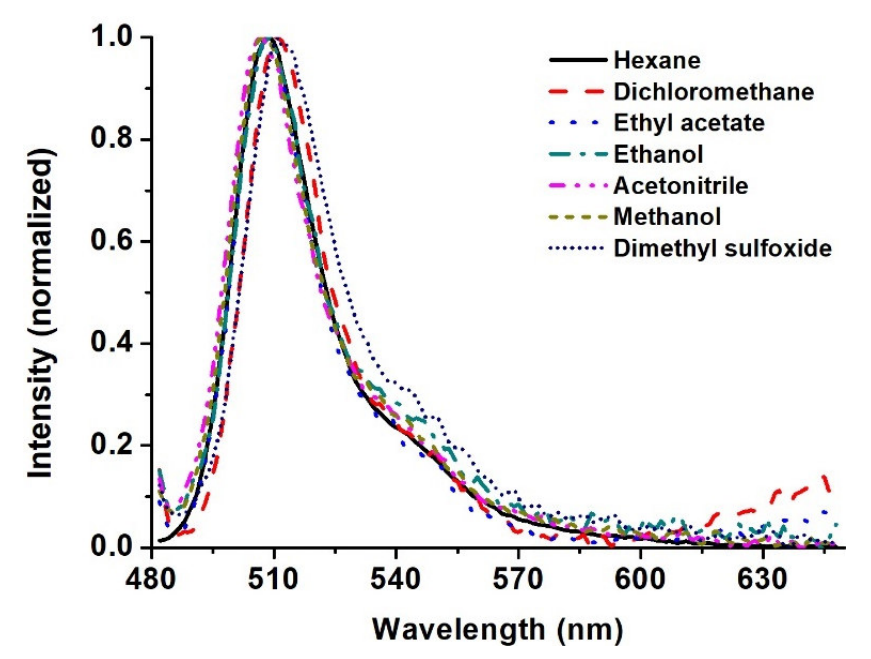

Figure S115. Normalized fluorescence spectra of B6 in hexane, dichloromethane, ethyl acetate, ethanol, acetonitrile, methanol, and dimethyl sulfoxide. [B6] $=2 \mu \mathrm{M}$; excitation wavelength $=470 \mathrm{~nm}$.

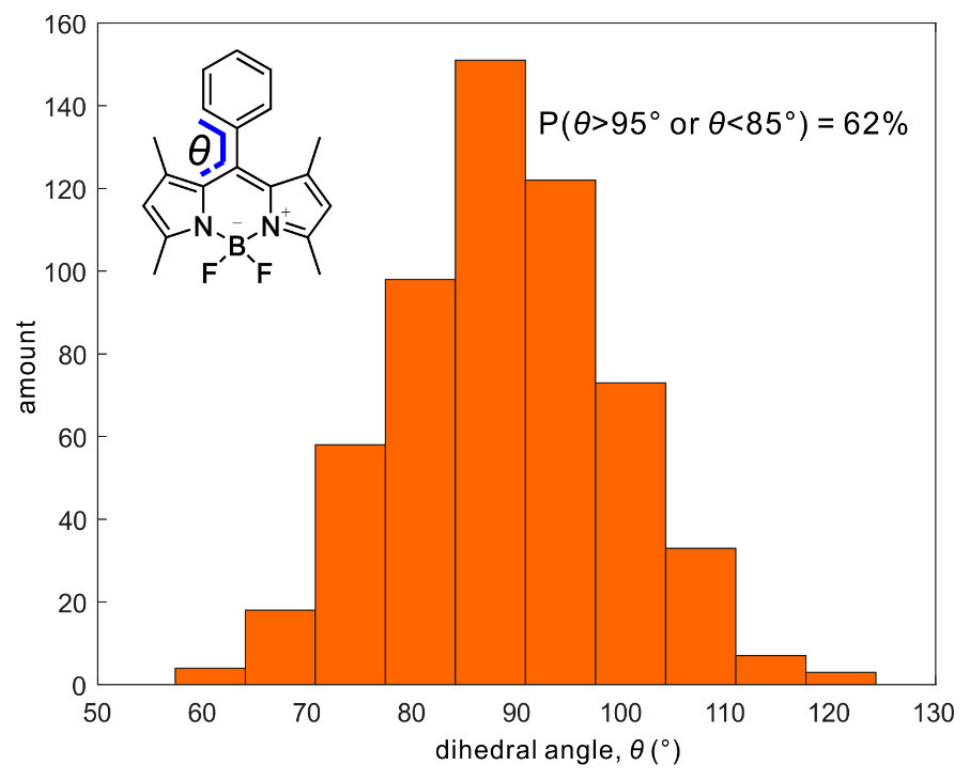

Figure S116. Histogram of the dihedral angle, $\theta$, in tetramethyl BODIPY dyes, as searched from the Cambridge Crystallographic Data Centre (CCDC); the inset shows the search moiety used in the data-mining, and the probability, $\mathrm{P}$, of $\theta$ being greater than $95^{\circ}$ or smaller than $85^{\circ}$. 

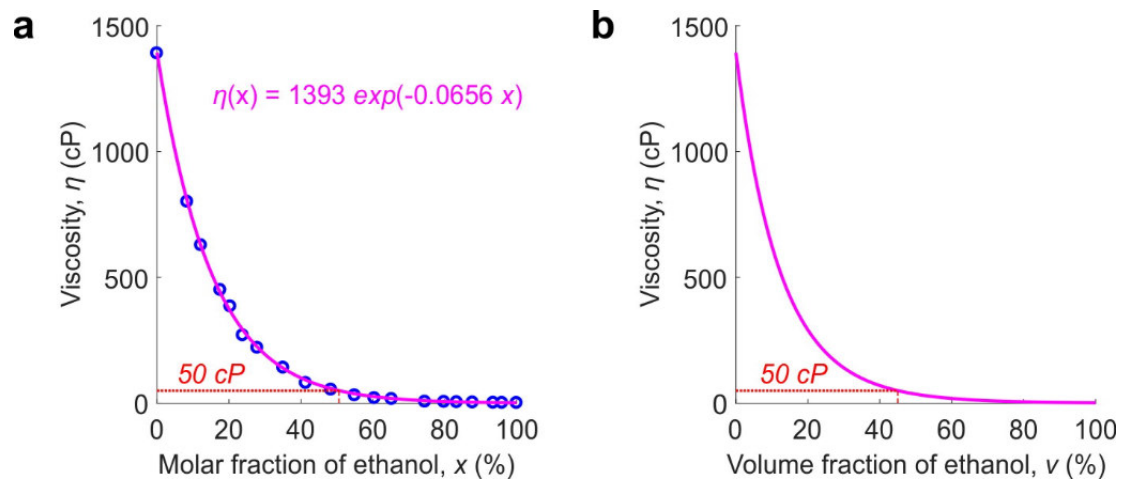

Figure S117. Calibration curve of the viscosity $(\eta)$ of ethanol-glycerol mixture at $21^{\circ} \mathrm{C}$, as a function of (a) the molar fraction of ethanol, $x(\%)$; and (b) the volume fraction of ethanol, $v(\%) .50 \mathrm{cP}$ corresponds to $\sim 50 \%$ of molar fraction and $\sim 45 \%$ of volume fraction of ethanol. The raw data for constructing these calibration curves were extracted from Reference 36.

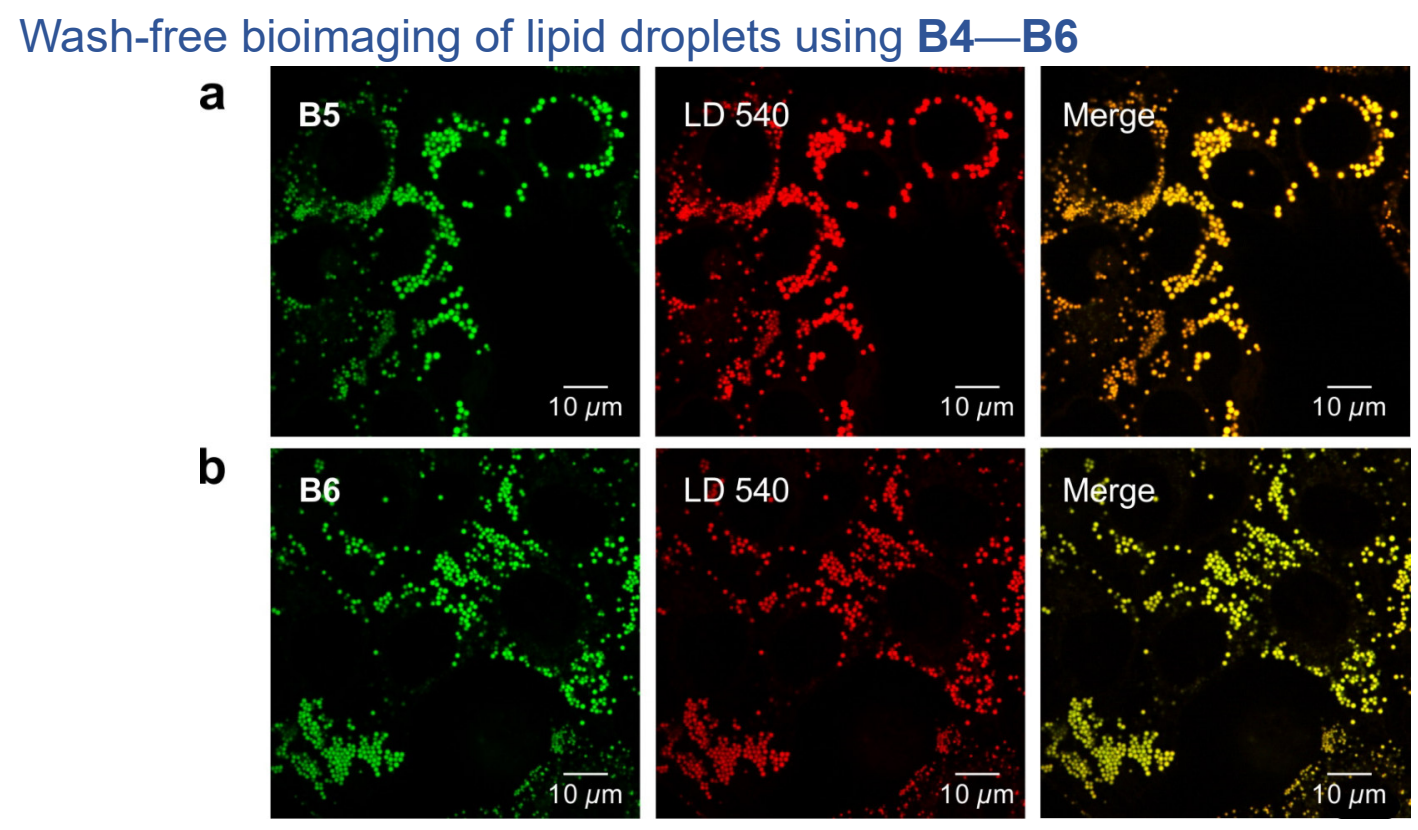

Figure S118. a) Co-staining of HeLa cells using B5 (1 $\mu \mathrm{M})$ and LD $540(1 \mu \mathrm{M})$; green channel, B5; red channel, LD 540; yellow channel, the merged image. b) Co-staining of HeLa cells using B6 (1 $\mu$ M) and LD 540 (1 1 M); green channel, B6; red channel, LD 540; yellow channel, the merged image. 

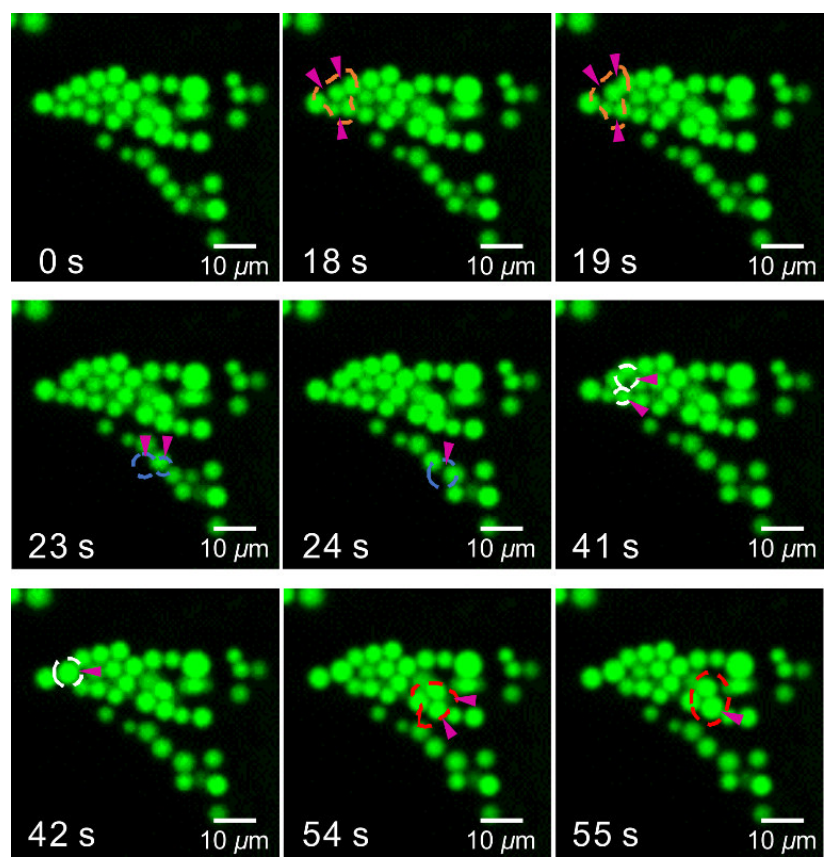

Figure S119. Lipid droplet dynamics of HeLa cells, as revealed by B4.

Viscosity dependence of the UV-Vis absorption and emission spectra of B4B6
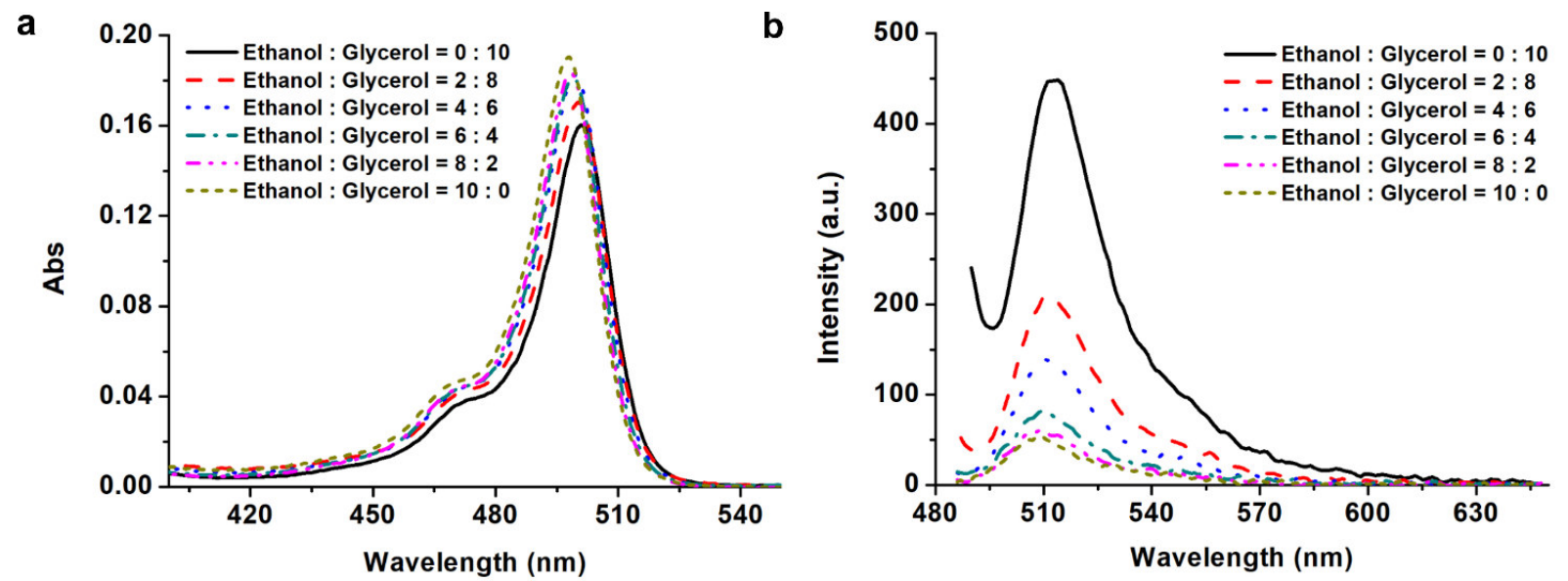

Figure S120. UV-Vis absorption spectra (a) and fluorescence intensity changes (b) of B4 as a function of viscosity in the mixture of ethanol and glycerol. [B4] $=2 \mu \mathrm{M}$; excitation wavelength $=470 \mathrm{~nm}$. 
a

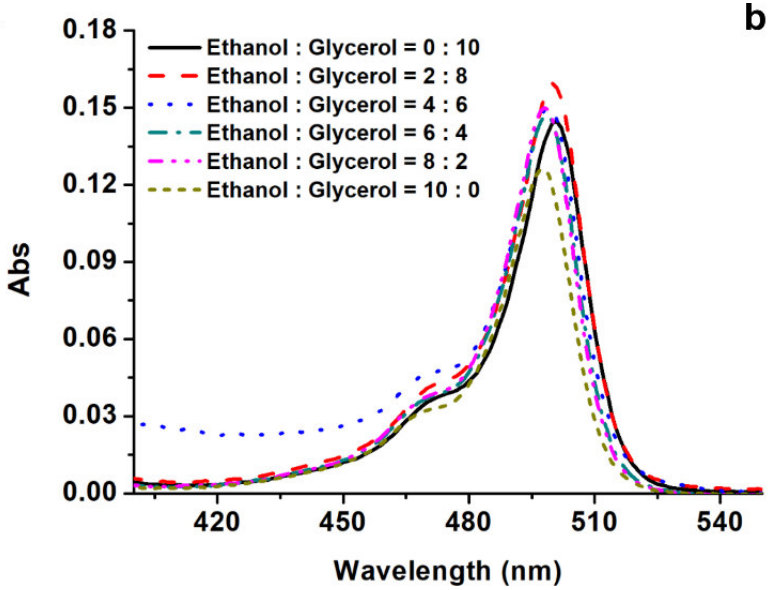

b

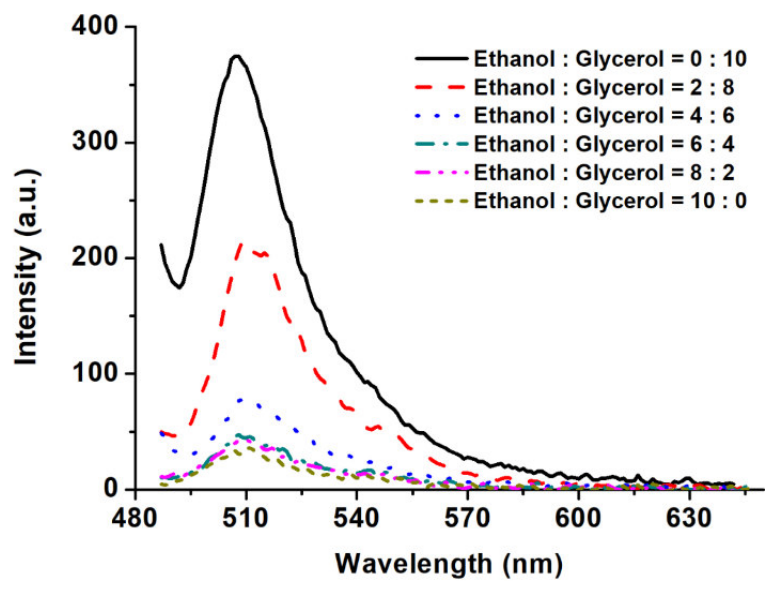

Figure S121. UV-Vis absorption spectra (a) and fluorescence intensity changes (b) of B5 as a function of viscosity in the mixture of ethanol and glycerol. $[\mathrm{B} 5]=2 \mu \mathrm{M}$; excitation wavelength $=470 \mathrm{~nm}$.
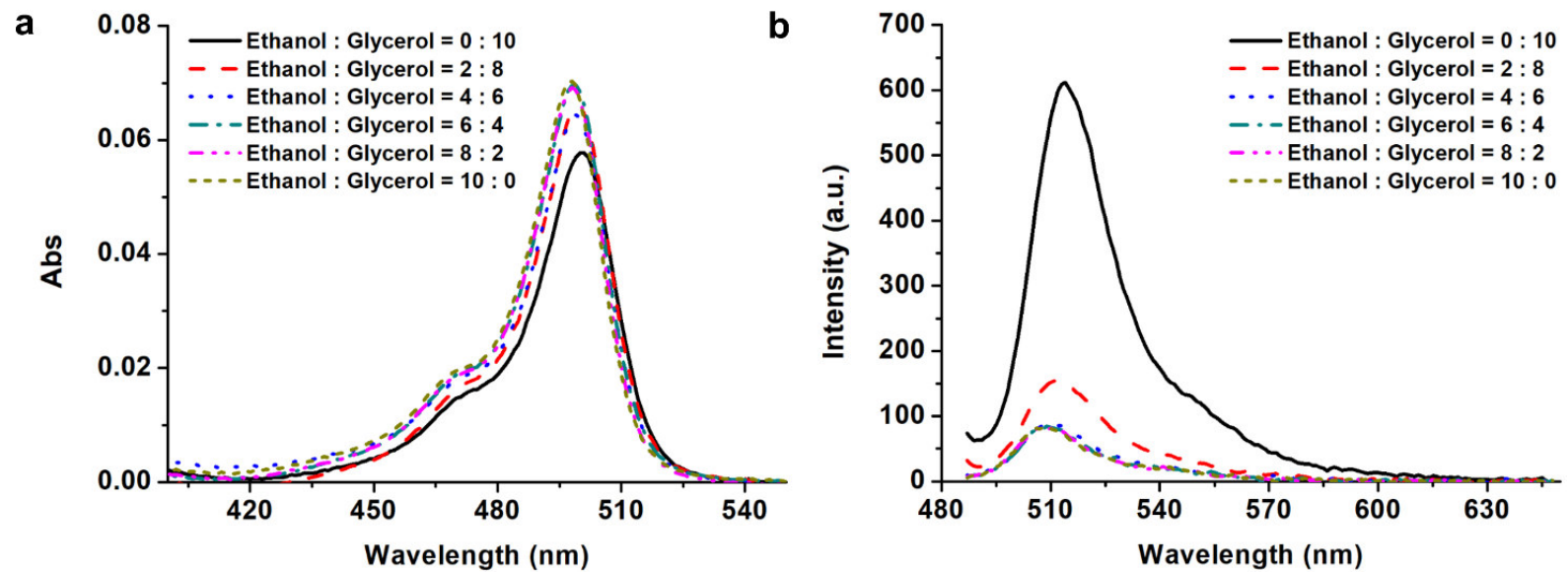

Figure S122. UV-Vis absorption spectra (a) and fluorescence intensity changes (b) of B6 as a function of viscosity in the mixture of ethanol and glycerol. $[\mathrm{B} 6]=2 \mu \mathrm{M}$; excitation wavelength $=470 \mathrm{~nm}$. 
Calculated frontier molecular orbitals and corresponding $\Delta \mathrm{E}$ of meso-phenyl substituted fluorescein and rhodamine derivatives
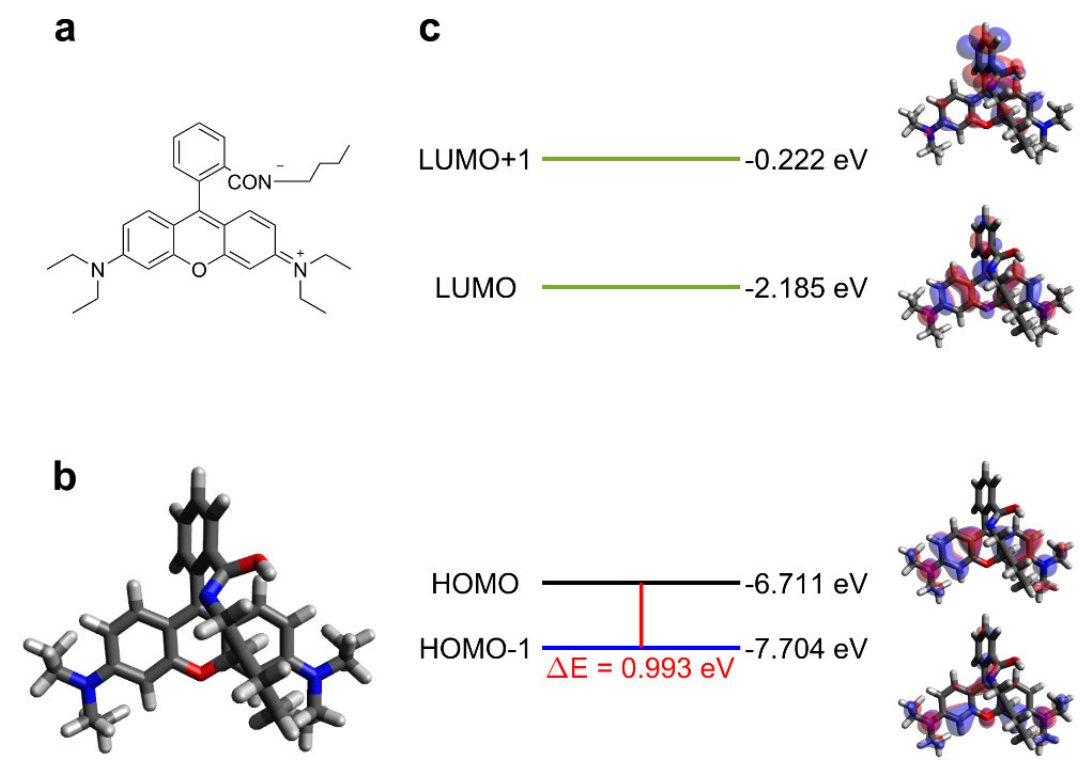

Figure S123. a) Molecular structure, b) optimized structure, c) frontier molecular orbitals and corresponding energy levels, and calculated $\Delta \mathrm{E}$ of R1 in the ground state in water at M062X/Def2SVP level.
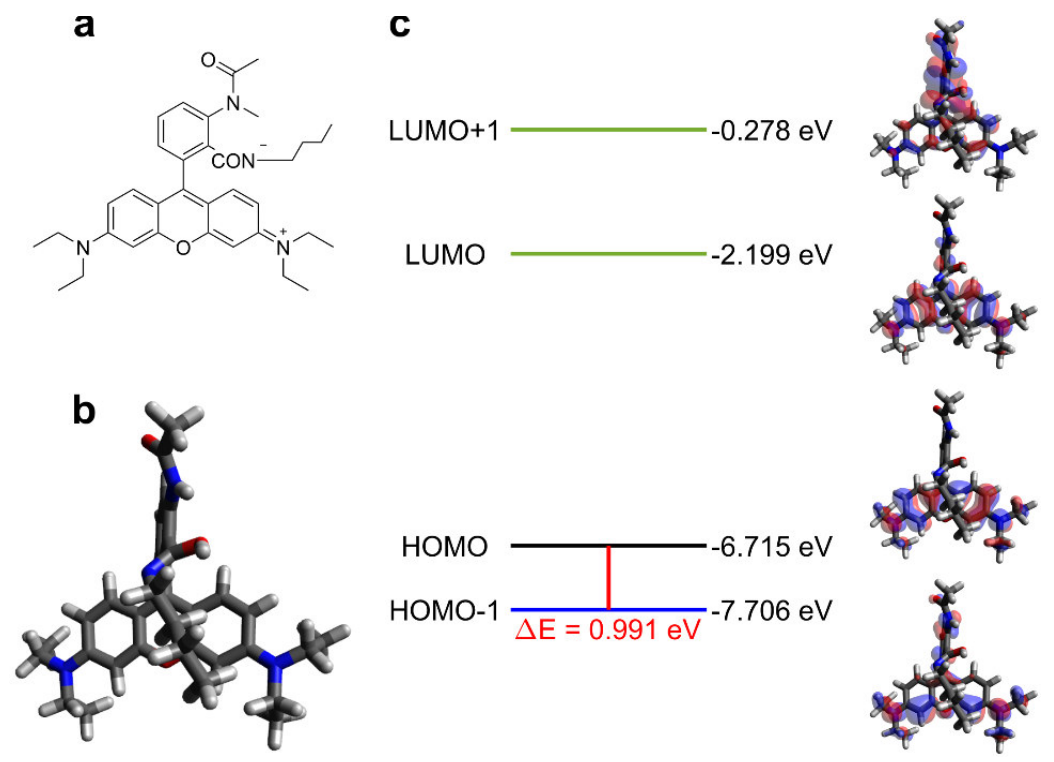

Figure S124. a) Molecular structure, b) optimized structure, c) frontier molecular orbitals and corresponding energy levels, and calculated $\Delta \mathrm{E}$ of $\mathbf{R} 2$ in the ground state in water at M062X/Def2SVP level. 
a<smiles></smiles>

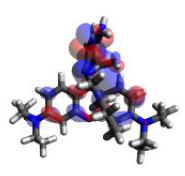

LUMO $-2.161 \mathrm{eV}$

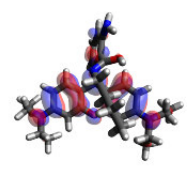

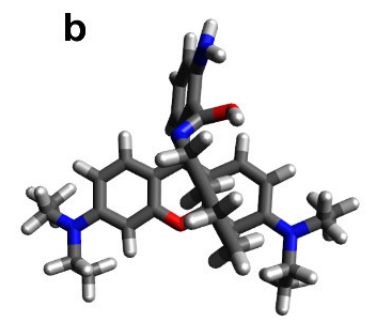
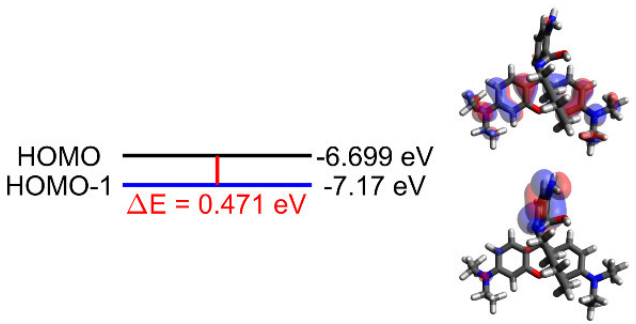

Figure S125. a) Molecular structure, b) optimized structure, c) frontier molecular orbitals and corresponding energy levels, and calculated $\Delta \mathrm{E}$ of $\mathbf{R 3}$ in the ground state in water at M062X/Def2SVP level.

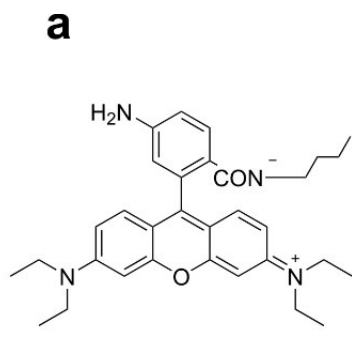

\section{C}

$\mathrm{LUMO}+1 \longrightarrow 0.002 \mathrm{eV}$

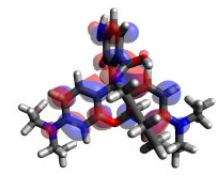

LUMO $-2.155 \mathrm{eV}$
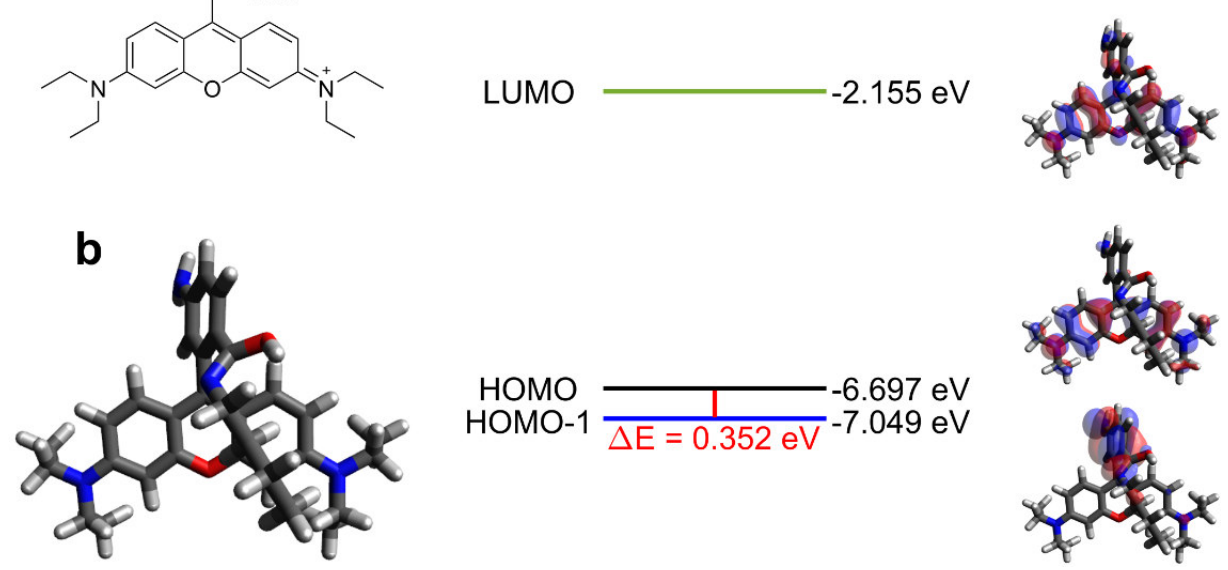

Figure S126. a) Molecular structure, b) optimized structure, c) frontier molecular orbitals and corresponding energy levels, and calculated $\Delta \mathrm{E}$ of R4 in the ground state in water at M062X/Def2SVP level. 

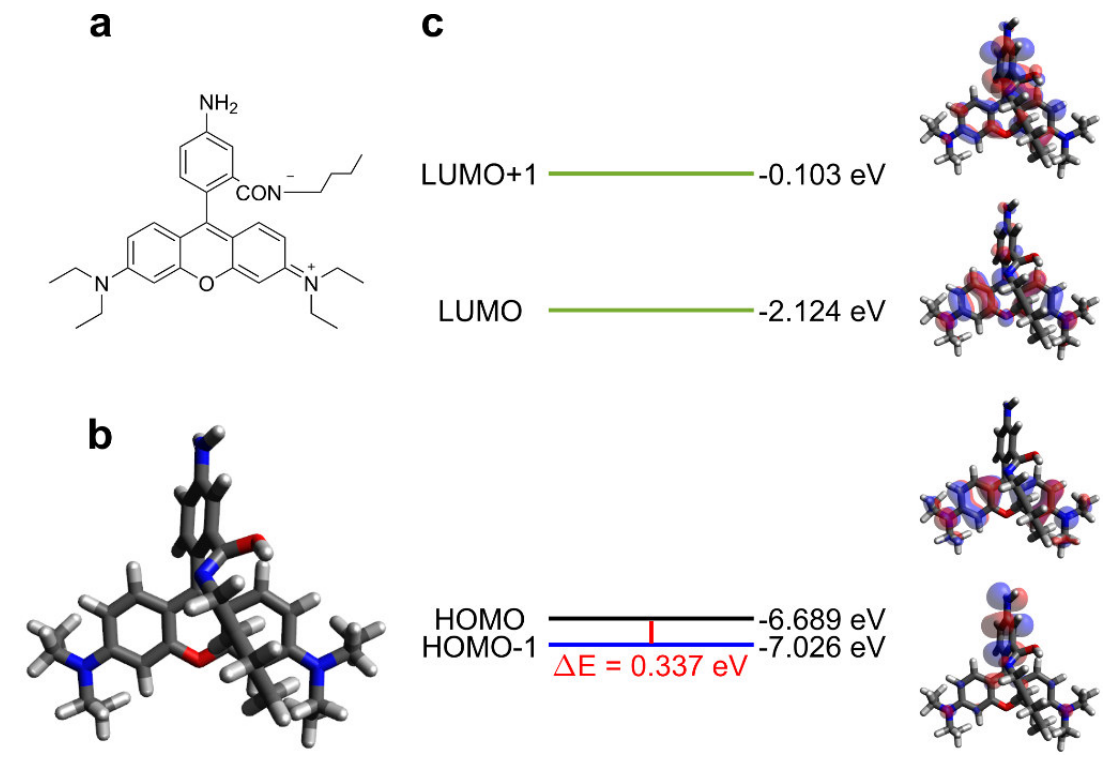

Figure S127. a) Molecular structure, b) optimized structure, c) frontier molecular orbitals and corresponding energy levels, and calculated $\Delta \mathrm{E}$ of $\mathbf{R 5}$ in the ground state in water at M062X/Def2SVP level.
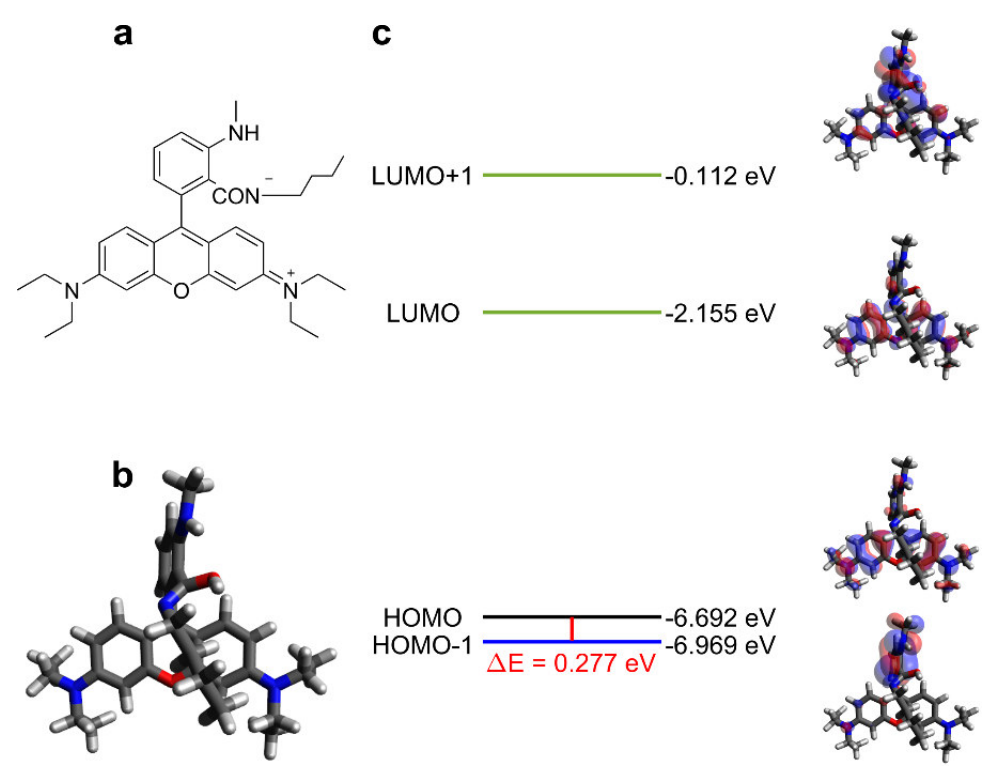

Figure S128. a) Molecular structure, b) optimized structure, c) frontier molecular orbitals and corresponding energy levels, and calculated $\Delta \mathrm{E}$ of $\mathbf{R} \mathbf{6}$ in the ground state in water at M062X/Def2SVP level. 

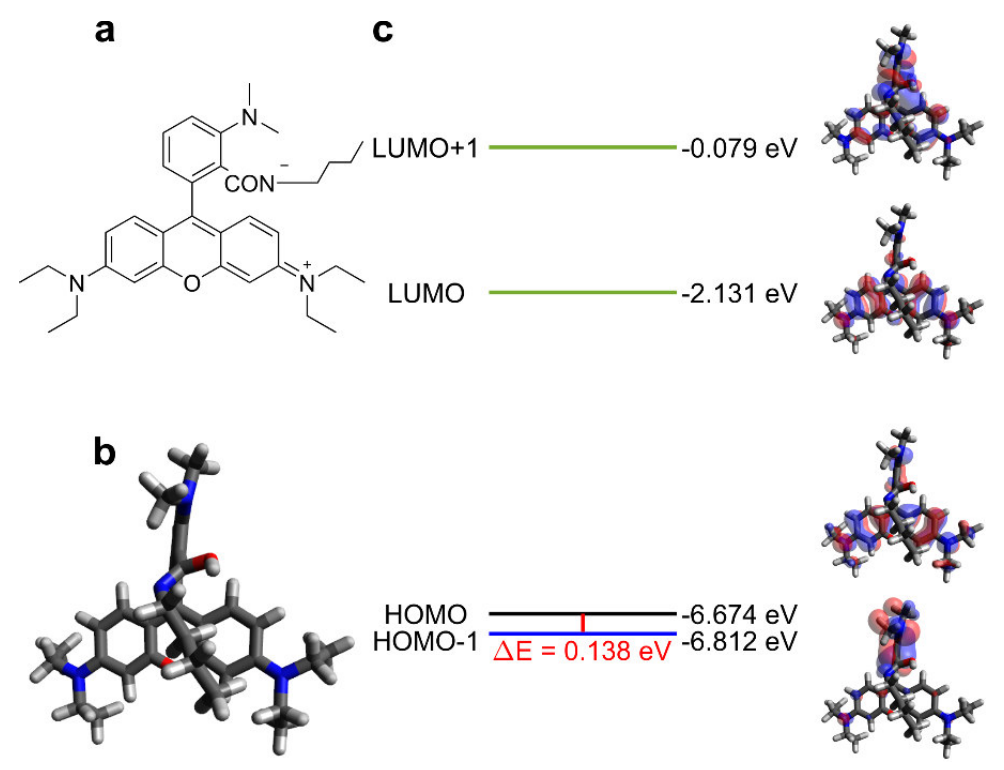

Figure S129. a) Molecular structure, b) optimized structure, c) frontier molecular orbitals and corresponding energy levels, and calculated $\Delta \mathrm{E}$ of $\mathbf{R 7}$ in the ground state in water at M062X/Def2SVP level.

a<smiles>CN(C)c1ccc2c(c1)[Si](C)(C)C1=CC(=[N+](C)C)C=CC1=C2c1ccccc1</smiles>

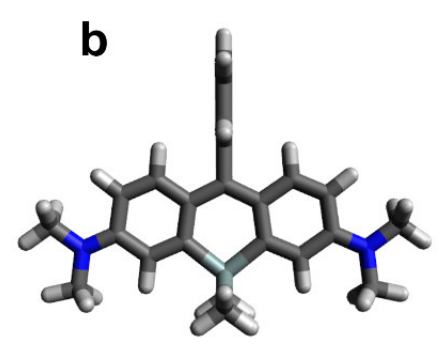

C<smiles>CCCCO[Mg]</smiles>

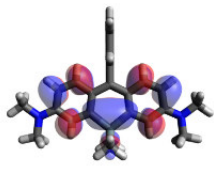

HOMO

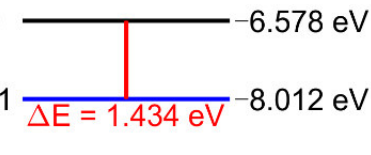

HOMO-1 $\frac{}{\Delta \mathrm{E}=1.434 \mathrm{eV}}-8.012 \mathrm{eV}$

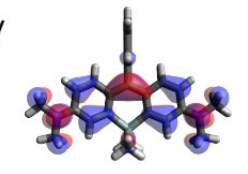

Figure S130. a) Molecular structure, b) optimized structure, c) frontier molecular orbitals and corresponding energy levels, and calculated $\Delta \mathrm{E}$ of $\mathbf{R 8}$ in the ground state in PBS at M062X/Def2SVP level. 
a<smiles>CN(C)c1ccc2c(c1)[Si](C)(C)C1=CC(=[N+](C)C)C=CC1=C2c1ccccc1F</smiles>

C

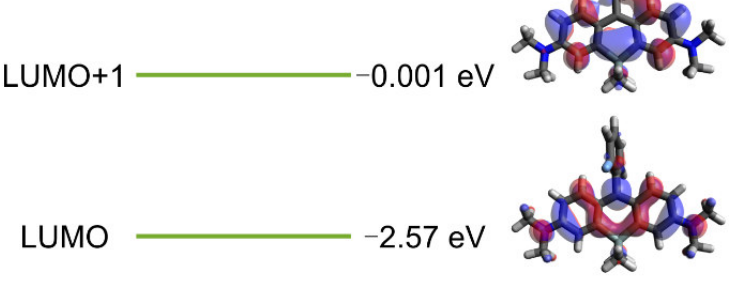

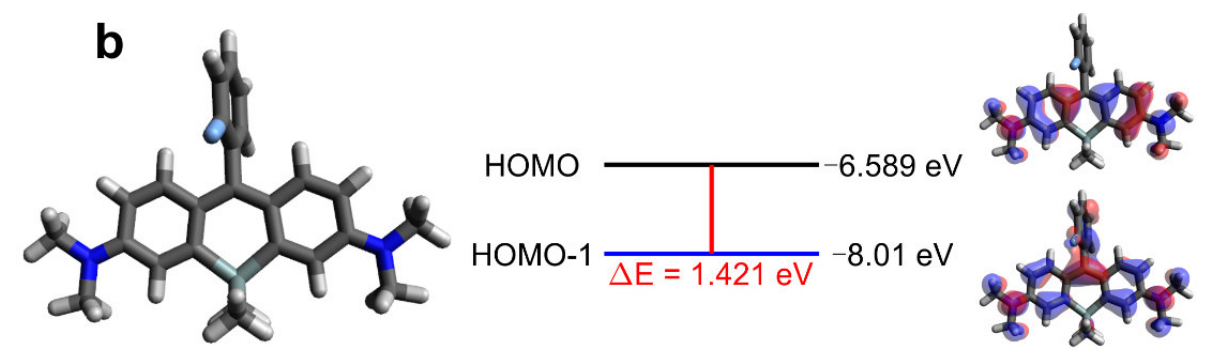

Figure S131. a) Molecular structure, b) optimized structure, c) frontier molecular orbitals and corresponding energy levels, and calculated $\Delta \mathrm{E}$ of R9 in the ground state in PBS at M062X/Def2SVP level.

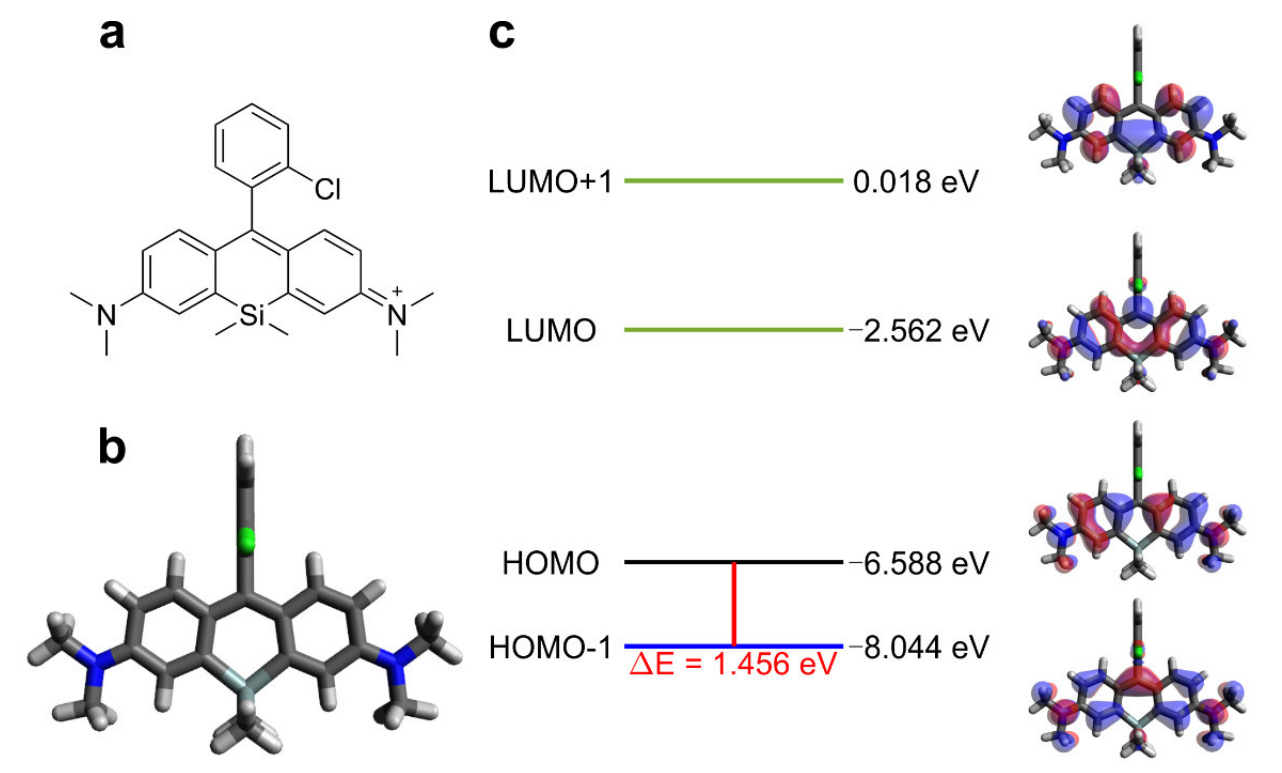

Figure S132. a) Molecular structure, b) optimized structure, c) frontier molecular orbitals and corresponding energy levels, and calculated $\Delta \mathrm{E}$ of R10 in the ground state in PBS at M062X/Def2SVP level. 
a<smiles>Cc1ccccc1C1=C2C=CC(=[N+](C)C)C=C2[Si](C)(C)c2cc(N(C)C)ccc21</smiles>

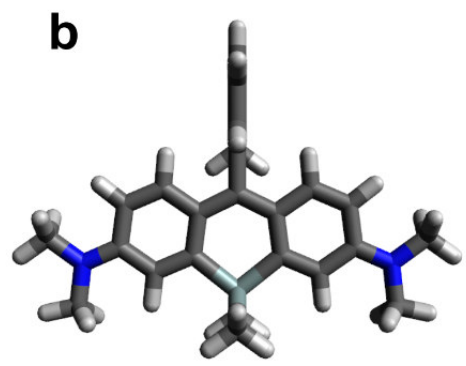

C

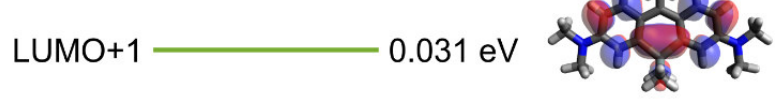

$-2.51 \mathrm{eV}$
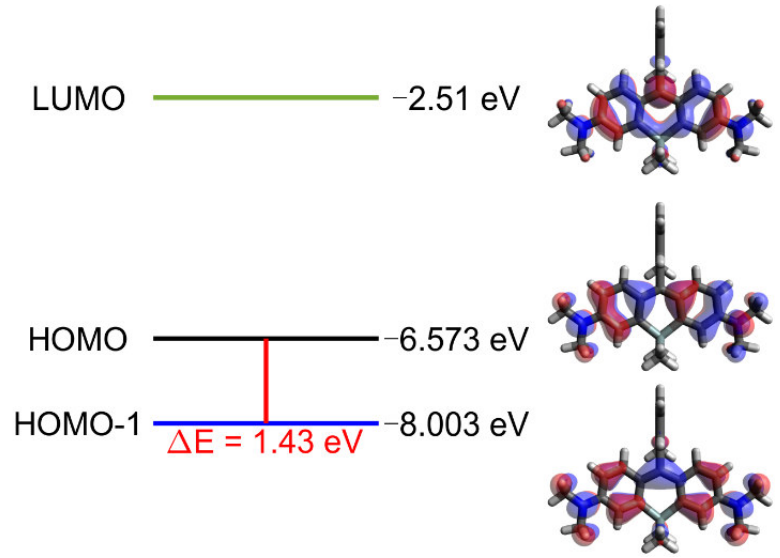

Figure S133. a) Molecular structure, b) optimized structure, c) frontier molecular orbitals and corresponding energy levels, and calculated $\Delta \mathrm{E}$ of R11 in the ground state in PBS at M062X/Def2SVP level.

a<smiles>CN(C)c1ccc2c(c1)[Si](C)(C)C1=CC(=[N+](C)C)C=CC1=C2c1ccccc1C(F)(F)F</smiles>

b

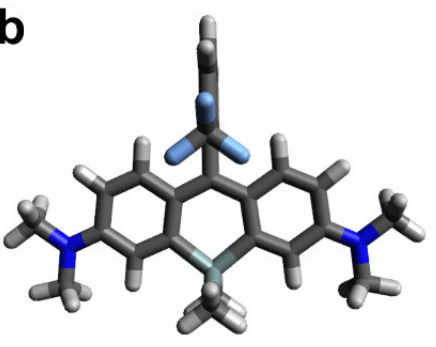

C<smiles>[Mg]O[Mg]</smiles>
$0.149 \mathrm{eV}$

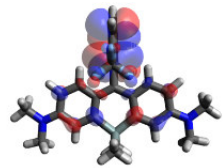
$-2.586 \mathrm{eV}$

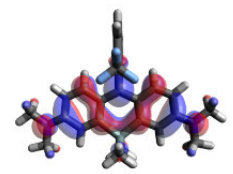

$\mathrm{HOMO}$ $-6.577 \mathrm{eV}$

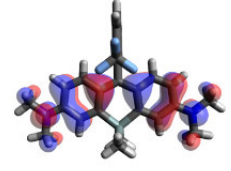

HOMO-1 $\frac{}{\Delta E=1.484 \mathrm{eV}^{-8.061} \mathrm{eV}}$

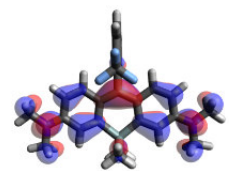

Figure S134. a) Molecular structure, b) optimized structure, c) frontier molecular orbitals and corresponding energy levels, and calculated $\Delta \mathrm{E}$ of R12 in the ground state in PBS at M062X/Def2SVP level. 

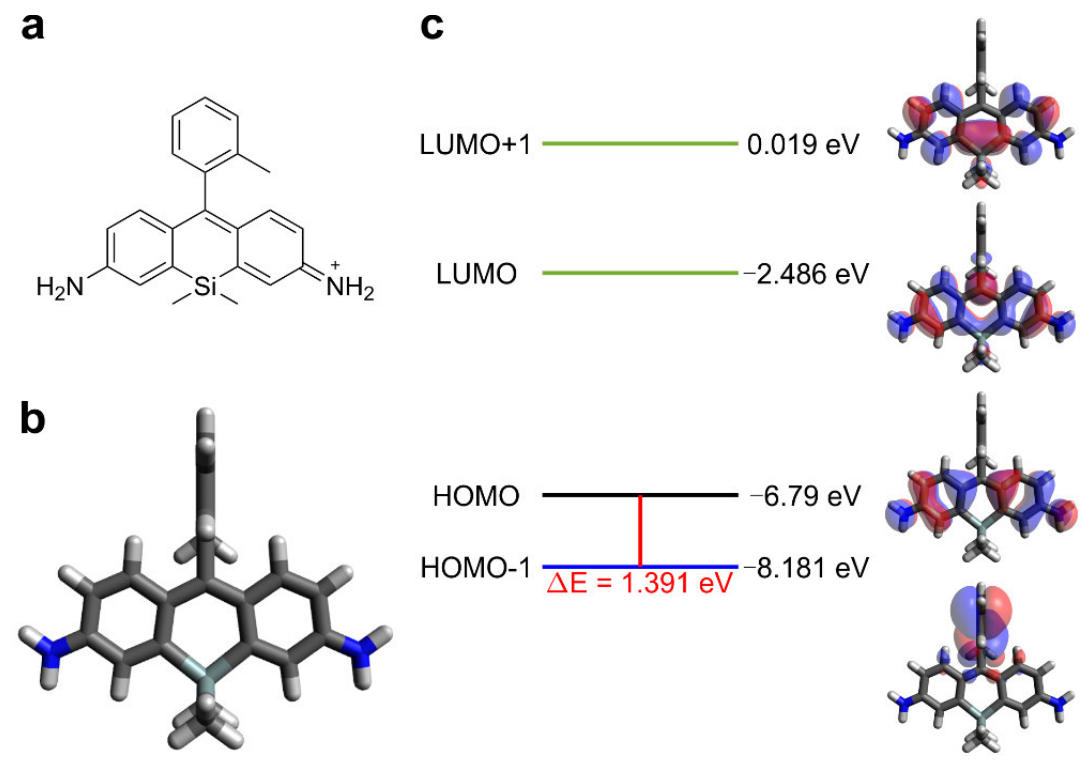

Figure S135. a) Molecular structure, b) optimized structure, c) frontier molecular orbitals and corresponding energy levels, and calculated $\triangle \mathrm{E}$ of R13 in the ground state in PBS at M062X/Def2SVP level.

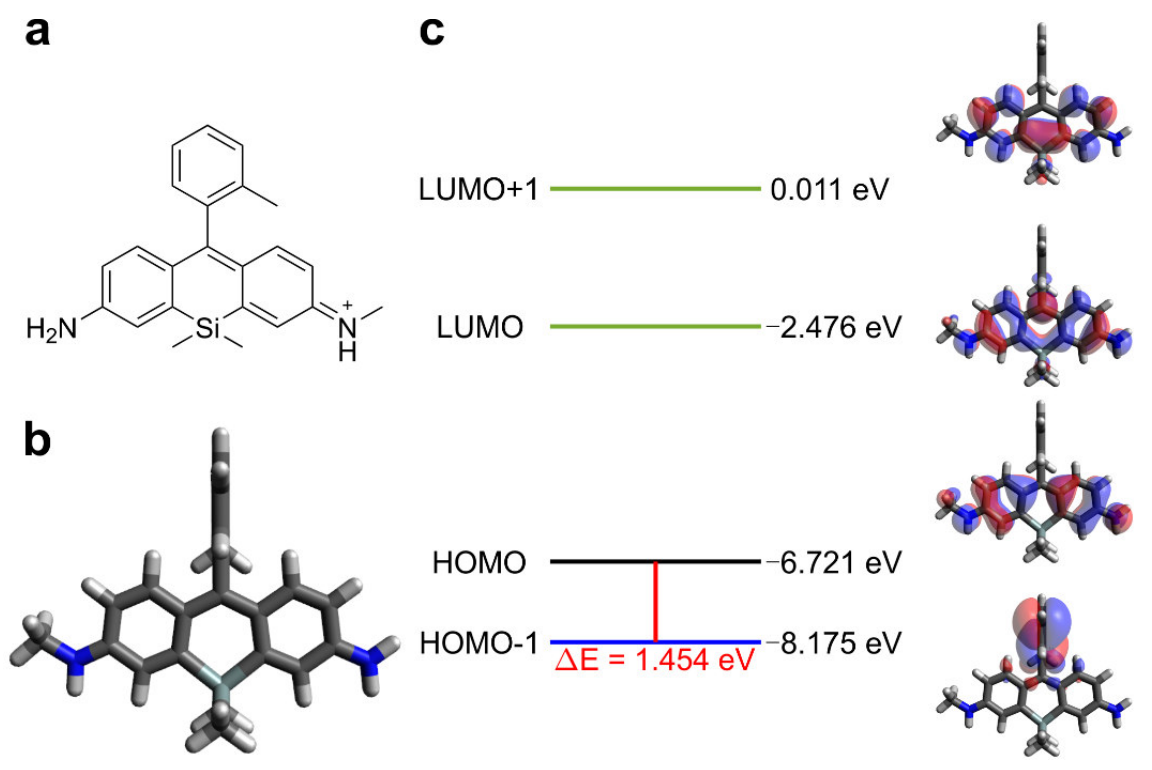

Figure S136. a) Molecular structure, b) optimized structure, c) frontier molecular orbitals and corresponding energy levels, and calculated $\Delta \mathrm{E}$ of R14 in the ground state in PBS at M062X/Def2SVP level. 


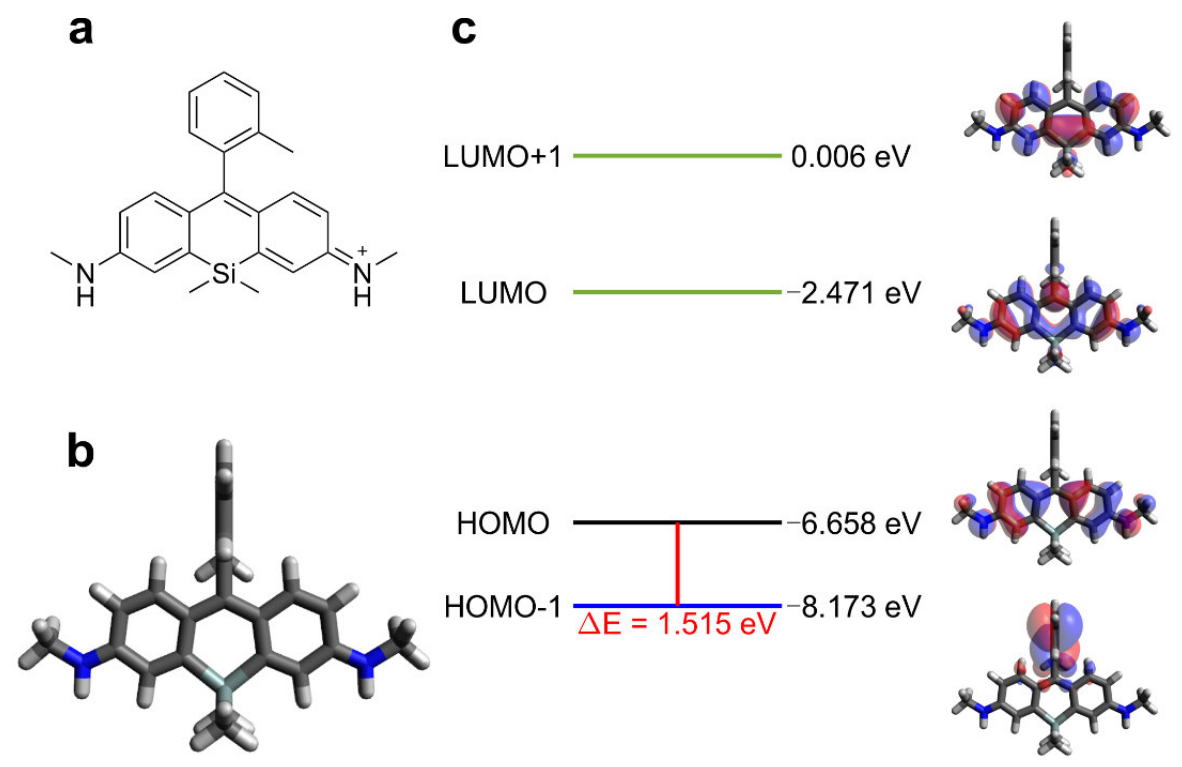

Figure S137. a) Molecular structure, b) optimized structure, c) frontier molecular orbitals and corresponding energy levels, and calculated $\triangle \mathrm{E}$ of R15 in the ground state in PBS at M062X/Def2SVP level.
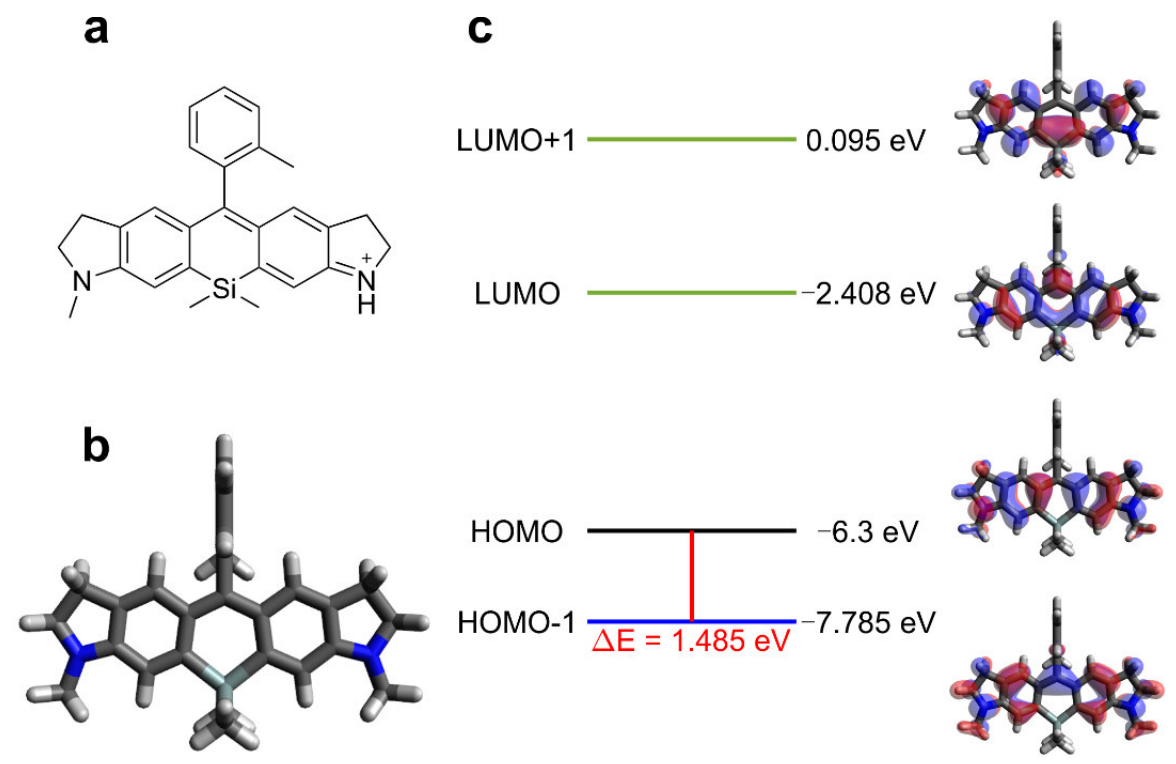

Figure S138. a) Molecular structure, b) optimized structure, c) frontier molecular orbitals and corresponding energy levels, and calculated $\Delta \mathrm{E}$ of R16 in the ground state in PBS at M062X/Def2SVP level. 
a<smiles></smiles>

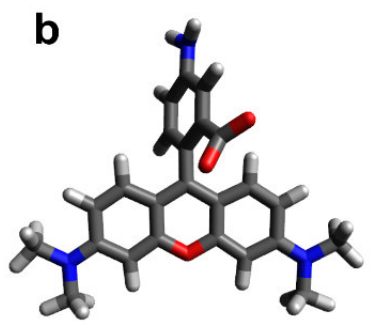

C

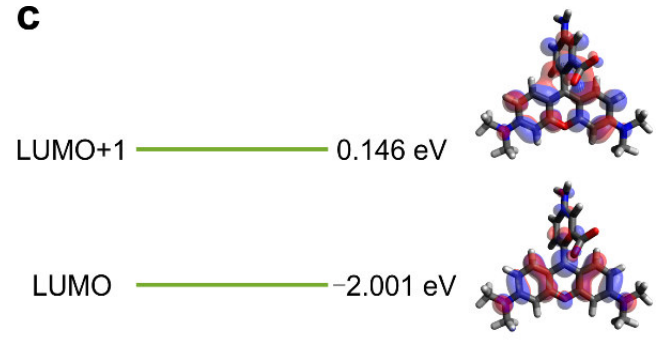

HOMO $-6.652 \mathrm{eV}$

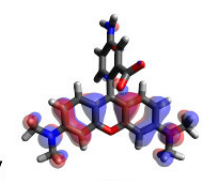
HOMO-1 $\frac{T}{\Delta \mathrm{E}=0.205 \mathrm{eV}}-6.857 \mathrm{eV}$

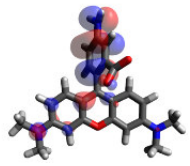

Figure S139. a) Molecular structure, b) optimized structure, c) frontier molecular orbitals and corresponding energy levels, and calculated $\Delta \mathrm{E}$ of R17 in the ground state in water at M062X/Def2SVP level.
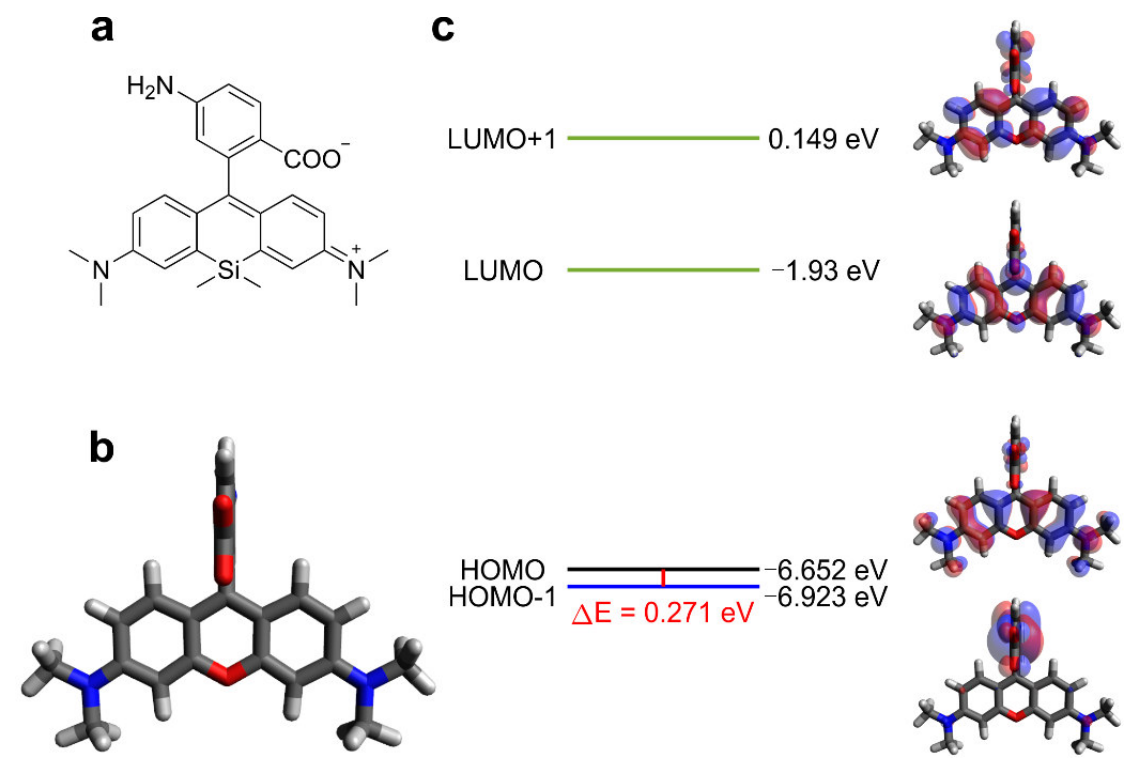

Figure S140. a) Molecular structure, b) optimized structure, c) frontier molecular orbitals and corresponding energy levels, and calculated $\Delta \mathrm{E}$ of R18 in the ground state in water at M062X/Def2SVP level. 


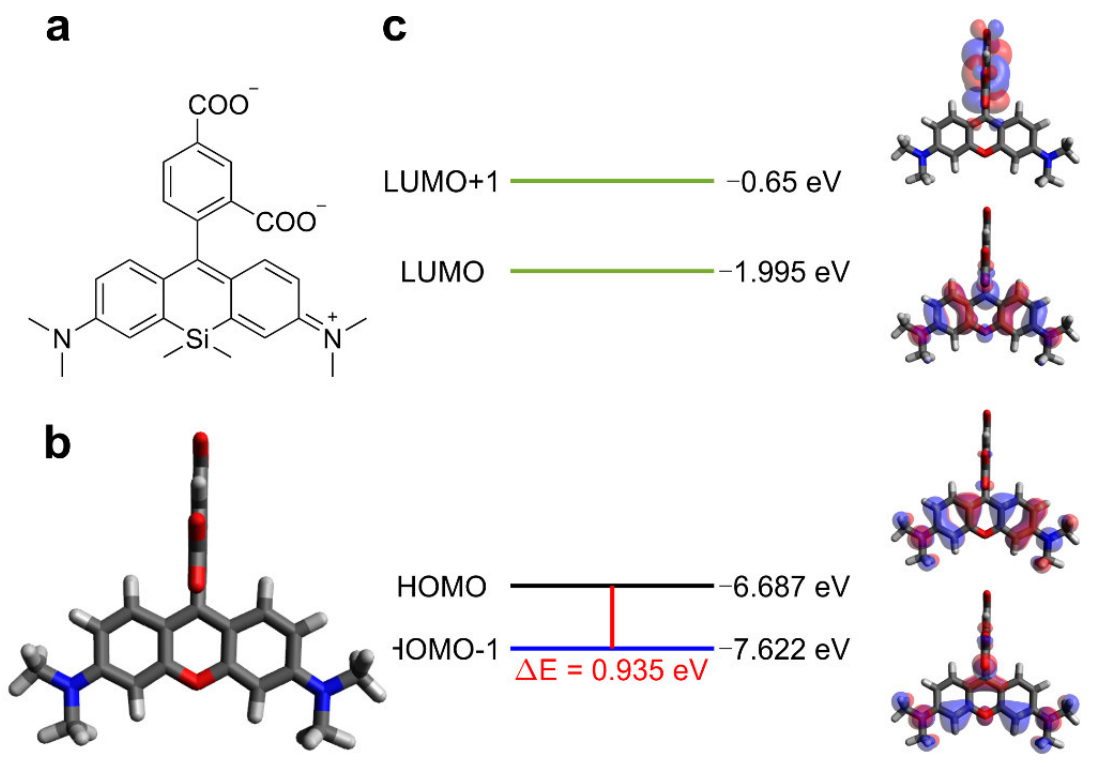

Figure S141. a) Molecular structure, b) optimized structure, c) frontier molecular orbitals and corresponding energy levels, and calculated $\Delta \mathrm{E}$ of R19 in the ground state in water at M062X/Def2SVP level.<smiles>CN(C)c1ccc2c(c1)[Si](C)(C)C1=CC(=[N+](C)C)C=CC1=C2c1cc(C(=O)[O-])ccc1C(=O)[O-]</smiles>
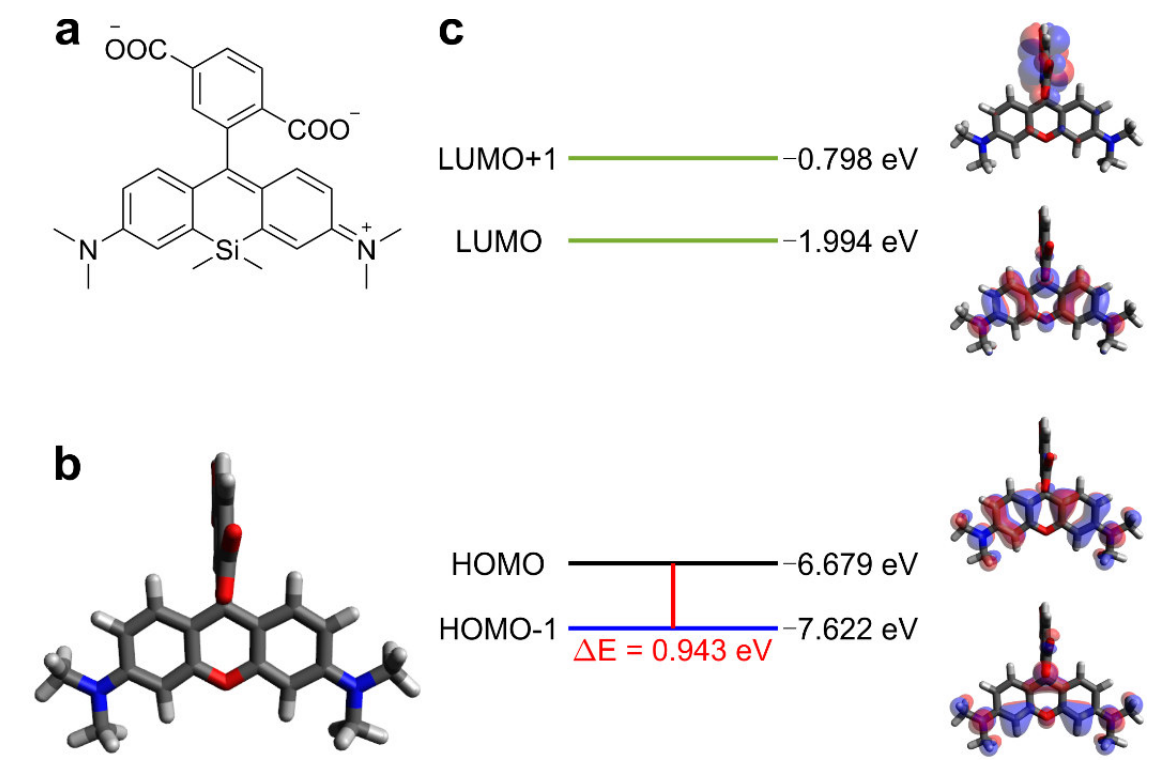

Figure S142. a) Molecular structure, b) optimized structure, c) frontier molecular orbitals and corresponding energy levels, and calculated $\Delta \mathrm{E}$ of R20 in the ground state in water at M062X/Def2SVP level. 
<smiles>C[Si]1(C)C2=C3CCC[n+]4c3c(cc1c4-c1ccccc1C(=O)[O-])CCC2</smiles>

C
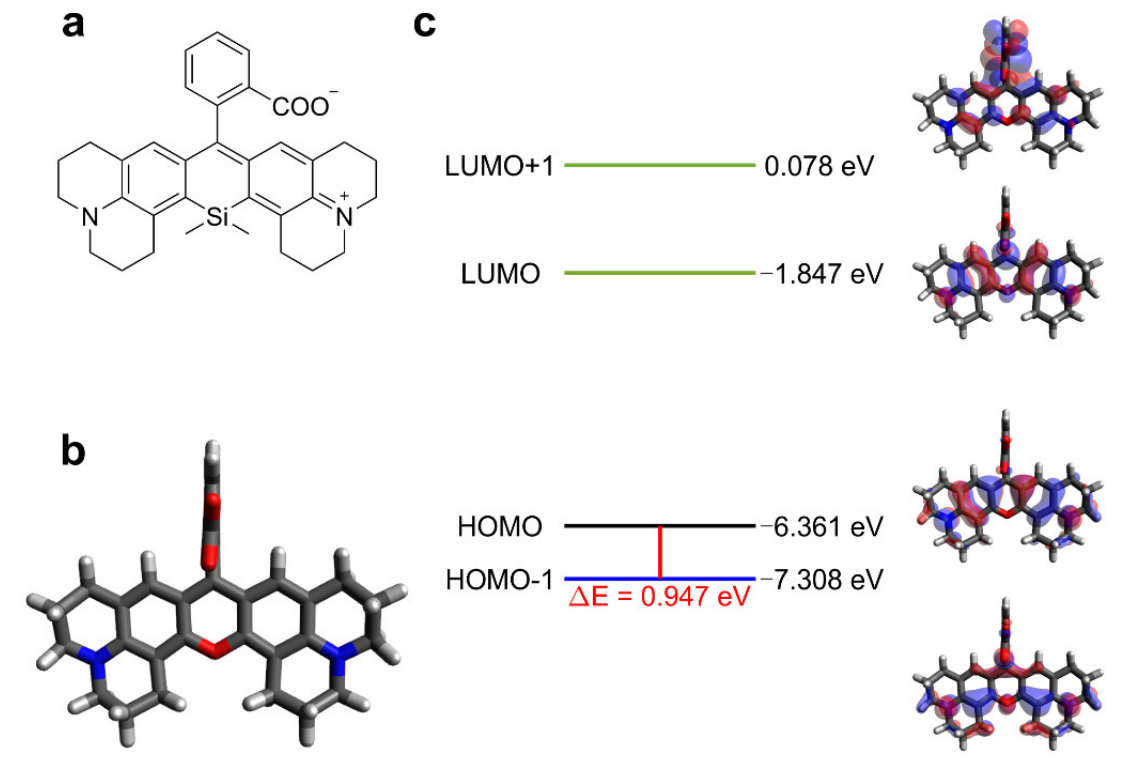

Figure S143. a) Molecular structure, b) optimized structure, c) frontier molecular orbitals and corresponding energy levels, and calculated $\Delta \mathrm{E}$ of R21 in the ground state in water at M062X/Def2SVP level.
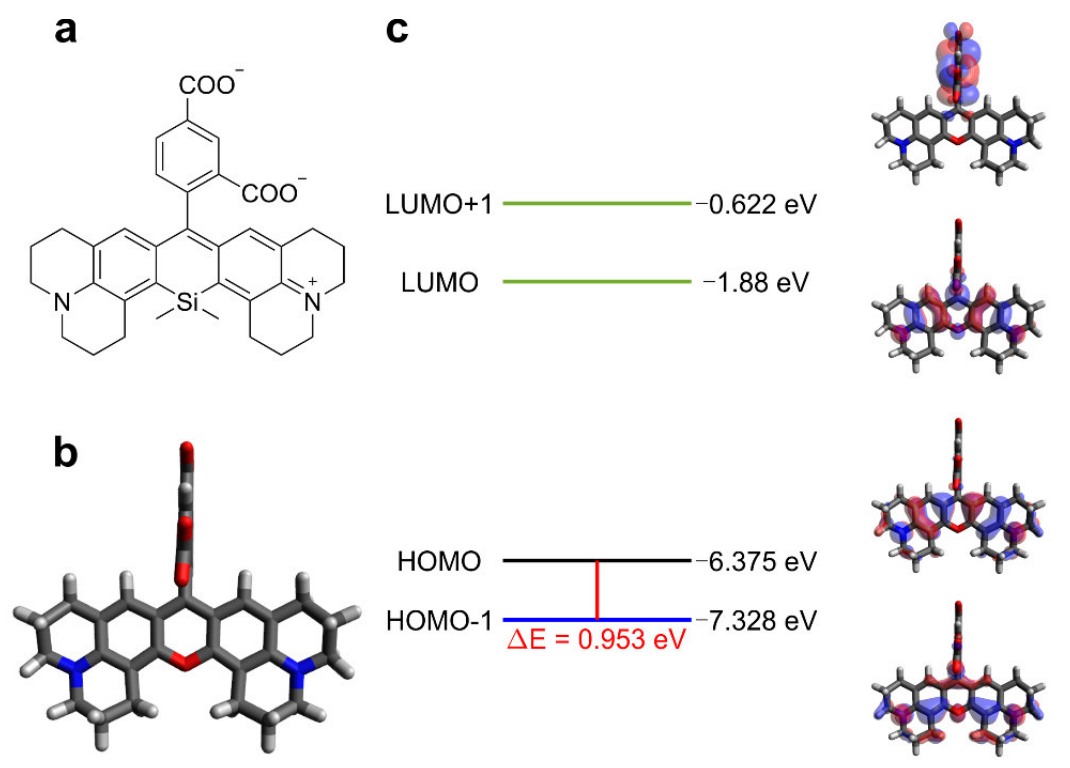

Figure S144. a) Molecular structure, b) optimized structure, c) frontier molecular orbitals and corresponding energy levels, and calculated $\Delta \mathrm{E}$ of R22 in the ground state in water at M062X/Def2SVP level. 

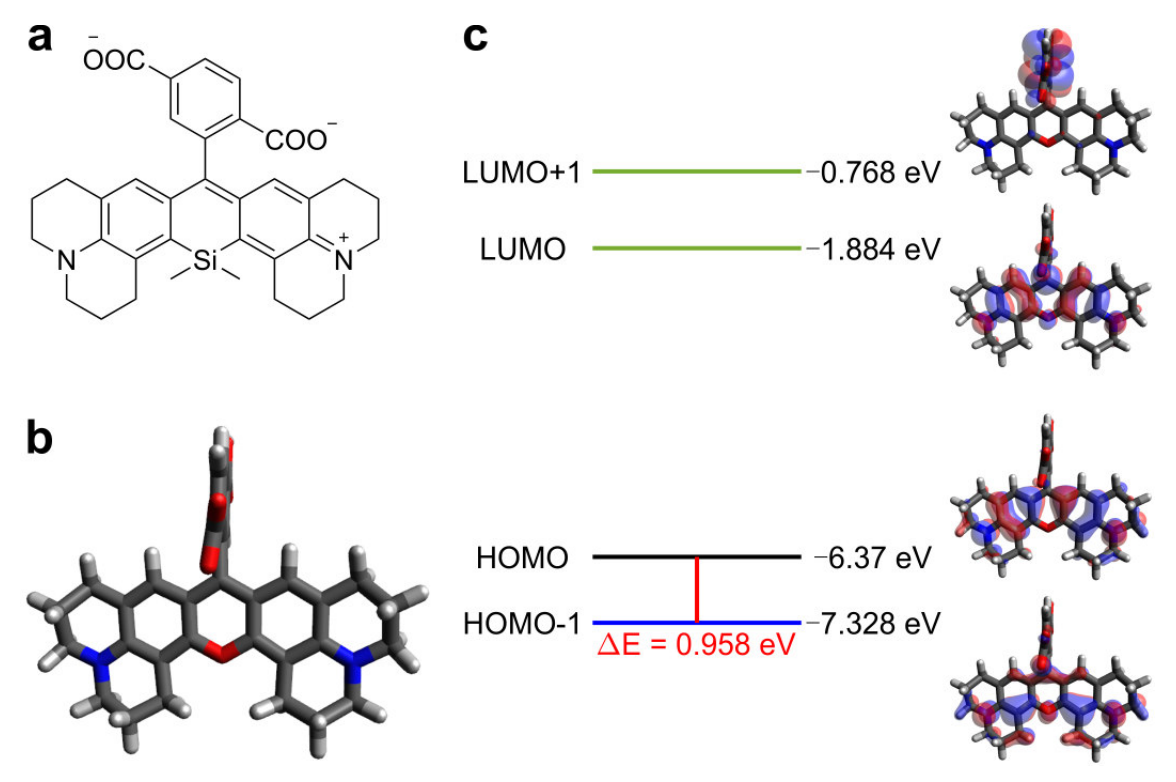

Figure S145. a) Molecular structure, b) optimized structure, c) frontier molecular orbitals and corresponding energy levels, and calculated $\Delta \mathrm{E}$ of R23 in the ground state in water at M062X/Def2SVP level.

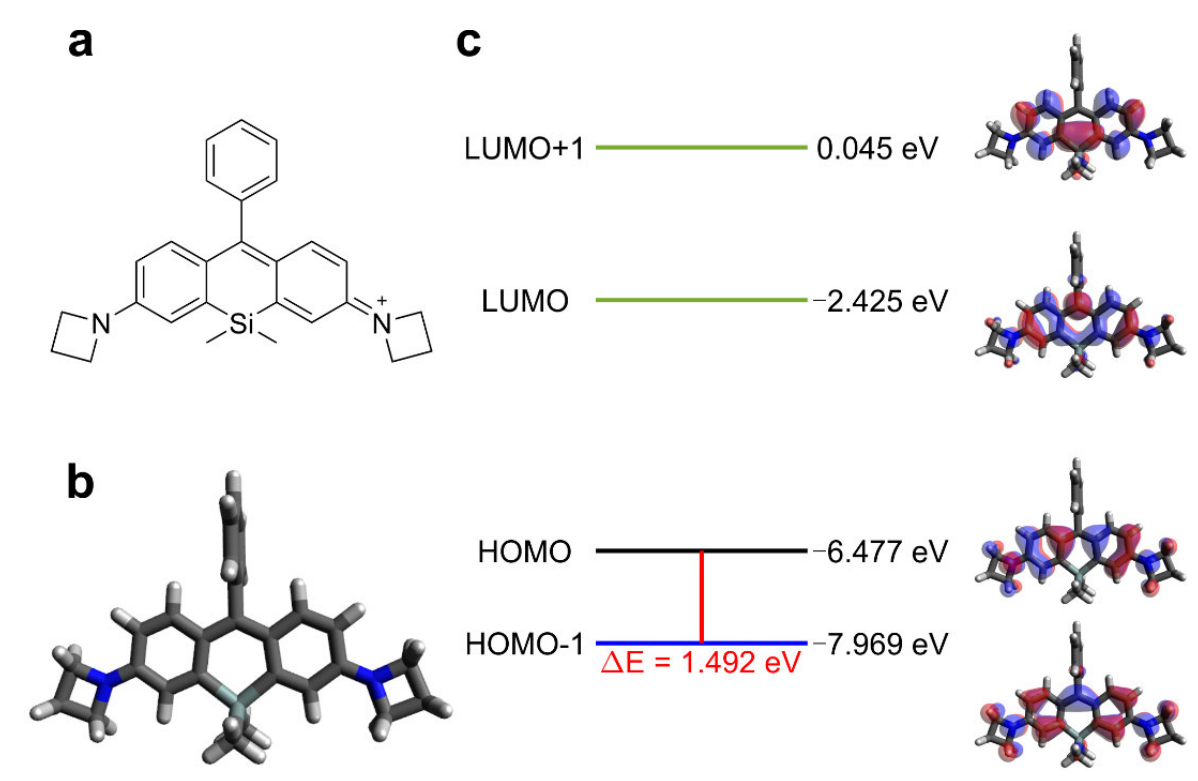

Figure S146. a) Molecular structure, b) optimized structure, c) frontier molecular orbitals and corresponding energy levels, and calculated $\triangle \mathrm{E}$ of R24 in the ground state in PBS at M062X/Def2SVP level. 

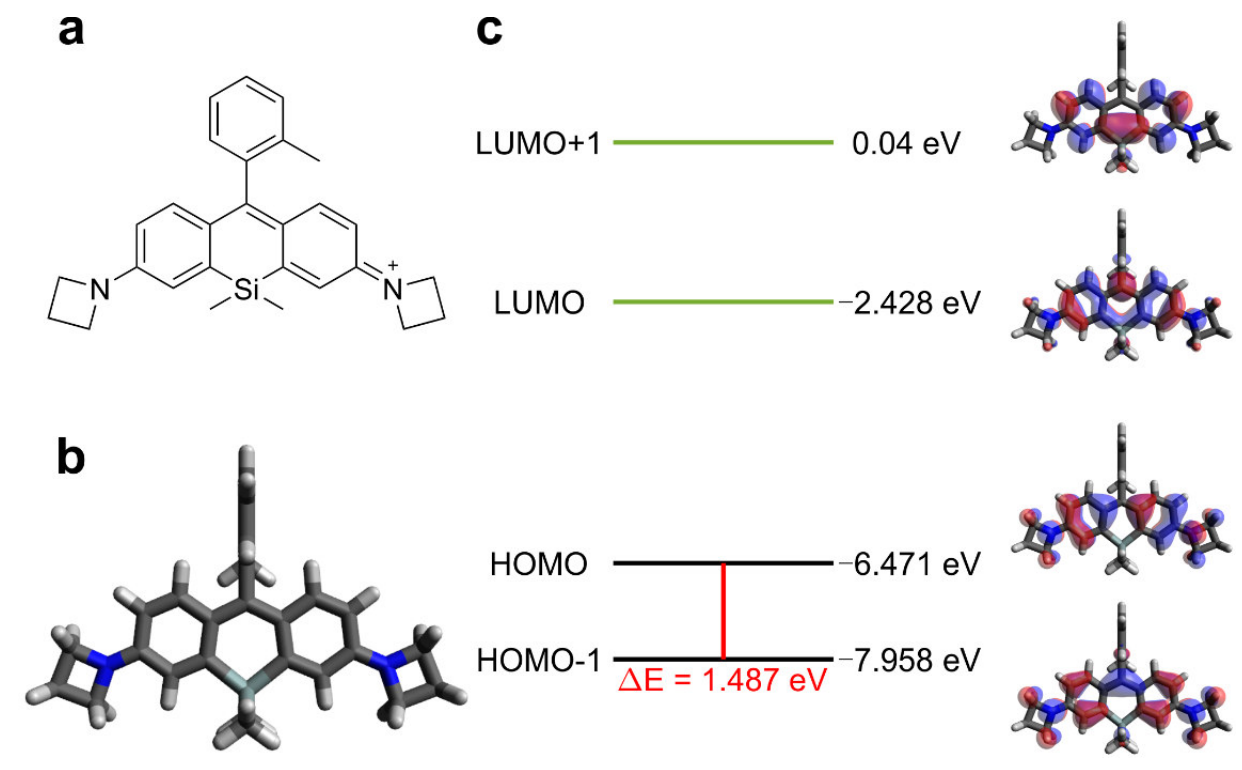

Figure S147. a) Molecular structure, b) optimized structure, c) frontier molecular orbitals and corresponding energy levels, and calculated $\triangle \mathrm{E}$ of R25 in the ground state in PBS at M062X/Def2SVP level.

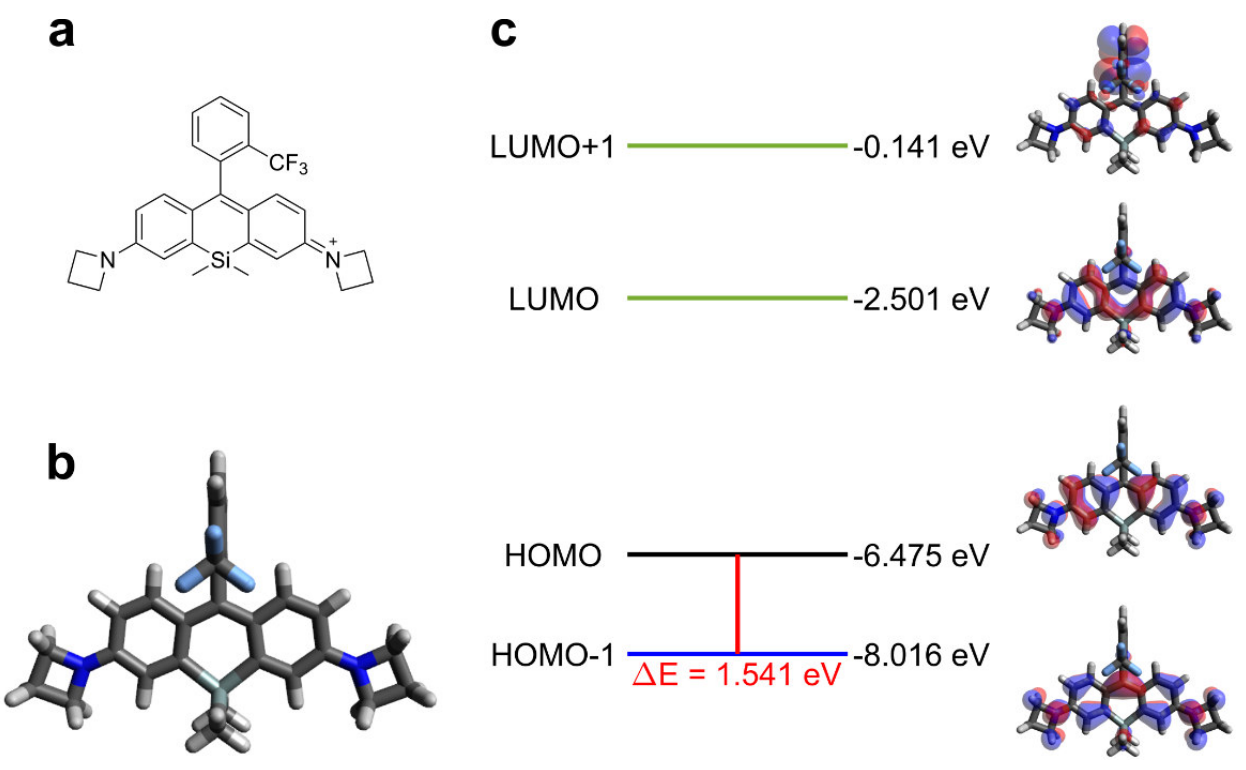

Figure S148. a) Molecular structure, b) optimized structure, c) frontier molecular orbitals and corresponding energy levels, and calculated $\Delta \mathrm{E}$ of R26 in the ground state in PBS at M062X/Def2SVP level. 
a<smiles></smiles>

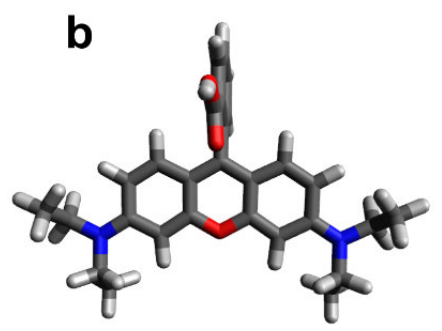

C
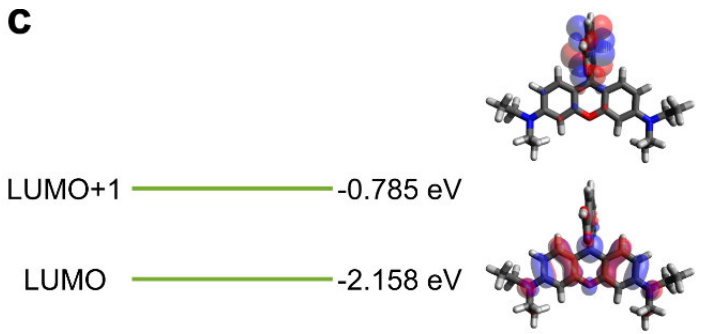

HOMO

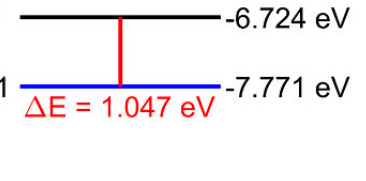

Figure S149. a) Molecular structure, b) optimized structure, c) frontier molecular orbitals and corresponding energy levels, and calculated $\Delta \mathrm{E}$ of R27 in the ground state in ethanol at M062X/Def2SVP level.

a<smiles></smiles>

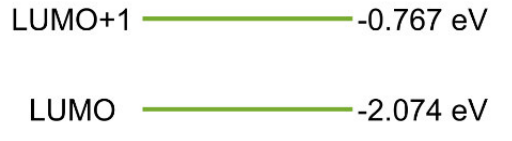

C

HOMO
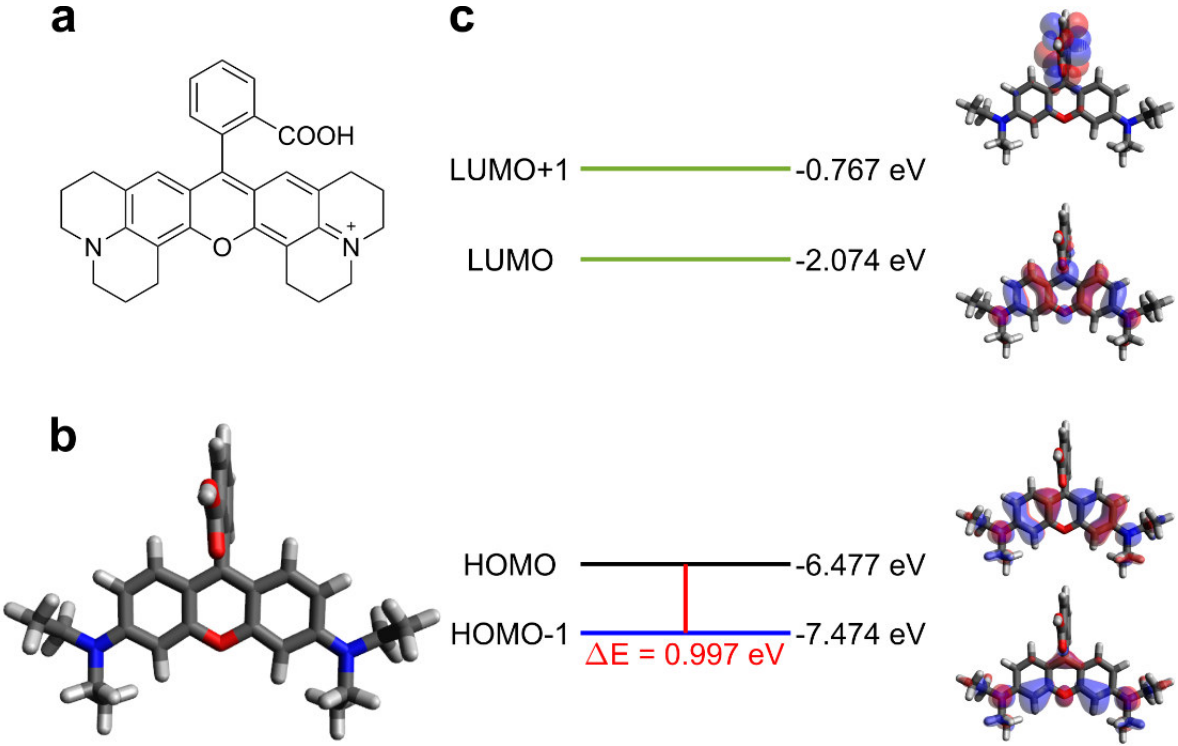

HOMO-1 $\frac{}{\Delta E=0.997 \mathrm{eV}}-7.474 \mathrm{eV}$

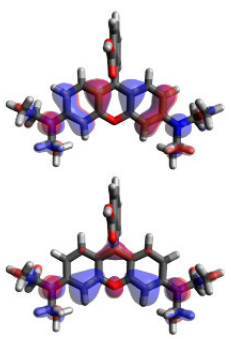

Figure S150. a) Molecular structure, b) optimized structure, c) frontier molecular orbitals and corresponding energy levels, and calculated $\Delta \mathrm{E}$ of R28 in the ground state in ethanol at M062X/Def2SVP level. 


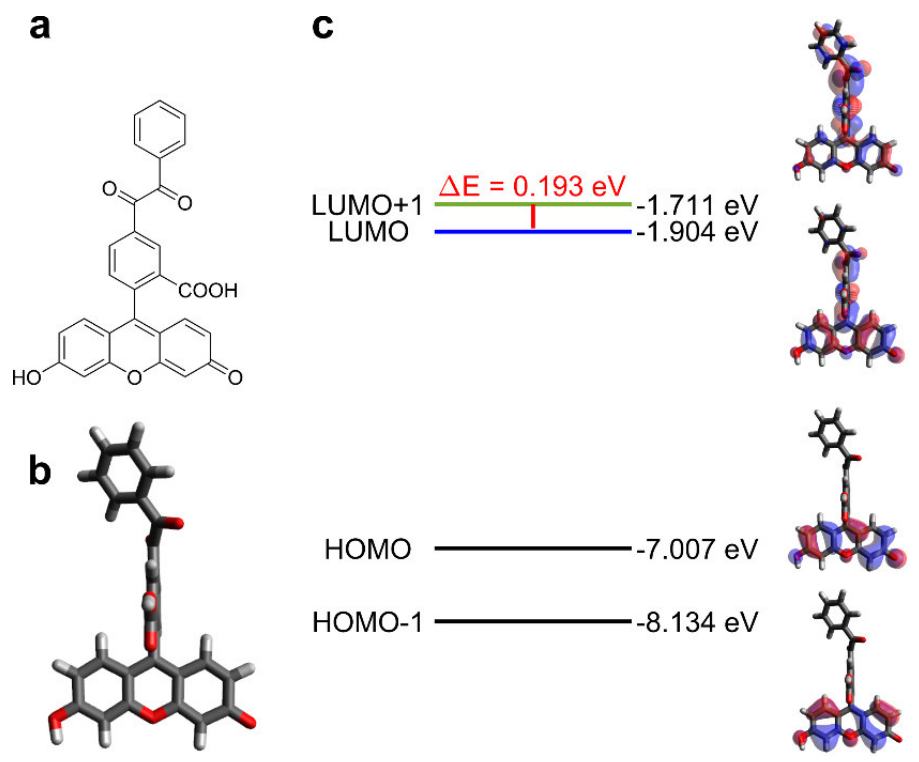

Figure S151. a) Molecular structure, b) optimized structure, c) frontier molecular orbitals and corresponding energy levels, and calculated $\triangle \mathrm{E}$ of F1 in the ground state in PBS at M062X/Def2SVP level.

a

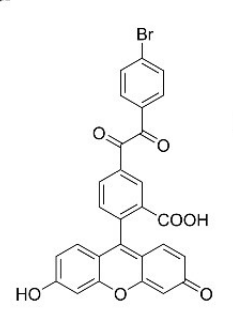

C LUMO+1 $\underline{\text { LUMO }=0.158 \mathrm{eV}}-1.757 \mathrm{eV}$
$=-1.915 \mathrm{eV}$

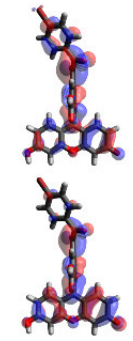

b

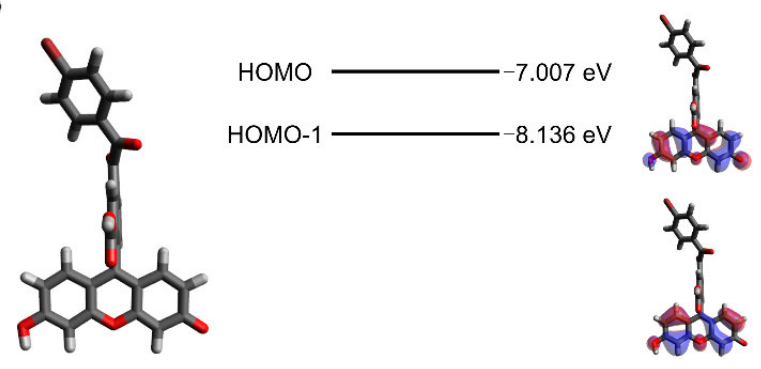

Figure S152. a) Molecular structure, b) optimized structure, c) frontier molecular orbitals and corresponding energy levels, and calculated $\triangle \mathrm{E}$ of $\mathbf{F 2}$ in the ground state in PBS at M062X/Def2SVP level. 
a

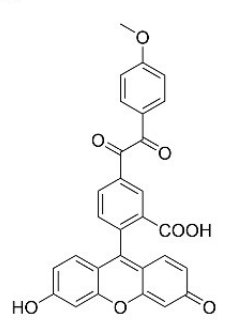

b

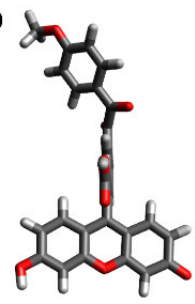

C

LUMO+1 $\Delta \mathrm{E}=0.261 \mathrm{eV}-1.636 \mathrm{eV}$
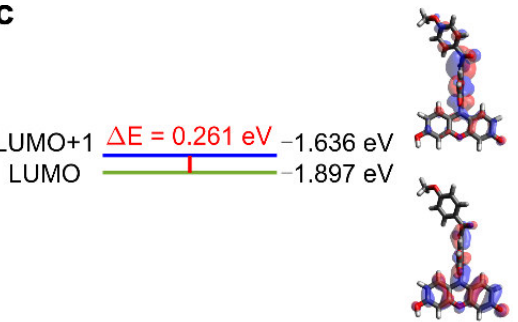

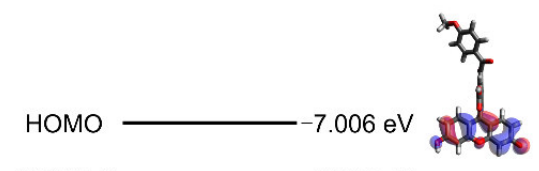

HOMO-1 $-7.896 \mathrm{eV}$

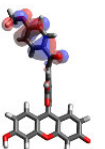

Figure S153. a) Molecular structure, b) optimized structure, c) frontier molecular orbitals and corresponding energy levels, and calculated $\triangle \mathrm{E}$ of F3 in the ground state in PBS at M062X/Def2SVP level.

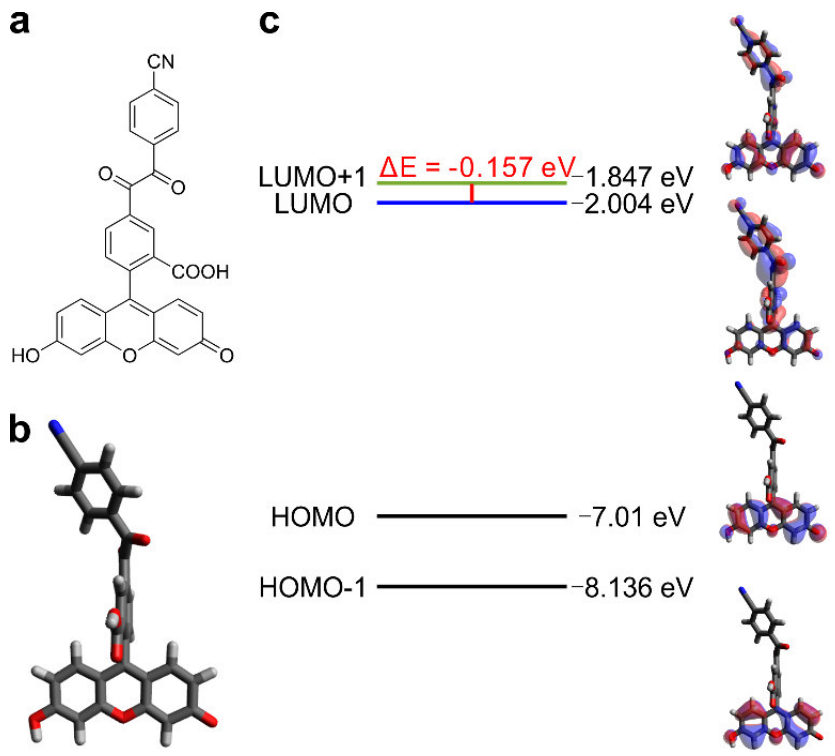

Figure S154. a) Molecular structure, b) optimized structure, c) frontier molecular orbitals and corresponding energy levels, and calculated $\Delta \mathrm{E}$ of F4 in the ground state in PBS at M062X/Def2SVP level. 


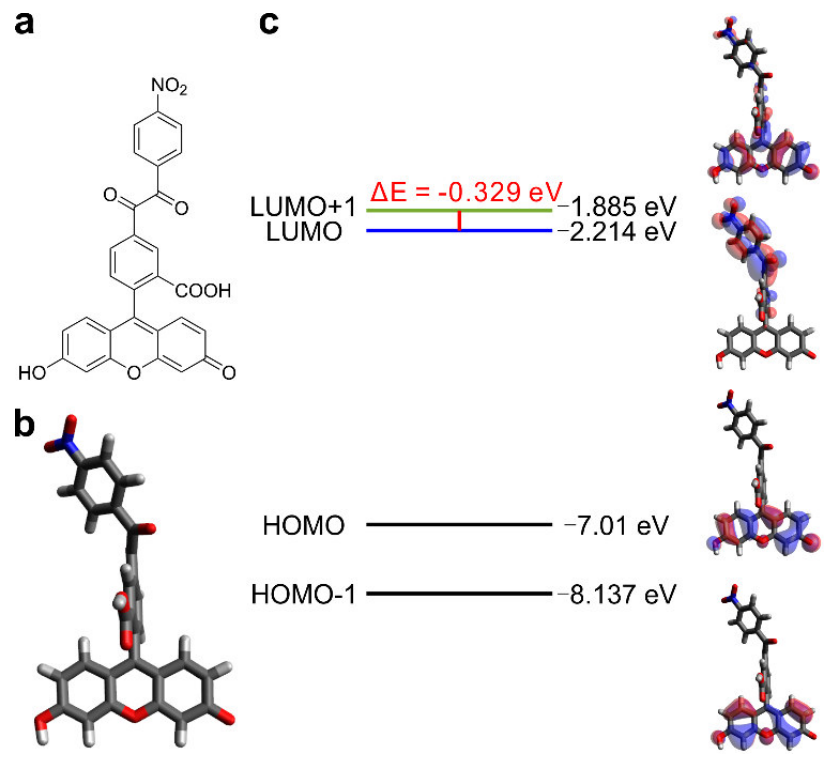

Figure S155. a) Molecular structure, b) optimized structure, c) frontier molecular orbitals and corresponding energy levels, and calculated $\triangle \mathrm{E}$ of F5 in the ground state in PBS at M062X/Def2SVP level.
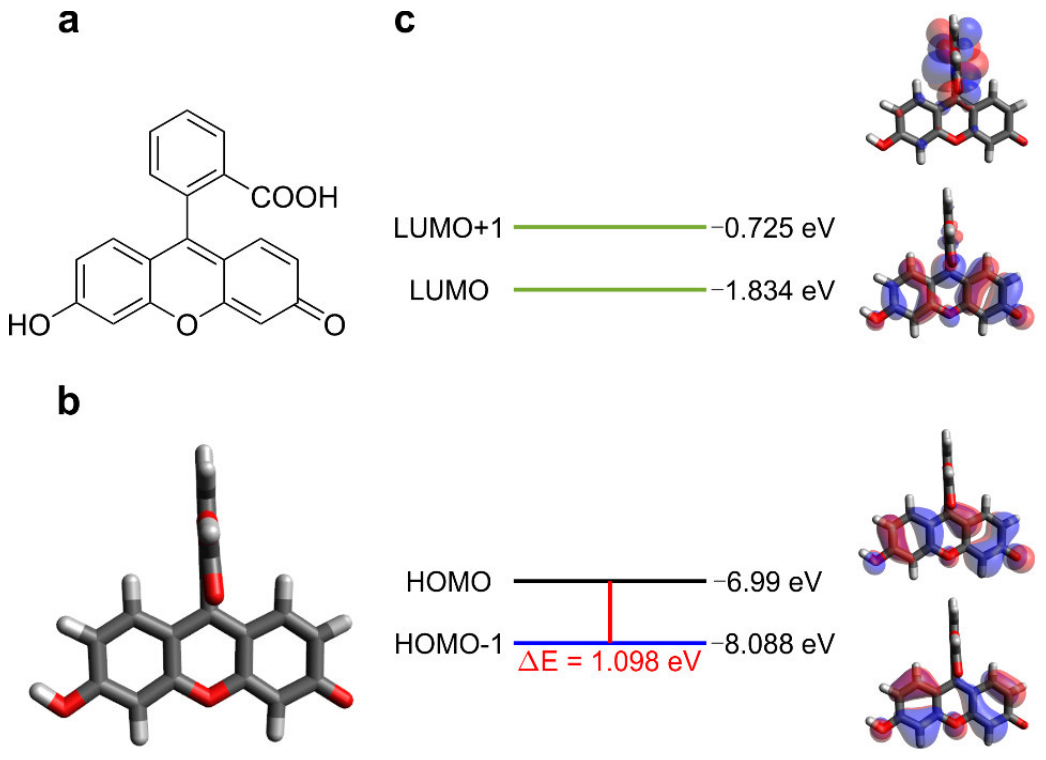

Figure S156. a) Molecular structure, b) optimized structure, c) frontier molecular orbitals and corresponding energy levels, and calculated $\Delta \mathrm{E}$ of F6 in the ground state in water at M062X/Def2SVP level. 


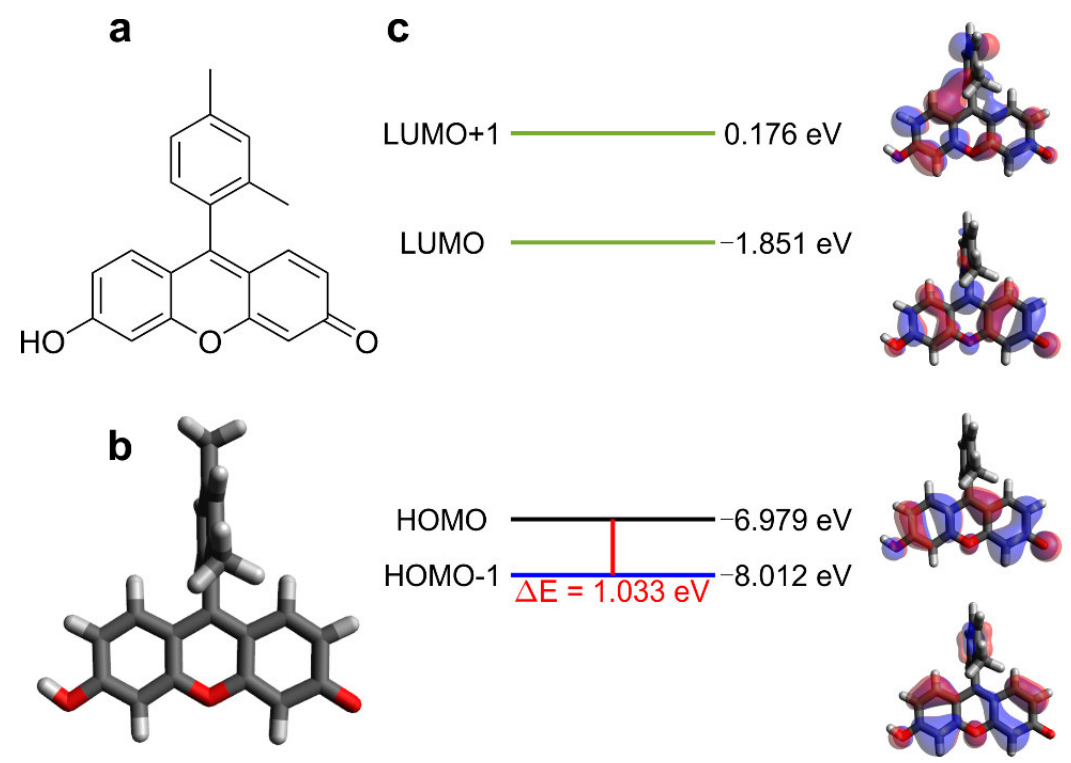

Figure S157. a) Molecular structure, b) optimized structure, c) frontier molecular orbitals and corresponding energy levels, and calculated $\Delta \mathrm{E}$ of $\mathbf{F 7}$ in the ground state in water at M062X/Def2SVP level.
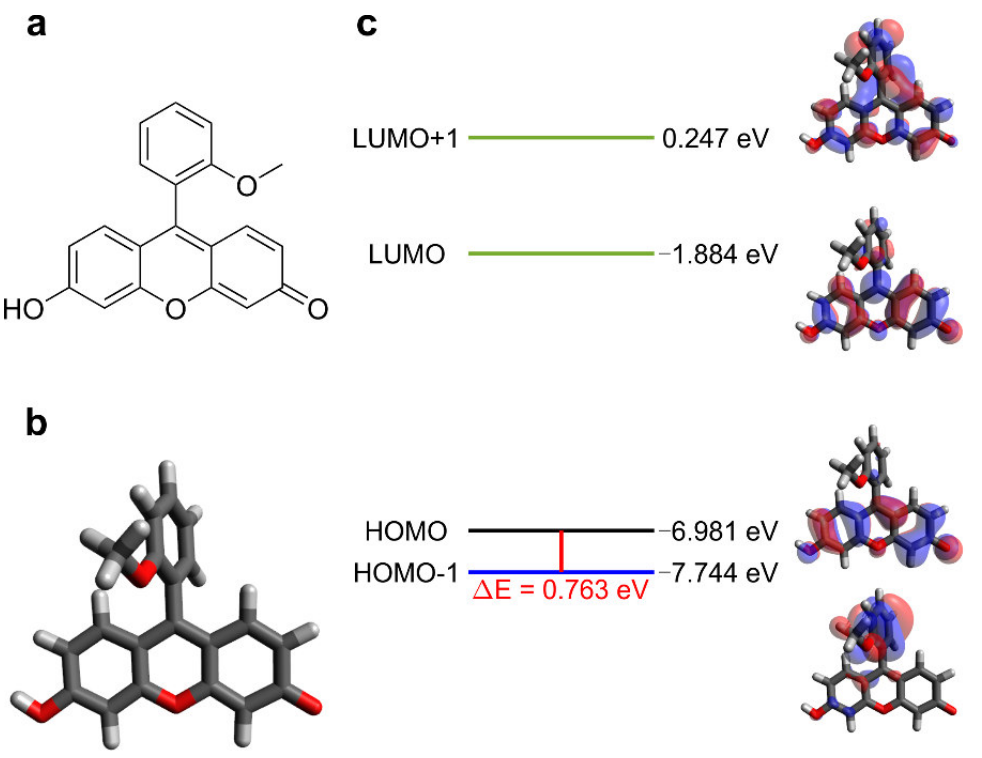

Figure S158. a) Molecular structure, b) optimized structure, c) frontier molecular orbitals and corresponding energy levels, and calculated $\Delta \mathrm{E}$ of F8 in the ground state in water at M062X/Def2SVP level. 
<smiles>Cc1ccccc1-c1c2ccc(=O)cc-2oc2cc(O)ccc12</smiles>

C

LUMO+1 $-0.171 \mathrm{eV}$

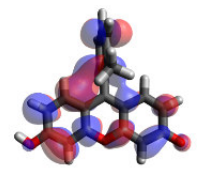

LUMO $-1.866 \mathrm{eV}$

b
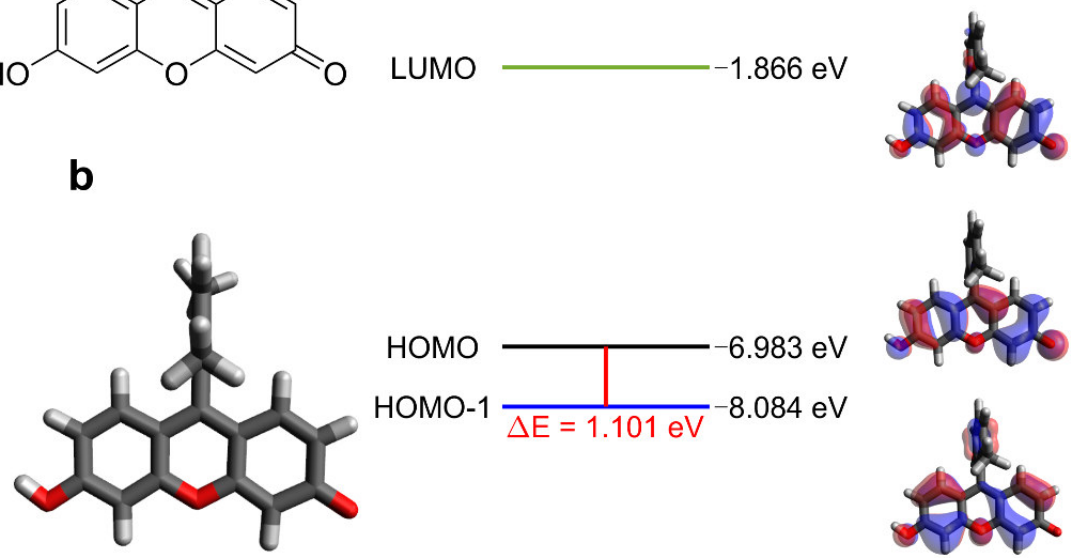

Figure S159. a) Molecular structure, b) optimized structure, c) frontier molecular orbitals and corresponding energy levels, and calculated $\Delta \mathrm{E}$ of F9 in the ground state in water at M062X/Def2SVP level.

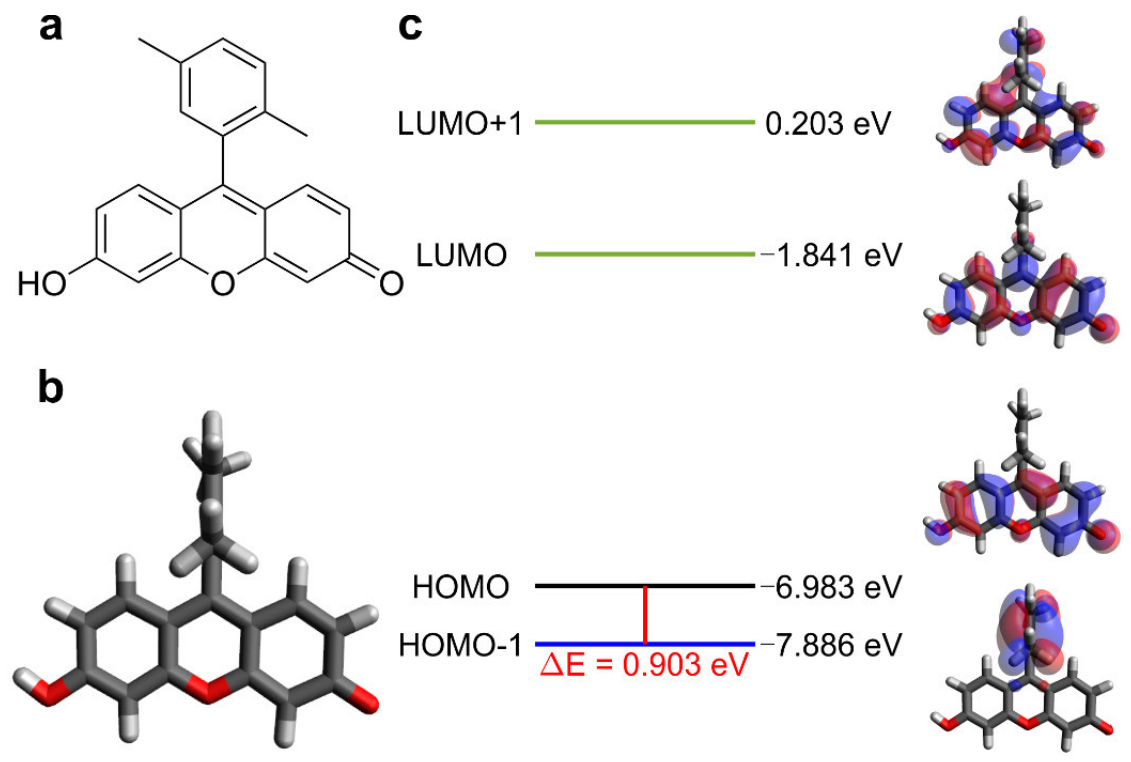

Figure S160. a) Molecular structure, b) optimized structure, c) frontier molecular orbitals and corresponding energy levels, and calculated $\Delta \mathrm{E}$ of F10 in the ground state in water at M062X/Def2SVP level. 

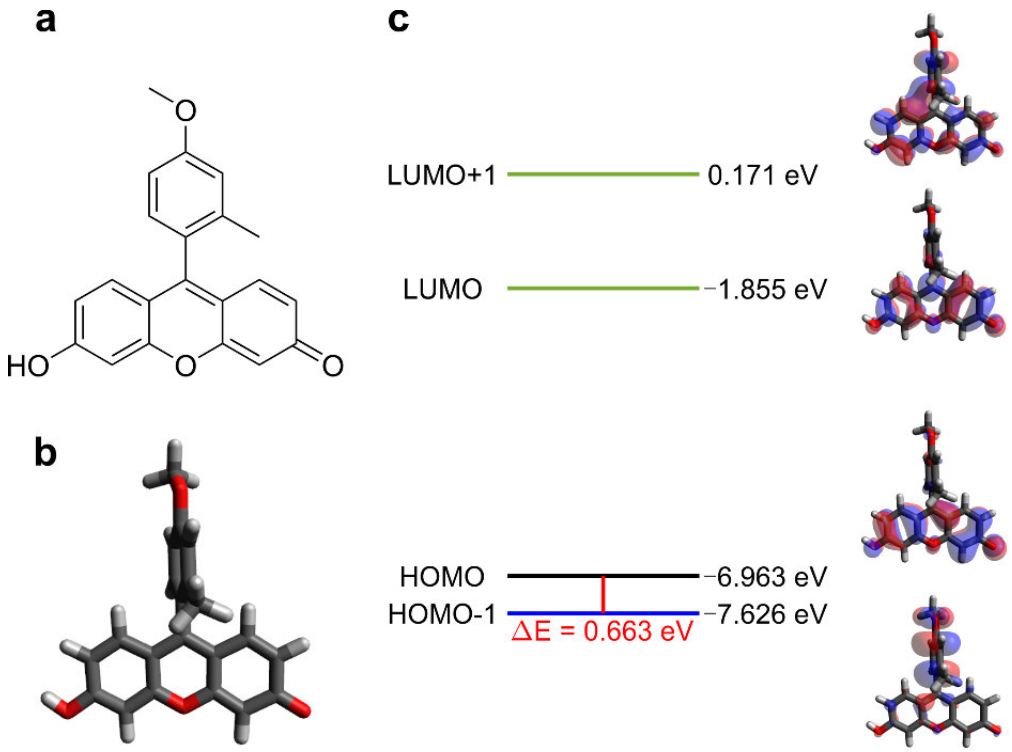

Figure S161. a) Molecular structure, b) optimized structure, c) frontier molecular orbitals and corresponding energy levels, and calculated $\Delta \mathrm{E}$ of F11 in the ground state in water at M062X/Def2SVP level.
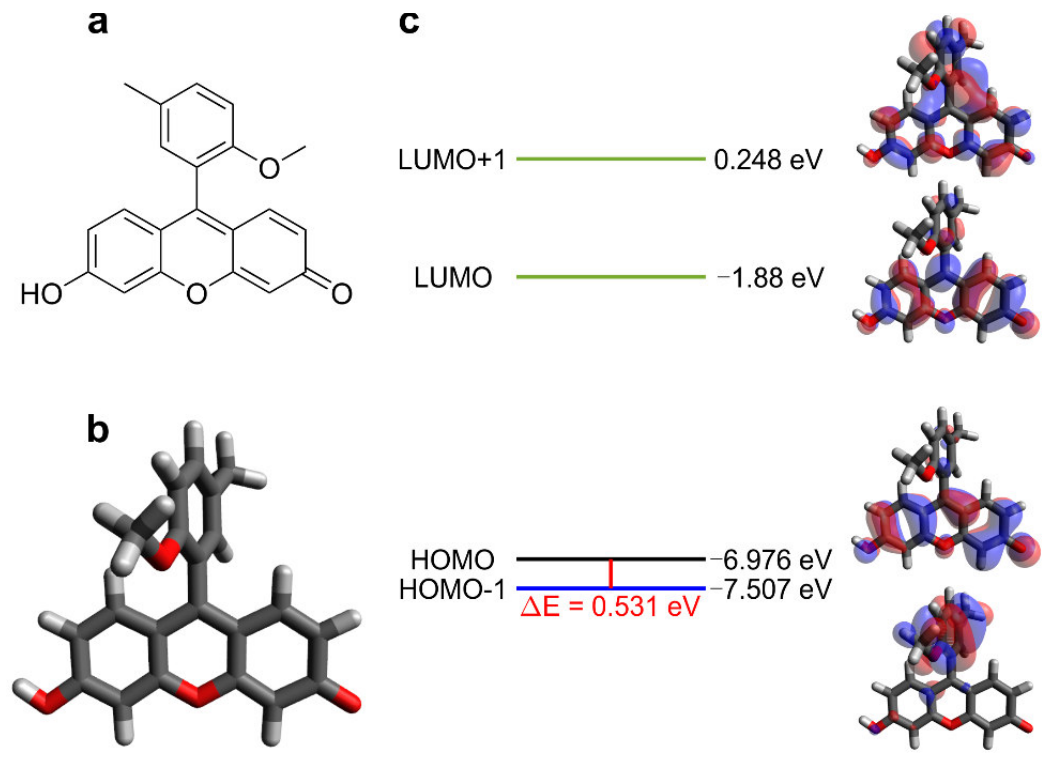

Figure S162. a) Molecular structure, b) optimized structure, c) frontier molecular orbitals and corresponding energy levels, and calculated $\Delta \mathrm{E}$ of F12 in the ground state in water at M062X/Def2SVP level. 


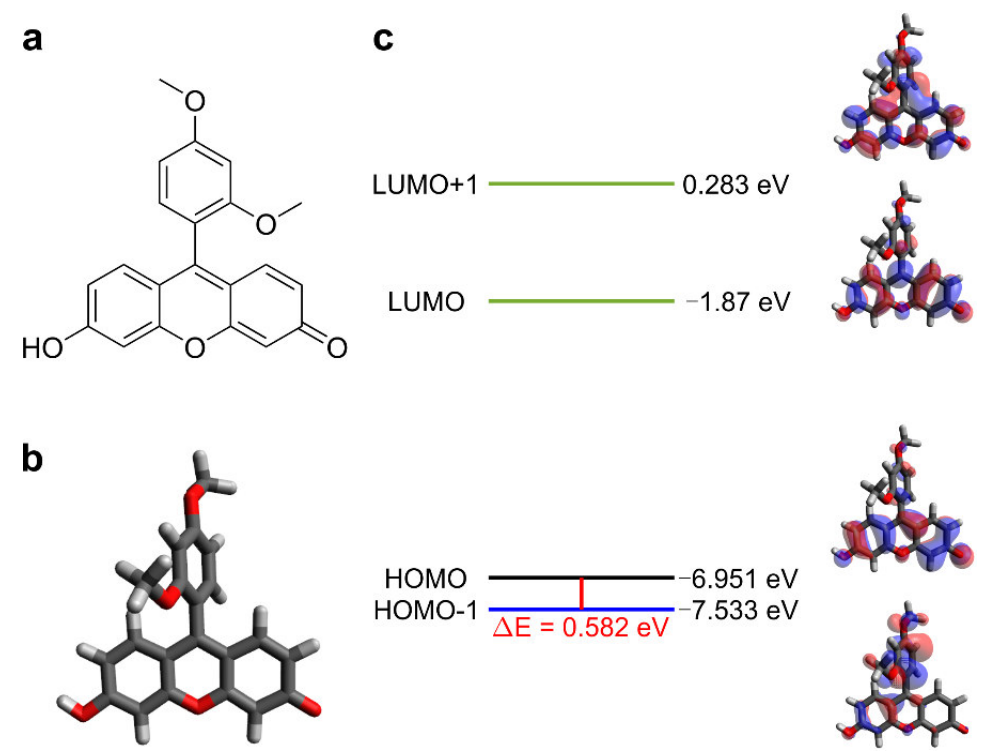

Figure S163. a) Molecular structure, b) optimized structure, c) frontier molecular orbitals and corresponding energy levels, and calculated $\Delta \mathrm{E}$ of F13 in the ground state in water at M062X/Def2SVP level.
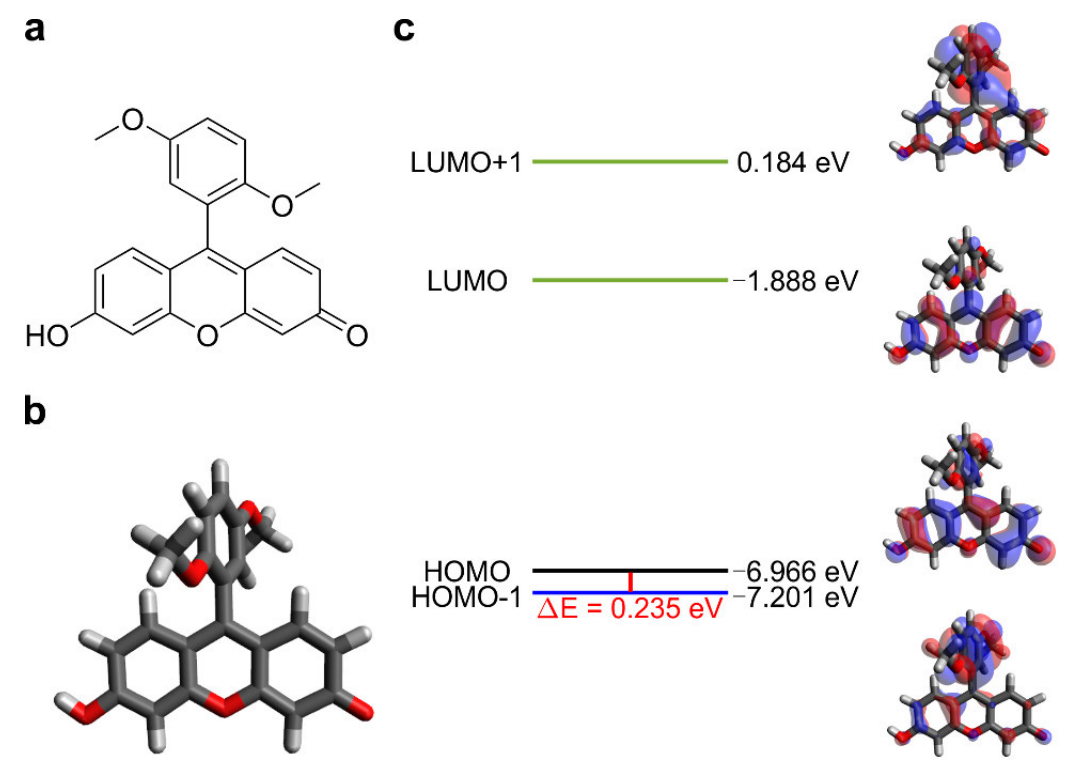

Figure S164. a) Molecular structure, b) optimized structure, c) frontier molecular orbitals and corresponding energy levels, and calculated $\Delta \mathrm{E}$ of F14 in the ground state in water at M062X/Def2SVP level. 
<smiles></smiles>

C

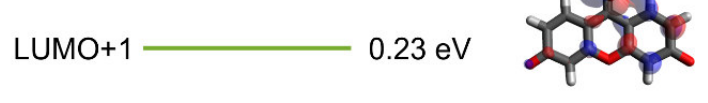

LUMO $-1.315 \mathrm{eV}$
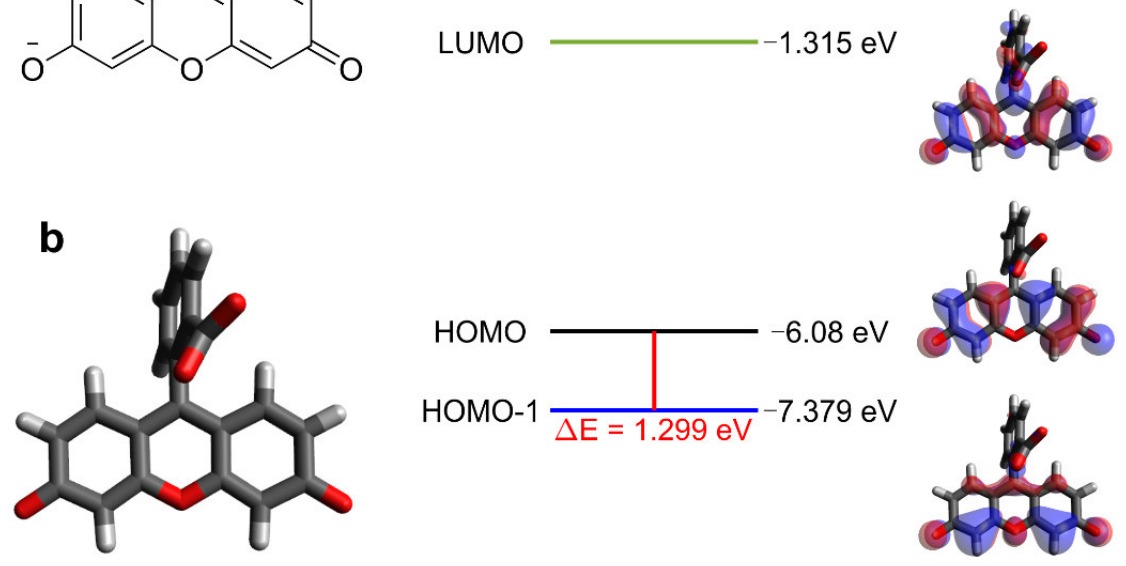

Figure S165. a) Molecular structure, b) optimized structure, c) frontier molecular orbitals and corresponding energy levels, and calculated $\Delta \mathrm{E}$ of F15 in the ground state in water at M062X/Def2SVP level.

a<smiles>Cc1ccc(-c2c3ccc(=O)cc-3oc3cc([O-])ccc23)c(C)c1</smiles>

b

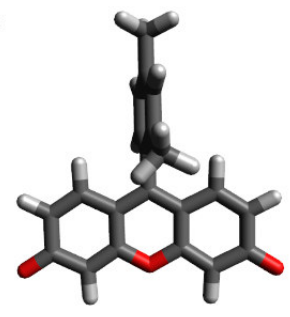

C

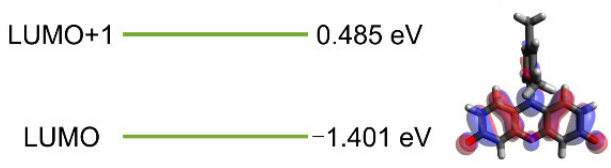

HOMO $-6.126 \mathrm{eV}$
HOMO-1 $\frac{1.329 \mathrm{eV}}{\Delta \mathrm{E}=1.455 \mathrm{eV}}$

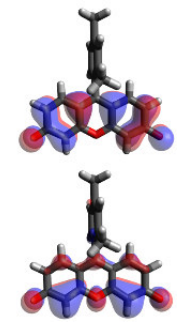

Figure S166. a) Molecular structure, b) optimized structure, c) frontier molecular orbitals and corresponding energy levels, and calculated $\Delta \mathrm{E}$ of F16 in the ground state in water at M062X/Def2SVP level. 
a<smiles></smiles>
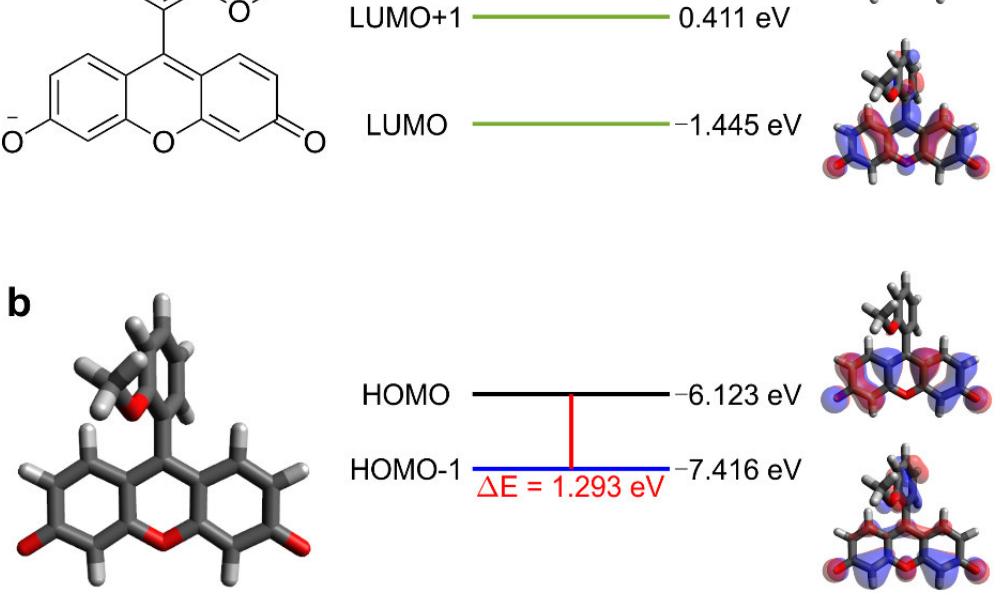

LUMO+1

LUMO $-1.445 \mathrm{eV}$

C

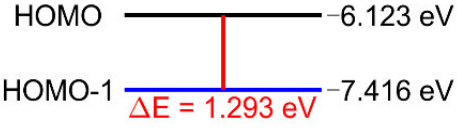

Figure S167. a) Molecular structure, b) optimized structure, c) frontier molecular orbitals and corresponding energy levels, and calculated $\Delta \mathrm{E}$ of F17 in the ground state in water at M062X/Def2SVP level.
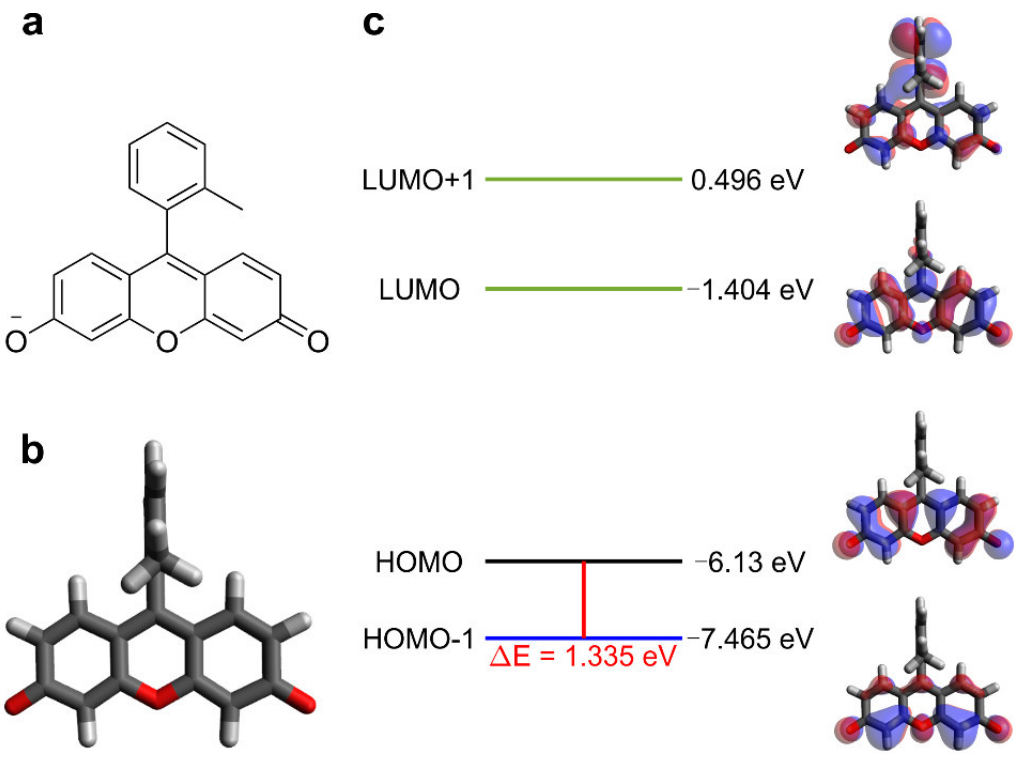

Figure S168. a) Molecular structure, b) optimized structure, c) frontier molecular orbitals and corresponding energy levels, and calculated $\Delta \mathrm{E}$ of F18 in the ground state in water at M062X/Def2SVP level. 

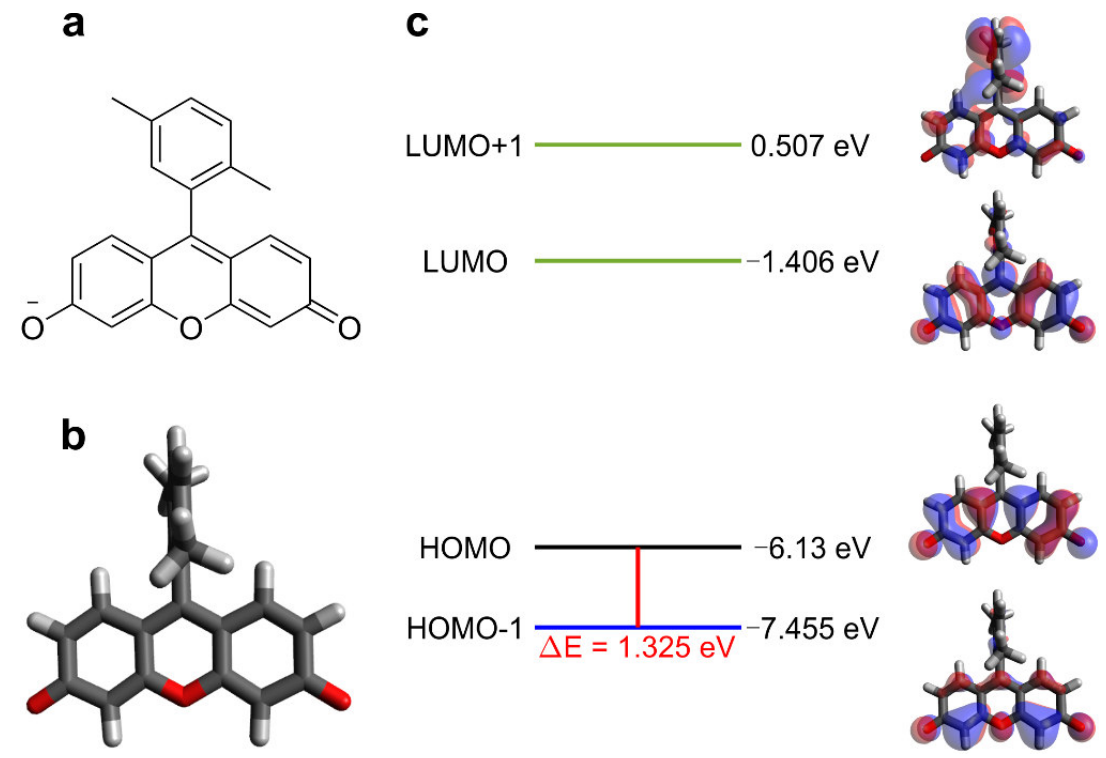

Figure S169. a) Molecular structure, b) optimized structure, c) frontier molecular orbitals and corresponding energy levels, and calculated $\Delta E$ of F19 in the ground state in water at M062X/Def2SVP level.
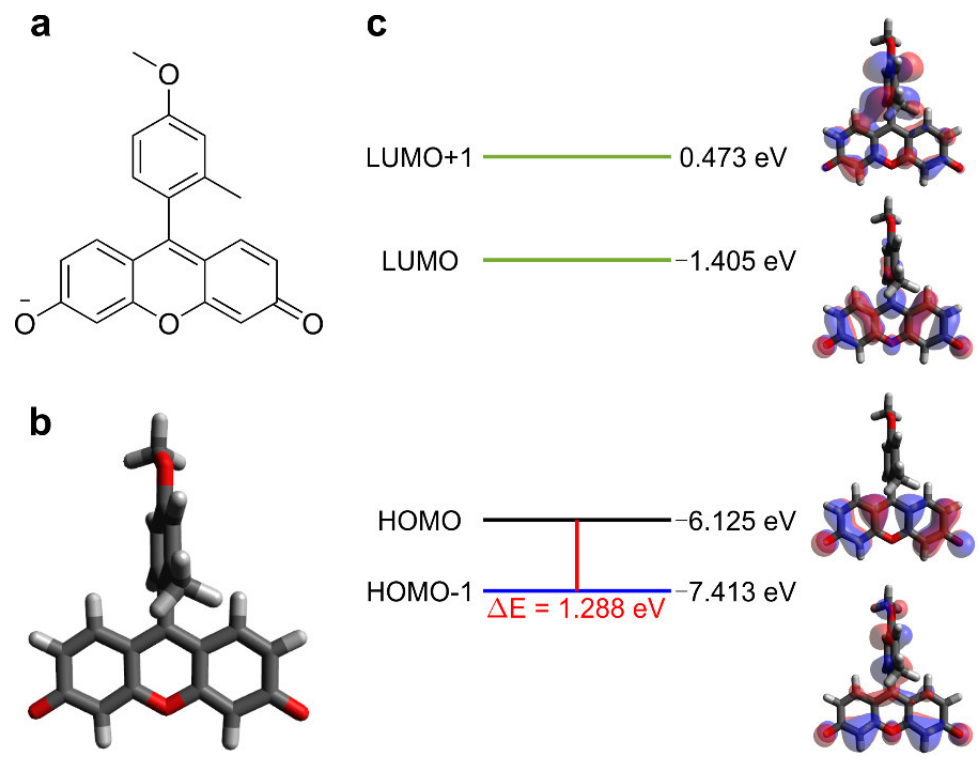

Figure S170. a) Molecular structure, b) optimized structure, c) frontier molecular orbitals and corresponding energy levels, and calculated $\Delta \mathrm{E}$ of F20 in the ground state in water at M062X/Def2SVP level. 
a<smiles>COc1ccc(C)cc1-c1c2ccc(=O)cc-2oc2cc([O-])ccc12</smiles>

C

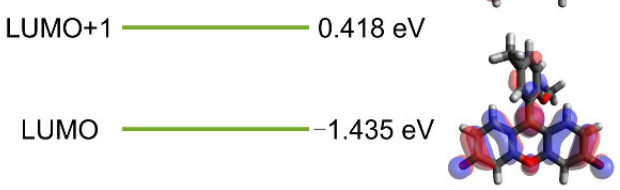

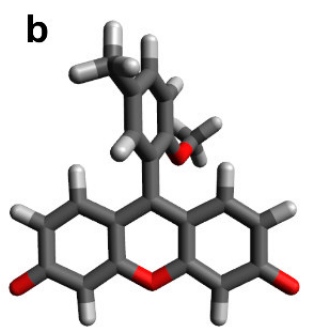

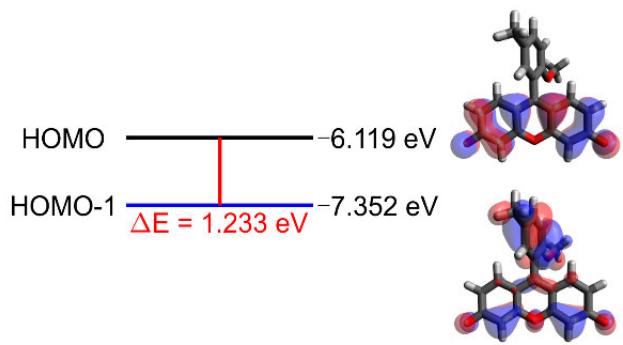

Figure S171. a) Molecular structure, b) optimized structure, c) frontier molecular orbitals and corresponding energy levels, and calculated $\Delta \mathrm{E}$ of F21 in the ground state in water at M062X/Def2SVP level.<smiles>COc1ccc(-c2c3ccc(=O)cc-3oc3cc([O-])ccc23)c(OC)c1</smiles>

\section{C}<smiles>CO[14CH3]</smiles>

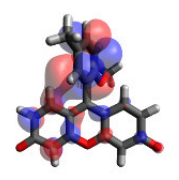

LUMO $1.429 \mathrm{eV}$
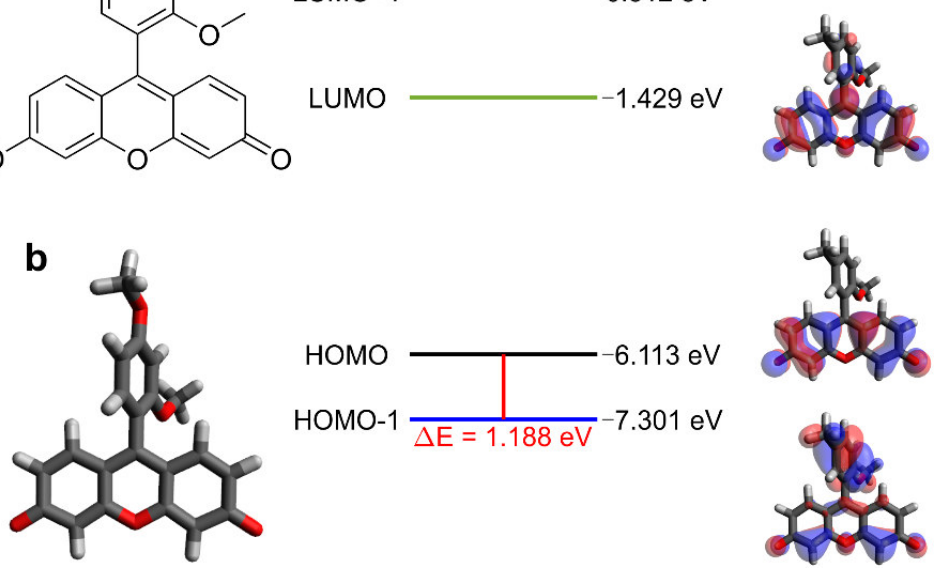

Figure S172. a) Molecular structure, b) optimized structure, c) frontier molecular orbitals and corresponding energy levels, and calculated $\Delta \mathrm{E}$ of F22 in the ground state in water at M062X/Def2SVP level. 

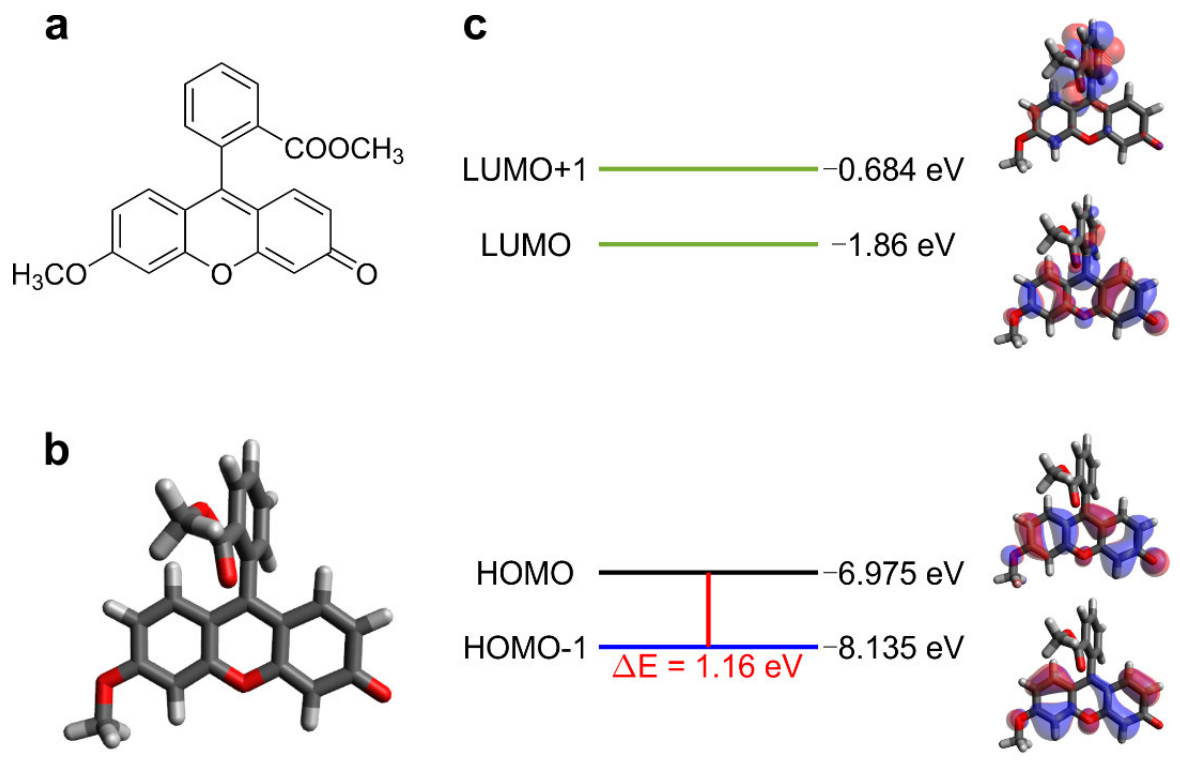

Figure S173. a) Molecular structure, b) optimized structure, c) frontier molecular orbitals and corresponding energy levels, and calculated $\triangle E$ of F23 in the ground state in PBS at M062X/Def2SVP level.
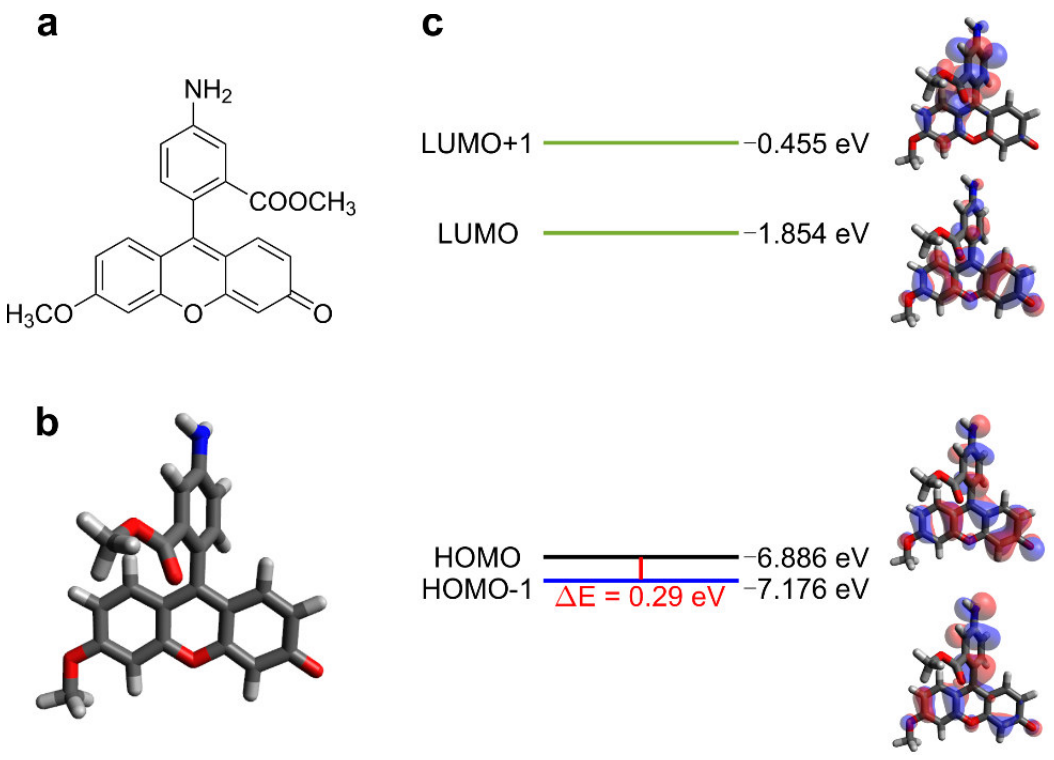

Figure S174. a) Molecular structure, b) optimized structure, c) frontier molecular orbitals and corresponding energy levels, and calculated $\triangle \mathrm{E}$ of F24 in the ground state in PBS at M062X/Def2SVP level. 


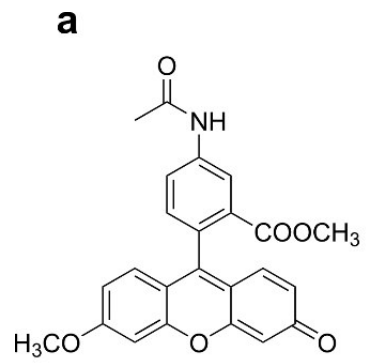

C
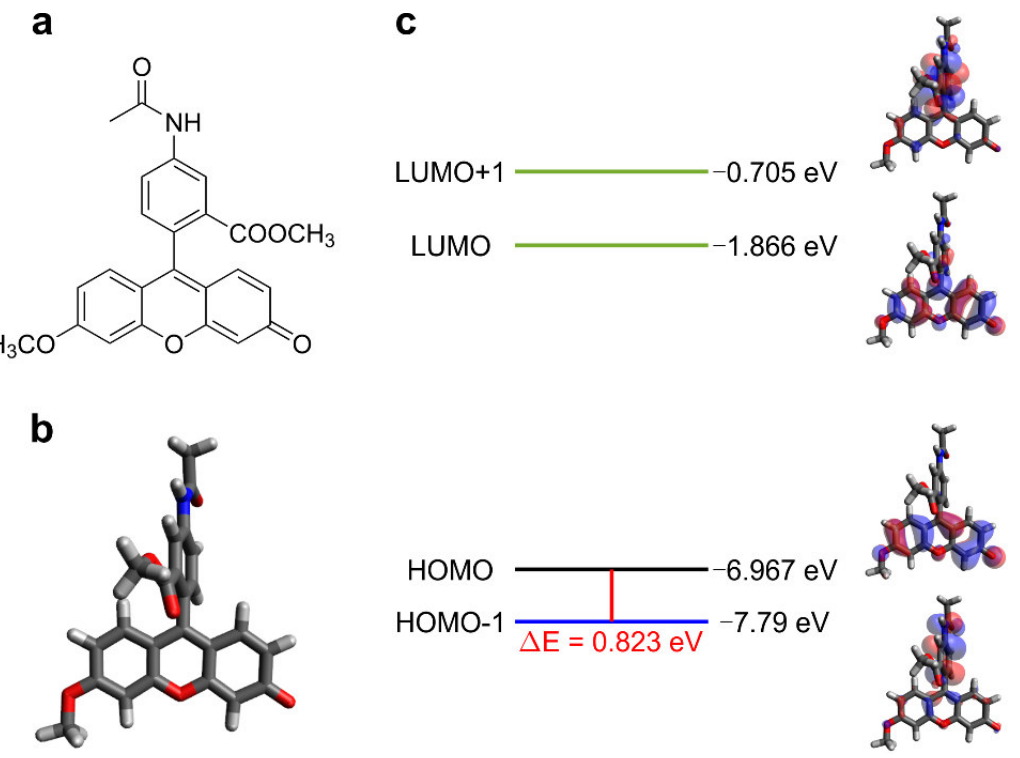

Figure S175. a) Molecular structure, b) optimized structure, c) frontier molecular orbitals and corresponding energy levels, and calculated $\Delta \mathrm{E}$ of F25 in the ground state in PBS at M062X/Def2SVP level.
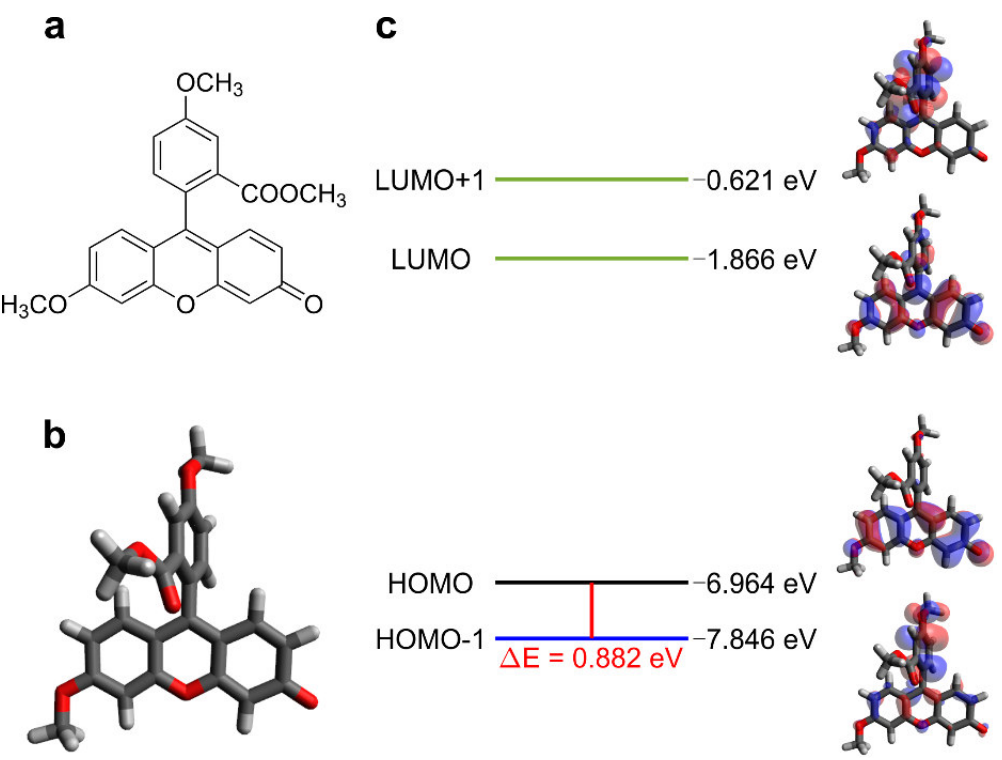

Figure S176. a) Molecular structure, b) optimized structure, c) frontier molecular orbitals and corresponding energy levels, and calculated $\triangle \mathrm{E}$ of F26 in the ground state in PBS at M062X/Def2SVP level. 
a<smiles>COc1ccc2c(-c3cc4ccccc4cc3C(C)=O)c3ccc(=O)cc-3oc2c1</smiles>

b

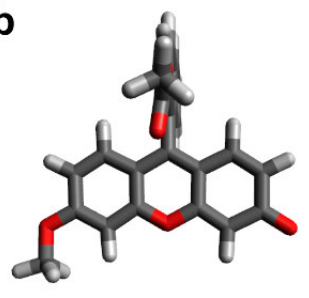

C

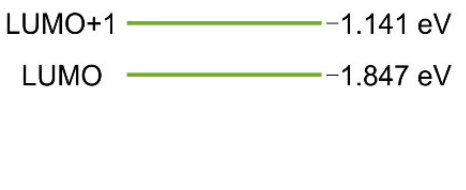

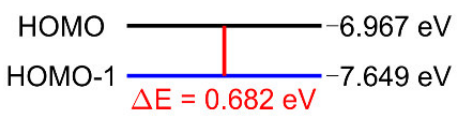

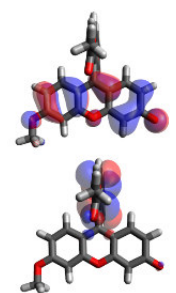

Figure S177. a) Molecular structure, b) optimized structure, c) frontier molecular orbitals and corresponding energy levels, and calculated $\triangle E$ of F27 in the ground state in PBS at M062X/Def2SVP level.

a<smiles>COC(=O)c1cc2cc3ccccc3cc2cc1-c1c2ccc(=O)cc-2oc2cc(OC)ccc12</smiles>

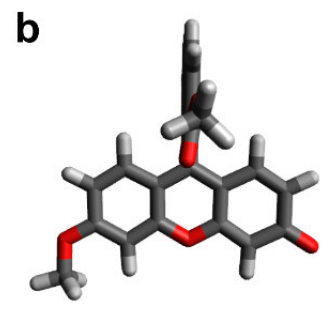

C

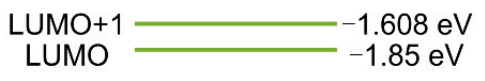

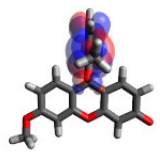

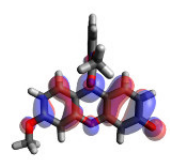

HOMO HOMO-1
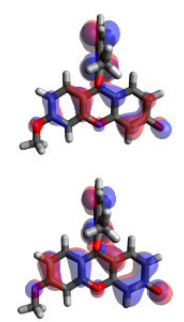

Figure S178. a) Molecular structure, b) optimized structure, c) frontier molecular orbitals and corresponding energy levels, and calculated $\triangle \mathrm{E}$ of F28 in the ground state in PBS at M062X/Def2SVP level. 

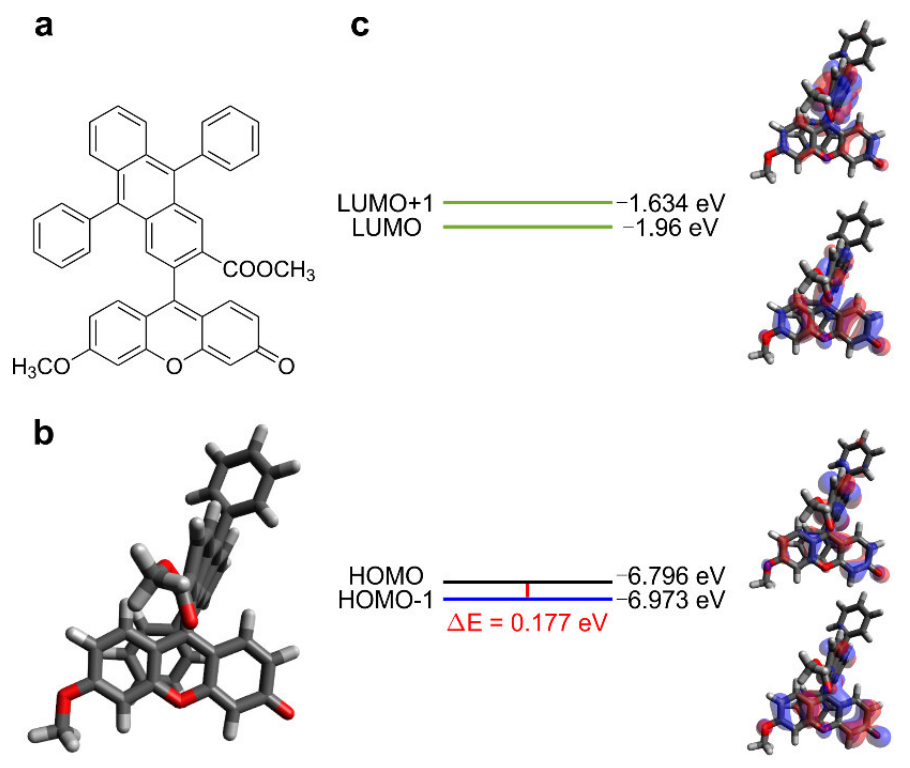

Figure S179. a) Molecular structure, b) optimized structure, c) frontier molecular orbitals and corresponding energy levels, and calculated $\triangle \mathrm{E}$ of F29 in the ground state in PBS at M062X/Def2SVP level.
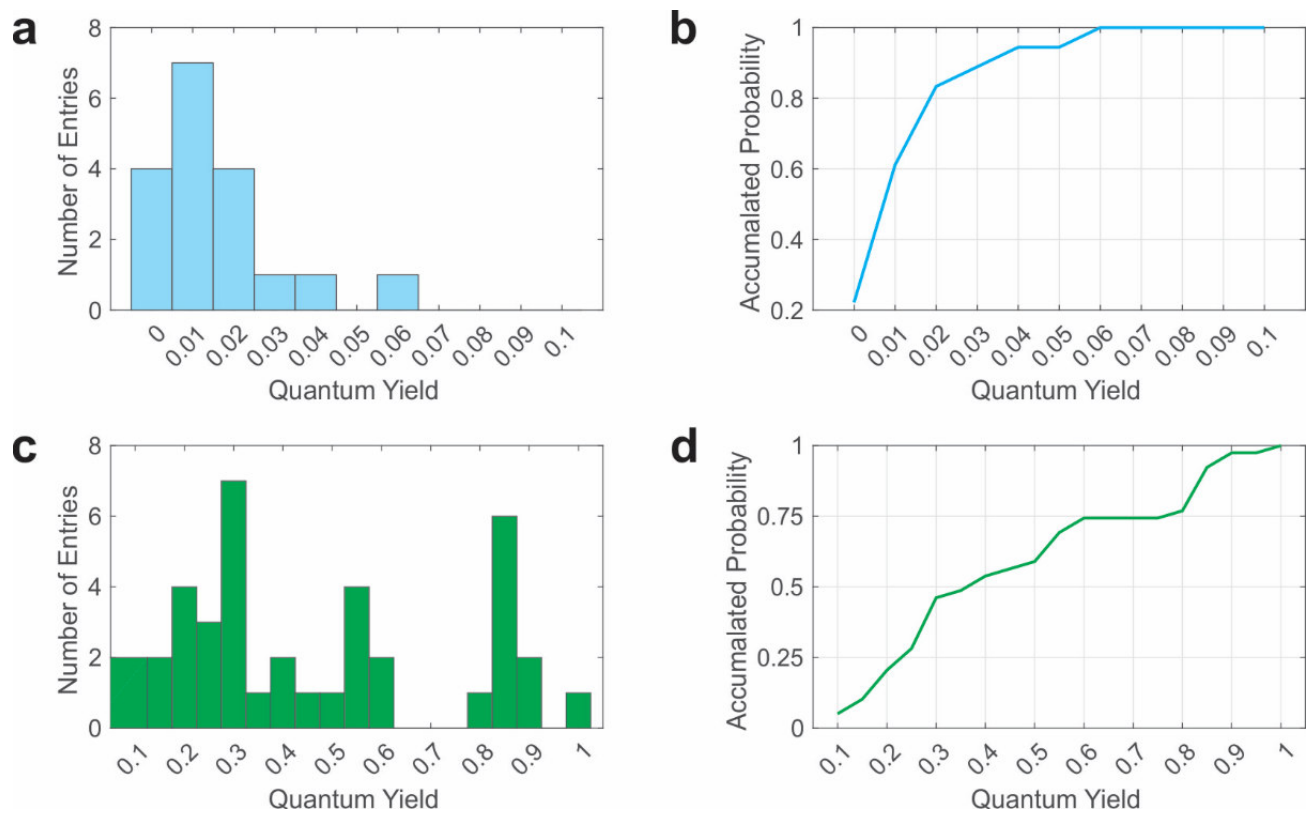

Figure S180. The histogram and accumulated probability of quantum yields for fluorescein, rhodamine and Sirhodamine derivatives in polar solvents with $(\mathrm{a}, \mathrm{b}) \Delta \mathrm{E}<0.6 \mathrm{eV}$ and $(\mathrm{c}, \mathrm{d}) \Delta \mathrm{E}>0.6 \mathrm{eV}$. 

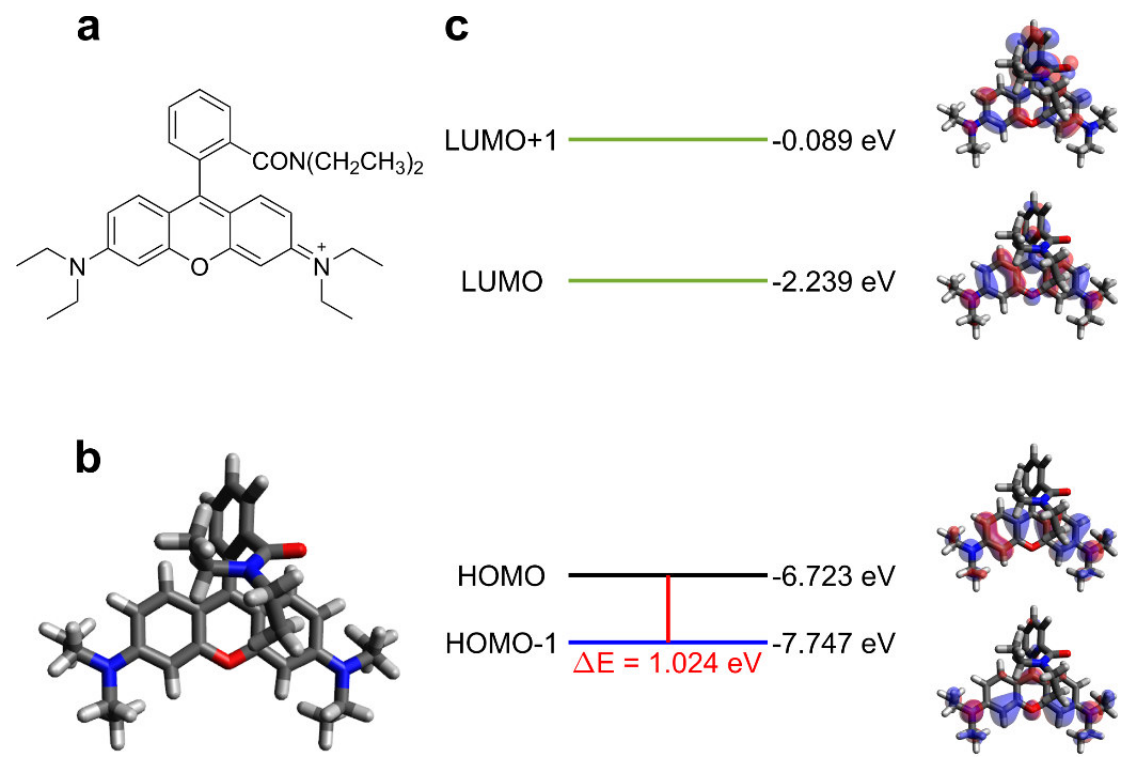

Figure S181. a) Molecular structure, b) optimized structure, c) frontier molecular orbitals and corresponding energy levels, and calculated $\Delta \mathrm{E}$ of M1 in the ground state in methanol at M062X/Def2SVP level.
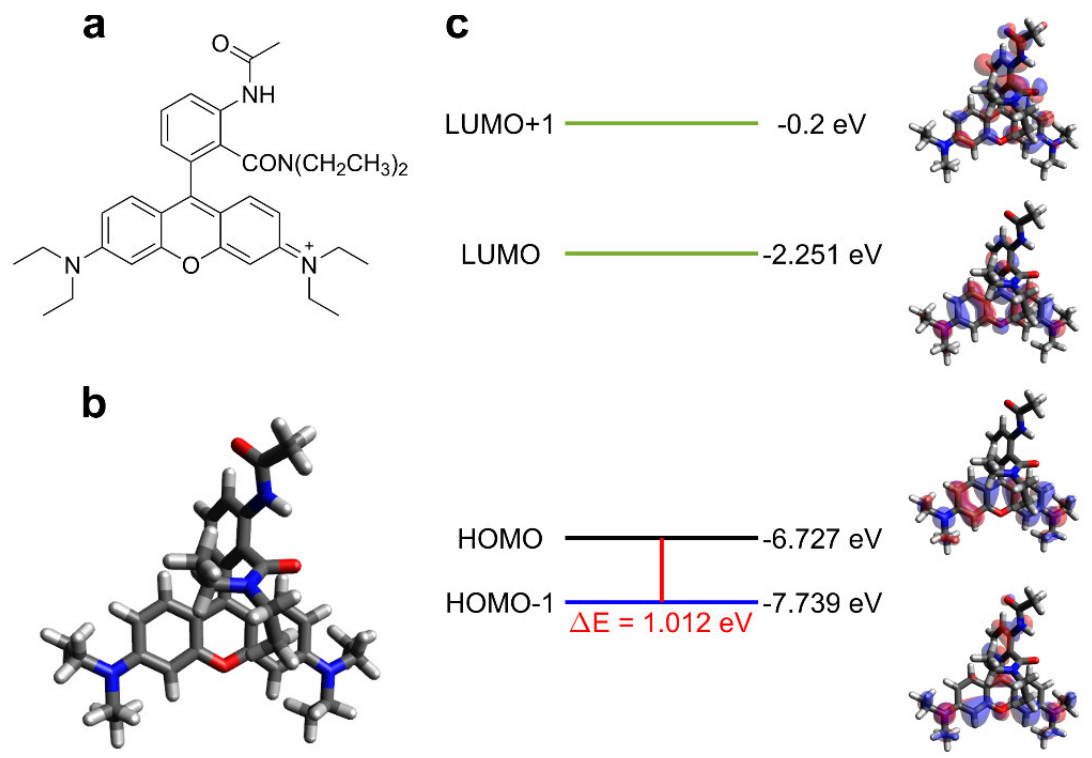

Figure S182. a) Molecular structure, b) optimized structure, c) frontier molecular orbitals and corresponding energy levels, and calculated $\Delta \mathrm{E}$ of $\mathrm{M} 2$ in the ground state in methanol at M062X/Def2SVP level. 

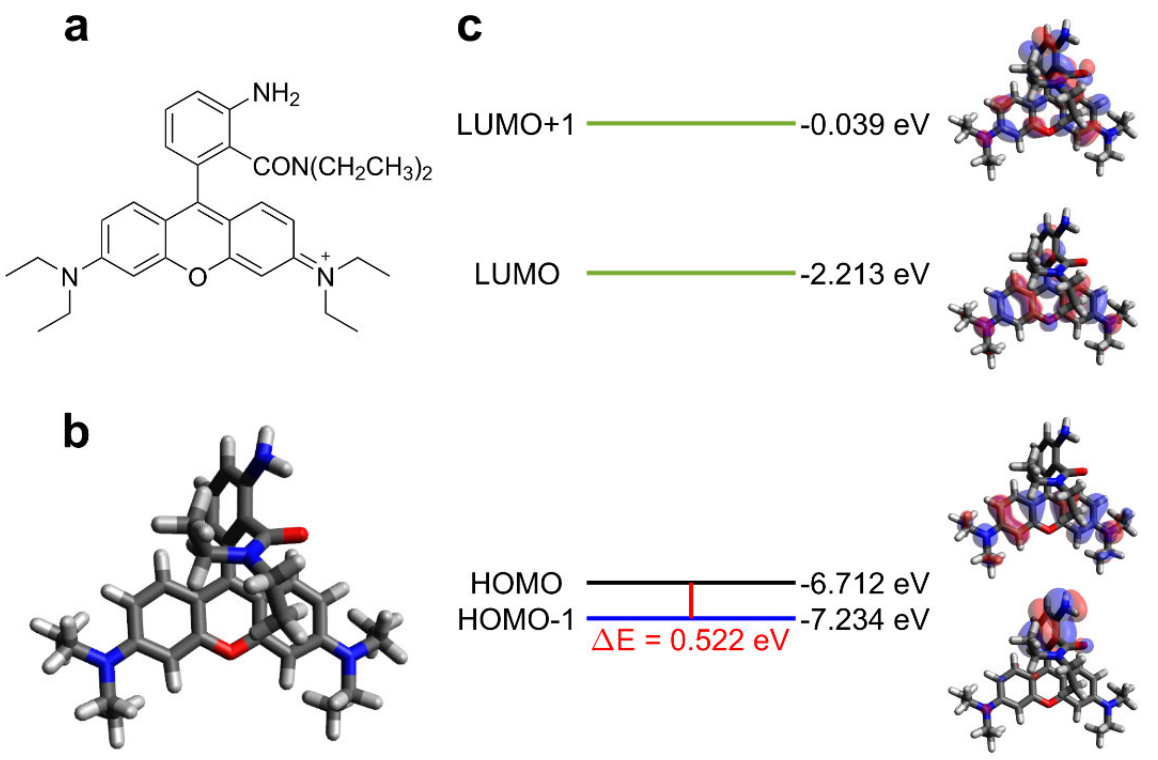

Figure S183. a) Molecular structure, b) optimized structure, c) frontier molecular orbitals and corresponding energy levels, and calculated $\Delta \mathrm{E}$ of M3 in the ground state in methanol at M062X/Def2SVP level. 
UV-Vis absorption and fluorescence spectra of M1-M3.

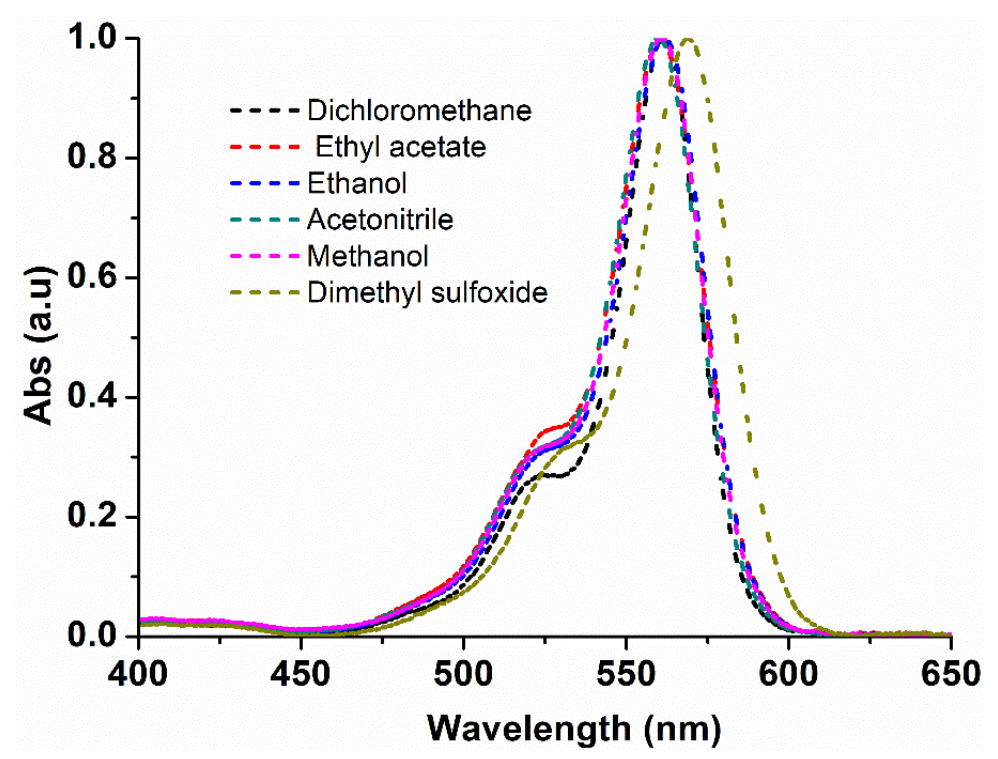

Figure S184. Normalized UV-Vis absorption spectra of M1 in dichloromethane, ethyl acetate, ethanol, acetonitrile, methanol, and dimethyl sulfoxide solutions. [M1] = $2 \mu \mathrm{M}$.

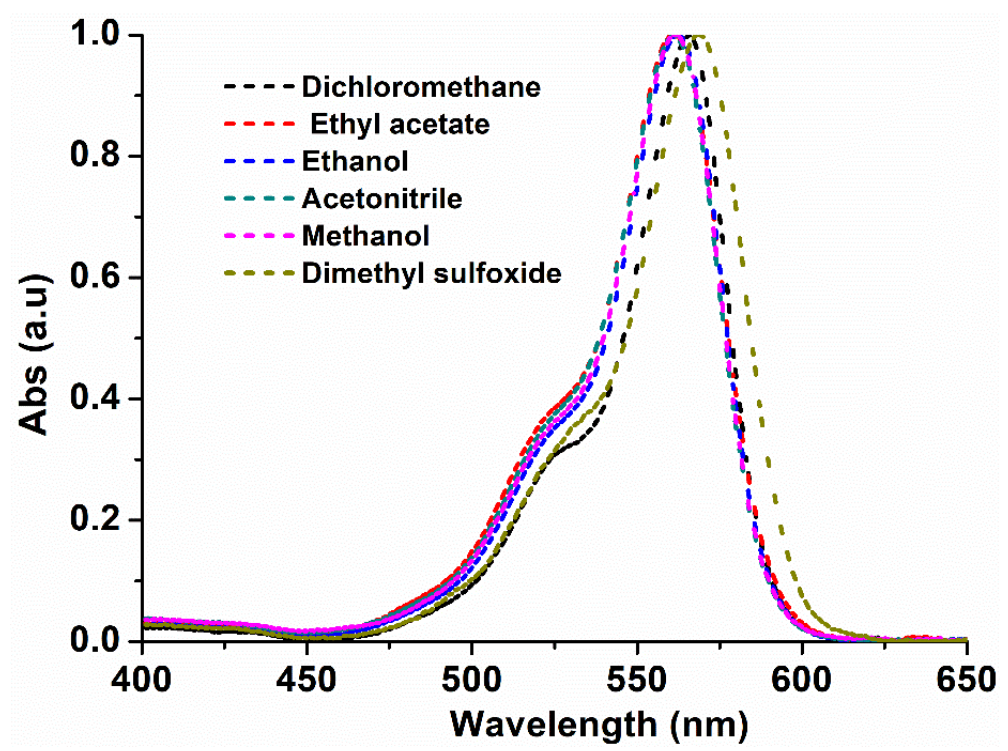

Figure S185. Normalized UV-Vis absorption spectra of M2 in dichloromethane, ethyl acetate, ethanol, acetonitrile, methanol, and dimethyl sulfoxide solutions. [M2] = $2 \mu \mathrm{M}$. 


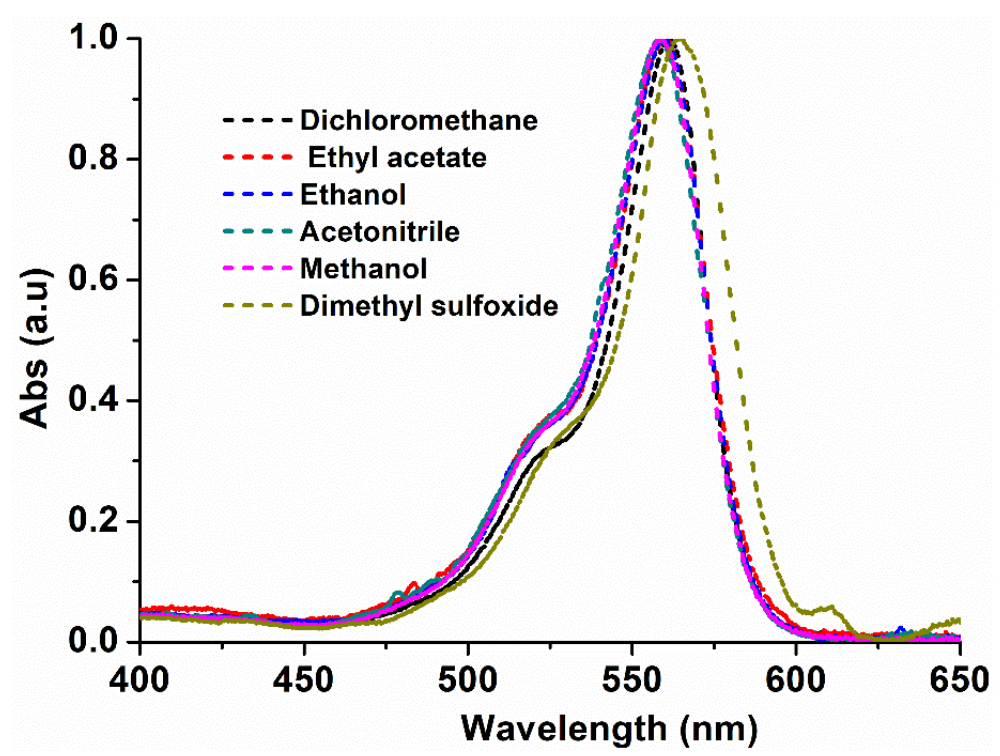

Figure S186. Normalized UV-Vis absorption spectra of M3 in dichloromethane, ethyl acetate, ethanol, acetonitrile, methanol, and dimethyl sulfoxide solutions. [M3] = $2 \mu \mathrm{M}$.

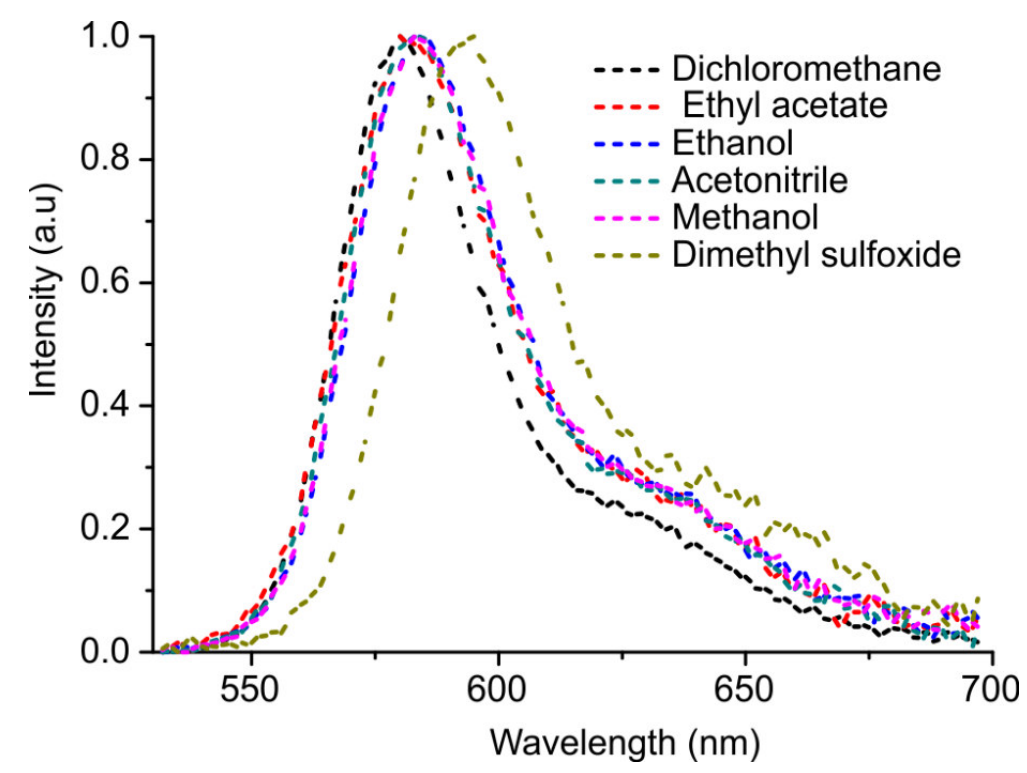

Figure S187. Normalized fluorescence spectra of M1 in dichloromethane, ethyl acetate, ethanol, acetonitrile, methanol, and dimethyl sulfoxide. [M1] = $2 \mu \mathrm{M}$; excitation wavelength $=520 \mathrm{~nm}$. 


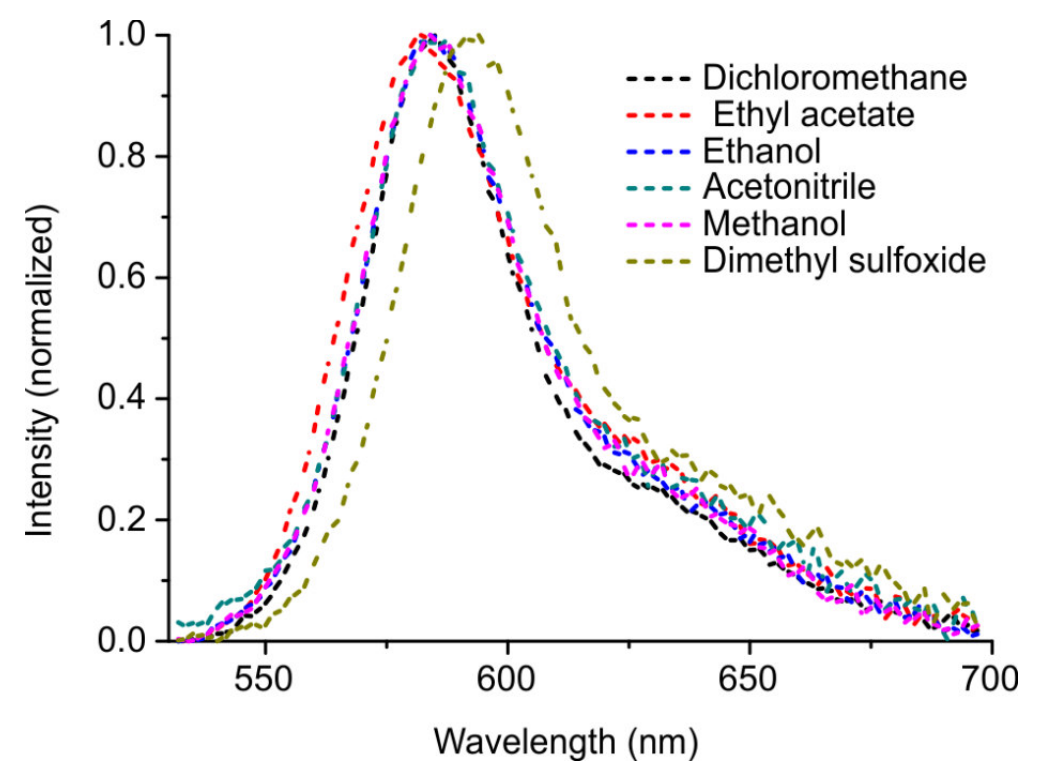

Figure S188. Normalized fluorescence spectra of M2 in dichloromethane, ethyl acetate, ethanol, acetonitrile, methanol, and dimethyl sulfoxide. [M2] = $2 \mu \mathrm{M}$; excitation wavelength $=520 \mathrm{~nm}$.

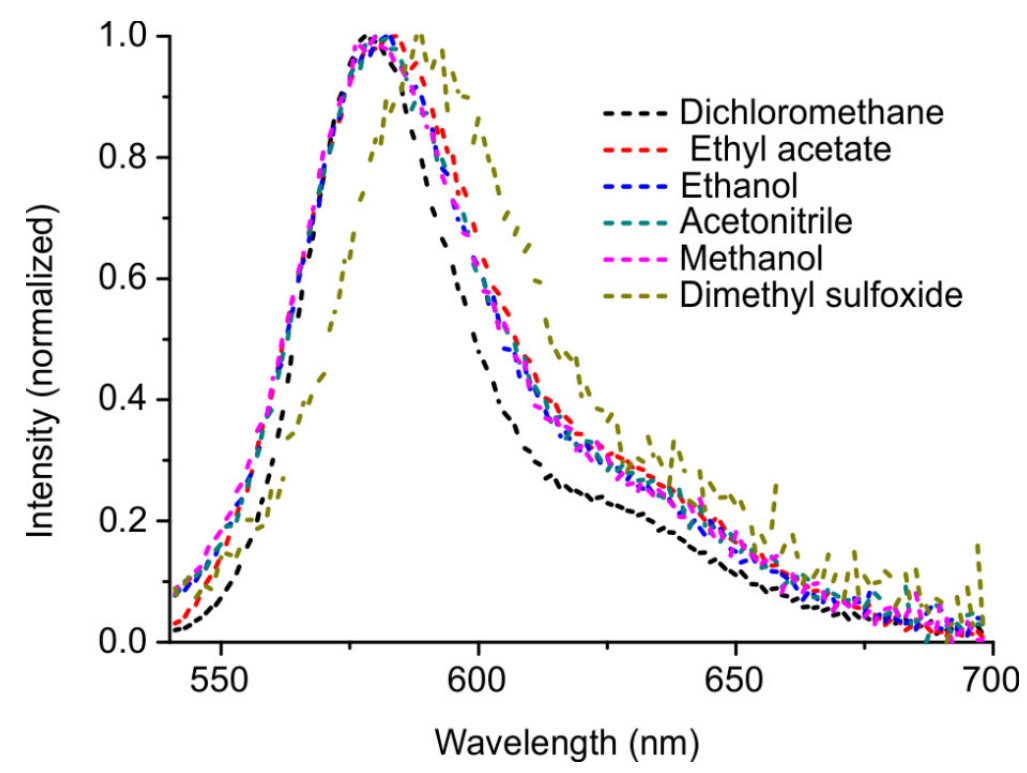

Figure S189. Normalized fluorescence spectra of M3 in dichloromethane, ethyl acetate, ethanol, acetonitrile, methanol, and dimethyl sulfoxide. [M3] = $2 \mu \mathrm{M}$; excitation wavelength $=520 \mathrm{~nm}$. 


\section{Bioimaging applications of $\mathbf{M} 2$ and $\mathbf{M} 3$}
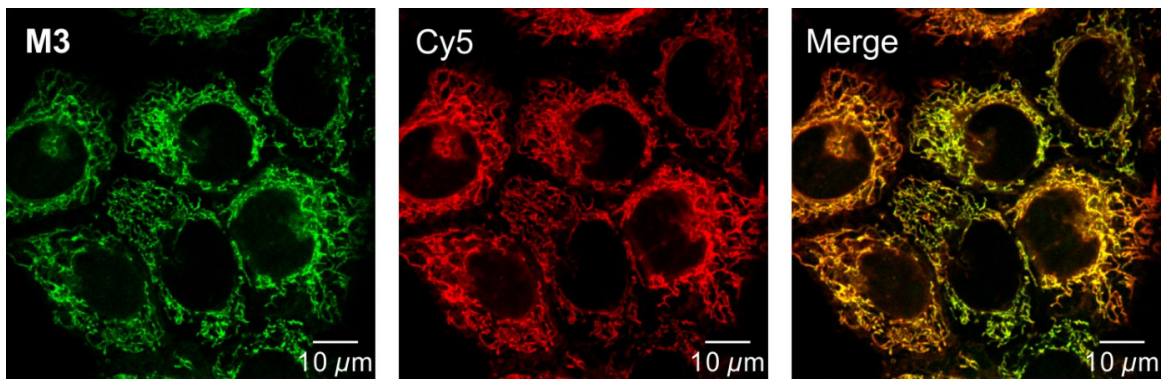

Figure S190. Co-staining of HeLa cells using M3 (3 $\mu \mathrm{M})$ and Cy5(2-((1E,3E-5E)-5-1,3,3-trimethylindolin-2ylidene)penta-1,3-dien-1-ylidene)- 1,3,3-trimethyl-3H-indol-1-ium iodide; $500 \mathrm{nM}$ ). Green channel, M3; red channel, Cy5; yellow channel, the merged image.

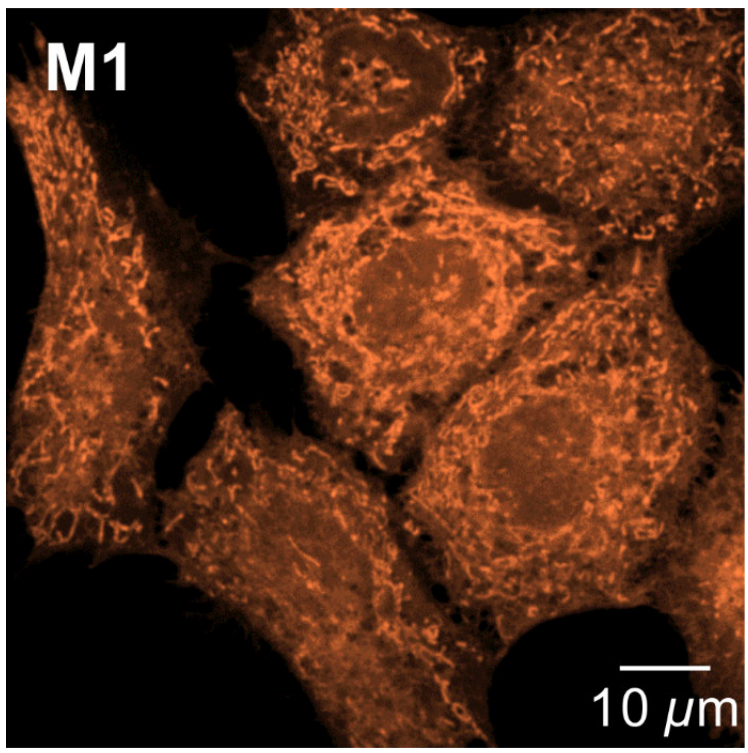

Figure S191. Stained live HeLa cell using M1 (3 $\mu \mathrm{M})$, with significant noises from cytoplasm in addition to emissions from mitochondria. 


\section{References}

1. Zhao, Y.; Truhlar, D. G., The M06 Suite of Density Functionals for Main Group Thermochemistry, Thermochemical Kinetics, Noncovalent Interactions, Excited States, and Transition Elements: Two New Functionals and Systematic Testing of Four M06-Class Functionals and 12 other Functionals. Theor. Chem. Acc. 2008, 120, 215-241.

2. Chai, J.-D.; Head-Gordon, M., Long-Range Corrected Hybrid Density Functionals with Damped Atom-Atom Dispersion Corrections. Phys. Chem. Chem. Phys. 2008, 10, 6615-6620.

3. Weigend, F.; Ahlrichs, R., Balanced Basis Sets of Split Valence, Triple Zeta Valence and Quadruple Zeta Valence Quality for H to Rn: Design and Assessment of Accuracy. Phys. Chem. Chem. Phys. 2005, 7 (18), 3297-3305.

4. Marenich, A. V.; Cramer, C. J.; Truhlar, D. G., Universal Solvation Model Based on Solute Electron Density and on a Continuum Model of the Solvent Defined by the Bulk Dielectric Constant and Atomic Surface Tensions. J. Phys. Chem. B 2009, 113, 6378-6396.

5. Caricato, M.; Mennucci, B.; Tomasi, J.; Ingrosso, F.; Cammi, R.; Corni, S.; Scalmani, G., Formation and Relaxation of Excited States in Solution: A new Time Dependent Polarizable Continuum Model Based on Time Dependent Density Functional Theory.J. Chem. Phys 2006, 124, 124520.

6. Frisch, M. J.; Trucks, G. W.; Schlegel, H. B.; Scuseria, G. E.; Robb, M. A.; Cheeseman, J. R.; Scalmani, G.; Barone, V.; Petersson, G. A.; Nakatsuji, H.; Li, X.; Caricato, M.; Marenich, A. V.; Bloino, J.; Janesko, B. G.; Gomperts, R.; Mennucci, B.; Hratchian, H. P.; Ortiz, J. V.; Izmaylov, A. F.; Sonnenberg, J. L.; Williams; Ding, F.; Lipparini, F.; Egidi, F.; Goings, J.; Peng, B.; Petrone, A.; Henderson, T.; Ranasinghe, D.; Zakrzewski, V. G.; Gao, J.; Rega, N.; Zheng, G.; Liang, W.; Hada, M.; Ehara, M.; Toyota, K.; Fukuda, R.; Hasegawa, J.; Ishida, M.; Nakajima, T.; Honda, Y.; Kitao, O.; Nakai, H.; Vreven, T.; Throssell, K.; Montgomery Jr., J. A.; Peralta, J. E.; Ogliaro, F.; Bearpark, M. J.; Heyd, J. J.; Brothers, E. N.; Kudin, K. N.; Staroverov, V. N.; Keith, T. A.; Kobayashi, R.; Normand, J.; Raghavachari, K.; Rendell, A. P.; Burant, J. C.; Iyengar, S. S.; Tomasi, J.; Cossi, M.; Millam, J. M.; Klene, M.; Adamo, C.; Cammi, R.; Ochterski, J. W.; Martin, R. L.; Morokuma, K.; Farkas, O.; Foresman, J. B.; Fox, D. J. Gaussian 16 Rev. C.01, Wallingford, CT, 2016. 7. Aydın Tekdaş, D.; Viswanathan, G.; Zehra Topal, S.; Looi, C. Y.; Wong, W. F.; Min Yi Tan, G.; Zorlu, Y.; Gürek, A. G.; Lee, H. B.; Dumoulin, F., Antimicrobial Activity of a Quaternized BODIPY Against Staphylococcus Strains. Org. Biomol. Chem. 2016, 14, 2665-2670.

8. Ye, Z.; Xiong, C.; Pan, J.; Su, D.; Zeng, L., Highly Photostable, Lysosome-Targeted BODIPYs with Green to Near-Infrared Emission for Lysosome Imaging in Living Cells. Dyes Pigments 2018, 155, 30-35.

9. Ramos-Torres, Á.; Avellanal-Zaballa, E.; Prieto-Castañeda, A.; García-Garrido, F.; Bañuelos, J.; Agarrabeitia, A. R.; Ortiz, M. J., FormyIBODIPYs by PCC-Promoted Selective Oxidation of $\alpha-$ MethyIBODIPYs. Synthetic Versatility and Applications. Org. Lett. 2019, 21, 4563-4566.

10. Quan, L.; Lin, W.; Sun, T.; Xie, Z.; Huang, Y.; Jing, X., Green Photocatalysis with Oxygen Sensitive BODIPYs under Visible Light. Catal. Lett. 2014, 144, 308-313.

11. Mula, S.; Ulrich, G.; Ziessel, R., Dual Bodipy Fluorophores Linked by Polyethyleneglycol Spacers. Tetrahedron Lett. 2009, 50, 6383-6388.

12. Ying, L.-Q.; Branchaud, B. P., Selective Labeling and Monitoring $\mathrm{pH}$ Changes of Lysosomes in Living Cells with Fluorogenic pH Sensors. Bioorg. Med. Chem. Lett. 2011, 21, 3546-3549. 
13. Urano, Y.; Asanuma, D.; Hama, Y.; Koyama, Y.; Barrett, T.; Kamiya, M.; Nagano, T.; Watanabe, T.; Hasegawa, A.; Choyke, P. L.; Kobayashi, H., Selective Molecular Imaging of Viable Cancer Cells with pHActivatable Fluorescence Probes. Nat. Med. 2008, 15, 104.

14. Kim, T.-I.; Park, J.; Park, S.; Choi, Y.; Kim, Y., Visualization of Tyrosinase Activity in Melanoma Cells by a BODIPY-Based Fluorescent Probe. Chem. Commun. 2011, 47, 12640-12642.

15. Hu, W.; Zhang, X.-F.; Lu, X.; Lan, S.; Tian, D.; Li, T.; Wang, L.; Zhao, S.; Feng, M.; Zhang, J., Modifying the Meso-Phenyl with Electron Donating Amino Groups Strongly Enhances BODIPY's Ability as Good Singlet Oxygen Photosensitizer. Dyes Pigments 2018, 149, 306-314.

16. Sunahara, H.; Urano, Y.; Kojima, H.; Nagano, T., Design and Synthesis of a Library of BODIPYBased Environmental Polarity Sensors Utilizing Photoinduced Electron-Transfer-Controlled Fluorescence ON/OFF Switching. J. Am. Chem. Soc. 2007, 129, 5597-5604.

17. Ueno, T.; Urano, Y.; Kojima, H.; Nagano, T., Mechanism-Based Molecular Design of Highly Selective Fluorescence Probes for Nitrative Stress. J. Am. Chem. Soc. 2006, 128, 10640-10641.

18. Baruah, M.; Qin, W.; Basarić, N.; De Borggraeve, W. M.; Boens, N., BODIPY-Based Hydroxyaryl Derivatives as Fluorescent pH Probes. J. Org. Chem. 2005, 70, 4152-4157.

19. Lincoln, R.; Greene, L. E.; Krumova, K.; Ding, Z.; Cosa, G., Electronic Excited State Redox Properties for BODIPY Dyes Predicted from Hammett Constants: Estimating the Driving Force of Photoinduced Electron Transfer. J. Phys. Chem. A 2014, 118, 10622-10630.

20. Hu, W.; Liu, M.; Zhang, X.-F.; Wang, Y.; Wang, Y.; Lan, H.; Zhao, H., Can BODIPY-Electron Acceptor Conjugates Act As Heavy Atom-Free Excited Triplet State and Singlet Oxygen Photosensitizers via Photoinduced Charge Separation-Charge Recombination Mechanism? J. Phys. Chem. C 2019, 123, 1594415955.

21. Prasannan, D.; Arunkumar, C., A "Turn-On-and-Off” pH Sensitive BODIPY Fluorescent Probe for Imaging E. Coli Cells. New J. Chem. 2018, 42, 3473-3482.

22. Matsumoto, T.; Urano, Y.; Shoda, T.; Kojima, H.; Nagano, T., A Thiol-Reactive Fluorescence Probe Based on Donor-Excited Photoinduced Electron Transfer: Key Role of Ortho Substitution. Org. Lett. 2007, 9, 3375-3377.

23. Zhang, H.-X.; Chen, J.-B.; Guo, X.-F.; Wang, H.; Zhang, H.-S., Highly Sensitive Low-Background Fluorescent Probes for Imaging of Nitric Oxide in Cells and Tissues. Anal. Chem. 2014, 86, 3115-3123.

24. Zhang, J.; Ji, X.; Ren, H.; Zhou, J.; Chen, Z.; Dong, X.; Zhao, W., Meso-Heteroaryl BODIPY Dyes as Dual-Responsive Fluorescent Probes for Discrimination of Cys from Hcy and GSH. Sens. Actuators, B 2018, 260, 861-869.

25. Gabe, Y.; Urano, Y.; Kikuchi, K.; Kojima, H.; Nagano, T., Highly Sensitive Fluorescence Probes for Nitric Oxide Based on Boron Dipyrromethene ChromophoreRational Design of Potentially Useful Bioimaging Fluorescence Probe. J. Am. Chem. Soc. 2004, 126, 3357-3367.

26. Chen, Y.; Wan, L.; Zhang, D.; Bian, Y.; Jiang, J., Modulation of the Spectroscopic Property of BODIPY Derivates through Tuning the Molecular Configuration. Photochem. Photobiol. Sci. 2011, 10, 10301038.

27. Qi, Q.; Chi, W.; Li, Y.; Qiao, Q.; Chen, J.; Miao, L.; Zhang, Y.; Li, J.; Ji, W.; Xu, T.; Liu, X.; Yoon, J.; Xu, Z., A H-bond Strategy to Develop Acid-Resistant Photoswitchable Rhodamine Spirolactams for SuperResolution Single-Molecule Localization Microscopy. Chem. Sci. 2019, 10, 4914-4922. 
28. Umezawa, K.; Yoshida, M.; Kamiya, M.; Yamasoba, T.; Urano, Y., Rational Design of Reversible Fluorescent Probes for Live-Cell Imaging and Quantification of Fast Glutathione Dynamics. Nat. Chem. 2016, 9, 279.

29. Zhang, X.-F.; Su, N.; Lu, X.; Jia, W., Benzoate-Modified Rhodamine Dyes: Large Change in Fluorescence Properties Due to Photoinduced Electron Transfer. J. Lumin. 2016, 179, 511-517.

30. Shen, S.; Yu, J.; Lu, Y.; Zhang, S.; Yi, X.; Gao, B., Near-Infrared Probes Based on Fluorinated Sirhodamine for Live Cell Imaging. RSC Adv. 2017, 7, 10922-10927.

31. Beija, M.; Afonso, C. A. M.; Martinho, J. M. G., Synthesis and applications of Rhodamine derivatives as fluorescent probes. Chem. Soc. Rev. 2009, 38, 2410-2433.

32. Abo, M.; Urano, Y.; Hanaoka, K.; Terai, T.; Komatsu, T.; Nagano, T., Development of a Highly Sensitive Fluorescence Probe for Hydrogen Peroxide. J. Am. Chem. Soc. 2011, 133, 10629-10637.

33. Urano, Y.; Kamiya, M.; Kanda, K.; Ueno, T.; Hirose, K.; Nagano, T., Evolution of Fluorescein as a Platform for Finely Tunable Fluorescence Probes. J. Am. Chem. Soc. 2005, 127, 4888-4894.

34. Miura, T.; Urano, Y.; Tanaka, K.; Nagano, T.; Ohkubo, K.; Fukuzumi, S., Rational Design Principle for Modulating Fluorescence Properties of Fluorescein-Based Probes by Photoinduced Electron Transfer. J. Am. Chem. Soc. 2003, 125, 8666-8671.

35. Kuss-Petermann, M.; Wenger, O. S., Increasing Electron-Transfer Rates with Increasing DonorAcceptor Distance. Angew. Chem. Int. Ed. 2016, 55, 815-819.

36. Alkindi, A. S., Al-Wahaibi, Y. M., Muggeridge. A. H., Physical Properties (Density, Excess Molar Volume, Viscosity, Surface Tension, and Refractive Index) of Ethanol + Glycerol. J. Chem. Eng. Data 2008, 53, 2793-2796. 